\author{
ZENTRUM \\ FÜR BIODIVERSITÄT UND NACHHALTIGE LANDNUTZUNG \\ SEKTION \\ BIOdIVERSITÄT, ÖKOLOgIE UND NATURSCHUTZ \\ - Centre of Biodiversity and Sustainable Land USe - \\ SECTION: BIOdIVERSITY, ECOLOgy AND NATURE CONSERVATION
}

\title{
Effects of forest structure and dynamics on vascular epiphyte assemblages - Functional trait analyses and modelling studies
}

\author{
Dissertation zur Erlangung des Doktorgrades der \\ Mathematisch-Naturwissenschaftlichen Fakultäten der \\ Georg-August-Universität Göttingen
}

\author{
vorgelegt von \\ Dipl.-Geoökol. \\ Gunnar Petter \\ aus \\ Heide (Holstein)
}

Göttingen, April, 2016 
Referentin/Referent: Prof. Dr. Holger Kreft

Korreferentin/Korreferent: Prof. Dr. Gerhard Zotz

Tag der Disputation: 2. Mai 2016 



\section{Table of contents}

LIST OF TABLES

LIST OF FIGURES

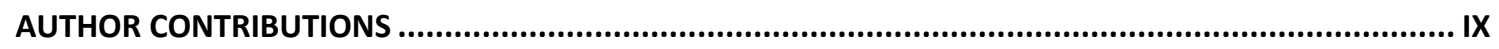

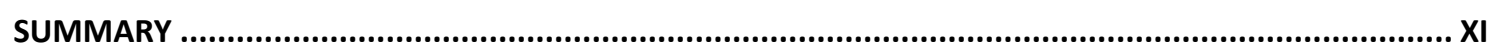

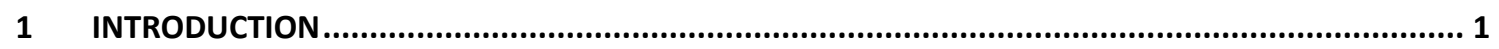

2 FUNCTIONAL LEAF TRAITS OF VASCULAR EPIPHYTES: VERTICAL TRENDS WITHIN THE FOREST, INTRA- AND INTERSPECIFIC TRAIT VARIABILITY, AND TAXONOMIC SIGNALS ................................... 7

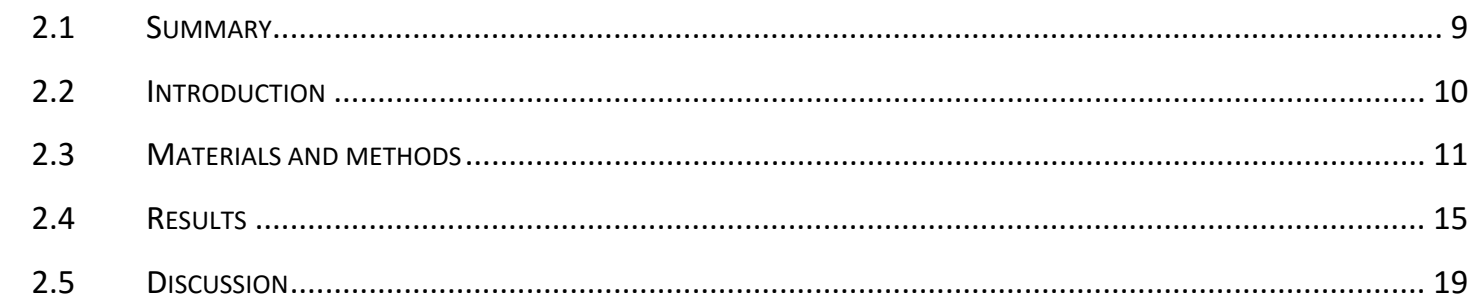

3 BRANCHFALL AS A DEMOGRAPHIC FILTER FOR EPIPHYTE COMMUNITIES: LESSONS FROM FOREST

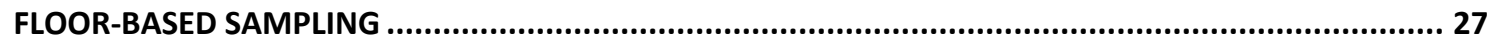

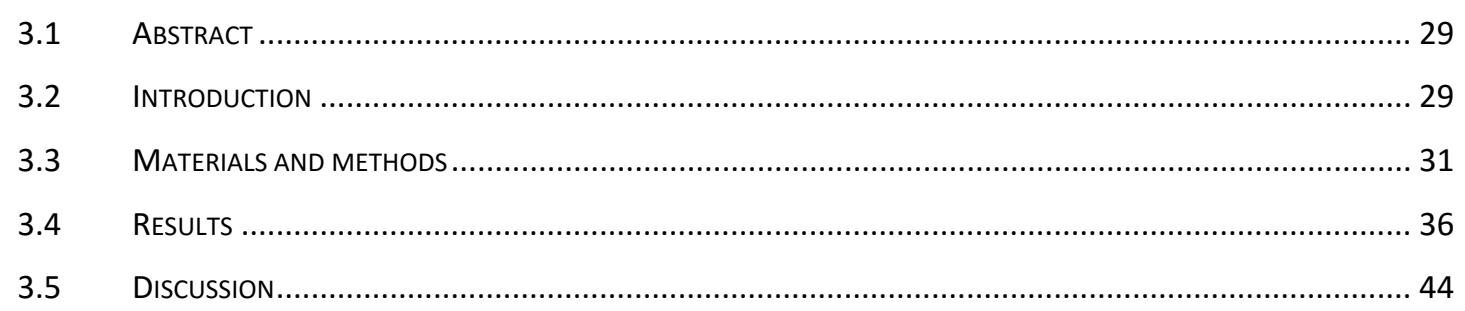

4 MODELLING THE LONG-TERM DYNAMICS OF TROPICAL FORESTS USING FUNCTIONALSTRUCTURAL TREE MODELS: LEAF TRAIT TRADE-OFFS AS PREDICTOR OF WHOLE-TREE LIFE HISTORY

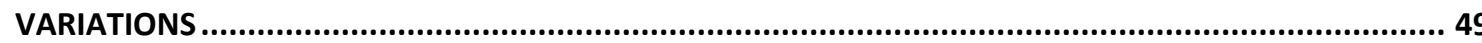

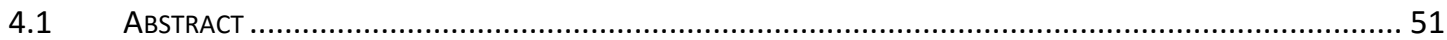

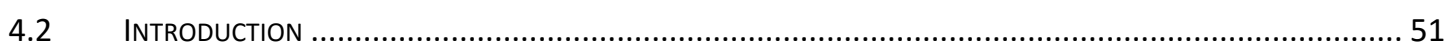

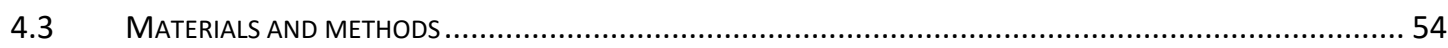

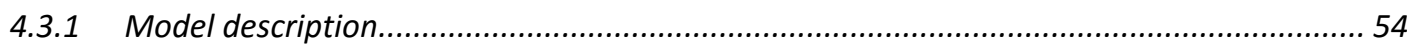

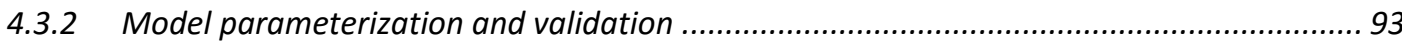

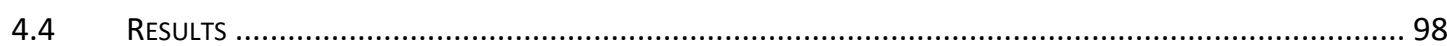

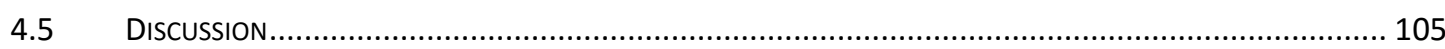


5 SIMULATING THE INFLUENCE OF FOREST DYNAMICS ON STRUCTURE AND DYNAMICS OF EPIPHYTE ASSEMBLAGES BASED ON A COUPLED FOREST-EPIPHYTE MODEL..................................115

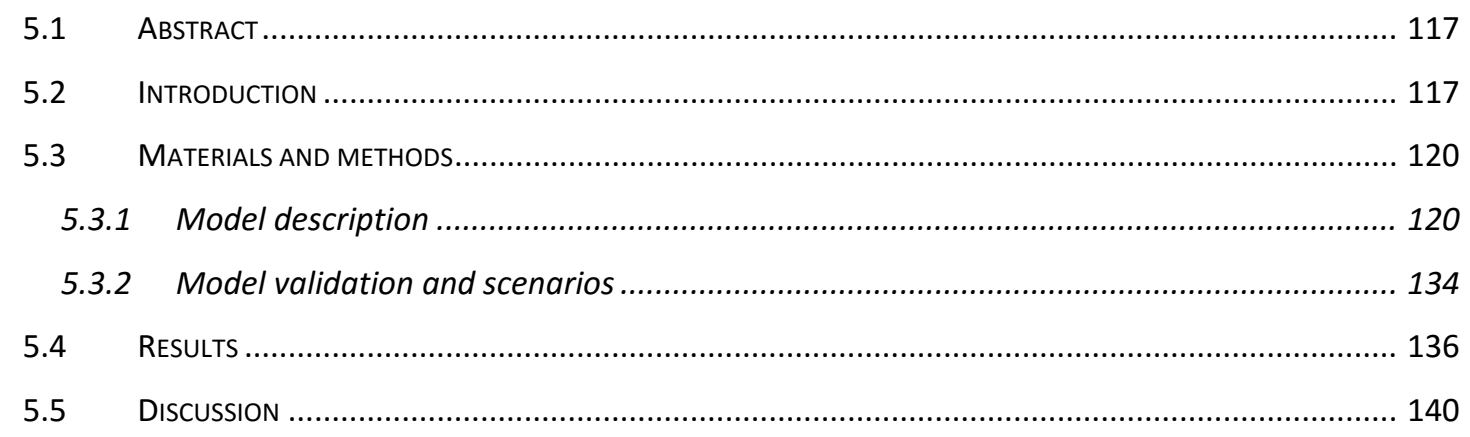




\section{List of tables}

TABle 2.1. MEAN LEAF TRAIT VALUES \pm SD OF THE MAJOR TAXONOMIC GROUPS OF VASCULAR EPIPHYTES (AROIDS, BROMELIADS, ORCHIDS, FERNS) IN A PANAMANIAN LOWLAND FOREST................................................... 18

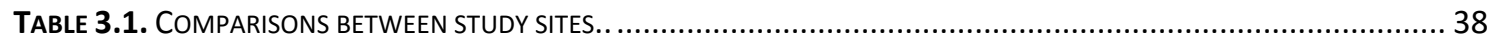

TABLE 4.1. STATE VARIABLES, FUNCTIONAL AND STRUCTURAL TRAITS OF THE FSFM............................................. 57

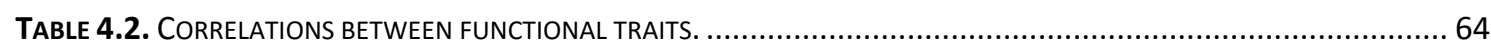

TABLE 4.3. RANGES OF FOREST ATTRIBUTES IN NEOTROPICAL LOWLAND FORESTS. ............................................... 97

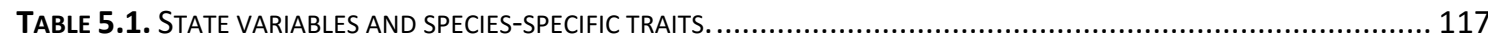

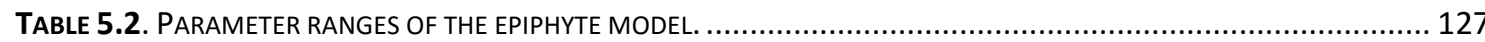

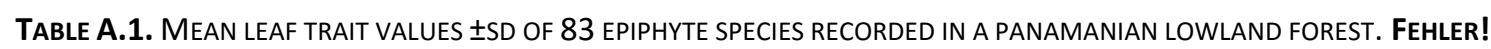
TEXTMARKE NICHT DEFINIERT.

TABle A.2. AIC-BASED COMPARISONS OF Generalized LINEAR MIXED MODELS 193

TABLE A.3. SUMMARY STATISTICS OF LINEAR MODELS TESTING TRAIT-HEIGHT RELATIONSHIPS OF VASCULAR EPIPHYTES FOR 10 LEAF TRAITS

TABLE A.4. SUMMARY STATISTICS OF LINEAR MODELS TESTING TRAIT-HEIGHT RELATIONSHIPS OF VASCULAR EPIPHYTES FOR 6 LEAF TRAITS 195

TABle A.5. PAIRWISE CORRELATIONS BETWEen LEAF TRAITS OF VASCULAR EPIPHYTES. 195

TABLE A.6. AIC-BASED COMPARISONS OF LINEAR MOdELS ANALYZING THE RELATIONSHIP BETWEEN LEAF TRAITS AND HEIGHT ABOVE GROUND. 196

TABle A.7. PROPORTION OF SPECIES WITH SIGNIFICANT TRAIT-HEIGHT RELATIONSHIPS FOR SIX LEAF TRAITS ..... 196

TABLE B.1. LIST OF VASCULAR HOLOEPIPHYTES FOUND ON THE FOREST FLOOR PER STUDY SITE. 197

TABLE B.2. LIST OF VASCULAR HOLOEPIPHYTES FOUND IN THE CANOPY PER FOREST. ........................................... 198

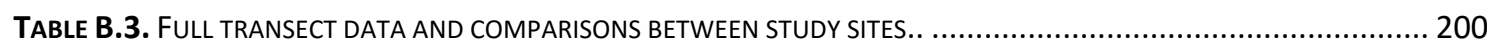

TABLE B.4. GENERALIZED ADDITIVE MIXED-EFFECTS MODELS INVESTIGATING THE INFLUENCE OF BRANCH DIAMETER ON DIFFERENT VARIABLES 201

TABLE B.5. INDICATOR SPECIES FOR THE COMPOSITIONAL DIFFERENCE BETWEEN GROUND AND CANOPY .......................201

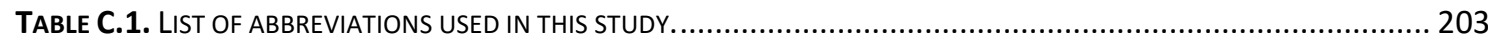

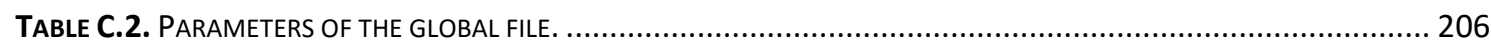

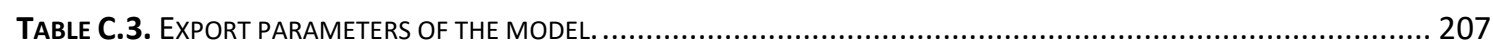

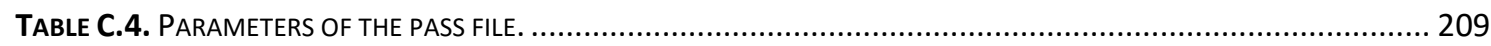

TABle C.5. ForeSt ATtRIBUtES IN NEOTROPICAL FORESTS BASED ON A LITERATURE REVIEW................................. 211

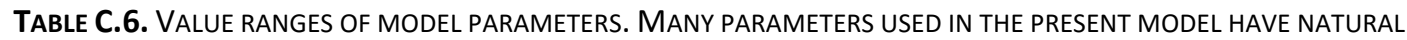
RANGES, WHICH WERE ESTIMATED BASED ON LITERATURE VALUES.

TABLE C.7. RESULTS OF SENSITIVITY ANALYSIS, IN WHICH THE EFFECT OF CHANGES IN IMPORTANT MODEL PARAMETERS ON THE MAIN FOREST ATTRIBUTES WERE RECORDED 215

TABLE D.1. SiMULATED MORTALITY RATES 


\section{List of figures}

FIGURE 2.1. TRAIT-HEIGHT RELATIONSHIPS OF VASCULAR EPIPHYTES FOR TEN LEAF TRAITS

FIGURE 2.2. DISPERSION OF TRAIT SYNDROMES OF EPIPHYTE SPECIES IN THE PCA TRAIT SPACE BASED ON SIX LEAF TRAITS OF

1,151 INDIVIDUALS. 19

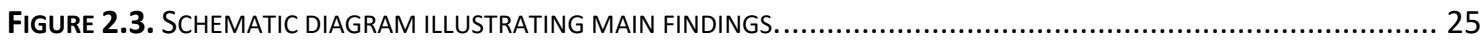

FIGURE 3.1. SCHEMATIC FIGURE SUMMARIZING THE EXPECTED TRENDS WITH INCREASING BRANCH DIAMETER .................. 31

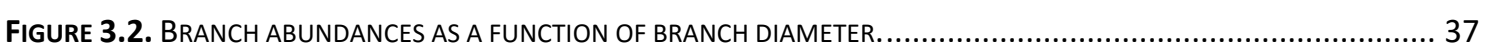

FigURE 3.3. SPECIES ACCUMULATION CURVES BASED ON FOREST FLOOR-BASED SAMPLING OF EPIPHYTES........................... 39

Figure 3.4. ABSOLUTE EPIPHYTE ABUNDANCE AND RICHNESS PER TRANSECT AS A FUNCTION OF BRANCH DIAMETER........40

FIGURE 3.5. EPIPHYTE ABUNDANCE AND RICHNESS PER BRANCH AS A FUNCTION OF BRANCH DIAMETER........................41

FIGURE 3.6. PROPORTION OF ADULTS AS A FUNCTION OF BRANCH DIAMETER ............................................... 41

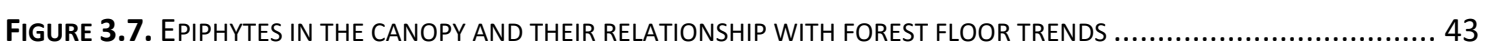

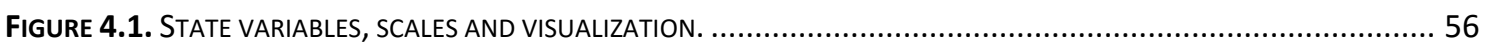

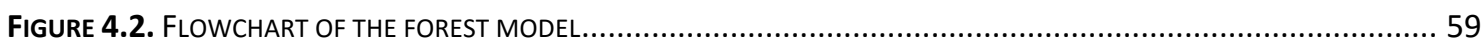

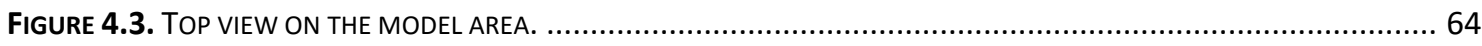

FIGURE 4.4. ILLUSTRATION OF VOXELS CONSIDERED IN CALCULATION OF EFFECTIVE LIGHT INTENSITY.............................66

FIGURE 4.5. ILLUSTRATION OF PRINCIPLES APPLIED WHEN A SMALL FOREST FRAGMENT OR A FOREST STAND WITHIN A LARGER

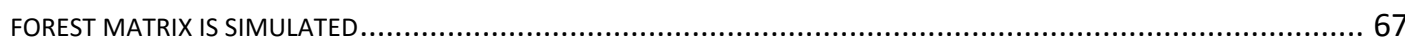

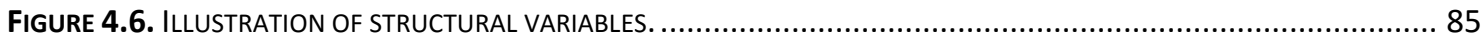

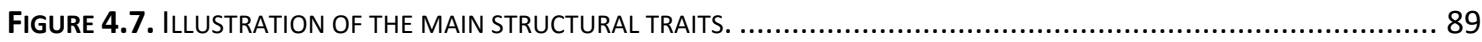

FIGURE 4.8. EXPORTABLE MODEL RESULTS AT THE THREE HIERARCHICAL SCALES: (A) TREE COMPONENTS, (B) INDIVIDUAL

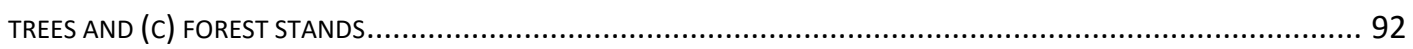

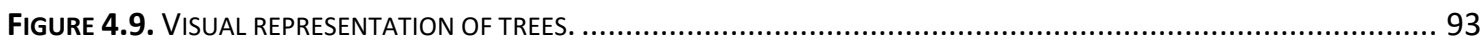

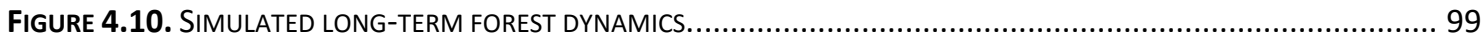

Figure 4.11. FreQuency distributions: (A) TREe DBH, (B) tREE HEIGHT, AND (C) TREe AGE.............................. 100

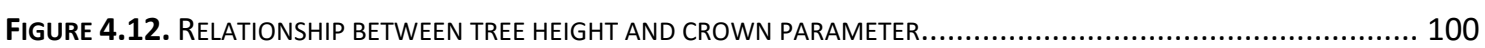

FIGURE 4.13. HEIGHT-DIAMETER RELATIONSHIP AND VERTICAL LEAF AREA DENSITY DISTRIBUTION........................... 101

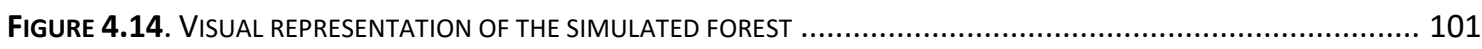

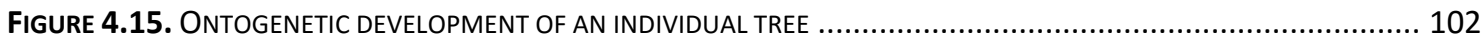

Figure 4.16. TREE DYNAMICS IN DEPENDENCE OF THEIR TRAITS AND THE ENVIRONMENTAL CONDITIONS ..................... 103

Figure 4.17. MAXIMUM TREE heIGHT AS A FUnCTION OF TREE TRAITS AND ENVIRONMENTAL CONDITIONS ................... 104

FIGURE 4.18. LIGHT COMPENSATION POINT OF A LEAF COMPARTMENT IN DEPENDENCE ON SLA AND THE PIPE LENGTH ..... 105

FiguRE 5.1. GENERATION OF MICROHABITAT MATRICES BASED ON THE RESULT OF THE FOREST MODEL ........................ 121

FIGURE 5.2. FLOWCHART OF THE COUPLED FOREST-EPIPHYTE MODEL. ..................................................... 123

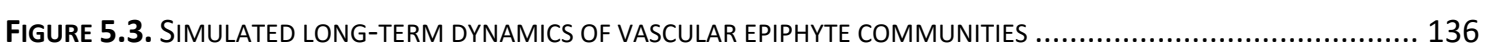


FIGURE 5.4. RANK-ABUNDANCE CURVES AND VERTICAL DISTRIBUTIONS OF EPIPHYTES.

FIGURE 5.5. VERTICAL STRATIFICATION OF SPECIES IN OUR MODEL IN COMPARISON TO DATA FROM PANAMA AND ECUADOR 138

Figure 5.6. SizE-DISTRIBUTIONS IN EPIPHYTE COMMUNITIES: (A) MODEL, (B) PANAMA, (C) ECUADOR. 138

FIGURE 5.7. EFFECTS OF DIFFERENCES IN FOREST DYNAMICS, LOGGING REGIMES AND FRAGMENT SIZES ON ABUNDANCES, SPECIES NUMBERS AND OCCUPIED SUBSTRATE AREAS OF EPIPHYTE ASSEMBLAGES. 140

FIGURE A.1. PHOTOGRAPH OF THE STUDY SITE AND VERTICAL LIGHT INTENSITY GRADIENT ..................................... 181

Figure A.2. RELATIONSHIP BETWEEN hEIGHT ABOVE GROUND AND SIX LEAF TRAIT 182

FigURE A.3. TRAIT-HEIGHT RELATIONSHIPS FOR FOUR TAXONOMIC GROUPS OF VASCULAR EPIPHYTES (AROIDS, BROMELIADS, ORCHIDS AND FERNS) 183

FigURE A.4. INTRASPECIFIC TRAIT-HEIGHT RELATIONSHIP BETWEEN SPECIFIC LEAF AREA AND HEIGHT ABOVE GROUND ...... 184

FigURE A.5. INTRASPECIFIC TRAIT-HEIGHT RELATIONSHIP BETWEEN LDMC AND HEIGHT ABOVE GROUND.......................185

FIGURE A.6. INTRASPECIFIC TRAIT-HEIGHT RELATIONSHIP BETWEEN LEAF THICKNESS AND HEIGHT ABOVE GROUND............ 186

FigURE A.7. INTRASPECIFIC TRAIT-HEIGHT RELATIONSHIP BETWEEN LEAF WATER CONTENT AND HEIGHT ABOVE GROUND .. 187

FIGURE A.8. INTRASPECIFIC TRAIT-HEIGHT RELATIONSHIP BETWEEN LEAF CHLOROPHYLL CONTENT PER LEAF DRY MASS AND HEIGHT ABOVE GROUND 188

FigURE A.9. INTRASPECIFIC TRAIT-HEIGHT RELATIONSHIP BETWEEN LEAF CHLOROPHYLL CONTENT PER LEAF AREA AND HEIGHT ABOVE GROUND 189

Figure A.10. PARTITIONING OF TOTAL TRAIT VARIANCE INTO BETWEEN-SPECIES (INTERSPECIFIC) VARIANCE AND WITHINSPECIES (INTRASPECIFIC) VARIANCE FOR SIX LEAF TRAITS 190

FIGURE A.11. RELATIONSHIP BETWEEN INTRASPECIFIC TRAIT VARIABILITY AND VERTICAL RANGE OF EPIPHYTE SPECIES IN A PANAMANIAN LOWLAND FOREST. 190

Figure C.1. EFFECTS OF COMPETITION ON TREE GROWTH 202

FIGURE D.1. LONG-TERM DYNAMICS OF THE SIMULATED FOREST STANDS DIFFERING IN NATURAL FOREST DYNAMICS USED AS MODEL SCENARIOS IN THIS STUDY 216

FIGURE D.2. LONG-TERM DYNAMICS OF SIMULATED FOREST STANDS DIFFERING IN LOGGING INTENSITIES 217

FIGURE D.3. LONG-TERM DYNAMICS OF SIMULATED FOREST STANDS DIFFERING IN FRAGMENT SIZE 218

FIGURE D.4. SIMULATED LONG-TERM DYNAMICS OF VASCULAR EPIPHYTES COMMUNITIES 219

FIGURE D.5. COMMUNITY GROWTH RATES AND PARTITIONING OF MORTALITY RATES. 219

FigURE D.6. VERTICAL DISTRIBUTION OF EPIPHYTES 220

FIGURE D.7. 3D STRUCTURE OF EPIPHYTE ASSEMBLAGES 


\section{Author contributions}

Chapter 2 - Functional leaf traits of vascular epiphytes: vertical trends within the forest, intra- and interspecific trait variability, and taxonomic signals

Gunnar Petter, Katrin Wagner, Wolfgang Wanek, Eduardo Javier Sánchez Delgado, Gerhard Zotz, Juliano Sarmento Cabral and Holger Kreft

GZ conceived the study; ES, KW and GZ designed the methodology for data collection; ES collected the data with contributions by GP and JS; WW analyzed leaf samples for nitrogen content and isotope ratios; GP and KW decided upon the exact research questions and methods for data analysis and analyzed the data; GP led the writing with substantial contributions from KW, JSC, HZ and HK.

published in Functional Ecology, 2016, 30, 188-198, DOI: 10.1111/1365-2435.12490

Chapter 3 - Branchfall as a demographic filter for epiphyte communities: Lessons from forest floor-based sampling

Juliano Sarmento Cabral, Gunnar Petter, Glenda Mendieta-Leiva, Katrin Wagner, Gerhard Zotz and Holger Kreft

JSC, GP, GZ and HK conceived and designed the experiments; JSC and GP performed the experiments and analyzed the data; GZ, GML and KW contributed additional data and analysis tools; JSC led the writing with substantial contributions from GP, GZ and HK.

published in PLoS One, 2015, 10, e0128019, DOI:10.1371/journal. pone.0128019

Chapter 4 - Modelling the long-term dynamics of tropical forests using functional-structural tree models: Leaf trait trade-offs as predictor of whole-tree life history variations

Gunnar Petter, Yongzhi Ong, Gerhard Zotz, Holger Kreft, Juliano Sarmento Cabral

GP, JSC and HK conceived the idea; GP conceptualized and programmed the model, with contributions from YO; GP validated and analyzed the model; GP lead the writing with assistance from JSC, HK and GZ. 
Chapter 5 - Simulating the influence of forest dynamics on structure and dynamics of epiphyte assemblages based on a coupled forest-epiphyte model

Gunnar Petter, Gerhard Zotz, Holger Kreft, Juliano Sarmento Cabral

GP, JSC, GZ and HK conceived the idea; GP conceptualized and programmed the model; GP validated and analyzed the model; GP lead the writing with assistance from JSC, HK and GZ. 


\section{Summary}

Vascular epiphytes are non-parasitic plants that germinate and grow on trees without contact to the soil. Their arboreal life style implies a strong dependence on forest structure and dynamics. Tree architectures change constantly during ontogeny, and large and old branches of the inner crowns are generally more suitable for epiphyte colonization and survival than small branches of the outer crowns. In addition, microclimatic conditions within canopies, such as light, temperature or humidity, are directly influenced by forest structure. While the influence of such gradients within trees and forests on the vertical distribution of epiphyte species is undisputed, our understanding of the relationship between epiphyte distribution and functional traits is limited. Moreover, a causal relationship between the dynamics of trees and forests and the dynamics of epiphyte assemblages is obvious, but our quantitative knowledge on this topic is strikingly scarce.

In this thesis, I provide a detailed analysis of how forest structure and dynamics influence the structure and dynamics of epiphyte assemblages and their functional traits via both field studies (chapters 2 and 3) and modelling studies (chapters 4 and 5).

In chapter 2, I analyzed vertical gradients of ten leaf traits based on leaf samples of $>1100$ individuals belonging to 83 epiphyte species in a Panamanian lowland forest. I found that community mean trait values of many leaf traits were significantly correlated with height above ground. Trait-height correlations were particularly strong for specific leaf area (SLA), leaf thickness, leaf chlorophyll concentration and carbon isotope ratio. Both linear and non-linear trends were observed, and while the leaf thickness, for instance, increased linearly, the SLA decreased non-linearly with height. Furthermore, intraspecific trait variability was pronounced and accounted for one-third of total observed trait variance. Intraspecific trait adjustments along the vertical gradient were common and seventy per cent of all species showed significant traitheight relationships. In addition, intraspecific trait variability was positively correlated with the vertical range occupied by species; however, this correlation was rather weak. I also observed significant trait differences between major taxonomic groups (orchids, ferns, aroids, bromeliads) that were linked to their vertical distributions. Orchids, for instance, had on average the thickest leaves and lowest SLA values, while in ferns the leaf dry matter content was almost twofold higher than in the other taxonomic groups. My study represents the most comprehensive study on vertical trait gradients of vascular epiphytes to date and demonstrates that leaf trait syndromes and intraspecific trait variability play important roles in explaining the vertical zonation of vascular epiphyte species and taxonomic groups. 
In chapter 3, I addressed the role of forest dynamics on community structure and mortality patterns of epiphyte assemblages by exploring the forest floor as source of information. To this end, I surveyed fallen branches and epiphytes in 96 transects in rainforests in Brazil and Panama. I found that trends in epiphyte abundance, richness and composition over branch diameter on the forest floor reflected trends in the forest canopy. This finding suggests that forest floor surveys provide useful demographic information, particularly on epiphytes occurring on the thinnest branches which are least accessible with the most common techniques to access the forest canopy. Furthermore, the density of epiphytes on the forest floor was high, and I estimated mortality rates of at least $4 \%$ per year at the community level, and of $\sim 13 \%$ per year when considering epiphytes on branches $<10 \mathrm{~cm}$ in diameter. The results of this study highlight the importance of tree and forest dynamics for the demography of vascular epiphytes.

In chapter 4, I developed a dynamic forest stand model in which trees are represented by their three-dimensional (3D) structure. In this model, tree species were characterized by a set of leaf traits under consideration of trade-offs and correlations among traits. Applying the principles of the pipe model theory, these leaf trait trade-offs were scaled to whole-tree growth. This approach reproduced fundamental life history variation between different functional tree groups with regard to their growth, survival, and light demand. For instance, species with high SLA values had high initial growth rates, but lower maximum heights and shorter life spans, i.e. characteristics associated with pioneer species. Tree growth patterns in my model were largely consistent with observations and support the notion that the growth-survival trade-off across tropical tree species is, at least partly, determined by leaf traits. Furthermore, I coupled the trait-based tree model with a forest stand model which simulates key demographic processes and integrates between-tree competition. This stand model successfully reproduced a number of important ecological patterns. A dynamic equilibrium state was reached after $\sim 100$ years, and in this equilibrium twelve important forest attributes (e.g. above-ground biomass, basal area, stem number, net-primary production or leaf area index) were within typical ranges of Neotropical lowland forests. Moreover, complex patterns like the vertical leaf area density or the diameter-height relationship closely matched observations, indicating that a structurally realistic forest can be simulated with my model. To my knowledge, the presented modelling approach allowing detailed 3D long-term simulations of forest dynamics is unique and paves the way for further model-based analyses of forest dynamics, or model-based studies of canopy-dwelling organisms requiring a detailed representation of forest structures and their dynamics.

In chapter 5, I developed the first mechanistic model for epiphytes which explicitly simulates population dynamics while being coupled with a structurally-realistic forest model. This epiphyte model is three-dimensional, spatially-explicit, and trait- and individual-based. After the model was validated by comparing model results with field data, I used simulation experiments to assess 
how differences in natural forest dynamics, logging strategies, and the size of forest patches influenced the long-term dynamics of epiphyte assemblages. Tree turnover rates in natural tropical rainforest typically vary between $1 \%$ and $3 \%$ per year, and such variations had a marked impact on epiphyte assemblages, i.e. forests with low tree turnover rates had considerably lower extinction rates and higher epiphyte abundances. It has been observed that even in mature forests with low tree turnover rates, epiphyte assemblages show no sign of saturation, and my simulations demonstrated that the saturation level was clearly influenced by forest dynamics. Furthermore, logging had the hypothesized negative effect on epiphyte diversity and abundance. Strikingly, a slight reduction in size of logged trees from 45 to $40 \mathrm{~cm}$ in diameter at breast height had a catastrophic effect on epiphyte assemblages and resulted in nearly complete extinction. In contrast, epiphyte extinction rates decreased with increasing forest patch sizes. The coupled epiphyte-forest model presented in this study provided valuable insights on how forests stand parameters influence epiphyte assemblages and has the potential to address pending question in the field of epiphyte ecology and conservation in future studies.

In summary, the findings of my thesis represent a major advance towards a better understanding of the relationship between forest structure and dynamics and (trait) structure and dynamics of epiphyte communities. My thesis constitutes the most comprehensive study on the community trait structure of vascular epiphytes to date and introduced complex mechanistic models to the field of epiphyte ecology. The modelling approaches open new avenues for future studies of spatial and temporal dynamics of vascular epiphyte assemblages while integrating epiphyte research in a more theoretical context. 



\section{Introduction}

\section{The epiphytic life style}

Vascular epiphytes are non-parasitic plants that germinate and grow on other plants without contact to the soil (Zotz 2013). Their arboreal life allows them to reach the well-illuminated upper strata of forests without substantial investments in plant structure (Benzing 1990). However, being isolated from terrestrial soils, epiphytes have to cope with a low and irregular supply of water and nutrients from atmospheric inputs, litter or canopy soils (Benzing 1990; Wania, Hietz \& Wanek 2002). Particularly water availability is a key factor in epiphytic habitats, and diversity and abundance generally increases strongly with precipitation and humidity (Gentry \& Dodson 1987; Kreft et al. 2004; Ding et al. 2016).

Epiphytes are a conspicuous and important component of rain forests in the tropics and subtropics (Schimper 1888; Gentry \& Dodson 1987; Benzing 1990). In fact, in wet montane forests their species number can even exceed that of non-epiphytic species (Foster 2001; Kelly et al. 2004). In a Peruvian cloud forest, a single tree hosted the remarkable number of 190 epiphyte species (Catchpole \& Kirkpatrick 2010). However, their distribution is not restricted to low-latitude regions, and some temperate forests in both the northern and southern hemisphere also harbor rich epiphyte floras (Zotz 2005; Burns \& Dawson 2005).

With more than 27,000 described species, vascular epiphytes represent $\sim 9 \%$ of the world's vascular plant diversity (Zotz 2013). This plant group is taxonomically diverse and 913 genera in 73 families have epiphytic members (Zotz 2013). However, epiphyte species are not evenly distributed among taxa. Orchids are by far the largest group accounting for $\sim 68 \%$ of all epiphytes, but ferns and fern allies $(\sim 10 \%)$ as well as bromeliads $(\sim 6 \%)$ are also important. These structurally dependent plants do not only contribute to local diversity, they can also have a positive effect on forest ecosystem processes by modifying micro-environmental conditions (Stanton et al. 2014). In addition, epiphyte associations with fauna are not uncommon. For example, the tanks of bromeliads can provide microhabitats for a number of invertebrates and amphibians (Stuntz et al. 2002; Yanoviak, Nadkarni \& Solano J. 2007). In spite of their importance, many aspects of epiphyte ecology are still not well studied, particularly when compared to other plant groups (Kitching 2006; Mendieta-Leiva \& Zotz 2015). While there are numerous works on distribution and floristic composition of epiphytes, studies in the field of trait-based ecology are rare. Our knowledge on structure and dynamics of epiphyte populations and assemblages is similarly limited (Mendieta-Leiva \& Zotz 2015). 


\section{Introduction}

\section{Spatial structure of epiphyte assemblages}

The non-random spatial distribution of epiphyte species within forest stands and on individual trees is a striking characteristic of epiphyte assemblages, and usually a pronounced vertical stratification is observed (Schimper 1888; Zotz 2007; Zotz \& Schultz 2008). Some filmy fern species are almost exclusively found at the lower trunk bases (Krömer \& Kessler 2006), others such as tiny twig epiphytes predominantly occupy the outer crowns (Chase 1987), but the majority of species colonizes vertical ranges of different extension between these extremes (Krömer, Kessler \& Gradstein 2007). Within-tree distribution of substrate and microclimatic conditions are considered as main reason for the spatial structure of epiphyte assemblages (Benzing 1990; Zotz 2007). In fact, conditions vary widely from the humid and shady lower trunks, which are available for colonization over longer time periods, to the well-illuminated, drier and highly dynamic outer crowns. Such heterogeneity in conditions is reflected in the epiphyte assemblage by the presence of a large number of ecologically and functionally diverse species.

Functional diversity can be assessed by investigating functional traits, which are characteristics of plants that affect their growth, reproduction and survival (Violle et al. 2007). Therefore, a correlation between functional traits and the vertical position of epiphytes can be assumed. Few studies addressed this topic and compared epiphytes from distinct pre-defined zones within trees (Andrade \& Nobel 1997; Hietz \& Briones 1998) or within the forest (Mantovani 1999; Stuntz \& Zotz 2001). Stuntz \& Zotz (2001), for instance, observed that epiphytes in the forest canopy had lower specific leaf areas (SLA) but higher photosynthetic capacities compared to understory epiphytes. Likewise, Hietz \& Briones (2001) observed a correlation between the leaf nitrogen content and the position in the tree crown. These studies indicate that some traits are indeed related to their vertical position, however, Zotz (2007) pointed out that height above ground might be more suitable to approximate the environmental gradients within forests than pre-defined zones. Accordingly, not only trait differences between different zones but vertical trait gradients can be expected. To our knowledge, only a single study has analyzed the trait-height relationships for epiphytes so far, finding a significant linear decrease in SLA with height (Cavaleri et al. 2010). However, this correlation was weaker than that in other plant groups included in the same study. As this study focused on a single trait and did not include epiphytes from important taxonomic groups such as orchids or bromeliads, many aspects of the vertical trait distribution of epiphytes are thus still largely unexplored and unknown. Studies on soil-rooted plants, for instance, also highlighted the importance of intraspecific trait variability (Albert et al. 2010b, 2012; Bolnick et al. 2011). High intraspecific trait variability might be associated with a large ecological breadth or ecological generalism in plants (van Valen 1965; Sides et al. 2014). For epiphytes, high intraspecific trait variability may thus enable a high vertical range within forests, but this remains 
to be adequately addressed. Hence, there are a number of open questions in the field of trait-based ecology of vascular epiphytes which warrant more attention.

\section{Influence of forest dynamics on epiphyte dynamics}

Forest structure and dynamics not only influence the spatial structure and trait distribution of epiphyte assemblages, but also their dynamics (Zotz, Bermejo \& Dietz 1999). Each tree is a living organism whose architecture experiences substantial modification during ontogeny, as new structural biomass is continuously generated by growth processes, but also lost via branch shedding (Hallé, Oldeman \& Tomlinson 1978; Barthélémy \& Caraglio 2007). Small trees in the understory of tropical forests are usually characterized by slender trunks and few horizontal branches which are shed frequently (Millington \& Chaney 1973; Addicott 1991; Alves \& Santos 2002). Such trees are poor phorophytes (i.e. host trees) and thus often free of epiphytes (Taylor \& Burns 2015). Large trees, in contrast, provide greater substrate areas that are available for colonization for a longer period, and epiphyte richness and abundance thus generally increases with tree size (Laube \& Zotz 2006; Taylor \& Burns 2015). However, each branch and tree eventually falls (Meer et al. 1996), carrying their epiphytes with them to the forest floor. The survival of epiphytes on the forest floor is limited (Matelson, Nadkarni \& Longino 1993) and successful reproduction is virtually impossible. Hence, these individual are lost from the community. In the studies by Hietz (1997) and by Zotz, Laube \& Schmidt (2005), substrate failure was the single most common cause of epiphyte mortality, which emphasizes the importance of tree and forest dynamics for epiphyte dynamics.

Quantitative studies on the dynamics of epiphyte assemblages are, however, rare. Apart from the study by Hietz (1997) who used repeated photographs to monitor epiphytes on branch sections, there are two studies that assessed temporal changes on certain host tree species (Socratea exorrhiza: Laube \& Zotz 2006; Annona glabra: Zotz, Bermejo \& Dietz, 1999), and two studies with repeated censuses at the plot scale (1 ha plot in Venezuela: Schmit-Neuerburg 2002; 0.4 ha plot in Panama: first census by Zotz \& Schultz 2008; second census by G. Mendieta-Leiva, K. Wagner \& G. Zotz, unpublished data). Interestingly, all these studies found increasing abundances, which suggests that epiphyte communities are unsaturated. However, Zotz \& Schultz (2008) also reported that a single large tree hosted almost $15 \%$ of all epiphytes in their 0.4 ha plot; such trees disproportionately abundant with epiphytes will ultimately die and this leads to pronounced local losses of epiphytes. This suggests that tree turnover rates, which commonly vary between $1 \%$ and $3 \%$ per year in tropical rainforests when considering trees $>10 \mathrm{~cm}$ in DBH (Phillips 1996; Phillips et al. 2004b; Lewis et al. 2004b), should be important determinants of local epiphyte abundance and diversity in addition to water-related climatic variables (e.g. Kreft 


\section{Introduction}

et al. 2004; Ding et al. 2016). Moreover, an alarming increase in tree turnover rates in tropical forests over the last decades has been observed (Phillips 1996; Lewis et al. 2004b). Thus, there is an urgent need to improve our understanding of the influence of forest dynamics on epiphyte dynamics. Appropriate field studies, however, are tedious; forest canopy research requires laborintensive or costly techniques (e.g. rope-climbing, canopy cranes), which makes it difficult to gather information on community structure and dynamics. Therefore, complementary methods are urgently needed if we are to understand the dynamics of vascular epiphytes.

\section{Mechanistic models of epiphyte dynamics}

Mechanistic model approaches provide an opportunity to improve our knowledge on ecological systems in addition to field studies. They can help to disentangle complex interactions and to predict future changes (Wiegand et al. 2003; Purves \& Pacala 2008). However, mechanistic models for epiphytes have not been developed so far, precluding any ecological modeling assessment for these important components of the world's flora. Nevertheless, virtual forests, in which simulation experiments on epiphyte demography can be conducted, provide a promising starting point to include vascular epiphyte in the ecological modeling agenda.

Among the available methods to generate virtual forests, functional-structural tree models (FSTMs) simulate the three-dimensional dynamics of plant structures and have attracted increasing attention in recent times (Barczi et al. 2008; DeJong et al. 2011). In these models, trees can be represented by a large number of individual branch segments, leaves or reproductive organs (Sterck et al. 2005; Barczi et al. 2008). By combining function and structure, FSTMs allow an integration of the main processes affecting tree growth, i.e. resource capture and within-tree allocation, at a high level of detail, for instance by simulating the interactions between leaf distribution and light conditions within individual trees (Sterck et al. 2005; Fourcaud et al. 2008). FSTMs have mainly been applied to simulate and analyze growth of individual trees (Perttunen, Sieva \& Nikinmaa 1998; Sterck et al. 2005; Sterck \& Schieving 2007), and only few attempts have been made to couple FSTMs with forest stand models (Feng et al. 2011; Guillemot et al. 2014). In the latter cases, the forest models focused on even age-stands in single species systems over a limited time frame, but to our knowledge there is no long-term dynamic stand model based on FSTMs including all demographic processes.

Three-dimensional dynamic stand models, which are able to reproduce detailed processes such as branch turnover or gap dynamics, could function as virtual laboratories for epiphytes communities. Simulation experiments going beyond the scope of field studies could be conducted, for instance by modelling epiphyte dynamics over several hundred years. Moreover, Mendieta- 
Leiva \& Zotz (2015) mentioned that the study of the structure and dynamics of epiphyte assemblages lacks theory, and modelling studies have a great potential to advance this field of research.

\section{Study outline}

This thesis aims at improving our understanding of the trait-based ecology of vascular epiphytes and of how forest structure and dynamics influence the structure and dynamics of epiphyte assemblages. The specific objectives are to (1) analyze vertical trends of functional leaf traits of vascular epiphytes within forests and to assess the importance of intraspecific trait variability in epiphyte systems, (2) document epiphyte mortality as a function of branch or tree fall, (3) develop a detail three-dimensional forest model to simulate long-term forest dynamics, which can be coupled with a demographic epiphyte model to (4) assess how natural or human-induced differences in long-term forest dynamics affect the structure and dynamics of epiphyte assemblages. This thesis comprises field studies (chapters 2 and 3) and modelling studies (chapters 4 and 5).

In chapter 2, I analyze trait-height relationships at the community and the species level for a number of leaf traits of vascular epiphytes. To this end, samples are collected along the vertical gradient in a Panamanian lowland forest using a canopy crane. I quantify the importance of intraspecific trait variability and investigate whether vertical ranges of species correlate with their trait variability. Moreover, I test if traits and trait syndromes differ among important taxonomic groups of epiphytes (bromeliads, orchids, aroids, ferns).

In chapter 3, I address the role of the forest floor as source of information on epiphyte mortality and community structure. I compare patterns of fallen branches and epiphyte between two study sites and assess the impact of branch turnover on epiphyte mortality.

In chapter 4, I develop a dynamic forest stand model in which each tree is represented by its threedimensional structure. This model is used to simulate the long-term forest dynamics (500-1000 years) at the plot scale ( $\sim 1$ ha) at a high degree of detail. I characterize each tree by a set of leaf traits under consideration of between-trait trade-offs and correlations and hypothesize that this trait-based approach will reproduce life history variation between different functional groups with regard to their growth, survival, and light demand. I use multiple observed patterns at the tree and forest level to parameterize and validate the model. 


\section{Introduction}

In chapter 5, I couple the forest stand model with an individual-based model of vascular epiphytes and assess how (1) differences in natural forest dynamics, (2) selective logging and (3) the size of forest patches influence the long-term dynamics of epiphyte assemblages. 
2 Functional leaf traits of vascular epiphytes: vertical trends within the forest, intra- and interspecific trait variability, and taxonomic signals

Gunnar Petter, Katrin Wagner, Wolfgang Wanek, Eduardo Javier Sánchez Delgado, Gerhard Zotz, Juliano Sarmento Cabral and Holger Kreft

published in Functional Ecology, 2016, 30, 188-198, DOI: 10.1111/1365-2435.12490 


\subsection{Summary}

1. Analyzing functional traits along environmental gradients can improve our understanding of the mechanisms structuring plant communities. Within forests, vertical gradients in light intensity, temperature and humidity are often pronounced. Vascular epiphytes are particularly suitable for studying the influence of these vertical gradients on functional traits because they lack contact with the soil and thus individual plants are entirely exposed to different environmental conditions, from the dark and humid understory to the sunny and dry outer canopy.

2. In this study, we analyzed multiple aspects of the trait-based ecology of vascular epiphytes: shifts in trait values with height above ground (as a proxy for vertical environmental gradients) at community and species level, the importance of intra- vs. interspecific trait variability, and trait differences among taxonomic groups. We assessed ten leaf traits for 1,151 individuals belonging to 83 epiphyte species of all major taxonomic groups co-occurring in a Panamanian lowland forest.

3. Community mean trait values of many leaf traits were strongly correlated with height and particularly specific leaf area and chlorophyll concentration showed non-linear, negative trends.

4. Intraspecific trait variability was pronounced and accounted for one third of total observed trait variance. Intraspecific trait adjustments along the vertical gradient were common and seventy percent of all species showed significant trait-height relationships. In addition, intraspecific trait variability was positively correlated with the vertical range occupied by species.

5. We observed significant trait differences between major taxonomic groups (orchids, ferns, aroids, bromeliads). In ferns, for instance, leaf dry matter content was almost twofold higher than in the other taxonomic groups. This indicates that some leaf traits are taxonomically conserved.

6. Our study demonstrates that vertical environmental gradients strongly influence functional traits of vascular epiphytes. In order to understand community composition along such gradients, it is central to study several aspects of trait-based ecology, including both community and intraspecific trends of multiple traits. 


\section{Functional leaf traits of vascular epiphytes}

\subsection{Introduction}

Functional traits are measurable characteristics of individual plants impacting their growth, reproduction and survival (Violle et al. 2007). The analysis of functional traits along environmental gradients can help to unravel the mechanisms structuring plant communities (Wright et al. 2005b; Ackerly \& Cornwell 2007). Significant shifts in community mean trait values, for instance, indicate trait-based environmental filtering (Díaz, Cabido \& Casanoves 1998; Cornwell \& Ackerly 2009). Interestingly, studies based on global trait datasets show that largescale changes in climatic conditions only explained a small proportion of observed variation in leaf traits, while trait variation among co-existing species within study plots was relatively high (Wright et al. 2004, 2005b). In fact, the environment at small scales can be very heterogeneous, promoting the occurrence of species with different traits and ecological strategies. Moreover, particularly in forests, environmental factors such as light intensity, temperature and humidity normally show marked vertical gradients. Such vertical gradients, in turn, have the potential to explain a substantial part of trait variations at plot scale, and it has been demonstrated that several leaf traits of trees change significantly along vertical light gradients (Rozendaal, Hurtado \& Poorter 2006; Markesteijn, Poorter \& Bongers 2007).

Vascular holoepiphytes, plants growing non-parasitically on other plants without contact to the soil (Zotz 2013), are particularly suitable for studying the influence of vertical environmental gradients on functional traits, because individuals are entirely exposed to different environmental conditions from the dark and humid understory to the sunny and dry outer canopy. As the leaf weight ratio (leaf mass/total plant mass) is generally high in epiphytes (Zotz \& Asshoff 2010), leaf traits should be pivotal to their performance. The frequently pronounced vertical stratification of epiphyte species has long been recognized (Schimper 1888; Krömer et al. 2007), but few studies have attempted to relate their vertical distribution to functional leaf traits. Most of these studies assessed differences between sun and shade plants (e.g. Mantovani 1999) or used predefined zones within forests or trees (e.g. Johansson zones; Johansson 1974) as surrogates for different environmental conditions (Andrade \& Nobel 1997; Hietz \& Briones 1998; Stuntz \& Zotz 2001). Zotz (2007) pointed out that height above ground might be more suitable to approximate the environmental gradients within forests than predefined zones. To our knowledge, only a single study related height above ground to leaf traits of vascular epiphytes (Cavaleri et al. 2010). However, as this study focused on leaf mass per area (LMA) and did not include epiphytes from important taxonomic groups like orchids or bromeliads, many aspects of the vertical leaf trait distribution of epiphytes are still largely unexplored.

Along vertical environmental gradients, shifts in community mean trait values of functionally important leaf traits can be expected. An increase in specific leaf area $\left(\mathrm{SLA}=\mathrm{LMA}^{-1}\right)$, for instance, increases the light-capture efficiency, which is advantageous under low-light conditions in the 
understory (Wright et al. 2004). In contrast, an increase in leaf thickness can prevent overheating and minimize transpiration losses, which is favorable under drier und sunnier conditions in the canopy (Cornelissen et al. 2003; Rozendaal et al. 2006). Such shifts in community trait means might be caused by replacement of species with unsuitable traits. However, recent studies have also highlighted that intraspecific trait variability can be quite substantial and that individuals within species can adjust their traits in response to the environment (Bolnick et al. 2011; de Bello et al. 2011; Kichenin et al. 2013). Additionally, high intraspecific trait variability might be associated with a large ecological breadth or ecological generalism, possibly increasing the vertical range of epiphytes within forests (van Valen 1965; Sides et al. 2014).

Vascular epiphytes are a taxonomically diverse group. Orchids account for $68 \%$ of all epiphyte species, but ferns and lycophytes, bromeliads and aroids are also prominent taxa (Zotz 2013). It is generally assumed that traits are taxonomically conserved and, consequently, trait differences between taxonomic groups can be expected. Moreover, epiphyte taxa independently evolved a variety of different morphological and physiological characteristics (e.g. velamen radicum, phytotelmata, specialized trichomes) to cope with nutrient and water limitation (Benzing 1990). Such between-taxon differences might affect the response of leaf traits to environmental conditions.

To analyze the multiple aspects of trait-based ecology along vertical environmental gradients, we studied ten leaf traits for 1,151 individuals of 83 epiphyte species of all major taxonomic groups co-occurring in a Panamanian lowland forest. We tested the following hypotheses: (H1) trait means and trait syndromes change with height at the community level; $(\mathrm{H} 2)$ variations in traitheight relationships among species influence community trait structure; $(\mathrm{H} 3)$ vertical ranges of epiphyte species correlate with their intraspecific trait variability; (H4) trait means, trait syndromes and trait-height relationships differ among taxonomic groups.

\subsection{Materials and methods}

\section{Study site}

This study was conducted at the San Lorenzo Canopy Crane Site at the Atlantic coast of Panama $\left(9^{\circ} 17^{\prime} \mathrm{N}, 79^{\circ} 58^{\prime} \mathrm{W}, 130 \mathrm{~m}\right.$ a.s.l.; Wright et al. 2003). Mean annual precipitation in this old-growth lowland tropical rainforest is around 3,100 $\mathrm{mm}$, with a pronounced dry season from January to March. Canopy height is variable and emergent trees reach maximum heights of ca. $45 \mathrm{~m}$. The use of a gondola attached to a construction crane allowed access to all strata of the forest within an area of ca. 0.9 ha. A comprehensive census of the vascular epiphyte flora at the study site was conducted in 2010 - 2012 and yielded > 22,000 individuals of $>100$ species (Glenda MendietaLeiva \& Gerhard Zotz, unpublished data; see Zotz \& Schultz 2008 for methodology). 


\section{Functional leaf traits of vascular epiphytes}

Among vertical environmental gradients, the light gradient is considered as most influential on leaf traits (e.g. Poorter 1999; Markesteijn, Poorter \& Bongers 2007). Changes in light intensity with height above ground were measured in situ with light intensity loggers (HOBO UA-002-64; Onset Computer Corporation, Cape Cod, USA; for more details see Fig. A.1 in Appendix).

\section{Leaf traits}

As we focused on multiple aspects of trait-based ecology (e.g. community and intraspecific trends), we applied a two-tiered sampling strategy. First, we randomly sampled epiphytes along the vertical gradient within the entire area accessible by crane to represent the epiphyte community. Second, for species which were quite frequent in the study area (based on the census), but underrepresented in our sample, we additionally increased the sample size to $n=10$, which we regarded as minimum to analyze intraspecific trends. However, this applied to only few species and thus should not bias community trends.

We collected one leaf per epiphyte for 1,151 individuals belonging to 83 species ( 51 species $\geq 10$ samples) in 15 plant families (Table A.1). For each sampled individual, height above ground was recorded. We sampled adults and juveniles, but not seedlings. The juvenile phase can last several years in epiphytes, and by sampling these individuals, we were able to include more species in our analysis. However, we note that including juveniles can lead to increased intraspecific trait variability ( $\sim 25 \%$ of all individuals were juveniles; $\sim 60 \%$ of all species included juveniles). We aimed at sampling the youngest, fully expanded leaves, without signs of herbivory or infections. The taxonomic nomenclature used in the present paper follows The Plant List (2014; http://www.theplantlist.org/).

For each sample, we determined specific leaf area (SLA = leaf area / dry weight; $\mathrm{mm}^{2} \mathrm{mg}^{-1}$ ), leaf dry matter content $\left(\mathrm{LDMC}=\right.$ dry weight $/$ fresh weight; $\left.\mathrm{g} \mathrm{g}^{-1}\right)$, leaf lamina thickness (Thickness; $\mathrm{mm})$, leaf water content on an area basis $\left(\mathrm{LWC}_{\text {area }}=(\right.$ fresh weight - dry weight $) /$ leaf area; $g$ $\left.\mathrm{H}_{2} \mathrm{O} \mathrm{m}^{-2}\right)$, as well as leaf chlorophyll concentration on an area basis $\left(\mathrm{Chl}_{\text {area }} ; \mu \mathrm{g} \mathrm{cm}^{-2}\right)$ and on a mass basis $\left(\mathrm{Chl}_{\text {mass }} ; \mathrm{mg} \mathrm{g}^{-1}\right)$. Chlorophyll concentration was estimated by measuring red/infrared absorbance in the field with a SPAD-502 chlorophyll meter (Spectrum Technologies, Plainfield, USA). SPAD measurements were converted into chlorophyll concentrations using the general relationship from Coste et al. (2010). Collected leaves were re-watered with deionized water for $>6 \mathrm{~h}$ before taking additional measurements. Leaf thickness was measured with a caliper (precision: $0.05 \mathrm{~mm}$ ). Each leaf was photographed and leaf area was determined in Adobe Photoshop 6.0 (Adobe Systems, San Jose, USA). Leaves were weighed to obtain fresh weight (balance: A\&D GR-202; A\&D Company, Tokyo, Japan; precision: $0.1 \mathrm{mg}$ ), oven dried at $70{ }^{\circ} \mathrm{C}$ for $48 \mathrm{~h}$, and re-weighed to obtain dry weight. 
Additionally, for a sub-sample (224 individuals of 61 species), leaf nitrogen concentration on both mass $\left(\mathrm{N}_{\text {mass }} ; \mathrm{mg} \mathrm{g}^{-1}\right)$ and area basis $\left(\mathrm{N}_{\text {area }} ; \mathrm{g} \mathrm{m}^{-2}\right)$, as well as nitrogen isotope $\left(\delta^{15} \mathrm{~N} ; \%\right)$ and carbon isotope ratios $\left(\delta^{13} \mathrm{C} ; \%\right.$ ) were determined after homogenization of the dried samples in a ball mill by elemental analyzer-isotope ratio mass spectrometry (Delta PLUS; Thermo Electron, Bremen, Germany). As universal standards, atmospheric air was used for ${ }^{15} \mathrm{~N}$ and the Vienna Pee Dee Belemnite for ${ }^{13} \mathrm{C}$. In the following, we will refer to the traits of this sub-sample as nitrogencarbon (NC) traits.

\section{Data analyses}

Analyses were done in R 3.0.1 (R Development Core Team 2013). Analyses for each hypothesis are described separately in the following.

\section{H1 - Trait means and trait syndromes change with height at the community level}

We used simple linear models (LMs) to analyze the relationship between leaf traits and height. To test for non-linearity, simple LMs (trait $\sim$ height) and LMs including a quadratic term (trait height + height ${ }^{2}$ ) were fitted and compared using the Akaike Information Criterion (AIC). Choosing a conservative approach, we selected the non-linear model as minimal adequate model (MAM) if it received higher model support by $\triangle \mathrm{AIC}>10$ (Burnham \& Anderson 2004). For each trait, LMs were applied to the entire dataset consisting of all sampled individuals, as well as to community trait means calculated for all $1-\mathrm{m}$ height intervals. CAM species, defined by $\delta^{13} \mathrm{C}$ values $>-20 \%$ (compare Zotz 2004), were excluded from analysis of vertical trends in $\delta^{13} \mathrm{C}$. To check for potential sampling bias, we took advantage of a rare feature - the information about the vertical position and species identity of all $>22,000$ individuals in the epiphyte community (Glenda Mendieta-Leiva \& Gerhard Zotz, unpublished data). We used this information in combination with intraspecific trait-height relationships (see H2) to additionally predict community trends when considering the entire community (for details see Fig. A.2). Qualitative comparisons with the community trends based on sampled individuals were used to detect sampling bias in observed trends.

To assess how trait syndromes (i.e. combinations of multiple traits of individuals) are influenced by their vertical position, we first conducted a principal component analysis (PCA; R package 'vegan') based on the normalized and centered trait data of the extensively sampled traits. Some leaf traits covaried strongly (pairwise correlations between all traits were assessed by Pearson's correlation coefficient), and the PCA reduced correlated traits to independent components. 


\section{Functional leaf traits of vascular epiphytes}

Subsequently, LMs using the PCA scores of the first two PCA axes as dependent variables and height as independent variable were applied.

\section{H2 - Variations in trait-height relationships among species influence community trait structure}

We analyzed the influence of variations in trait-height relationships among species on community trait structure by comparing LMs with different fixed effects (trait fixed effects: height x species, height + species, height, species) based on AIC values. Simpler LMs with fewer fixed effects and no interactions were selected as MAM when $\triangle \mathrm{AIC} \leq 10$ (Burnham \& Anderson 2004). When the MAM included the interaction of height and species, the community trait structure was significantly influenced by differences in mean trait values and differences in trait responses to height among species. If the MAM included species as fixed effect but no interaction, only differences in mean trait values among species were significant.

Additionally, to assess the importance of intraspecific trait responses for each trait, we classified species based on the significance of their trait-height relationship (non-significant slopes, significant positive, or negative slopes; $P<0.05$ ). We only used species with $\geq 10$ records per trait for these analyses $(\mathrm{n}=51)$, which excluded the $\mathrm{NC}$ traits.

\section{H3 - Vertical ranges of epiphyte species correlate with their intraspecific trait variability}

To assess the general importance of intraspecific trait variability, we first carried out variance component analyses (R package 'varcomp'), which partition observed trait variability into withinspecies (intraspecific) and between-species (interspecific) components (Messier, McGill \& Lechowicz 2010). Subsequently, we calculated two measures of trait variability for each species: the coefficient of variation (CV) and the trait range (TR: absolute difference between maximum and minimum trait value divided by the maximum, given in \%). The relationship between these measures of intraspecific trait variability and species' vertical ranges was analyzed with LMs (vertical range $\sim$ trait variability), whereby the vertical range for each species was estimated based on its maximum and minimum height observed in the census. 
H4 - Trait means, trait syndromes and trait-height relationships differ among taxonomic groups

Differences in trait means among the major taxonomic groups (aroids, bromeliads, orchids, ferns; Table 2.1), based on trait means of associated species, were compared using max- $t$ tests for multiple comparisons that account for unbalanced group sizes, non-normality and heteroscedasticity (R packages 'multcomp' and 'sandwich'; see Herberich, Sikorski \& Hothorn 2010).

Differences in trait syndromes among the taxonomic groups were tested using a permutational multivariate analysis of variance (PERMANOVA, adonis from 'vegan' R package; Anderson 2001). Additionally, we used the PCA results to visualize differences among taxonomic groups.

Differences in trait-height relationships among the taxonomic groups were analyzed using generalized linear mixed models (see Fig. A.3 and Table A.2 for details).

\subsection{Results}

\section{H1 - Trait means and trait syndromes change with height at the community level}

All leaf traits were significantly correlated with height $(P<0.05$, Fig. 2.1 , Table A.3). The strongest correlations between community trait means (for 1-m height intervals) and height were observed for SLA $\left(R^{2}=0.89\right), C h l_{\text {mass }}\left(R^{2}=0.76\right)$, leaf thickness $\left(R^{2}=0.72\right), \delta^{13} C\left(R^{2}=0.66\right)$ and $\mathrm{LWC}_{\text {area }}\left(\mathrm{R}^{2}=0.64\right)$. Trait-height correlations were generally much weaker when, instead of community means, traits of all sampled individuals were used as response variable: in this case only $\delta^{13} \mathrm{C}\left(\mathrm{R}^{2}=0.35\right)$, SLA $\left(\mathrm{R}^{2}=0.30\right)$ and $\mathrm{Ch} l_{\text {mass }}\left(\mathrm{R}^{2}=0.16\right)$ were moderately correlated with height (Fig. 2.1). While SLA, $\mathrm{Chl}_{\text {mass }}$ and $\mathrm{N}_{\text {mass }}$ showed decreasing, non-linear trends with height, leaf thickness, $\mathrm{LWC}_{\text {area }}$ and $\delta^{13} \mathrm{C}$ showed positive linear trends with height (Fig. 2.1). LDMC and $\delta^{15} \mathrm{~N}$ showed slightly negative trends, but rather weak correlations. Observed community trends were largely consistent with those considering the entire censused community, indicating no substantial sampling bias (compare Figs. 2.1 and A.2, as well as Table A.3 and A.4).

Many traits covaried significantly (Table A.5; $P<0.05$ ), for instance leaf thickness and $\mathrm{LWC}_{\text {area }}$ $(\mathrm{r}=0.84), \mathrm{Ch}_{\text {mass }}$ and $\mathrm{N}_{\text {mass }}(\mathrm{r}=0.67)$, as well as SLA and $\mathrm{Ch}_{\text {mass }}(\mathrm{r}=0.64)$. The first two PCA axes explained $45 \%$ and $25 \%$, respectively, of variation in leaf traits. Height explained $16 \%$ of variation along the first axis and $7 \%$ along the second axis $(P<0.001)$. 

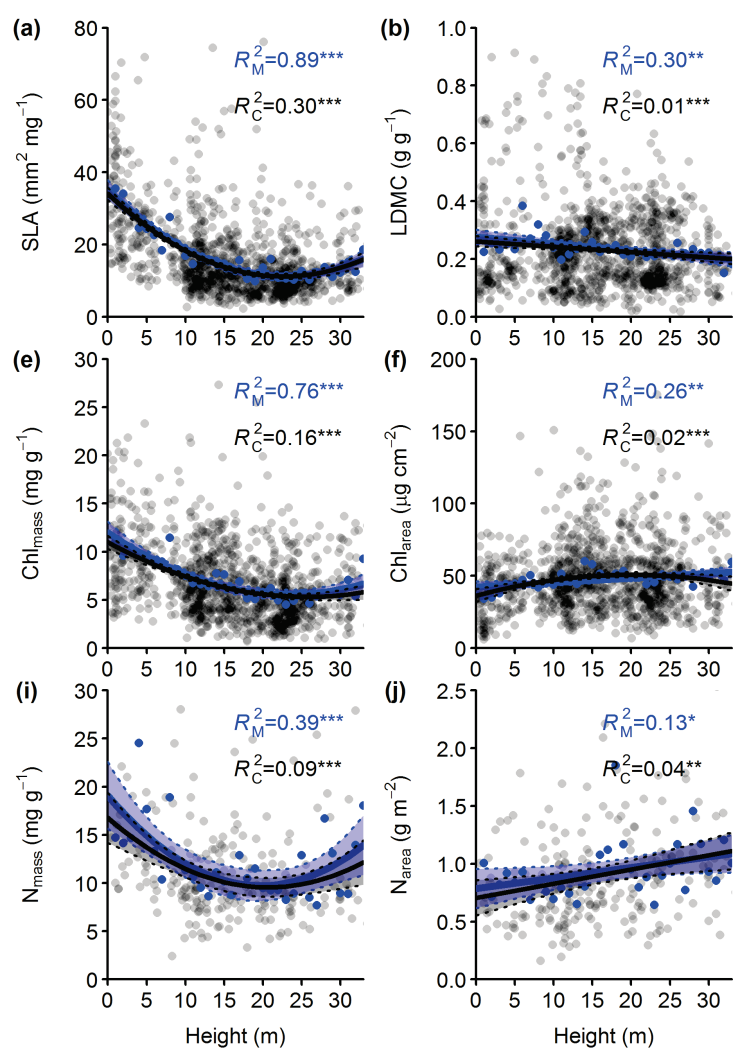

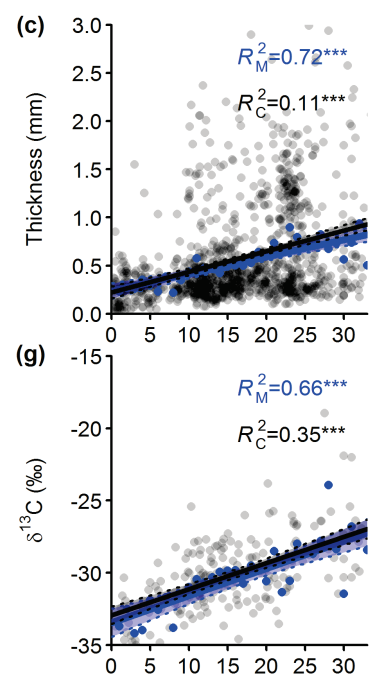

Height $(m)$

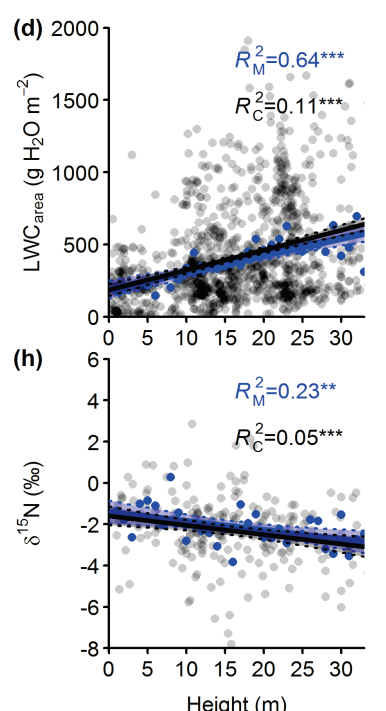

- Community mean trait values

for 1-m height intervals

and regression line

- Trait values of individuals and regression line

Figure 2.1. Trait-height relationships of vascular epiphytes for ten leaf traits: (a) SLA: specific leaf area, (b) LDMC: leaf dry matter content, (c) Thickness: leaf thickness, (d) $\mathrm{LWC}_{\text {area }}$ : leaf water content per leaf area, (e) Chl mass: massbased chlorophyll concentration, (f) Chlarea: area-based chlorophyll concentration, (g) $\delta^{13} \mathrm{C}$ : carbon isotope ratio, (h) $\delta^{15} \mathrm{~N}$ : nitrogen isotope ratio, (i) $\mathrm{N}_{\text {mass }}$ : mass-based nitrogen concentration, (j) $\mathrm{N}_{\text {area }}$ : area-based nitrogen concentration. Simple LMs (trait $\sim$ height) and LMs including a quadratic term (trait $\sim$ height + height ${ }^{2}$ ) were fitted and compared by AIC. Non-linear models were preferred when $\triangle \mathrm{AIC} \leq 10$ (Table $\mathrm{S} 3$ for summary statistics). $\mathrm{R}^{2} \mathrm{M}$ : amount of variance in community means explained by height. $\mathrm{R}^{2} \mathrm{C}$ : amount of variance in individuals' trait values explained by height. Asterisks indicate significance levels of trait-height relationships (*** $P<0.001,{ }^{* *} P<0.01, * P<0.05$ ). Shaded areas indicate $95 \% \mathrm{CI}$.

\section{H2 - Variations in trait-height relationships among species influence community trait} structure

The MAM for SLA, LDMC, leaf thickness and $\mathrm{Chl}_{\text {mass }}$ included the interaction between species and height, indicating that the community structure for these traits was best explained when considering that species differ in both their trait means and their trait responses to height (Table A.6. In contrast, for $\mathrm{Chl}_{\text {area }}$ and $\mathrm{LWC}_{\text {area }}$ only between-species differences in trait means were significant (Table A.6).

Seventy percent of all species had at least one trait that was significantly correlated with height. Significant intraspecific trait-height relationships $(P<0.05)$ were most common for SLA, for which $45 \%$ of all species revealed a significant relationship with height, followed by LDMC with 33\% (Table A.7; see Figs. A.4-A.9 for intraspecific trait-height relationships of all species). The directions of intraspecific trait-height relationships were largely consistent within traits. For 
instance, for SLA and $\mathrm{Chl}_{\text {mass, }}$, slopes were invariably negative (Table A.7). However, for LDMC and thickness, there were a few species showing opposing trends (Table A.7).

\section{H3 - Vertical ranges of epiphyte species correlate with their intraspecific trait variability}

Variance component analysis revealed that intraspecific variability, on average, accounted for $31 \%$ of observed variance (Fig. A.10). The proportion of variance explained by intraspecific variability ranged from $16 \%$ (Thickness) to $51 \%$ (Chl ${ }_{\text {area }}$ ).

We observed significant positive correlations between both measures of multivariate intraspecific trait variability (mean CV, mean TR) and vertical ranges of species (Fig. A.11). The correlation was stronger for mean TR $\left(\mathrm{R}^{2}=0.24, P<0.001\right)$ than for mean $\mathrm{CV}\left(\mathrm{R}^{2}=0.10, P=0.009\right)$.

\section{H4 - Trait means, trait syndromes and trait-height relationships differ among taxonomic groups}

We found significant differences between trait means of taxonomic groups for all traits except $\mathrm{N}_{\text {area }}, \delta^{13} \mathrm{C}$ and $\delta^{15} \mathrm{~N}$ (Table 2.1). Trait differences were, however, often only significant between individual taxonomic groups; there was no trait for which all pairwise differences were significant. The only case in which a group's trait mean differed significantly from that of all other groups was LDMC, with almost two-fold higher values in ferns (Table 2.1). Orchids had, on average, the thickest leaves, the highest $\mathrm{LWC}_{\text {area }}$ and the smallest SLA, but differences in these traits were consistently significant only compared to ferns (Table 2.1). Bromeliads tended towards low nitrogen and chlorophyll concentrations, although differences were not always significant. In contrast, the highest nitrogen and chlorophyll concentrations were consistently found in aroids.

Taxonomic groups also differed significantly in height distributions. The mean height of orchid species $(21.0 \pm 6.4 \mathrm{~m})$ was significantly higher $(P<0.05$, max- $t$ test $)$ than that of aroids $(12.2 \pm 7.5$ $\mathrm{m})$ and ferns $(11.1 \pm 7.2 \mathrm{~m})$, but did not differ significantly from that of bromeliads $(14.7 \pm 7.4 \mathrm{~m}$; Table 1).

The PERMANOVA indicated significant differences in trait syndromes among all taxonomic groups $(P<0.001)$. The dispersion of species in PCA trait space showed that several species of different taxa shared similar trait syndromes, but also that there were unique tendencies within taxonomic groups (compare, e.g., orchids and ferns; Fig. 2.2).

The fixed-effect structure of the MAMs did not include the interaction between height and taxonomic group for any leaf trait, indicating that slopes of trait-height relationships did not differ significantly among taxonomic groups (Table A.2). No significant differences in slopes or intercepts were observed for SLA and all NC traits (Fig. A.3). 
Table 2.1. Mean leaf trait values \pm SD of the major taxonomic groups of vascular epiphytes (aroids, bromeliads, orchids, ferns) in a Panamanian lowland forest. Species from all other taxa are summarized in 'Others'. CAM species were excluded from $\delta^{13} \mathrm{C}$ analyses. Differences between taxonomic groups were analyzed using max- $t$ tests for multiple comparisons of means, and significant differences in trait means $(P<0.05)$ are indicated by different letters. Proportions of sampled individuals and species are given in parentheses. ${ }^{*}$ For the NC traits, not all species were sampled, sample sizes were: Aroids: $n=10$, Bromeliads: $n=5$, Orchids: $n=19-24$, Ferns: $n=17$, Others: $n=5$.

\begin{tabular}{|c|c|c|c|c|c|}
\hline & Aroids & Bromeliads & Orchids & Ferns & Others \\
\hline Individuals & $149(12.9 \%)$ & $62(5.4 \%)$ & $435(37.8 \%)$ & $379(32.9 \%)$ & $126(10.9 \%)$ \\
\hline Species & $13(15.7 \%)$ & $5(6 \%)$ & $32(38.6 \%)$ & $24(28.9 \%)$ & $9(10.8 \%)$ \\
\hline Height (m) & $12.2 \pm 7.5^{\mathrm{A}}$ & $14.7 \pm 7.4^{\mathrm{AB}}$ & $21.0 \pm 6.4^{\mathrm{B}}$ & $11.1 \pm 7.2^{\mathrm{A}}$ & $15.4 \pm 3.0^{\mathrm{A}}$ \\
\hline $\operatorname{SLA}\left(\mathrm{mm}^{2} \mathrm{mg}^{-1}\right)$ & $22.1 \pm 10.9^{\mathrm{AB}}$ & $17.7 \pm 7.5^{\mathrm{AB}}$ & $14.0 \pm 5.7^{\mathrm{A}}$ & $24.2 \pm 14.8^{\mathrm{B}}$ & $27.6 \pm 11.2^{\mathrm{B}}$ \\
\hline $\operatorname{LDMC}\left(\mathrm{g} \mathrm{g}^{-1}\right)$ & $0.17 \pm 0.05^{\mathrm{A}}$ & $0.18 \pm 0.04^{\mathrm{A}}$ & $0.20 \pm 0.10^{\mathrm{A}}$ & $0.34 \pm 0.11^{\mathrm{B}}$ & $0.08 \pm 0.05^{\mathrm{C}}$ \\
\hline Thickness (mm) & $0.38 \pm 0.13^{\mathrm{A}}$ & $0.59 \pm 0.69^{\mathrm{AB}}$ & $0.70 \pm 0.52^{\mathrm{B}}$ & $0.26 \pm 0.12^{\mathrm{A}}$ & $0.96 \pm 0.59^{\mathrm{B}}$ \\
\hline $\mathrm{LWC}_{\text {area }}\left(\mathrm{g} \mathrm{H}_{2} \mathrm{O} \mathrm{m}^{-2}\right)$ & $297 \pm 102^{\mathrm{A}}$ & $378 \pm 278^{\mathrm{ABC}}$ & $488 \pm 344^{\mathrm{B}}$ & $168 \pm 128^{C}$ & $632 \pm 325^{\mathrm{B}}$ \\
\hline $\mathrm{Chl}_{\text {mass }}\left(\mathrm{mg} \mathrm{g}^{-1}\right)$ & $10.1 \pm 2.9^{\mathrm{A}}$ & $5.4 \pm 3.2^{\mathrm{AB}}$ & $6.3 \pm 2.6^{\mathrm{B}}$ & $7.5 \pm 2.7^{\mathrm{AB}}$ & $8.3 \pm 3.9^{\mathrm{AB}}$ \\
\hline $\mathrm{Chl}_{\text {area }}\left(\mu \mathrm{g} \mathrm{cm}^{-2}\right)$ & $55.3 \pm 16.4^{\mathrm{A}}$ & $31.3 \pm 9.1^{\mathrm{B}}$ & $47.6 \pm 15.3^{\mathrm{A}}$ & $41.4 \pm 19.1^{\mathrm{AB}}$ & $32.3 \pm 9.6^{\mathrm{B}}$ \\
\hline$\delta^{13} \mathrm{C}(\%) *$ & $-29.7 \pm 2.7^{\mathrm{A}}$ & $-29.9 \pm 0.7^{\mathrm{A}}$ & $-29.7 \pm 2.1^{\mathrm{A}}$ & $-31.2 \pm 1.6^{\mathrm{A}}$ & $-30.4 \pm 2.0^{\mathrm{A}}$ \\
\hline$\delta^{15} \mathrm{~N}(\% o)^{*}$ & $-1.8 \pm 1.9^{\mathrm{AB}}$ & $-2.8 \pm 1.0^{\mathrm{AB}}$ & $-2.3 \pm 1.0^{\mathrm{AB}}$ & $-1.7 \pm 1.1^{\mathrm{A}}$ & $-3.8 \pm 1.1^{\mathrm{B}}$ \\
\hline $\mathrm{N}_{\text {mass }}\left(\mathrm{mg} \mathrm{g}^{-1}\right)^{*}$ & $14.5 \pm 6.4^{\mathrm{A}}$ & $7.5 \pm 2.1^{\mathrm{B}}$ & $11.6 \pm 5.5^{\mathrm{AB}}$ & $12.0 \pm 3.6^{\mathrm{A}}$ & $11.8 \pm 4.0^{\mathrm{AB}}$ \\
\hline $\mathrm{N}_{\text {area }}\left(\mathrm{g} \mathrm{m}^{-2}\right)^{*}$ & $1.07 \pm 0.29^{\mathrm{A}}$ & $0.57 \pm 0.36^{\mathrm{A}}$ & $0.92 \pm 0.33^{\mathrm{A}}$ & $0.96 \pm 0.38^{\mathrm{A}}$ & $0.69 \pm 0.29^{\mathrm{A}}$ \\
\hline
\end{tabular}




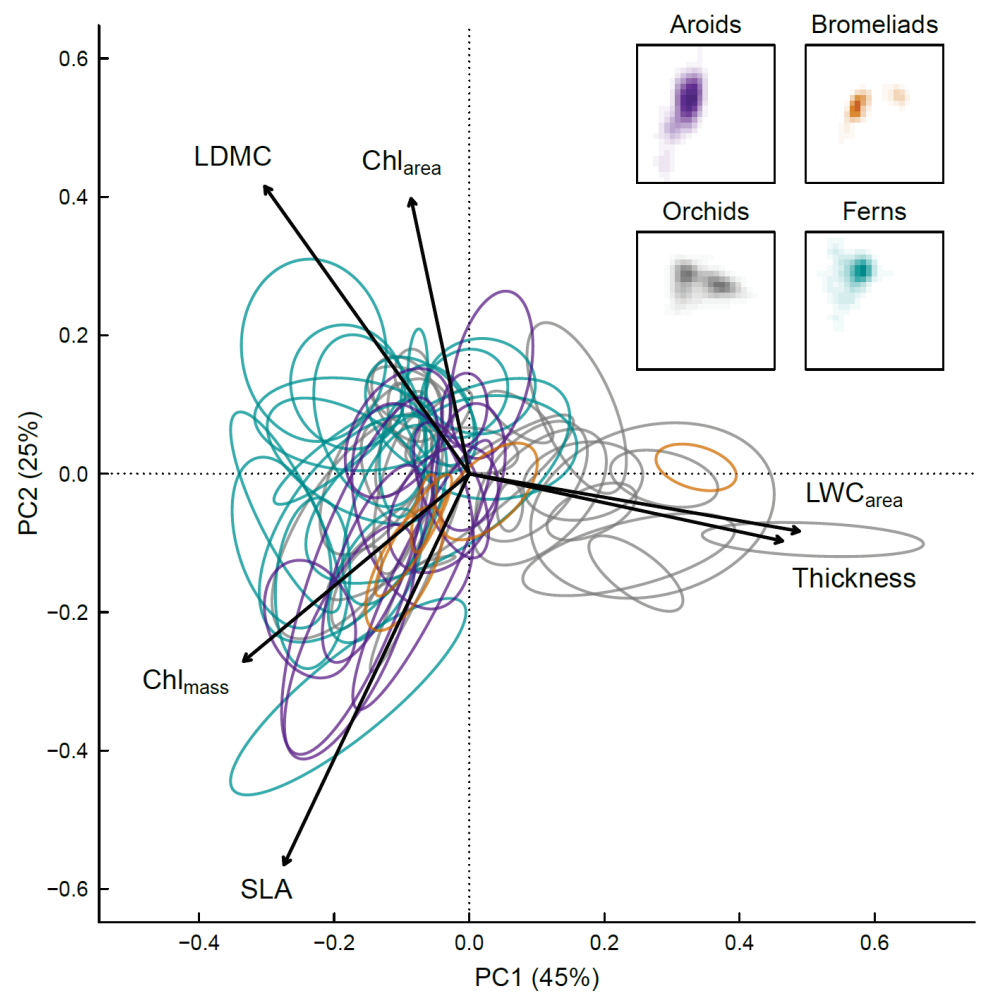

Figure 2.2. Dispersion of trait syndromes of epiphyte species in the PCA trait space based on six leaf traits of 1,151 individuals. Trait syndromes of all species belonging to four major taxonomic groups (aroids, bromeliads, orchids, ferns) are shown as ellipsoids of inertia, which encompass $95 \%$ of individuals of each species. The position and the spread of the ellipsoids thus illustrate mean trait syndromes and trait variability of each species. Insets in the upper right part show kernel densities for each taxonomic group. A PERMANOVA indicated significant differences in trait syndromes between all taxonomic groups $(P<0.001)$.

\subsection{Discussion}

\section{H1 - Trait means and trait syndromes change with height at the community level}

Our results support the hypothesis that community trait means of vascular epiphytes are significantly correlated with height, but strength and direction of correlations varied considerably. The strongest correlations among the extensively sampled traits were found for SLA and Chl mass, whose negative trends from the forest floor to the upper canopy are consistent with differences between sun and shade leaves of tropical trees (Rozendaal et al. 2006; Markesteijn et al. 2007) and trends along tree height gradients (Rijkers, Pons \& Bongers 2000). When considering that SLA and $\mathrm{Chl}_{\text {mass }}$ covaried considerably and that $\mathrm{Ch}_{\text {area }}$ did not show a strong vertical trend, it seems likely that changes in $\mathrm{Ch}_{\text {mass }}$ were mainly driven by changes in $\mathrm{SLA}\left(\mathrm{Ch} l_{\text {mass }}=\mathrm{Chl}_{\text {area }}\right.$ SLA). In soil rooted plants, vertical gradients in SLA are commonly related to vertical light gradients (Poorter 1999; McMurtrie \& Dewar 2011) but hydraulic constraints have also been discussed (Rijkers, Pons \& Bongers 2000; Koch et al. 2004). A comparative study by Cavaleri et al. (2010) found that epiphytes were the only plant group for which light was most important in explaining vertical SLA profiles, which seems logical as epiphytes lack a hydraulic connection to 


\section{Functional leaf traits of vascular epiphytes}

the ground. Because SLA relates the light-capturing leaf area to investment in dry mass, an increase in SLA increases the potential carbon gain per biomass investment. However, increased light-capture efficiency via high SLA tends to be associated with higher respiration rates and shorter leaf lifespans. Several such correlations between leaf traits capturing fundamental aspects of leaf economics have been observed ('worldwide leaf economics spectrum'; Wright et al. 2004). Theoretical models have demonstrated that, when considering these between-trait correlations, the carbon gain over the leaf lifespan is maximized when SLA increases non-linearly with decreasing light (Sims, Gebauer \& Pearcy 1994; McMurtrie \& Dewar 2011). The non-linearly decreasing community means of SLA with height (Fig. 2.1a) agree with these expectations and corroborate the notion that light is the main driver of vertical SLA profiles in epiphytes.

The observed increase in leaf thickness with height is consistent with within-individual, intra- and interspecific vertical trends found in trees (Rozendaal et al. 2006; Markesteijn et al. 2007). Apart from maximization of carbon gain, avoidance of damages and water loss minimization are also requirements of optimal leaf functioning: an increase in leaf thickness is regarded as adjustment to prevent overheating and to balance carbon gain and transpiration water-loss under drier and sunnier conditions (Cornelissen et al. 2003; Rozendaal et al. 2006). Without anatomical adjustments changing leaf tissue density, a decrease in SLA would induce an increase in leaf thickness, which partially explains their covariance $(r=-0.48)$. Nevertheless, the linear increase in leaf thickness (Fig. 2.1c) in contrast to the non-linear decrease in SLA (Fig. 2.1a) suggests that the trend in leaf thickness is not only related to SLA, but also to independent morphological adjustments which are probably more influenced by the vertical gradient in potential evapotranspiration than by the vertical light gradient.

In general, LDMC also tends to scale with SLA and is sometimes regarded as an alternative predictor of plant strategies (Wilson, Thompson \& Hodgson 1999). Interestingly, the observed covariance between LDMC and SLA was rather low at community level ( $\mathrm{r}=-0.16)$ and the LDMCheight correlation was rather weak (Fig. 2.1b). This suggests that plant functioning captured by SLA is more relevant along vertical gradients within forests.

It is well established that the proportion of epiphytes with CAM increases with height (e.g. Zotz 2004). The positive trend in $\delta^{13} \mathrm{C}_{\text {of }} \mathrm{C}_{3}$ plants documented here (Fig. 2.1g) has arguably the same ecological background: more demanding water relations result in increasing stomatal limitations (Farquhar, Ehleringer \& Hubick 1989). Tissue $\delta^{13} \mathrm{C}$ correlates with water-use efficiency, and $\delta^{13} \mathrm{C}$ is thus used as indicator of water-stress. However, along vertical gradients in forests, interpretation may be confounded as the atmospheric $\delta^{13} \mathrm{C}$ signature also shows a vertical trend (Quay, King \& Wilbur 1989). Nevertheless, the strongest increase in atmospheric $\delta^{13} \mathrm{C}$ signature occurs within a few meters above the forest floor due to soil respiration, and above this zone, the gradient in $\delta^{13} \mathrm{C}$ is generally weak (Quay et al. 1989). In contrast, our model predicted a linear 
trend with an average change of $\sim 5.5 \%$ in $\delta^{13} \mathrm{C}$ from the trunk base to the upper canopy (Fig. $2.1 \mathrm{~g}$ ), suggesting that a large part of the observed variance in tissue $\delta^{13} \mathrm{C}$ can be attributed to differences in water-use efficiency. These results agree with observations for leaves of tropical trees (Medina \& Minchin 1980). In contrast, difference in $\delta^{13} \mathrm{C}$ signals of epiphytes between the upper and lower parts of a lowland rainforest were smaller ( $<2 \%$; Wania et al. 2002), possibly due to a less pronounced gradient of water stress than in our system (precipitation at this site in Costa Rica is $>6000 \mathrm{~mm} /$ year). Alternatively, the discrepancy may indicate that height above ground is a better predictor for water stress than the predefined Johansson zones used by Wania et al. (2002), which subdivide host trees according to their principal structure without considering absolute height.

In line with Wania et al. (2002), we observed a negative trend in tissue $\delta^{15} \mathrm{~N}$ with height (Fig. 2.1h). The $\delta^{15} \mathrm{~N}$ signatures of plants are mostly affected by their assimilatory pathway, but also by form $\left(\mathrm{NO}_{4}{ }^{+}, \mathrm{NH}_{3}{ }^{-}, \mathrm{N}_{2}\right)$ and $\delta^{15} \mathrm{~N}$ signature of the nitrogen source (Evans 2001). Epiphytes use a blend of different autochthonous (e.g. canopy soil, leachates) and allochthonous nitrogen sources (e.g. wet and dry deposition), which can vary substantially in $\delta^{15} \mathrm{~N}$ signatures (Wania et al. 2002). The observed negative trend with height indicates an increasing contribution of atmospheric $\mathrm{N}$ to epiphyte $\mathrm{N}$ in the upper canopy. However, as we did not measure source $\delta^{15} \mathrm{~N}$ signatures, caution is needed when interpreting tissue $\delta^{15} \mathrm{~N}$ trends.

In summary, we found only moderate to weak correlations between leaf traits/leaf trait syndromes and height when considering all individuals, but often strong correlations between community means and height. This also reflects that height is a suitable proxy of general vertical trends in environmental conditions, although it does not capture all relevant factors and small-scale environmental variability (Fig. A.1).

\section{H2 - Variations in trait-height relationships among species influence community trait structure}

For four out of six traits, the community trait structure could be best explained when including differences in intraspecific trait response to height, which supports our hypothesis for most traits. Intraspecific trait responses to height were particularly important for SLA, which was the trait with the highest frequency of significant trait-height relationships (45\% of all species) and consistently showed only negative trends. SLA captures essentials of leaf economics (Wright $e t$ al. 2004) and is a suitable trait for intraspecific adjustments because it can be relatively easily adjusted by varying size, number and cell wall thickness of different leaf cell types (Shipley et al. 2006; Kichenin et al. 2013). In general, although we cannot rule out genetic variation as source of intraspecific trait variability, we argue that, considering the spatial scale in our study, 


\section{Functional leaf traits of vascular epiphytes}

phenotypic trait plasticity in response to the environment is probably more important (also see Grassein, Till-Bottraud \& Lavorel 2010).

Interestingly, the second most frequent significant intraspecific trait-height relationships were found for LDMC, which, in contrast, was rather weakly correlated with height at the community level. The high frequency might partly be explained by correlations between SLA and LDMC, which can be much stronger at the species level than at the community level (compare Figs. A.4 and A.5). However, species-specific differences in strategies might also play a role (Wilson et al. 1999). For instance, in Elaphoglossum doanense only LDMC was strongly correlated with height $\left(\mathrm{R}^{2}=0.81\right)$ while there was no significant correlation for any of the other traits.

Although intraspecific trait response to height was common in epiphytes, the absence of a significant intraspecific trait-height relationship was not always accompanied by limited trait variability. Most species that lacked a significant trait-height correlation had a pronounced intraspecific trait variability unrelated to height. Apart from the uncertainties associated with height as proxy for environmental gradients, plant size and age are additional sources of intraspecific trait variability (Zotz 2000; Wanek et al. 2002; Hietz \& Wanek 2003), which might weaken trait-height relationships. It is therefore striking that height emerged as significant factor for intraspecific changes in leaf traits.

In summary, our results corroborate the growing evidence that not only differences in trait means, but also differences in intraspecific trait response to environmental gradients among species are non-negligible aspects of community assembly (Bolnick et al. 2011; Kichenin et al. 2013).

\section{H3 - Vertical ranges of epiphyte species correlate with their intraspecific trait variability}

Intraspecific variability explained almost one third of the observed variance in our trait data, which is in the same range as observed for terrestrial plants (Hulshof \& Swenson 2010; Albert et al. 2010b). This supports previous findings underlining the importance of considering trait variability not only between but also within species (Albert et al. 2010b). Such intraspecific variability seems to be important for species' spatial distribution, as our results supported the hypothesis that species occupying larger vertical ranges tended towards higher leaf trait variability (Fig. A.11). The inherent ability of species to vary their leaf traits might increase their ability to tolerate a wider range of environmental conditions (van Valen 1965). In this context, it is not surprising that TR explained a larger amount of variation in species vertical ranges than CV (TR: $\mathrm{R}^{2}=0.24, \mathrm{CV}: \mathrm{R}^{2}=0.10$ ). This is because $\mathrm{TR}$ is based on extreme trait values and is thus a better approximation of the theoretical maximal trait range of a species, whereas $\mathrm{CV}$ is affected by the trait frequency distribution. Sides et al. (2014) conducted a comparable study of 21 herbaceous perennials along an elevational gradient of ca. $700 \mathrm{~m}$, using $\mathrm{CV}$ as measure of trait variability. 
They observed a stronger correlation between intraspecific trait variability in SLA and elevational range $\left(\mathrm{R}^{2}=0.51\right)$. The weaker correlation in our study might partly be explained by the uncertainties associated with the height gradient as approximation of environmental gradients. Furthermore, Sides et al. (2014) pointed out that intraspecific trait plasticity should be essential when strong trends in community mean trait values exist. Community mean trends were less pronounced in our study, indicating that height was a weaker filter than elevation. In summary, epiphyte species that can adjust their leaf traits to the environment can potentially occupy larger vertical ranges. However, the substantial amount of unexplained variance also emphasizes that unstudied characteristics (e.g. root traits, specific morphological and physiological characteristics) or other processes (e.g. germination, seedling survival) might be likewise important in explaining why some species are restricted to smaller vertical ranges than others.

\section{H4 - Trait means, trait syndromes and trait-height relationships differ among taxonomic groups}

For most traits, we found significant differences in trait means between taxonomic groups, which partially confirm our hypothesis. Trait differences were, however, often only significant between certain pairs of taxonomic groups. The frequent absence of pairwise differences was mainly due to the high trait variation between species within taxonomic groups, and less due to similarities in group trait means. The pronounced within-group trait variation and associated among-group trait overlap become apparent when comparing species' trait syndromes in the multivariate trait space (Fig. 2.2). Nevertheless, the unique tendencies within taxonomic groups indicate that some leaf traits are taxonomically conserved (Fig. 2.2).

The marked differences in morphological leaf traits between orchids and ferns were consistent with previous studies reporting orchids having thicker leaves and lower SLA (Stuntz \& Zotz 2001; Cardelús \& Mack 2010). Community means of leaf thickness and SLA were strongly correlated with height, which emphasizes their functional relevance along the vertical gradient. It is thus unsurprising that differences in these traits were reflected in different height distributions of these taxa (Table 2.1; also see Fig. 2.3). This pattern might be partly explained by environmental filtering of species with unsuitable traits, but intraspecific leaf trait adjustments, particularly for SLA, might also be important. Interestingly, SLA was the only extensively sampled trait without significant differences in slopes or intercepts among the taxonomic groups (Fig. A.3a). This suggests an optimal SLA value at a given height independent of taxonomic group and further indicates that environmental changes along the height gradient act as a particularly strong filter on SLA. 


\section{Functional leaf traits of vascular epiphytes}

The most striking among-group differences were observed for LDMC, with LDMC of ferns being twofold higher, on average, than in all other groups. LDMC values have not been reported for many epiphyte species, but Woods (2013) also found high LDMC values in two Elaphoglossum species and low values in one Microgramma species. This agrees with our results and shows that the LDMC of fern species can differ substantially (Table A.1). However, the large number of fern species sampled in our study $(n=24)$ suggests that high LDMC values are more common in ferns. Aroids had the highest leaf nitrogen and chlorophyll concentrations, whereas bromeliads consistently had the lowest. In fact, both traits were correlated ( $\mathrm{r}=0.67$; Table S5). Chlorophyll concentrations have not yet been compared among major epiphyte taxa, but our results agree with reported leaf nitrogen values. For example, Stuntz \& Zotz (2001) also found the highest nitrogen concentrations in aroids. Lowest nitrogen concentrations, in turn, were consistently observed in bromeliads (Hietz, Wanek \& Popp 1999; Stuntz \& Zotz 2001; Cardelús \& Mack 2010). An increase in leaf nitrogen content is usually associated with an increase in photosynthetic capacity (Stuntz \& Zotz 2001; Wright et al. 2004). Interestingly, differences in photosynthetic nitrogenuse efficiency (PNUE) were observed among epiphyte taxa, with aroids having the lowest PNUE, and bromeliads having the highest (Stuntz \& Zotz 2001). Thus, for a given nitrogen concentration, the photosynthetic capacity was higher in bromeliads. This suggests that the observed amongtaxa differences in leaf nitrogen cannot be used to infer similar differences in photosynthetic capacity.

We did not observe significant among-group differences in $\delta^{13} \mathrm{C}$ and $\delta^{15} \mathrm{~N}$ values. Our results largely agree with observations along an elevational gradient in Costa Rica (Cardelús \& Mack 2010). In contrast, Hietz et al. (1999) observed significantly depleted $\delta^{15} \mathrm{~N}$ values in bromeliads, but these were mainly of atmospheric habit. All these studies found high variability in isotope ratios of species within taxonomic groups, suggesting that the environmental conditions and species-specific characteristics are more important in determining isotope ratios in leaf tissue of individual epiphytes than their taxonomic affiliation.

Compared to global trait means of non-epiphytic taxa (TRY; Kattge et al. 2011), both low nitrogen concentrations and thick leaves are particularly noticeable differences (Table A.1 for details). These trait differences can be regarded as adaptation of epiphytes to an environment in which water and nutrients are only intermittently available. 


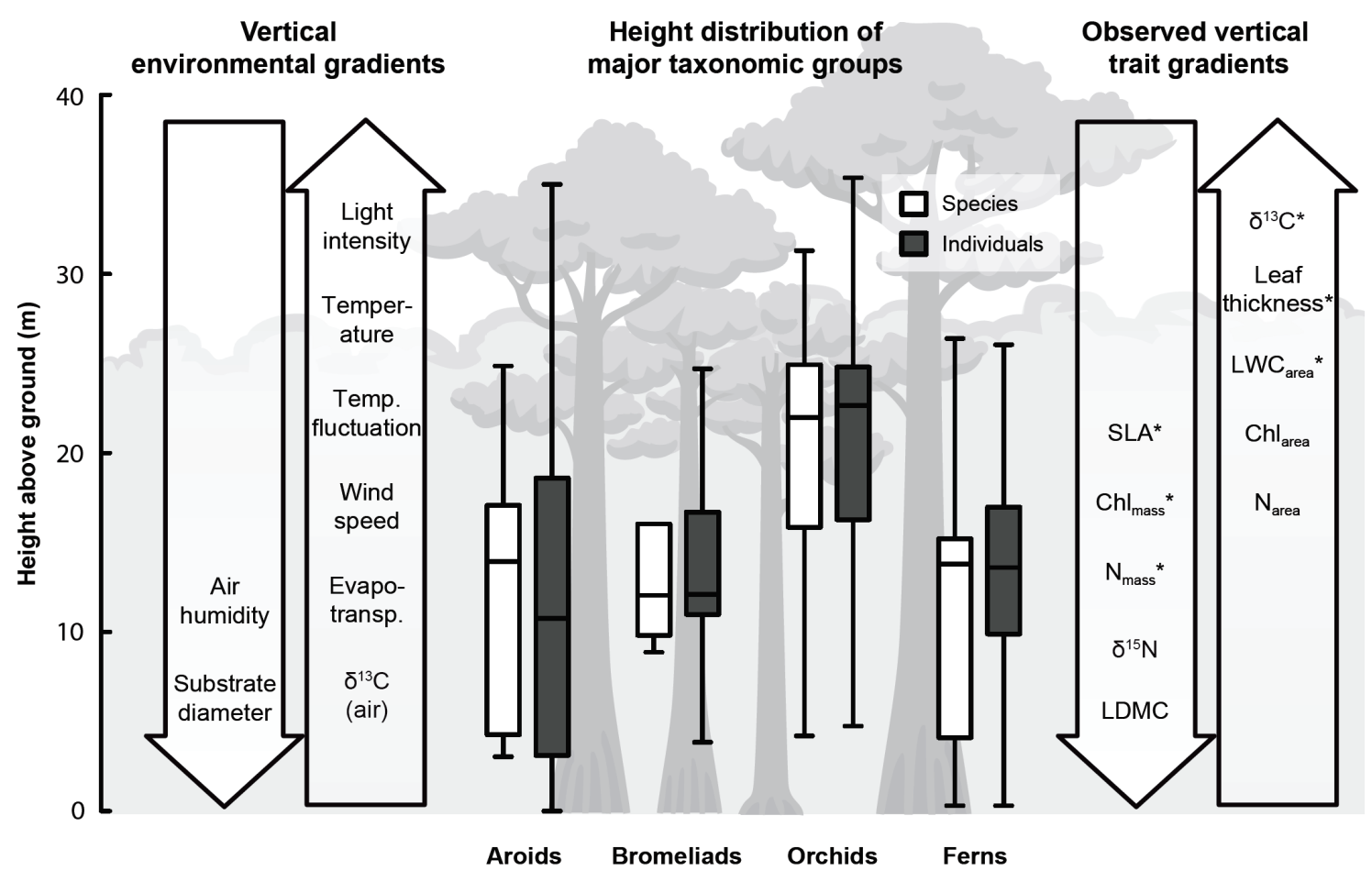

Figure 2.3. Schematic diagram illustrating main findings. Arrows on left side: Environmental factors commonly changing with height above ground within forests. In this study, only the vertical light gradient was measured (Fig. S1). Boxplots: Height distribution of the major taxonomic groups of epiphytes at the study site in Panama. Height distributions are based on either the height of each individual or the mean height of each species. Boxplots depict median heights (horizontal line), interquartile ranges (boxes), and approximate $95 \%$ confidence intervals (whiskers). Outliers are not shown. Arrows on right side: Significant vertical leaf trait gradients at the study site (trait abbreviations as in Fig. 1). Leaf traits showing pronounced changes in community trait means with height are marked by *.

\section{Conclusion}

Our findings indicate that analyzing multiple aspects of trait-based ecology (e.g. community and intraspecific trends, inter- and intraspecific variability, correlations among traits) is key to advance the understanding of mechanisms structuring plant communities. Leaf trait syndromes and intraspecific trait variability play an important role in explaining the vertical zonation of vascular epiphyte species and taxonomic groups (see Fig. 2.3 for a schematic representation of key findings). However, other adaptations of epiphytes, like water- and nutrient-storing pseudobulbs in orchids or phytotelmata in bromeliads, are probably likewise important. As height above ground as proxy of vertical environmental gradients explained substantial amounts of total trait variations, we propose to use height in addition to the more frequently used zonation scheme by Johansson in trait-based studies of epiphytes. 


\section{Acknowledgements}

We thank the Republic of Panama for making its natural resources available for science (research and export permits: SE/P-3-11 and SEX/P-27-11). Thanks to all the people of the Tropical Canopy Biology Program at STRI who made work at the crane possible. Fieldwork in Panama by GZ, KW and ES was funded by the DFG (Zo 94/5-1). GP and HK were funded by the DFG Initiative of Excellence Free Floater Program at the University of Göttingen. JSC acknowledges financial support by a German Research Foundation (DFG) grant (SA-21331).

\section{Data Accessibility}

Trait data have been uploaded to the Plant Trait Database TRY (http://www.try-db.org) and are available upon request. 


\section{Branchfall as a demographic filter for epiphyte communities: Lessons from forest floor-based sampling}

Juliano Sarmento Cabral, Gunnar Petter, Glenda Mendieta-Leiva, Katrin Wagner, Gerhard Zotz and Holger Kreft

published in PLoS One, 2015, 10, e0128019, DOI:10.1371/journal. pone.0128019 


\subsection{Abstract}

Local variation in abundance and richness of vascular epiphytes is often attributed to environmental (substrate and abiotic) characteristics. Less is known, however, about the impacts of tree and branch turnover on epiphyte communities. To address this issue, we surveyed branches and epiphytes found on the forest floor in a total of 96 transects in two forests (Atlantic rainforest in Brazil and Caribbean rainforest in Panama). In the Brazilian forest, we distinguished between edge and core. We quantified branch abundance, epiphyte abundance, richness and proportion of adults to investigate the trends of these variables over branch diameter. In the Panamanian forest, epiphytes had been previously inventoried, allowing an evaluation of our surveying method. Branches $<2 \mathrm{~cm}$ in diameter comprised $>90 \%$ of all branches on the forest floor. Abundance and richness of fallen epiphytes per transect were highest in the Brazilian core transects and lowest in the Panamanian transects. The majority of epiphytes on the floor (c. $65 \%$ ) were still attached to their branches. At all three study sites (Brazilian core, Brazilian edge and Panamanian transects), branch abundance and branch diameter were negatively correlated, whereas epiphyte abundance and richness per branch and proportion of adults were positively correlated with branch diameter. The relationship between branch diameter and absolute epiphyte abundance and richness differed between study sites, which might be explained by differences in forest structure and dynamics. Individuals found on the forest floor corresponded to $>12 \%$ of all individuals on branches $<10$ $\mathrm{cm}$ in diameter (including crowns), with abundance, richness and composition trends on forest floor reflecting canopy trends. We argue that forest floor surveys provide useful floristic and, most notably, demographic information particularly on epiphytes occurring on thinnest branches, which are the least accessible. Here, branchfall acts as an important demographic filter structuring epiphyte communities.

\subsection{Introduction}

Vascular epiphytes are plants that grow on shrubs and trees, and thereby on a substrate distributed in three-dimensional space (Benzing 1990; Zotz 2007; Krömer et al. 2007). Microclimatic conditions change dramatically within this three dimensional space, with generally drier and sunnier conditions towards the outer crowns of the trees (e.g. Woods, Cardelús \& Dewalt 2015). These conditions seem to cause higher drought-related mortality at early life stages compared to those in inner crowns and trunks (Wagner, Bogusch \& Zotz 2013). Moreover, abiotic conditions vary within inner-crowns and between tree species (Cardelús et al. 2005; Cardelús 2007). These environmental gradients have been suggested to structure the distribution of epiphyte species (Freiberg 1996; Freiberg \& Freiberg 2000; Zotz et al. 2005; Zotz 2007; Cardelús 2007; Woods et al. 2015). However, apart from the abiotic environmental conditions, the dynamics of the 


\section{Branchfall as demographic filter for epiphyte communities}

substrate itself should also influence epiphyte communities. This is because trees are constantly growing, producing new and losing older branches, meaning that the substrate persists only for a limited period (Malhi 2012; Woods et al. 2015). For example, trees commonly abscise branches lacking photosynthetically active leaves, which are more likely to be thin branches (Millington \& Chaney 1973; Addicott 1991). Moreover, thin branches stand less mechanical stress by epiphyte load, wind force, rainfall, or arboreal animals (Zotz et al. 2005). This might be particularly important in the outer crown of overstorey trees, but the crown of understory trees can also be disturbed by tree- and branchfall of large and emergent trees (Meer et al. 1996). Such a highly dynamic system should have profound consequences on the population and community dynamics of vascular epiphytes. In fact, because thin branches fall more often than thick branches, epiphytes growing on these thin branches are particularly susceptible to substrate failure (Hietz 1997). Correspondingly, only fast colonizing and maturing species are able to survive and reproduce on smaller branches (Chase 1987; Zotz 2007). Branchfall may thus profoundly influence distribution of epiphyte species within the canopy, contributing to niche partitioning in epiphyte communities.

Despite the apparent effects of diameter-dependent branchfall on community and population dynamics of epiphytes, related studies are rare. Hietz (1997) was able to quantify mortality rates via branchfall by monitoring selected branches through tree climbing and repeated photography. However, this technique is costly, requires training and is time-consuming. These limitations are a general barrier to improve our understanding on epiphyte ecology, as the accessibility of the tree canopies poses technical and logistic challenges. Among the several techniques that are currently used to assess the epiphytes, the use of binoculars is the simplest (Krömer et al. 2007; Werner \& Gradstein 2009), whereas tree climbing (Cardelús et al. 2006; Wolf, Gradstein \& Nadkarni 2009), tree climbing and photographs (e.g. Hietz 1997; Hietz, Ausserer \& Schindler 2002) and canopy cranes (Nieder et al. 2000; Zotz \& Schultz 2008) demand considerable work and/or investment efforts. An alternative, inexpensive method to gather information on epiphyte demography is to sample the forest floor, particularly if combined with data on branchfall, a main cause of epiphyte mortality (Hietz 1997). In fact, epiphytes on the forest floor may also provide information on the community structure and composition of epiphytes in the tree crowns, but this data is also surprisingly scarce in the literature (Mondragón \& Ticktin 2011). This is intriguing, given that epiphytes on the forest floor could be further assessed for sustainable economic activities, such as gathering of fallen individuals for horticulture (Mondragón \& Ticktin 2011; Toledo-Aceves, García-Franco \& López-Barrera 2014). Hence, despite the evident occurrence of epiphytes on the forest floor due to branchfall, this information source has been largely neglected.

To assess the usefulness of forest floor-based sampling to study vascular epiphytes, this study aimed to quantify branchfall and vascular epiphytes on the forest floor. This information was used to assess the relationship of epiphyte fall with branch diameter. For this purpose, we surveyed 
two Neotropical forests differing in epiphyte flora, elevation and climate (Atlantic submontane rainforest in northeastern Brazil and Caribbean lowland rainforest in Panama). We sampled edge and core forest habitats in the Brazilian forest. For each study site, we addressed three hypotheses for epiphytes on the forest floor: 1) epiphyte abundance, 2) epiphyte richness and 3) proportion of adult epiphytes are positively correlated with branch diameter (Fig. 3.1). In addition, we took advantage of data on exact three-dimensional positions of each individual epiphyte at the Panamanian site (Mendieta-Leiva, Wagner \& Zotz, unpubl. data) to evaluate our sampling method and results by assessing how patterns on the forest floor relate to the canopies. For this purpose, we compared epiphyte abundance, richness and composition of both forest floor and canopies. Overall, our results supported the hypotheses and demonstrated that branchfall-induced mortality has a non-negligible effect on the epiphyte community, particularly in the thin branches of tree canopies.

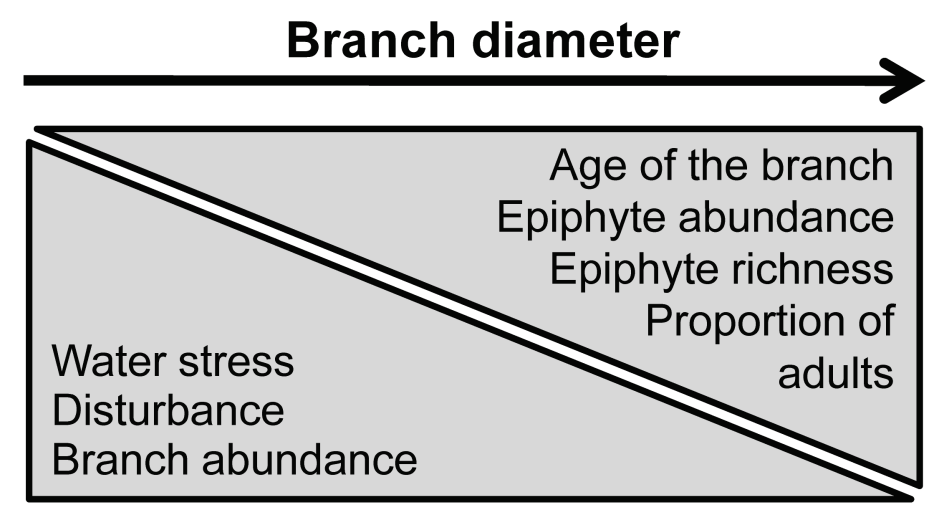

Figure 3.1. Schematic figure summarizing the expected trends with increasing branch diameter. We expect a decrease in water stress, mechanical disturbance and branch abundance with an increasing branch diameter. These drivers plus the increase in branch age should lead to higher epiphyte colonization and survival and an increase in epiphyte abundance, richness and proportion of adults per branch.

\subsection{Materials and methods}

\section{Study sites}

We surveyed branches and vascular epiphytes on the forest floor at two Neotropical forests. The first forest was located within Usina Serra Grande, a large private sugar-cane landholding in the State of Alagoas, northeastern Brazil ( $\left.8^{\circ} 58^{\prime} 50^{\prime \prime} \mathrm{S}, 35^{\circ} 54^{\prime} 30^{\prime \prime} \mathrm{W}\right)$. It is part of the fragmented Brazilian Atlantic forest (da Silva \& Tabarelli 2000) which retains c. $90 \mathrm{~km}^{2}$ of forest of the Pernambuco Centre of Endemism (Prance 1982), a unique biogeographic region within the Atlantic forest and a global biodiversity hotspot (Myers et al. 2000). We studied a forest fragment of c. 50 ha surrounded by a uniform matrix of sugar-cane monoculture. The forest fragment was 


\section{Branchfall as demographic filter for epiphyte communities}

located at c. $550 \mathrm{~m}$ a.s.1. in a fairly flat hilltop terrain, lacking gorges and riverbeds. The area receives c. $2000 \mathrm{~mm}$ of rainfall per year with a 3-month dry season $(<60 \mathrm{~mm} / \mathrm{month})$ from November to January and the wettest period is between April and August (Oliveira, Grillo \& Tabarelli 2004). The forest can be classified as lower montane or submontane rainforest. The fragment harbors a rich epiphyte flora (11 species of bromeliads, 2 cacti, 31 orchids and 4 peperomias; ferns and aroids have not been studied - (Siqueira Filho \& Felix 2005; Siqueira Filho et al. 2006). The fragment has a relatively old and stable edge ( $>80$ years), whose effects can be detected up to $100 \mathrm{~m}$ from the forest borders (Oliveira et al. 2004). We sampled both forest core and edge habitat (see next section). These two habitats are referred to as Brazilian core and Brazilian edge study sites.

The second forest and our third study site was the San Lorenzo Canopy Crane plot located near the Atlantic coast of the Republic of Panama $\left(9^{\circ} 16^{\prime} 50^{\prime \prime} \mathrm{N}, 79^{\circ} 58^{\prime} 30^{\prime \prime} \mathrm{W},[31]\right)$. The site is part of one of the largest undisturbed forest landscapes in Panama. It is at c. $130 \mathrm{~m}$ a.s.l. and receives c. $3500 \mathrm{~mm}$ of rainfall per year, with a three-month dry season $(<60 \mathrm{~mm} /$ month $)$ between January and March. The epiphyte flora has already been described in detail ( $>90$ species of holoepiphytes; Zotz \& Schultz 2008). At the crane site, only core forest conditions could be sampled, due to land mines from the US-American period of military training in the area outside the field station. The crane site is located in a narrow valley (slopes partially measuring slightly over $45^{\circ}$ of inclination), with a centrally-located and seasonally dry creek. The proximity to the Caribbean coast also exposes the area to frequent heavy storms and thus disturbances. The total area covered by the crane plot is c. 0.9 ha (more details of the study site in Zotz 2007b; Zotz \& Schultz 2008).

Field work in the Brazilian study sites was supported by the Federal University of Pernambuco, which has a research agreement with the landholding that owns the forest fragments. Field permit in the Panamanian study site was obtained from the Panamanian Environmental Agency (ANAM) via Smithsonian Tropical Research Institute (STRI). Field work was done in four weeks each in each country: July 2012 (Brazil) and in September/October 2012 (Panama). The surveyed period coincided with the second half of the rainy season in each forest, and thus we were able to sample branches freshly broken due to heavy storms. This was important, as fallen epiphytes may die within a few months after branchfall, but can live up to a year (Matelson et al. 1993).

\section{Branchfall}

Surveys of branches on the forest floor were conducted within randomly placed $5 \times 0.5 \mathrm{~m}$ transects (60 in Brazil, 36 in Panama). In Brazil, 30 transects were placed at least $200 \mathrm{~m}$ from the forest edge (from now on called 'Brazilian core transects'); 30 transects were placed within $60 \mathrm{~m}$ of the forest edge ('Brazilian edge transects'). In each of the 96 transects, we screened the forest floor 
for branches. We counted only branches with $>50 \%$ of their length within transects and that did not crumble due to advanced decomposition when handled. Branches were divided into four diameter classes based on the thickest internode $(0.5-2,2-4,4-6,>6 \mathrm{~cm})$. Number and size of side branches were ignored. Branches with $<0.5 \mathrm{~cm}$ diameter were surveyed in 1 x 0.5 m subplots nested and centrally located in each transect, and their number was extrapolated from the subplot to the $5 \times 0.5 \mathrm{~m}$ branch transect.

We follow the terminology presented by Moffett (2000) for the terms 'canopy' (aboveground parts, including tree crowns and trunks) and 'crown' (branches, excluding the trunk). We were not able to identify the origin of the branches found on the forest floor. Hence, although most thin branches can be assumed to have their origin in the outer crowns of overstorey trees, they could also be from understory trees and shrubs as well as from inner crowns. We did not count thin branches attached to thick ones, but it is reasonable to assume that at least some thin branches detach during descent or upon impact on the forest floor. This detachment of thin branches should thus increase their abundance on the forest floor.

\section{Epiphytes}

We extended the 96 branch transects longitudinally to $5 \times 10 \mathrm{~m}$ and surveyed vascular holoepiphytes on the forest floor. For each individual, we recorded species identity, life stage (juvenile or adult), and diameter of host branch if present. Classification as adult was based on remains of inflorescence and/or size comparable to reproductive conspecifics. We sampled all epiphyte taxa in Panama, but excluded ferns and aroids at Brazilian transects due to difficulties with species identification. The full sampling in Panama allowed the comparison between forest floor and canopy besides addressing branchfall effects on epiphyte community, whereas Brazilian transects were mainly used for addressing branchfall effects. Excluding ferns and aroids from the Panamanian transects did not change the relationships of epiphyte community (abundance, richness and proportion of adults) with branch diameter (results not shown). This suggests that the absence of ferns and aroids in the Brazilian transects should not affect the analysis of branchfall effects. A list of observed vascular holoepiphyte species is given in Table B.1.

Epiphytes species in the canopy were surveyed using a combination of ground-based observation with binoculars and tree climbing at the Brazilian sites (Table B.2). In Panama, the epiphytes occurring in the canopy had recently been surveyed in a comprehensive census from 2010-2012, in which the precise identity and the host branch diameter of every individual epiphyte was recorded (Mendieta-Leiva, Wagner \& Zotz, unpubl. data; Table B.2). 


\section{Branchfall as demographic filter for epiphyte communities}

As additional structural characteristic of each transect, we determined diameter at breast height (DBH), mean height at first branching (first ramification of the stem) and total tree height for all trees with $\mathrm{DBH}>5 \mathrm{~cm}$.

\section{Analyses}

First, we quantified mean values of key physiognomic variables of the forest per transect (number of trees, tree $\mathrm{DBH}$, height at first branching, tree height), as well as of branch abundance, epiphyte abundance and epiphyte richness on the forest floor. We additionally quantified mean values of abundance and richness for epiphytes attached to branches, detached from branches and adult individuals. For epiphytes attached to branches, we further quantified mean values of epiphyte abundance and richness per branch in each transect. We accounted for the difference in area between epiphyte and branch transects ( 50 and $2.5 \mathrm{~m}^{2}$, respectively) by multiplying the number of branches found in the branch transects by 20. For all variables, we compared the three study sites (Brazilian core, Brazilian edge, Panamanian transects) with simultaneous max- $t$ tests using Tukey contrasts that are robust under non-normality, heteroscedasticity and variable sample size (Herberich et al. 2010). For adequate comparisons, ferns and aroids were excluded from Panamanian transects.

To investigate the effect of sampling effort on species numbers, we generated species accumulation curves per study site by randomizing 100 times the increase in species richness caused by adding one transect to the sample. Species accumulation curves tending to an asymptotic value (near the actual number of species) reveal appropriate sampling effort.

With the branches on the forest floor, we addressed whether the assumption that branch abundance on the forest floor is negatively correlated with branch diameter. Due to possible nonlinear relationships with branch diameter (e.g. Hietz 1997), we used generalized additive mixedeffects models (GAMMs) with the absolute number of branches per transect as response, branch diameter class as fixed effect and transect as random effect (Zuur et al. 2009). Transect was used as random effect because branch abundances varied between transects, probably reflecting variation in age, structure and abscission patterns of local tree species. We applied negative binomial GAMMs (with log link function) to account for possible overdispersion in count data (Barry \& Welsh 2002; Zuur et al. 2009; O’Hara \& Kotze 2010). Thereafter, we addressed the hypotheses that 1) epiphyte abundance, 2) epiphyte richness and 3) proportion of adult epiphytes are positively correlated with branch diameter (Fig. 3.1). Similarly to branch abundance, we performed GAMMs with the same fixed and random effects. As response variables, we firstly assessed trends of absolute values, using absolute number of individuals per transect (referred to as absolute epiphyte abundance) and absolute number of species per transect (i.e. absolute 
epiphyte richness). In both cases, we applied negative binomial GAMMs. Secondly, to adequately test the hypotheses given potential differences in branch, we performed GAMMS controlling epiphyte abundance and richness for branch abundance per diameter class. To this end, we standardized both epiphyte abundance and richness by dividing them by branch abundance (from now on referred to as abundance per branch and richness per branch, respectively). These two variables were used as response in gamma family GAMMs with log link function (Zuur et al. 2009). Finally, the proportion of adults was used as response variable for binomial GAMMs (Zuur et al. 2009).

We further assessed whether epiphyte abundance and richness observed on the forest floor reflect the trends observed in the canopy (trunk and crowns). To address this question, we analyzed the epiphyte abundance and richness in branch diameter classes in the canopy directly above the Panamanian transects. For this purpose, we used the vascular epiphyte inventory of the crane plot (Zotz \& Schultz 2008, Mendieta-Leiva, Wagner \& Zotz, unpubl. data). From our 36 Panamanian transects, 29 had their canopy epiphytes inventoried. Similarly to the analyses of the forest floor, we applied negative binomial GAMMs (with log link function) with epiphyte abundance and richness as response variables, branch diameter class as fixed effect and transect as random effect (Zuur et al. 2009). Additionally, we used Spearman correlations to test whether abundance and richness on the forest floor were correlated with their canopy counterparts. For these correlations, two analyses were performed: i) per transect (all epiphytes found on the forest floor and inventoried in the canopy) and ii) per transect and per branch diameter class (only epiphytes found on the forest floor attached to branches and canopy epiphytes on substrate with the same thickness distribution as in the forest floor). Thereafter, we assessed the proportion of the epiphytes over branch diameter found on the forest floor in relation to the entire transect (floor and canopy). For this analysis, we applied binomial GAMMs with the proportion of individuals and species on the forest floor as response variables, branch diameter class as fixed effect and transect as random effect (Zuur et al. 2009).

The species composition found on the forest floor was compared with that from the inventoried canopy above Panamanian transects. We compared 1) all epiphytes and 2) epiphytes found only on branches $<10 \mathrm{~cm}$ in diameter. Initially, we built a species per transect matrix with abundances separately for ground and for canopy individuals. To avoid bias due to low richness in the forest floor per transect but still retain a reasonable number of transects, we included only transects with at least two species on the ground $(\mathrm{n}=18$ considering all epiphytes, $\mathrm{n}=17$ considering only epiphytes on branches $<10 \mathrm{~cm}$ in diameter). We performed a non-metric multidimensional scaling (NMDS) and plotted the resulting ordination showing separate convex hulls for ground and canopy. For this analysis, we estimated a dissimilarity matrix (Bray-Curtis index) between transects. Thereafter, we used this dissimilarity matrix to perform an analysis of similarities 


\section{Branchfall as demographic filter for epiphyte communities}

(ANOSIM) between ground and canopy. We then assessed which species were responsible for significant differences between ground and canopy by performing a Dufrene-Legendre indicator species analysis (Dufrêne \& Legendre 1997). Finally, we tested whether paired ground and canopy transects were more similar than expected by chance. For this analysis, we estimated the Bray-Curtis dissimilarity index for each transect pair and for random pairs $(n=18$ pairs considering all epiphytes, $\mathrm{n}=17$ considering only epiphytes on branches $<10 \mathrm{~cm}$ in diameter). The dissimilarity of each random pair was an average of the dissimilarity between each ground sample and $\mathrm{n}$ random canopy samples other than its actual canopy sample. We then compared the mean dissimilarity between actual vs. random pairs with simultaneous max- $t$ tests using Tukey contrasts (Herberich et al. 2010).

All analyses were done in $\mathrm{R}$ (version 3.0.1). GAMMs were implemented using the R library 'mgcv' version 1.7-24 (Wood 2011). Ordination, dissimilarity matrices and analysis of similarities were implemented using the R library 'vegan', whereas Dufrene-Legendre indicator species analysis used the library 'labdsv'.

\subsection{Results}

In total, we counted $>24,000$ branches at the two sites. Brazilian core transects had $325 \pm 284$ (mean $\pm \mathrm{SD}, \mathrm{n}=30)$ branches per transect, Brazilian edge transects had $224( \pm 102, \mathrm{n}=30)$ branches per transect and the 36 Panamanian transects $220( \pm 169)$ branches per transect (equivalent to an average of 130, 90 and 88 branches per $\mathrm{m}^{2}$, respectively). Although Brazilian core transects had, on average, the highest number of branches, branch abundance did not differ significantly between study sites (Table 3.1; see Table B.3 for an extended version of Table 3.1). At all three study sites, the abundance of the thinnest branches was significantly higher than those of thicker ones, with $>90 \%$ of all branches belonging to the first two diameter classes $(<0.5 \mathrm{~cm}$ and 0.5-2 cm, Fig. 3.2; Table 3.1). 


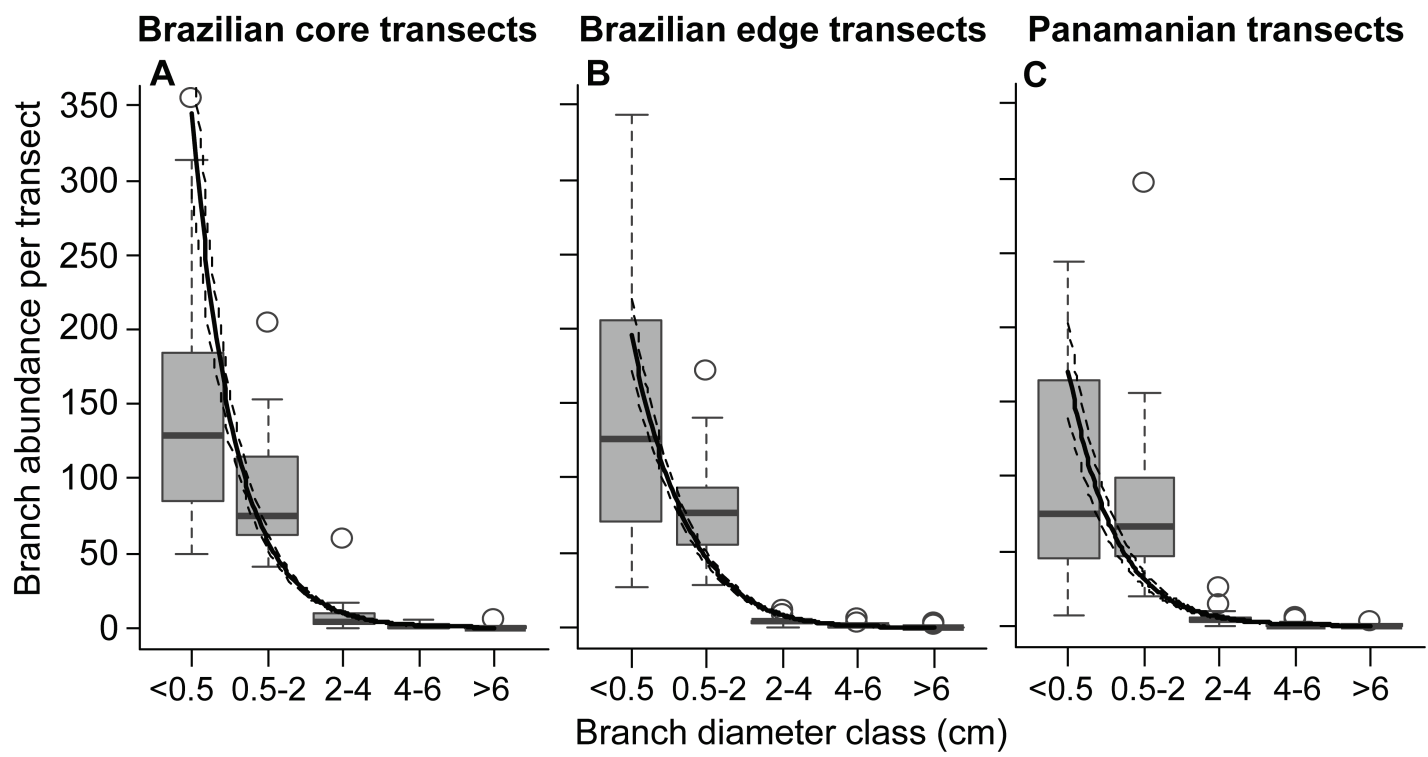

Figure 3.2. Branch abundances as a function of branch diameter. A) Brazilian core transects $(n=30)$. B) Brazilian edge transects $(n=30)$. C) Panamanian transects $(n=36)$. Box-plots show the median as central line, the first and third quantiles as the bottom and top box limits, 1.5 interquantile range as whiskers, and outliers as circles. Solid lines show fits from GAMMs with 95\% CI indicated by dashed lines. 
Table 3.1. Comparisons between study sites. Several measures characterizing forest structure, branch abundance, epiphyte abundance and epiphyte richness. Total numbers and means \pm SD per study site are provided. Percentages of adults and epiphytes attached to branches to the study site totals are given in parentheses. Means were compared with simultaneous max-t tests using Tukey contrasts that are robust under non-normality, heteroscedasticity and variable sample size. Significantly different means are indicated by different letters representing pairwise differences. Note that for epiphytes, only mean values for Panamanian transects without ferns and aroids were used in the comparisons with Brazilian study sites. The symbol $\uparrow$ indicates $n=35$ (excluding one transect without trees).

\begin{tabular}{|c|c|c|c|c|c|}
\hline \multirow[t]{2}{*}{ Variable } & & \multirow{2}{*}{$\begin{array}{r}\text { Brazilian core } \\
\text { transects }(n=30)\end{array}$} & \multirow{2}{*}{$\begin{array}{r}\text { Brazilian edge } \\
\text { transects }(n=30)\end{array}$} & \multicolumn{2}{|c|}{ Panamanian transects $(n=36)$} \\
\hline & & & & aroids & All species \\
\hline \multirow[t]{7}{*}{ Trees } & Mean number per transect & $5.1 \pm 2.1 \mathrm{ab}$ & $6.4 \pm 3.2 \mathrm{a}$ & $4.8 \pm 2.1 \mathrm{~b}$ & \\
\hline & Mean DBH (m) & $0.2 \pm 0.1 \mathrm{a}$ & $0.18 \pm 0.06 \mathrm{~b}$ & $0.16 \pm 0.06$ & \\
\hline & & & & $\mathrm{b} \dagger$ & \\
\hline & Mean height & $8.2 \pm 2.0 \mathrm{a}$ & $4.7 \pm 2.0 \mathrm{~b}$ & $8.0 \pm 2.0$ & \\
\hline & first branching (m) & & & $\mathrm{a}^{\dagger}$ & \\
\hline & Mean height (m) & $15.4 \pm 3.4 \mathrm{a}$ & $10.9 \pm 2.7 \mathrm{~b}$ & $13.2 \pm 2.6$ & \\
\hline & & & & $\mathrm{c} \dagger$ & \\
\hline Branch & Total & 9759 & 6721 & 7939 & \\
\hline \multirow[t]{6}{*}{ abundance } & Mean per transect & $325 \pm 284 \mathrm{a}$ & $224 \pm 102 \mathrm{a}$ & $220 \pm 169$ & \\
\hline & & & & $\mathrm{a}$ & \\
\hline & Mean per transect $(<0.5 \mathrm{~cm}$ & $215 \pm 237 \mathrm{a}$ & $142 \pm 85 \mathrm{a}$ & $135 \pm 151$ & \\
\hline & diameter) & & & $\mathrm{a}$ & \\
\hline & Mean per transect $(<2 \mathrm{~cm}$ & $316 \pm 283 \mathrm{a}$ & $219 \pm 101 \mathrm{a}$ & $214 \pm 168$ & \\
\hline & diameter) & & & a & \\
\hline Epiphyte & Total & 546 & 349 & 164 & 232 \\
\hline \multirow[t]{5}{*}{ abundance } & Total adults & $211(39 \%)$ & $153(44 \%)$ & $86(52 \%)$ & $101(44 \%)$ \\
\hline & Total attached to branches & $367(67 \%)$ & $260(74 \%)$ & $112(68 \%)$ & $164(71 \%)$ \\
\hline & Mean per transect & $18.2 \pm 20.5 \mathrm{a}$ & $11.6 \pm 17.8 \mathrm{ab}$ & $4.6 \pm 7.1 \mathrm{~b}$ & $6.4 \pm 9.8$ \\
\hline & $\begin{array}{l}\text { Mean attached to branches } \\
\text { per transect }\end{array}$ & $12.2 \pm 17.0 \mathrm{a}$ & $8.7 \pm 16.8 \mathrm{ab}$ & $3.1 \pm 5.1 \mathrm{~b}$ & $4.6 \pm 6.4$ \\
\hline & $\begin{array}{l}\text { Mean attached to branches } \\
\text { per transect per branch }\end{array}$ & $\begin{array}{r}2.810^{-3} \pm 3.510^{-3} \\
\mathrm{a}\end{array}$ & $2.310^{-3} \pm 4.510^{-3} \mathrm{a}$ & $\begin{array}{r}1.110^{-3} \pm 2.0 \\
10^{-3} \mathrm{a}\end{array}$ & $1.810^{-3} \pm 3.110^{-3}$ \\
\hline Epiphyte & Total & 23 & 16 & 27 & 39 \\
\hline \multirow[t]{5}{*}{ richness } & Total adults & $21(91 \%)$ & $14(88 \%)$ & $17(63 \%)$ & $24(62 \%)$ \\
\hline & Total attached to branches & $17(74 \%)$ & $13(81 \%)$ & $18(67 \%)$ & $29(74 \%)$ \\
\hline & Mean per transect & $5.1 \pm 3.2 \mathrm{a}$ & $2.3 \pm 1.9 \mathrm{~b}$ & $1.9 \pm 2.3 \mathrm{~b}$ & $2.8 \pm 3.6$ \\
\hline & $\begin{array}{l}\text { Mean attached to branches } \\
\text { per transect }\end{array}$ & $3.3 \pm 2.5 \mathrm{a}$ & $1.8 \pm 1.8 \mathrm{~b}$ & $1.4 \pm 1.6 \mathrm{~b}$ & $2.2 \pm 2.3$ \\
\hline & $\begin{array}{l}\text { Mean attached to branches } \\
\text { per transect per branch }\end{array}$ & $\begin{array}{r}7.710^{-4} \pm 7.110^{-4} \\
a\end{array}$ & $5.210^{-4} \pm 5.510^{-4} \mathrm{a}$ & $\begin{array}{r}5.310^{-4} \pm 7.5 \\
10^{-4} \mathrm{a}\end{array}$ & $7.310^{-4} \pm 7.910^{-4}$ \\
\hline
\end{tabular}

We found a total of 546 individuals of 23 epiphyte species in Brazilian core, 349 individuals of 16 species in Brazilian edge, and 232 individuals of 39 species in Panamanian transects (Table 1; see Table B.1 for species lists). Overall, the transects captured a considerable proportion of the epiphyte species found in the forests of the respective study sites (36-52\%, Fig. 3.3). When 
considering only the species in transects' canopies, a larger proportion of epiphytes species was found on the forest floor (49-89\%, Fig. 3.3). Excluding ferns and aroids of the Panamanian transects for comparisons between study sites, absolute epiphyte abundance was significantly higher in Brazilian core transects (ca. 18 individuals per transect) compared to Brazilian edge (12 individuals per transect) and Panamanian (5 individuals per transect) transects (Table 3.1; equivalent to ca. $0.36,0.23$ and 0.11 individuals per $\mathrm{m}^{2}$, respectively). Similarly, the average absolute species richness per transect was significantly higher in Brazilian core transects (ca. 5 species per transect) than in Brazilian edge ( 2.3 species per transect) and Panamanian ( 2 species per transect) transects (Table 3.1; equivalent to ca. $0.1,0.05$ and 0.04 species per $\mathrm{m}^{2}$, respectively). However, differences in epiphyte abundance and richness per branch were not significant (Table $3.1)$.
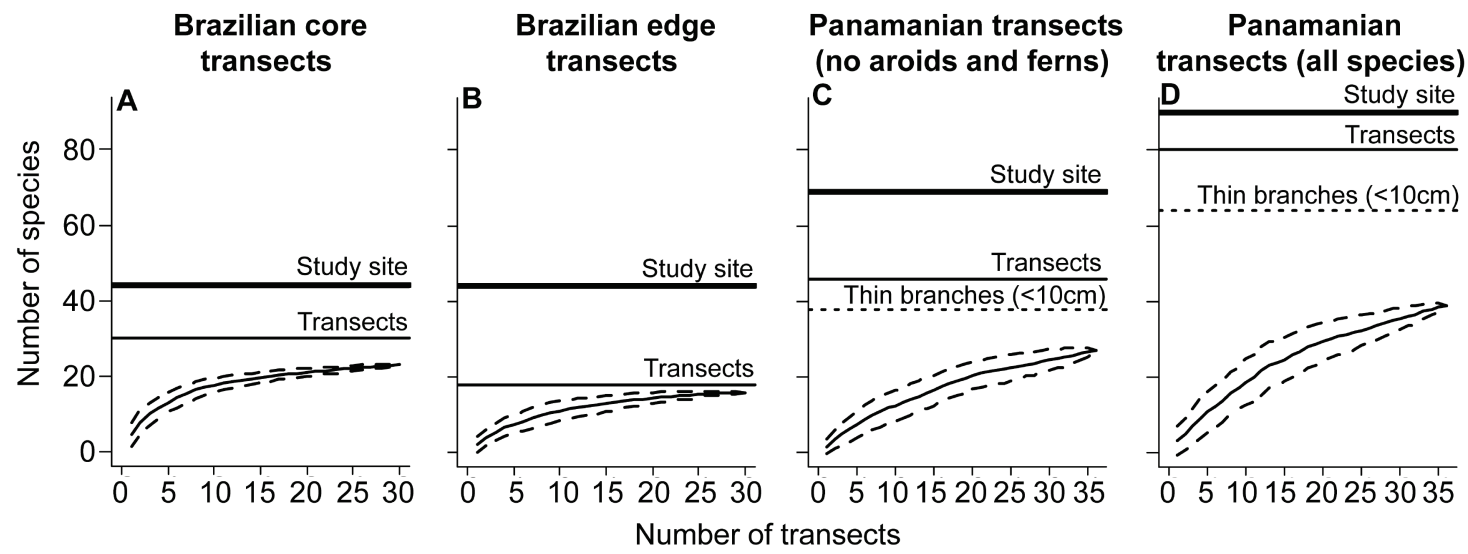

Figure 3.3. Species accumulation curves based on forest floor-based sampling of epiphytes. A) Brazilian core transects $(n=30)$. B) Brazilian edge transects $(n=30)$. C) Panamanian transects, excluding ferns and aroids $(n=36)$. D) Panamanian transects, all species $(n=36)$. Solid curves give the mean number of species based on 100 randomized samplings, dashed curves the estimated $95 \%$ CI. Horizontal lines indicate the number of species present in the canopy of the transects (thin lines), in the study site (thick lines, same estimate for both Brazilian study sites) and on substrate $<10 \mathrm{~cm}$ in diameter (dot line, in c and d). See Table B.1 for the list of species found in the transects and Table B.2 for species lists found in the study sites. Note that the Brazilian study sites showed curves leveling off, whereas the Panamanian site revealed a slightly steeper curve in agreement with the higher number of species.

Most individuals fell attached to branches ( $>65 \%)$, particularly in the Brazilian edge transects (Table 3.1). Epiphytes detached from branches were often attached to bark pieces, canopy soil or moss mats. Between $39 \%$ and $52 \%$ of the individuals on the forest floor were adults Table 3.1). Adults were found for most of the species, particularly at the Brazilian core transects $(91 \%$ of species; Table 3.1). Remarkably, the proportion of adults among those individuals detached from branches was much higher (56\% in Brazilian core, $64 \%$ in Brazilian edge, and $72 \%$ in Panamanian transects) than among those attached to branches (30\% in Brazilian core, $37 \%$ in Brazilian edge, and 36\% in Panamanian transects; see Table B.3 for total numbers). 
The relationship between absolute epiphyte abundance or richness and branch diameter differed in the three study sites (Fig. 3.4; see Table B.4 for summary statistics). Absolute epiphyte abundance showed a hump-shaped relationship with increasing branch diameter in Brazilian core transects (Fig. 3.4a), with no clear relationship in Brazilian edge transects (Fig. 3.4b) and a positive relationship in Panamanian transects (Fig. 3.4c). Absolute species richness showed a hump-shaped relationship with increasing branch diameter at both Brazilian study sites (Fig. 3.4de) and a positive relationship in the Panamanian transects (Fig. 3.4f). In contrast to these trends, the abundance (Fig. 3.5a-c) and richness (Fig. 3.5d-f) per branch showed a positive relationship with branch diameter at all three study sites (Fig. 3.5, Table B.4). There was a positive relationship between proportion of adults and branch diameter at all three study sites (Fig. 3.6, Table B.4).

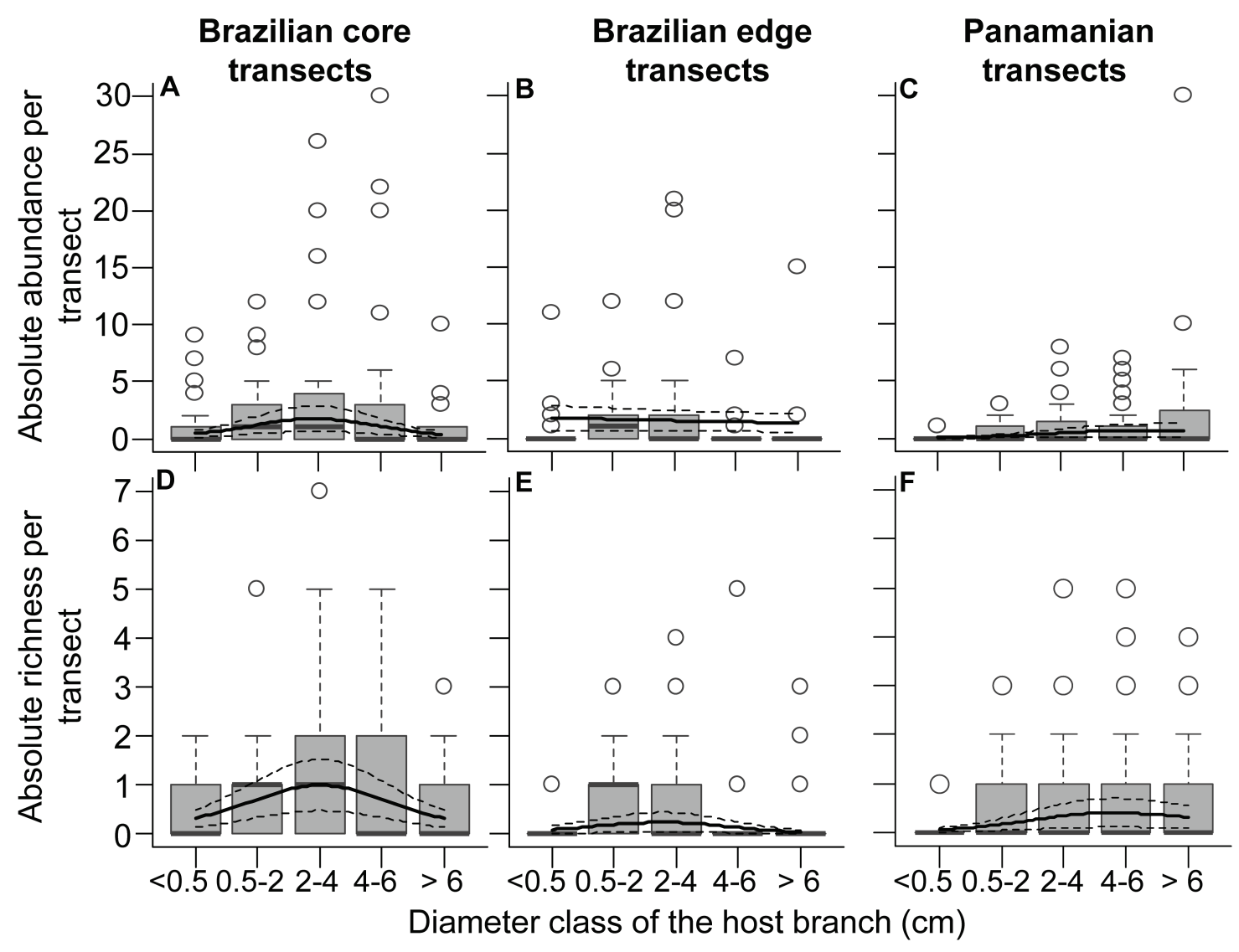

Figure 3.4. Absolute epiphyte abundance (A-C) and richness (D-F) per transect as a function of branch diameter. Trends are shown for Brazilian core (A,D, $n=30)$, Brazilian edge (B,E, $n=30)$, and Panamanian $(C, F, n=36)$ transects. Box-plots show the median as central line, 1.5 interquantile range as whiskers, and outliers as circles. Solid lines give the values predicted by the estimated GAMMs, dashed lines show $95 \%$ CI. Note that the number of epiphytes was generally hump-shaped along diameter classes in Brazilian transects, whereas it was positive in Panamanian transects. 


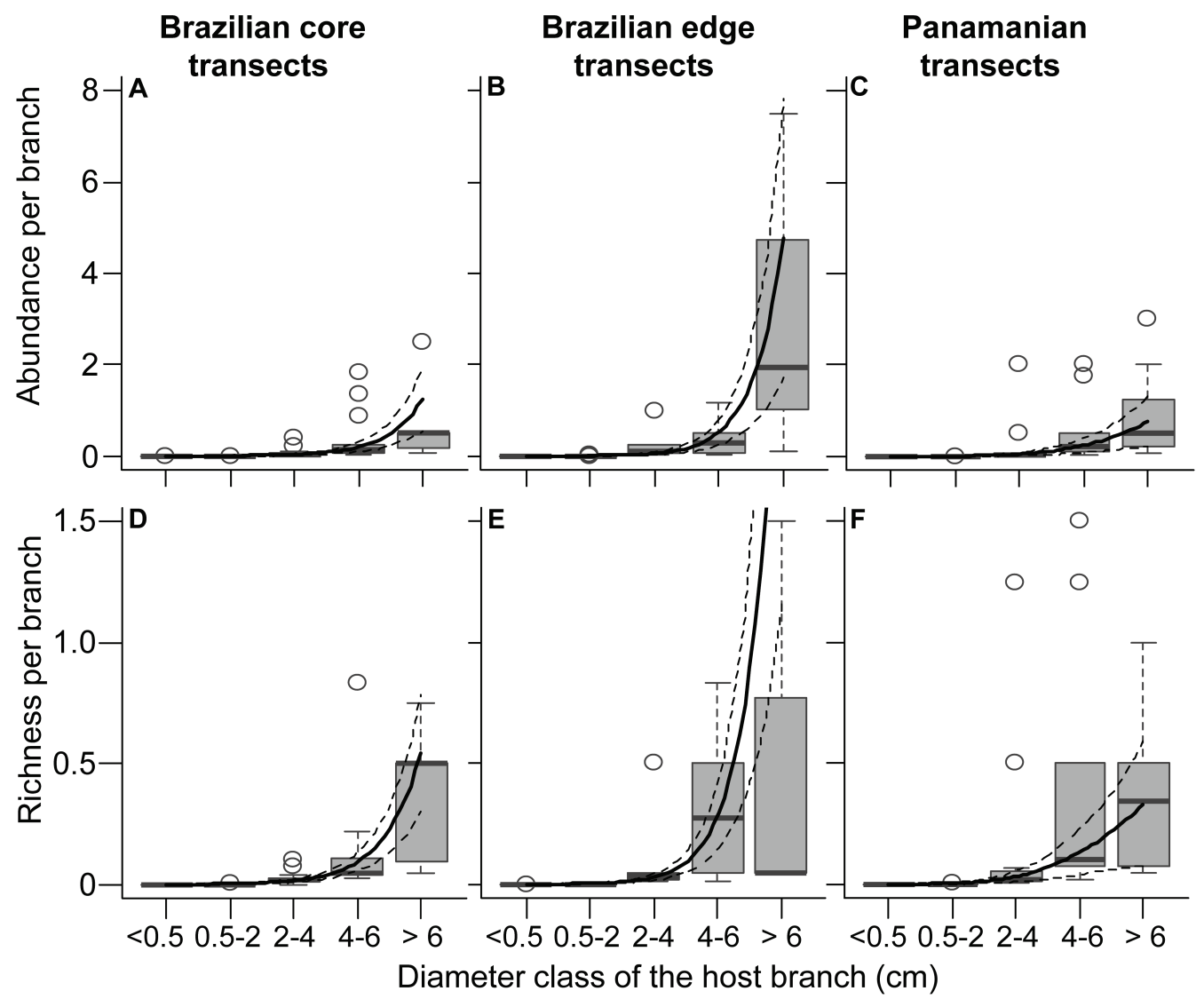

Figure 3.5. Epiphyte abundance (A-C) and richness (D-F) per branch as a function of branch diameter. Trends are shown for Brazilian core (A,D, n=26), Brazilian edge (B,E, n=21), and Panamanian $(C, F, n=25)$ transects. Box-plots show the median as central line, 1.5 interquantile range as whiskers, and outliers as circles. Solid lines give the values predicted by the estimated GAMMs. Dashed lines show the estimated 95\% CI.

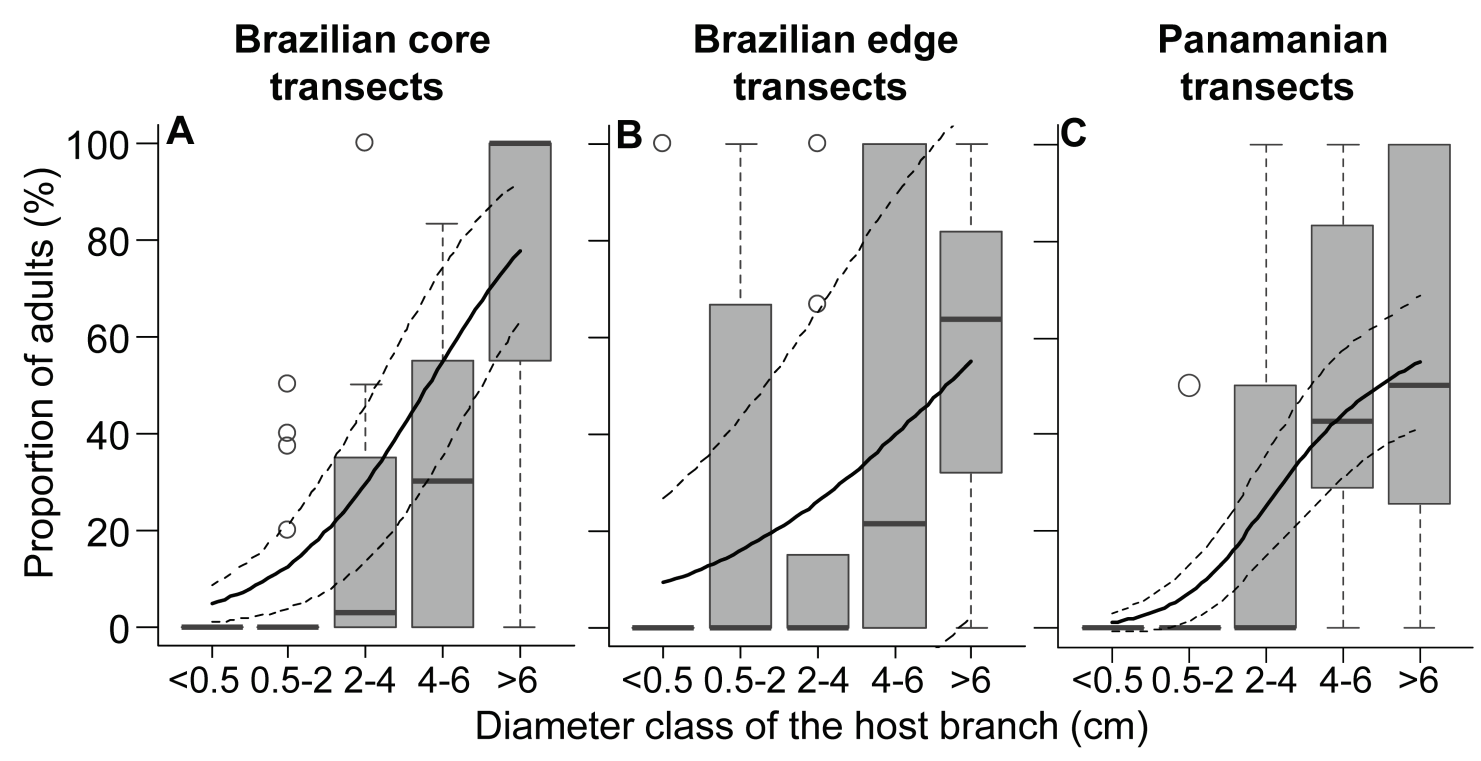

Figure 3.6. Proportion of adults as a function of branch diameter. A) Brazilian core transects $(n=30)$. B) Brazilian edge transects $(n=30)$. C) Panamanian transects $(n=36)$. Box-plots show the median as central line, 1.5 interquantile range as whiskers, and outliers as circles. Solid lines give the values predicted by the estimated GAMMs. Dashed lines show the estimated $95 \%$ CI. 


\section{Branchfall as demographic filter for epiphyte communities}

The inventoried canopy above our Panamanian transects had 4386 epiphyte individuals (corresponding to 151 individuals per transect or $\sim 3$ individuals per $\mathrm{m}^{2}$ ) of 80 species. Considering only substrates with diameters comparable to those found on the forest floor $(<10$ $\mathrm{cm}$ ), the inventoried canopies above our transects hosted 866 epiphyte individuals (30 individuals per transect, 0.6 individuals per $\mathrm{m}^{2}$ ) belonging to 64 species ( $20 \%$ of all individuals and $80 \%$ of all species $)$. Epiphyte abundance $\left(P<0.001, \mathrm{df}_{\mathrm{eff}}=1.98\right)$ and richness $\left(P<0.001, \mathrm{df}_{\mathrm{eff}}=1.97\right)$ above the Panamanian transects were positively related to increasing branch diameter (Fig. 3.7ab). There was no correlation between the number of individuals and species on the forest floor and in the canopy. Across Panamanian transects, epiphytes on the forest floor (either attached to or detached from branches) corresponded to c. $4 \%$ of total number of individuals and to $48 \%$ of the species found. Considering only individuals found attached to branches ( $<10 \mathrm{~cm}$ in diameter), this proportion was of $13 \%$ for individuals and $40 \%$ for species, gradually decreasing with branch diameter for individuals $\left(P<0.05, \mathrm{df}_{\mathrm{eff}}=1.53\right)$ and species $\left(P<0.001, \mathrm{df}_{\mathrm{eff}}=1.00\right.$; Fig. $\left.3.7 \mathrm{c}-\mathrm{d}\right)$. Species composition differed significantly between ground and canopy for all epiphytes $(P=$ 0.001 , ANOSIM statistic $\mathrm{R}=0.37)$ and for epiphytes on substrate $<10 \mathrm{~cm}$ in diameter $(P=0.001$, ANOSIM statistic $R=0.29$ ). Species composition on the ground was more variable than in the canopy (Fig. 3.7e-f), particularly considering all epiphytes (Fig. 3.7e). Most indicator species of these compositional differences were aroids and ferns found only or mostly in the canopy (Table B.5). Mean species similarity between actual ground and canopy transect pairs was not significantly different from the mean species similarity between random ground and canopy pairs. 

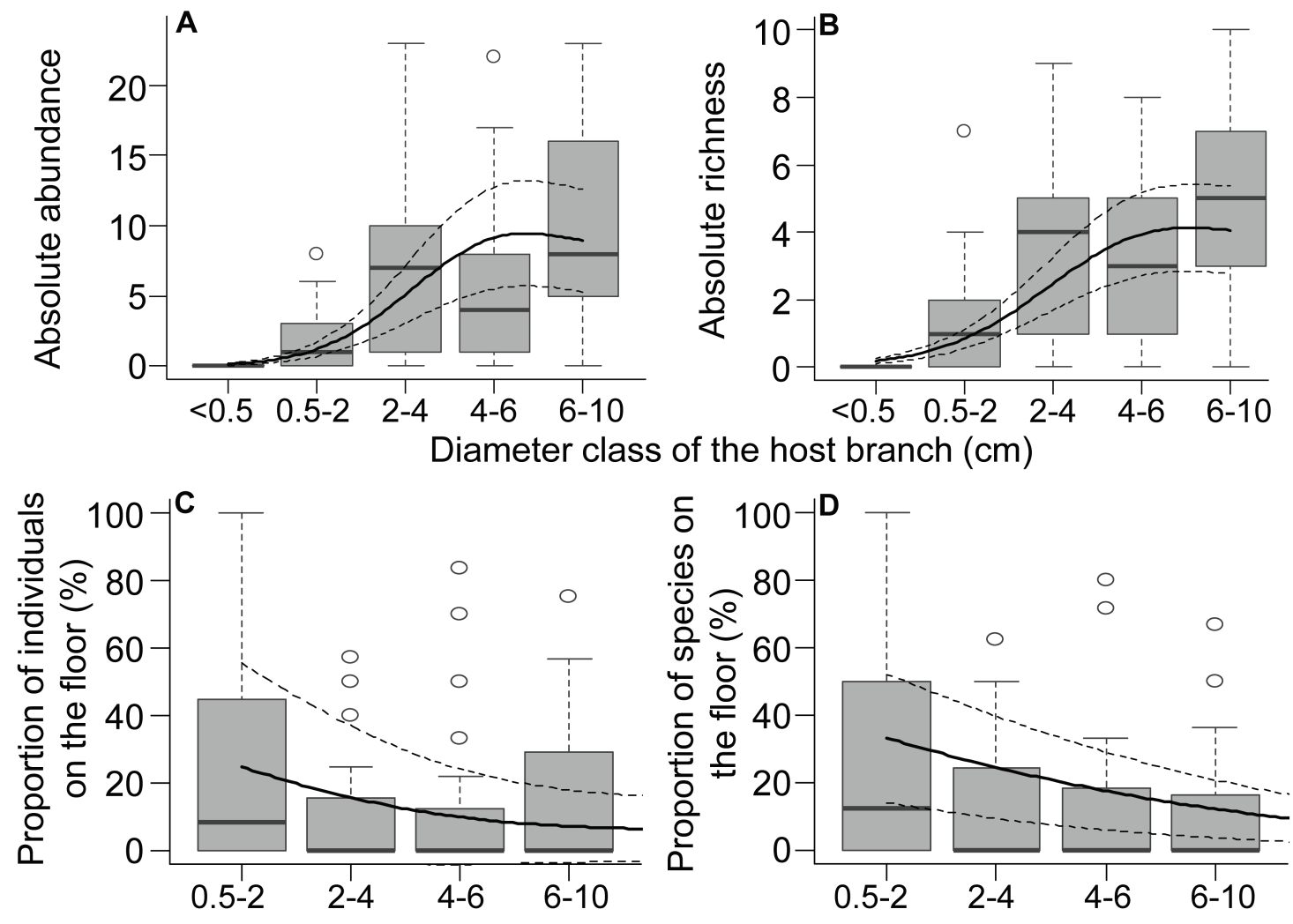

Diameter class of the host branch $(\mathrm{cm})$
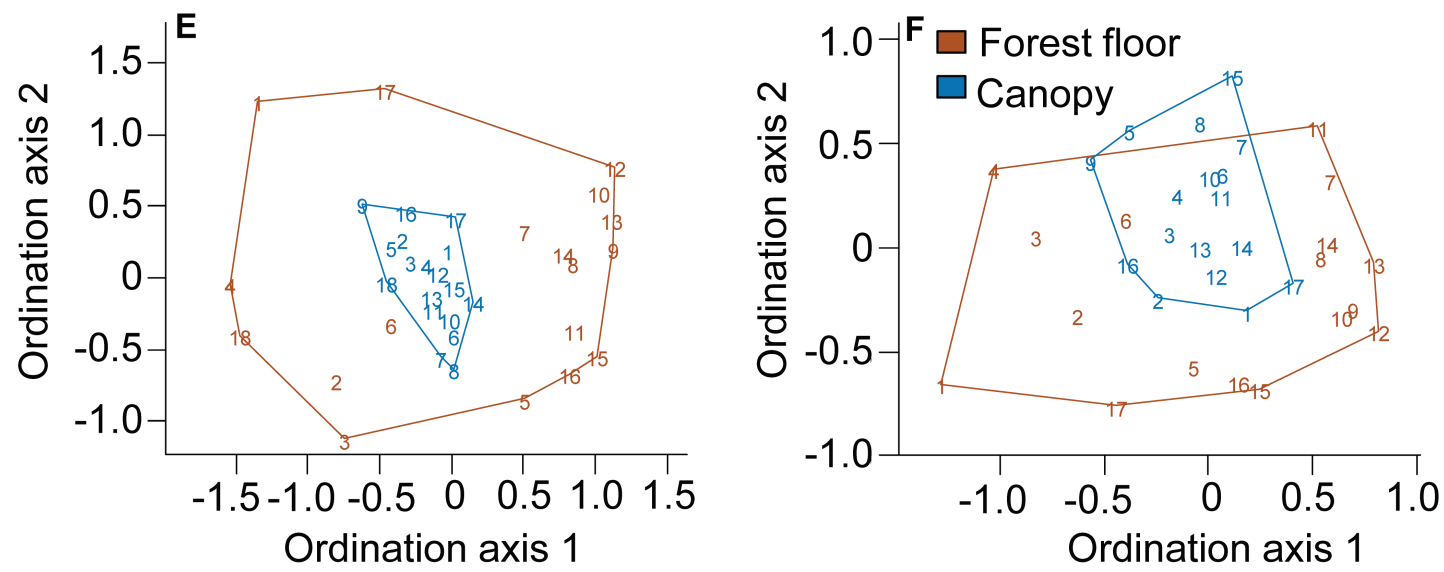

Figure 3.7. Epiphytes in the canopy and their relationship with forest floor trends. (A) Epiphyte abundance and (B) species richness in the canopy directly above the Panamanian transects $(n=29)$ as a function of branch diameter. Proportion of individuals (C) and species (D) found on the forest floor compared to the transects' total abundance (forest floor and canopy). (E-F) Non-metric multidimensional scaling of transects based on species composition and abundance considering (E) all individuals found on the forest floor and canopy ( $\mathrm{n}=18$ forest floor and canopy pairs) and $(\mathrm{F})$ only individuals on substrate $<10 \mathrm{~cm}$ in diameter $(\mathrm{n}=17)$. Forest floor and canopy pairs are indicated by the same numbers in E-F (legend in F). See S2 File for the number of individuals and species censed within the whole crane plot (ca. 0.9 ha). Solid lines give the values predicted by the estimated GAMMs, whereas dashed lines show the estimated 95\% CI in A-D. Lines connecting numbers indicate convex hulls in D-F. We excluded the thinnest branch diameter class in $\mathrm{C}$ and $\mathrm{D}$ due to overall low abundances in the canopy (see A-B). Box-plots show the median as central line, 1.5 interquantile range as whiskers, and outliers as circles. 


\subsection{Discussion}

\section{Surveying epiphytes on the forest floor}

A considerable proportion of the species above the transects was also found on the forest floor (Fig. 3.3 and Fig. 3.7c-d). Single transects entailed random subsets of the epiphyte community in the canopy (Fig. 3.7e-f). Although this may be the main limitation of this method, at least some species typically restricted to stable substrates (i.e. tree trunks and inner crowns, Zotz 2007b; Woods et al. 2015) were found on the forest floor. In fact, most of the individuals found detached from branches were attached to substrate parts (e.g. bark pieces or canopy soil) and thus may have fallen from trunks and inner crowns. Most indicator species for the Panamanian canopy transects preferentially occur, however, on trunks and inner-crowns (e.g. Trichomanes spp., Campyloneurum spp., Anthurium spp., Dichaea panamensis; full list in Table B.5). Hence, to increase the effectiveness and completeness, forest floor-based surveys could target transects with fallen trees or near old trees, as old trees have been indicated to host a higher number of species and should always be included in epiphyte surveys (Shaw \& Bergstrom 1997; Zotz \& Bader 2011).

Sampling the forest floor might be particularly useful for investigating epiphytes occurring on branches $<10 \mathrm{~cm}$ in diameter. This is illustrated by the fact that there were considerably fewer canopy indicator species when limiting the comparison between canopy and forest floor to branches $<10 \mathrm{~cm}$ in diameter than when considering the entire canopy (Table B.5). This is also supported by the lower species number (Fig. 3.3c-d) and variation in composition (Fig. 3.7e-f) compared to similar analyses considering epiphytes of the entire canopy. Furthermore, an unexpected high proportion of epiphytes on branches $<10 \mathrm{~cm}$ in diameter were on the forest floor ( $>12 \%$ all individuals belonging to $40 \%$ of all species, see also Fig. 3.7c-d for averages over each diameter class). This is important because these thin branches, often located in the outer crowns, are the most difficult canopy habitats to access despite hosting a sizable portion of individuals and species ( $20 \%$ and $80 \%$, respectively, for Panamanian transects).

Besides floristic information, the forest floor proved to be an important source of information on epiphyte demography and community structure. The fact that the patterns of epiphyte abundance and richness over branch diameter found on the forest floor mirrored the community structure of the canopy (compare Figs. 3.4, 3.5 and 3.7) indicates the community structure on the canopy can be surveyed in the forest floor. Hence, demographical inferences can be attempted with a survey method that is faster, cheaper and safer than commonly applied techniques, such as tree climbing and research cranes (see also Mondragón \& Ticktin 2011). This is valuable information, considering that even if floristic data from the forest floor might not be as complete as from tree climbing, the forest floor offers much needed demographic data. Furthermore, besides surveys 
focusing on economic value of fallen epiphytes (Mondragón \& Ticktin 2011; Toledo-Aceves et al. 2014), further studies incorporating forest floor information can focus on combining demography and community structure with substrate characteristics (see next section).

\section{Epiphyte fall and branch diameter}

We found a high density of epiphytes on the forest floor (1100-3600 individuals per hectare). The fact that most epiphytes on the forest floor were found attached to branches emphasizes the importance of branchfall as a cause of epiphyte mortality. Although we have not directly measured mortality rates via falling with or from branches (Nadkarni \& Matelson 1992), indirect estimates are possible if epiphytes in the canopy have been inventoried, as in our Panamanian study site. In this case, the mortality rate caused by falling with or from branches would be at least $4 \%$ per year (percentage of individuals found on the forest floor), considering that the majority of epiphytes on the forest floor dies within less than one year (Matelson et al. 1993). Our estimate is lower the annual mortality rate reported for a humid montane forest via monitoring selected branches with photographs (16\%, Hietz 1997). However, epiphyte abundances in the canopy of the Panamanian study site were generally low compared to montane cloud forests (Nadkarni \& Matelson 1992; Freiberg \& Freiberg 2000), which may contribute to branchfall (Zotz et al. 2005).

When considering only epiphytes falling with branches, absolute epiphyte abundance and richness revealed site-specific types of relationships with branch diameter (Fig. 3.4). As such differences disappeared after accounting for branch abundance (Fig. 3.5), they likely reflect local differences in branch dynamics. The resulting epiphyte abundance and richness per branch supported the hypotheses of higher abundance and richness on thick branches (compare Figs. 3.1 and 3.5). The main explanation for higher epiphyte abundance and richness on branches of larger diameter classes is lower epiphyte mortality via branchfall (also found by Hietz 1997) and more time for colonization. In fact, branchfall was identified as main cause of epiphyte fall (Table 3.1) and the assumption that thin branches are more abundant on the forest floor than thicker ones was confirmed (Fig. 3.2; Hallé, Oldeman \& Tomlinson 1978; Addicott 1991; Rust \& Roloff 2004; Zotz et al. 2005). Furthermore, thicker branches support a micro-environment that is more suitable for the epiphytic lifestyle, with lower mortality at the seedling stage due lower exposure to wind, high radiation and water stress (Wagner et al. 2013) and more suitable substrate properties, such as higher moss cover, humus volume and humus layer thickness (Freiberg 1996; Woods et al. 2015). As a consequence, epiphyte richness, cover and biomass are usually higher on the thicker branches of the inner crowns (Freiberg 1996; Hietz 1997; Zotz 2007). Accordingly, higher epiphyte abundance and richness on thicker rather than thin branches were also observed 


\section{Branchfall as demographic filter for epiphyte communities}

in the canopy at the Panamanian site (compare Figs. 3.4, 3.5 and 3.7a-b). However, quantifying branch abundance, as done for the forest floor but not for the canopy, seems essential to account for the effects of site-specific branch dynamics on the gradients of epiphyte abundance and richness over branch (compare Figs. 3.4 and 3.5).

Our hypothesis of a positive correlation between the proportion of adults and branch diameter was also supported (compare Figs. 3.1 and 3.6), indicating a strong role of branchfall on the spatial structuring of epiphyte populations. Consequently, most adults in the outer crowns are twig epiphytes with fast life-cycles (Chase 1986, 1987). In fact, most adults in the two thinnest diameter classes were from small species classifying as twig epiphytes: Campylocentrum crassyrhyzum, Rodriguezia bahiensis and Notylia lyrata in Brazilian transects as well as Campylocentrum micranthum in Panamanian transects.

\section{Study sites}

We found small site-related differences in total species richness (Table 3.1) and in speciesaccumulation curves (Fig. 3.3). The lower total species richness of edge transects was associated with the fact that most species absent in the edge have long life cycles, requiring at least 10 or more years to reproduce (e.g. Maxillaria ochroleuca, Prosthechea fragrans - first author's observations based on pseudobulb and inflorescence skeletons), or are probably less tolerant to water-stress (e.g. Anathallis sclerophylla, Acianthera pernambucensis, which were observed only on moss-rich shaded substrate). Consequently, the lack of large, stable, old trees and dominance of fast-growing pioneer trees at the same studied edge compared to core site (Oliveira-Filho, de Mello \& Scolforo 1997; Tabarelli, Mantovani \& Peres 1999) may reduce the establishment and survival of late-maturing and moisture-demanding epiphytes due to greater substrate dynamics and drier microclimate (Einzmann et al. 2015). Such lower colonization would explain why our forest floor-based sampling detected almost all species present in the canopy of edge transects but not in that of core transects (Fig. 3.3). However, because we only have one edge and core pair, we cannot statistically compare edge vs. core due to pseudoreplication, and thus further studies incorporating more pairs are necessary to investigate to what extent edge conditions affect epiphyte community composition.

The total observed epiphyte richness at the Panamanian study site in turn was slightly higher than at the Brazilian core (Table 3.1, Fig. 3.3). However, at the transect scale, Panamanian transects had fewer species than the Brazilian transects (Table 3.1). High total species richness but low richness at the transect scale indicates a high spatial turnover of epiphyte species in Panama. This high turnover might be associated with increasing turnover of aboveground biomass with decreasing elevation (Raich et al. 2006) . Considering single trees or branches as habitat patches, 
local communities are colonized by species occurring in adjacent patches (higher recruitment near source areas - Leibold et al. 2004). Hence, an increase in turnover of such patches might preclude the accumulation of species. This idea is supported by the high number of fallen trees and gaps observed in and outside the crane plot, which suggests a high rate of patch turnover, effectively limiting species accumulation. Similarly to the edge vs. core comparison, the interpretation of the differences between Brazilian core and Panamanian transects is limited due to low number of study sites. Further studies including forests along environmental and productivity gradients are necessary for a better assessment of the relationship between aboveground biomass turnover of trees and epiphyte communities. Alternatively, studies could assess such relationship by incorporating age as an additional substrate characteristic, as substrates with similar diameter may differ in age and thus time available for colonization. While data on age of tropical trees are scarce, this topic has received increasing attention, with age estimation methods spanning from allometric relationships, over counting rings to isotope dating (Lieberman et al. 1985; Fichtler, Clark \& Worbes 2003; Metcalf et al. 2009). While this much useful data is still not largely available, studies monitoring epiphytes information could extend their scope to monitor branches (with and without epiphytes). This branch monitoring would provide data on the time of occurrence of key events of substrate dynamics, such as formation, diameter growth and fall of branches. Hence, monitoring branches since their formation, and thus knowing their age, would give the time that these branches had been available for epiphyte colonization. If branches are also monitored on the forest floor, a complete appraisal of branch dynamics could provide further insights into the role of branch dynamics to epiphyte communities.

\section{Conclusion}

Sampling the forest floor for epiphytes constitutes a fast method that can provide, besides floristic data, useful information on epiphyte diversity, community composition and structure, as highlighted by the comparisons with canopy data as shown in our Panamanian transects. Furthermore, by requiring less work and training efforts as well as being cheaper and safer than climbing techniques and canopy cranes, this method can open new avenues for investigations of epiphyte demography. This is particularly valuable for the epiphyte community occurring in the least accessible, thinnest branches of the canopy. In this sense, our results confirmed branchfall as a main cause of epiphyte fall. This effect poses demographic constraints on epiphyte populations by increasing mortality (see also Hietz 1997) and by reducing time for colonization and for sexual maturation. Consequently, branchfall acts as a strong demographical filter for epiphyte populations. Moreover, branch diameter is a key factor of this demographic filter because branchfall decreases with branch diameter. This is truly independent of local forest dynamics, making demographic filtering greatest in the thin branches of the canopy. In this highly 


\section{Branchfall as demographic filter for epiphyte communities}

dynamic environment, only small, stress-tolerant and fast growing species are able to recruit, survive and reproduce. Therefore, branchfall seems to play a key role in structuring the spatial distribution of epiphytic communities.

\section{Acknowledgements}

The authors thank Yael Kisel, Ben Bond-Lamberty and two anonymous reviewers for comments on the manuscript, the Smithsonian Tropical Research Institution for the use of facilities in Panama and the Centro de Pesquisa Ambientais do Nordeste (CEPAN) as well as Prof. Marcelo Tabarelli (Federal University of Pernambuco, Recife, Brazil) for the use of facilities in Brazil. 
4 Modelling the long-term dynamics of tropical forests using functional-structural tree models: Leaf trait trade-offs as predictor of whole-tree life history variations

Gunnar Petter, Yongzhi Ong, Gerhard Zotz, Holger Kreft, Juliano Sarmento Cabral 



\subsection{Abstract}

In complex tropical forests many different tree species compete for resources in 3D space. To understand the processes driving tree growth and forest dynamics, we developed a long-term dynamic forest stand model simulating trees as detailed 3D functional-structural tree models (FSTMs). The accurate representation of tree structure in FSTMs allows detailed simulations of within-tree processes and interactions with the environment, making such models suitable tools to explore how 3D tree growth emerges from low-level processes. In addition, complex interactions between individual trees at the metamer level can be simulated when integrating FSTMs in forest models, which goes beyond modern forest stand models.

High species numbers and diverse ecological strategies of tropical trees pose a particular challenge for dynamic forest models. We expected a correlation between leaf economic and life history traits. Important leaf economic traits (e.g. leaf life span, photosynthetic capacity) co-vary strongly and variation is largely explained by a single principle axis - the leaf economics spectrum (LES). Consequently, tree species in our model are characterized by a set of traits corresponding to a specific position on the LES. Applying the principles of the pipe model theory, light-driven carbon assimilation and within-tree carbon allocation are coupled, i.e. 3D tree growth is essentially driven by leaf scale processes. To investigate the suitability of this approach, we compared a large number of emergent patterns at the tree level as well as at the forest level in a pattern-oriented modeling framework.

We found that a species' set of economic leaf traits determined the maximum height and age of a tree, as well as its size-dependent growth rate and shade tolerance, indicating a fundamental impact of leaf traits on the life history growth patterns of trees. In addition, many ecological patterns at the forest level (e.g. above-ground biomass, basal area, stem number, net-primary production and leaf area index) were reproduced, further validating the model and indicating that our model adequately simulates structurally realistic forests. The modelling approach presented here paves the way for further model-based analyses of 3D forest dynamics, or model-based studies of canopy-dwelling organisms requiring a detailed representation of forest structures and their dynamics.

\subsection{Introduction}

Tropical forests provide valuable social, ecological and economical services to society and play an important role in the global carbon cycle (Malhi \& Grace 2000; Hassan, Scholes \& Ash 2005). They are the most species-rich ecosystems harboring more than half of the species on earth (Heywood \& Watson 1995). In addition to the large number of tree species (ter Steege et al. 


\section{Functional-structural forest model}

2013), there are even more plant and animal species that directly or indirectly depend on the structure, resources and shelter provided by complex forest canopies (Erwin 1988). Almost 9\% of all vascular plant species, for instance, live as epiphytes on trees, predominantly in subtropical or tropical regions (Zotz 2013). Ongoing deforestation and potential adverse effects of climate change thus pose a threat to all species associated with tropical forest systems (Wright 2005). To assess the impact of a changing environment on tropical biodiversity, we thus need to understand how these forests function and respond to those changes, but also how associated species respond to changing forest dynamics.

There are a number of dynamic forest models available to predict future changes of tropical forests and/or to analyze their ecosystem functions. These models differ substantially in the level of detail and temporal and spatial resolution. Among these models, dynamic global vegetation models focus on large-scale predictions of vegetation dynamics and carbon cycles, but commonly use very simplified representations of forest structure (e.g. Cramer et al. 2001; Purves \& Pacala 2008). At small to medium scales $(<1$ ha to $>100 \mathrm{~km}$ ), forest gap models and forest landscape model are applied to simulate forest dynamics and tree species composition (reviewed in Bugmann 2001). Such models represent forest structure in more detail by including stems and crowns of individual trees or cohorts, they consider within-canopy light attenuation and simulate growth of and competition among different species or functional types (Köhler \& Huth 1998; Tietjen \& Huth 2006). An even more detailed simulation of tree structure is given in functional-structural tree models (FSTMs), in which trees are represented in 3D space by interconnected structural and functional units, such as branch segments, leaves, or reproductive organs (Godin \& Sinoquet 2005; Sievänen et al. 2014). These 'virtual tree' models allow to model complex, mechanistic interactions between tree architecture and physiological processes, for instance the lightdependent within-tree carbon acquisition and allocation at the meristem level in dynamically growing trees (Sterck et al. 2005; Fourcaud et al. 2008). FSTMs are thus suitable tools to explore and deepen our understanding of structural tree growth, and a natural next step would be the integration of FSTMs in forest stand models. In such models, interactions among trees, microclimatic changes and branch dynamics could be simulated in detail at the forest level. Such detailed simulations of forest structures and their dynamics would also be useful for model-based studies of canopy-dwelling plants and animals (Sarmento Cabral et al. 2015). However, only few attempts have been made to couple FSTMs with forest stand models, and these studies focused on growth of even-age monocultures over a limited time frame (Feng et al. 2011; Guillemot et al. 2014). So far, there is no long-term fully-dynamic stand model based on FSTMs including demographic processes beyond vegetative growth.

Developing a fully-dynamic 3D tropical stand model is computationally and conceptually challenging. On the one hand, FSTMs are computer intensive due to their complexity, and stand- 
scale FSTMs thus require efficient modelling techniques allowing detailed simulations while keeping the simulation time reasonably low. On the other hand, tropical forests pose particular challenges to dynamic forest models due to their large number of tree species (ter Steege et al. 2013; Slik et al. 2015). In contrast to temperate forests, where the low number of well-studied tree species allow models to be parameterized at the species level, alternative approaches are required. In individual-based tropical forest models, distinct functional groups aggregating tree species with similar characteristics are thus usually used (e.g. Köhler \& Huth 1998; Tietjen \& Huth 2006). In the simplest case, only shade-intolerant pioneers and shade-tolerant climax species are distinguished (Swaine \& Whitmore 1988), but a classification into more groups has also been proposed (Gourlet-Fleury et al. 2005; Chazdon et al. 2010). While functional group approaches are often useful, they still are a simplification of the continuum from fast growing, short-lived pioneer to slow growing, long-lived shade-tolerant species (Denslow 1987; Wright et al. 2003b). Similar trade-offs between growth and mortality have also been observed at the leaf scale by Wright et al. (2004), who found that many leaf traits co-vary strongly and that this variation is largely explained by a single principle axis - the leaf economics spectrum (LES). This spectrum runs from leaves with high photosynthetic capacities but low life spans at the one end to leaves with low photosynthetic capacities but long life spans at the other end. Hence, a relationship between the leaf traits and the whole-tree performance can be assumed, and significant relationships were indeed observed for many tropical tree species (Sterck, Poorter \& Schieving 2006; Poorter \& Bongers 2006). A trait-based approach should thus be a promising way to integrate the different life history strategies of trees into a forest model, obliterating the use of $a$ priori functional groups. However, we are not aware of any study in which 3D growth over a tree's entire life span is modelled as an emergent property of the tree's set of traits.

In this study, we present a dynamic forest stand model in which each trees is represented as a 3D FSTM. This model was developed to simulate the long-term forest dynamics (500-1000 years) at the plot scale ( $\sim 1 \mathrm{ha}$ ) with a high degree of detail. Branches are considered up to the second order and leaf biomass development is modelled at a resolution of $1 \mathrm{~m}^{3}$, which allows detailed simulations of competition for light and space. Tree species are characterized by a set of leaf traits under consideration of the between-traits trade-offs and correlations (LES; Wright et al. 2004). Using the principles of the pipe model theory (Shinozaki et al. 1964), the light-driven carbon assimilation and the within-tree carbon allocation are coupled, i.e. the leaf trade-offs are scaled to whole-tree growth. We hypothesize that this trait-based approach captures essential life history variations between different species/functional groups with regard to their growth, survival, and light demand. In addition, we assume that the long-term dynamics of natural tropical forests can be reproduced by coupling the FSTM with a forest stand model, in which the key demographic processes and between-tree competition are simulated in a simplistic manner (e.g. only 


\section{Functional-structural forest model}

considering competition for light and space, neutral regeneration). Such a model can, on the one hand, improve our understanding of how low-level processes (leaf scale) influence pattern at higher hierarchical levels (tree and forest scale). On the other hand, by providing the 3D forest structure and dynamics, this model can form the basis for future modelling studies of canopy dwelling organisms, e.g. vascular epiphytes (see chapter 5).

\subsection{Materials and methods}

In this section, we provide the model description of the bottom-up functional-structural forest model (FSFM) and details on the parameterization and validation process.

\subsubsection{Model description}

The model description follows the ODD (Overview, Design concepts, Details) protocol, which was proposed as a standard protocol to communicate agent-based models or large, complex models (Grimm et al. 2006, 2010).

\subsubsection{Purpose}

The FSFM serves two main purposes. On the one hand, it was developed to study the relationship between leaf trait trade-offs and life-history variation in trees. Ontogenetic growth patterns, maximum height and life-span, as well as the light-dependent growth behavior in our model emerge from the tree's traits, and the model thus allows to compare simulated structural tree growth with observations and theoretical expectations. On the other hand, it was developed to simulate the long-term dynamics of forest stands at a high level of detail. By combining the traitbased tree growth model with a simple demographic model, the suitability of our approach can also be evaluated at the forest level. Our forest model increases the understanding of bottom-up mechanisms controlling forest dynamics, and in addition, it is useful for follow-up model studies on canopy-dwelling organisms, which require a detailed 3D representation of forest structure and dynamics.

\subsubsection{Entities, state variables and scales}

This FSFM simulates establishment, growth and mortality of virtual 3D trees at the plot level. The spatial and temporal scale of the model can by defined by the user. Here, we simulated forest stands between 0.25 and 1 hectare over 500 to 1000 years in annual time steps. The vertical extension of the model space is associated with typical maximum tree heights and usually ranges 
between 50 and $60 \mathrm{~m}$. The entire 3D model space is divided into a regular 3D grid consisting of cubic voxels with a side length of $1 \mathrm{~m}$ (Fig. 4.1a). This grid defines the spatial resolution of both light and leaf area/biomass distribution. Light is the main driver of tree growth and the light intensity is calculated for all voxels based on the 3D distribution of leaf area.

This model comprises three hierarchical levels: tree components, individual trees, and the forest stand. Tree components are trunks, branches, apical meristems and leaf compartments (Fig. 4.1b). Each tree consists of one erect trunk described by length and diameter. Attached to the trunk are branches up to the second order. Branches are defined at two different scales. At the coarse scale, branches are described by their total length and diameter, while at the fine scale branches are described as a collection of topologically connected smaller branch segments (this multiscale approach was chosen to optimize both model speed and visual aspects; see section 4.3.1.7 for more details). Located at the end of each trunk or branch, apical meristems sense the local environment and are able to control primary growth. Leaf compartments are connected with second order branches and are conceptualized as aggregations of leaves within the cubic voxels. Besides leaf biomass and area, leaf compartments also comprise the active pipes, i.e. the sapwood, connecting the leaves with the roots to support leaf functioning. This means that leaf compartments form leaf-pipe elements in accordance with the pipe model theory (Shinozaki et al. 1964; Fig 4.1b). Each tree component is characterized by a set of state variables including its 3D position (Table 4.1). In addition to the absolute 3D position, the topological position within the tree of each tree component is also tracked throughout the simulation. Based on this information, the 3D structure and internal organization of each tree can be deduced (Fig. 4.1c). Structural tree growth is thus the result of addition, removal, and changes in tree components. Aggregation over all trees in the community yields the 3D forest structure and dynamics.

Structural tree growth is driven by the distribution of light and the functional and structural traits of trees, which can be understood as intrinsic properties (Table 4.1). While the functional traits regulate tree carbon balance depending on the light conditions, the structural traits can be regarded as inherent architectural model defining the tree's structural organization. This includes, for instance, branching angles or average internode lengths (see submodel structural growth in section 4.3.1.7 for more details). Growth and performance of individual trees are thus closely associated with their combination of traits. Some functional trait combinations allow effective carbon assimilation under low-light conditions and thus to grow and survive in the dark understory, while other trait combinations might be more favorable under high-light conditions. Consequently, forest dynamics results from structural growth of individual trees with different traits interacting and competing for space and light, whose distribution, in turn, is influenced by the forest structure (Fig. 4.1d). 
(a)

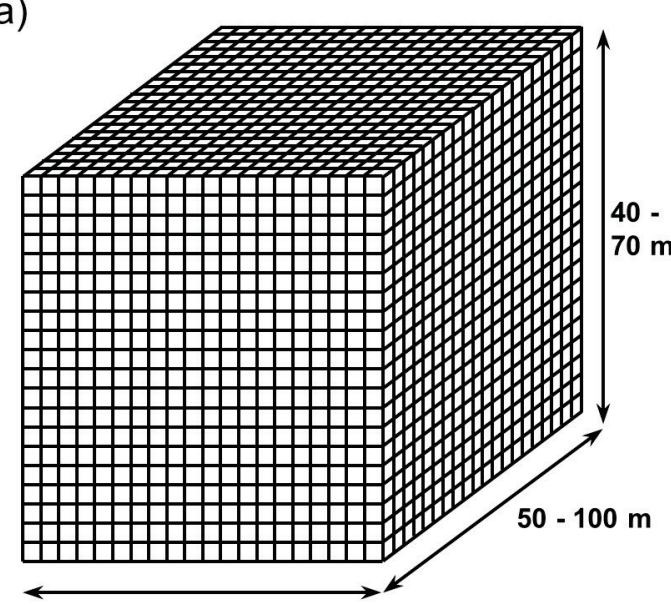

$50-100 m$

(c)

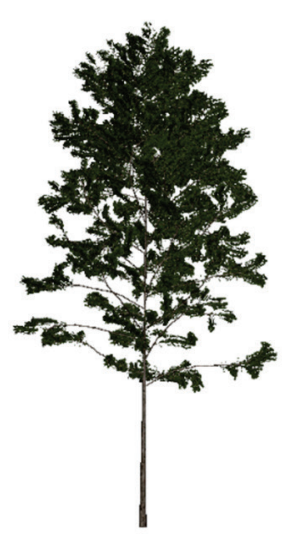

(d) (b)

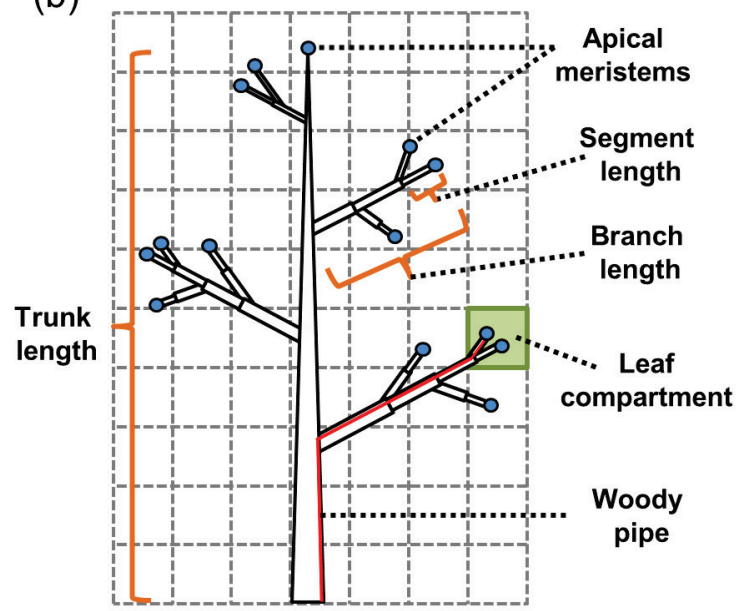

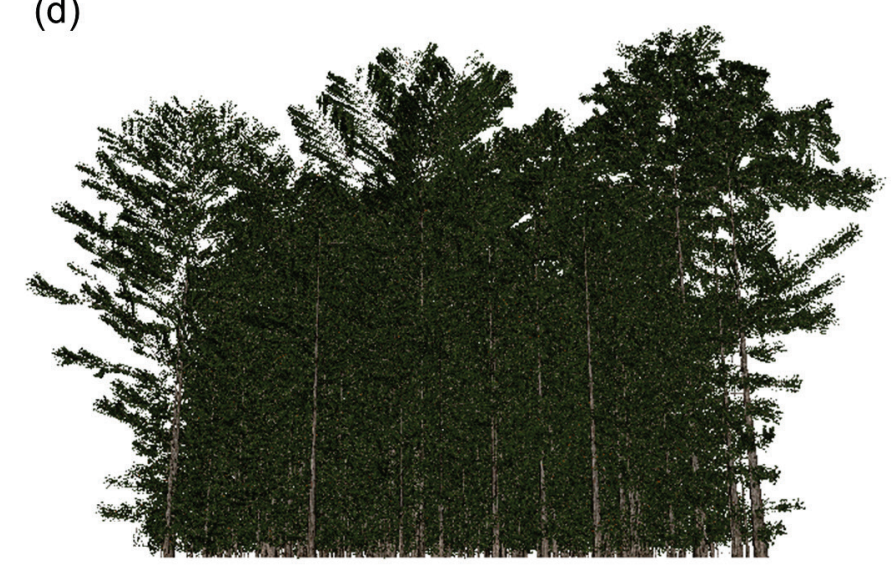

Figure 4.1. State variables, scales and visualization. (a) 3D model space. The extent of the model space can be defined by the user. The model space is a $3 \mathrm{D}$ grid that is subdivided into cubic voxels with a volume of $1 \mathrm{~m}^{3}$ containing the information about local leaf biomass and area, as well as, light intensity. (b) Overview of tree components (trunks, branches, apical meristems and leaf compartments). Trees consist of a trunk and branches up to the second order which are terminated by an apical meristem. Leaf compartments describe the leaf biomass and area within a voxel attached to a specific section of a second order branch, as well as the woody pipes connected to these leaves. The length of the pipe system depends on the within-tree position. One leaf compartment (green square) and its woody pipe (red line) are exemplified. (c) 3D tree visualization. Tree structures are visualized based on the state variables and topology of each tree. Several visualization options are integrated in this model (section 4.3.2). Here, the leaf biomass in the leaf compartments is displayed by spatial objects imitating 'real' leaves. (d) 3D forest visualization. The forest structure can be displayed in this model, which allows visual inspections and comparisons with real forests. 
Table 4.1. State variables, functional and structural traits of the FSFM. Each tree component (trunk, branch, leaf compartment, apical meristem) is characterized by a set of state variables. The functional and structural traits describe the intrinsic properties of each tree species, and the value ranges of these traits can be defined by the user. These trait ranges are thus among the model parameters that are used to calibrate and validate the model. Empirical correlations between leaf traits (Wright et al. 2004) are considered in this model (Table 4.2).

\begin{tabular}{|c|c|c|c|}
\hline Symbol & Description & Unit & Type \\
\hline$A_{B}$ & Cross-sectional area of branch & $\mathrm{cm}^{2}$ & State variable \\
\hline$A_{\mathrm{L}}$ & Leaf area in leaf compartment & $\mathrm{cm}^{2}$ & State variable \\
\hline$A_{\text {LProd }}$ & Total leaf area produced in leaf compartment & $\mathrm{cm}^{2}$ & State variable \\
\hline$A_{S}$ & Cross-sectional area of branch segment & $\mathrm{cm}^{2}$ & State variable \\
\hline$A_{T}$ & Cross-sectional area of trunk & $\mathrm{cm}^{2}$ & State variable \\
\hline$B_{\mathrm{L}}$ & Leaf biomass in leaf compartment & $\mathrm{g}$ & State variable \\
\hline$D_{\text {B }}$ & Diameter of branch & $\mathrm{cm}$ & State variable \\
\hline$D_{\mathrm{S}}$ & Diameter of branch segment & $\mathrm{cm}$ & State variable \\
\hline$D_{T}$ & Diameter of trunk & $\mathrm{cm}$ & State variable \\
\hline$I_{\mathrm{M}}$ & Light conditions at apical meristem & $\mu \mathrm{mol} \mathrm{m} \mathrm{m}^{-2} \mathrm{~s}^{-1}$ & State variable \\
\hline$L_{\mathrm{B}}$ & Length of branch & $\mathrm{cm}$ & State variable \\
\hline$L_{\mathrm{P}}$ & Pipe length of leaf compartment (corrected after apical control) & $\mathrm{cm}$ & State variable \\
\hline$L_{\mathrm{PS}}$ & Pipe length of leaf compartment & $\mathrm{cm}$ & State variable \\
\hline$L_{\mathrm{S}}$ & Length of branch segment & $\mathrm{cm}$ & State variable \\
\hline$L_{T}$ & Length of trunk & $\mathrm{cm}$ & State variable \\
\hline$O_{\mathrm{B}}$ & Branch order & - & State variable \\
\hline$P_{\mathrm{BEnd}} \mathrm{XYZ}$ & End position of branch (in $\mathrm{X}, \mathrm{Y}$ and $\mathrm{Z}$ direction) & $\mathrm{cm}$ & State variable \\
\hline$P_{\text {BStart }} \mathrm{XYZ}$ & Start position of branch (in $\mathrm{X}, \mathrm{Y}$ and $\mathrm{Z}$ direction) & $\mathrm{cm}$ & State variable \\
\hline$P_{\mathrm{LC}}^{\mathrm{XYZ}}$ & Position of leaf compartment (in $\mathrm{X}, \mathrm{Y}$ and $\mathrm{Z}$ direction) & $\mathrm{cm}$ & State variable \\
\hline$P_{\mathrm{M}}^{\mathrm{XYZ}}$ & Position of apical meristem (in $\mathrm{X}, \mathrm{Y}$ and $\mathrm{Z}$ direction) & $\mathrm{cm}$ & State variable \\
\hline$P_{\text {SEnd }} \mathrm{XYZ}$ & End position of branch segment (in $\mathrm{X}, \mathrm{Y}$ and $\mathrm{Z}$ direction) & $\mathrm{cm}$ & State variable \\
\hline$P_{\text {SStart }} \mathrm{XYZ}$ & Start position of branch segment (in $\mathrm{X}, \mathrm{Y}$ and $\mathrm{Z}$ direction) & $\mathrm{cm}$ & State variable \\
\hline$P_{\mathrm{T}}^{\mathrm{XY}}$ & Position of trunk (in $\mathrm{X}$ and $\mathrm{Y}$ direction) & $\mathrm{cm}$ & State variable \\
\hline$G_{\max }$ & Maximum gross photosynthetic rate & $\mathrm{g} \mathrm{g}^{-1} \mathrm{~d}^{-1}$ & Functional trait \\
\hline$k$ & Light intensity at which the gross photosynthetic rate is half maximal & $\mu \mathrm{mol} \mathrm{m} \mathrm{m}^{-2} \mathrm{~s}^{-1}$ & Functional trait \\
\hline$L L$ & Leaf lifespan & $\mathrm{d}$ & Functional trait \\
\hline$N_{\text {mass }}$ & Nitrogen concentration & $\%$ & Functional trait \\
\hline$R_{\mathrm{L}}$ & Leaf respiration rate & $\mathrm{g} \mathrm{g}^{-1} \mathrm{~d}^{-1}$ & Functional trait \\
\hline$S L A$ & Specific leaf area & $\mathrm{cm}^{2} \mathrm{~g}^{-1}$ & Functional trait \\
\hline$\rho_{\mathrm{W}}$ & Wood density & $\mathrm{g} \mathrm{cm}^{-3}$ & Functional trait \\
\hline$A_{\text {LProd }}$ & Maximal leaf area production per leaf compartment & $\mathrm{cm}^{2}$ & Structural trait \\
\hline$I_{\mathrm{T}}$ & Light intensity threshold regulating apical dominance of SAM & $\mu \mathrm{mol} \mathrm{m} \mathrm{m}^{-2} \mathrm{~s}^{-1}$ & Structural trait \\
\hline$k_{\text {int }}$ & factor controlling the increase in internode length & - & Structural trait \\
\hline$L D_{\mathrm{B}}$ & Length-diameter ratio of branches & $\mathrm{m} \mathrm{cm}^{-1}$ & Structural trait \\
\hline$L D_{\mathrm{T}}$ & Length-diameter ratio of trunks & $\mathrm{m} \mathrm{cm}^{-1}$ & Structural trait \\
\hline$L_{\mathrm{IBMax}}$ & Maximal internode length of branches & $\mathrm{cm}$ & Structural trait \\
\hline$L_{\text {IBMin }}$ & Minimal internode length of branches & $\mathrm{cm}$ & Structural trait \\
\hline$L_{\text {ITMax }}$ & Maximal internode length of trunk & $\mathrm{cm}$ & Structural trait \\
\hline$L_{\text {ITMin }}$ & Minimal internode length of trunk & $\mathrm{cm}$ & Structural trait \\
\hline$P H_{\mathrm{FO}}$ & Number of first order branches arranged in a $360^{\circ}$ circle & - & Structural trait \\
\hline$P_{\mathrm{RU}}$ & Pipe-reuse factor & - & Structural trait \\
\hline$S_{\mathrm{F}}$ & Shortening factor & - & Structural trait \\
\hline$S_{\text {Trop }}$ & Strength of tropism & - & Structural trait \\
\hline$S T_{\text {Trop }}$ & Stochasticity in tropism strength $\left(S_{\text {Trop }}\right)$; only used if Stochasticity $=1$ & $\%$ & Structural trait \\
\hline$S T_{\mathrm{Tw}}$ & Stochasticity in branch growth; only used if Stochasticity=1 & $\%$ & Structural trait \\
\hline$S T_{\mathrm{aSFO}}$ & Stochasticity in second order angle $\left(\alpha_{S F O}\right)$; only used if Stochasticity $=1$ & $\%$ & Structural trait \\
\hline$S T_{\alpha \mathrm{TFO}}$ & Stochasticity in first order angle $\left(\alpha_{T F O}\right)$; only used if Stochasticity $=1$ & $\%$ & Structural trait \\
\hline$S T_{\alpha \mathrm{TSO}}$ & Stochasticity in first order angle $\left(\alpha_{T S O}\right)$; only used if Stochasticity $=1$ & $\%$ & Structural trait \\
\hline$\alpha_{S F O}$ & Angle between first order branches and trunk from side view & $\circ$ & Structural trait \\
\hline$\alpha_{T F O}$ & Angle between first order branches from top view & $\circ$ & Structural trait \\
\hline$\alpha_{T S O}$ & Angle between second order branches and first order branches from top & $\circ$ & Structural trait \\
\hline$B_{D}$ & Maximum relative increase in height growth when the $I_{\mathrm{M}}<I_{\mathrm{T}}$ & - & Structural trait \\
\hline$B_{S}$ & Shape parameter regulating apical dominance of trunk apical meristem & - & Structural trait \\
\hline
\end{tabular}




\section{Functional-structural forest model}

\subsubsection{Process overview and scheduling}

At the beginning of each simulation, a species pool with a defined number of tree species is generated. Each species has a unique identifier and is characterized by a set of functional and structural traits (Table 4.1). We assume that the structural traits are uncorrelated, and these traits are randomly selected from user-defined ranges. In contrast, for functional leaf traits, we consider the strong between-trait correlations (Wright et al. 2004; Marino, Aqil \& Shipley 2010; more details in section 4.3.1.5). After this initialization, light distribution, tree establishment, tree growth, and tree mortality are simulated successively in annual time steps (Fig. 4.2).

The 3D distribution of light intensity is calculated via the Lambert-Beer light extinction law based on the distribution of leaf area. Subsequently, the establishment of tree seedlings is simulated as a neutral process, i.e. the forest floor is regarded as seed bank containing equal numbers of seeds of all tree species. Depending on an average area-based establishment rate, a certain number of new seedlings is initialized at random positions within the model area. Each seedling is randomly assigned to a species from the species pool. After this neutral germination, seedlings of species with unsuitable traits may die immediately within the current time step due to carbon starvation.

Tree growth is simulated in three subsequent submodels: i) apical control/dominance, ii) carbon balance, iii) structural growth (Fig. 4.2): i) controlled by hormones, carbon allocation to apical meristem can either be inhibited (apical control) or intensified (apical dominance; Wilson 2000). These processes control how much of the carbon assimilated by photosynthesis is invested into primary growth of branches and the trunk. In this model, apical control is simulated for branches. Branches inhibit carbon allocation to primary growth when branch apical meristems are either deeply shaded, i.e. if the carbon balance under the given light conditions at the meristem is negative, or when branches from other trees grow in the immediate vicinity. By this, competition for light and space is simulated at the branch level. In contrast, apical dominance is simulated for trunks, i.e. carbon allocation to trunk apical meristems is intensified under shade as a mechanism to quickly reach higher, potentially less shaded zones (Poorter 1999; Poorter et al. 2011). Naturally, by influencing the within-tree carbon allocation, the processes of apical dominance/control affect local carbon balance, which is simulated in the second step. ii) Local carbon balance corresponds to the carbon balance at the level of leaf compartments. Apart from the carbon assimilated by leaf compartments and allocated to primary growth, leaf compartments act independently from each other and directly respond to the local light conditions. This means that no carbon flow is assumed between leaf compartments, and thus the assimilated carbon is locally reinvested into biomass of new leaves and connected pipes. Leaves are the photosynthetically active organs, but both leaves and pipes incur respiration costs. The annual leaf biomass production and the annual change in leaf biomass in each leaf compartment are thus 
important results of the carbon balance submodel. Due to the leaf-pipe connection, these results are directly linked to secondary growth of branches and trunks. iii) Structural growth is an immediate result of the carbon balance submodel. It comprises secondary, but also primary growth, which, in turn, is calculated based on secondary growth using species-specific allometric relationships between height and diameter. Primary growth causes the establishment of new apical branch segments and often new lateral branch segments, which might be associated with new leaf compartments and apical meristems. In addition, trees may also shed branches, for instance after losing all photosynthetically active leaf compartments, and this is simulated in the final step of the structural growth submodel.

At the end of each simulation step, tree mortality is simulated. Trees die due to carbon starvation when they have lost all leaf compartments. In addition, we integrated a biomass-dependent mortality rate according to metabolic theory of ecology (Brown et al. 2004). This rate accounts for processes which are not explicitly simulated (e.g. herbivory, pathogens) and assumes that the chance of survival increases non-linearly with total tree biomass. Gap dynamics are also an important mechanism in tropical forests (Brokaw 1985). Falling dead trees may kill surrounding trees and create gaps, and thus we also integrated the option to model this.

After each of the processes illustrated in Fig. 4.2 the state variables of all trees components are updated synchronously.

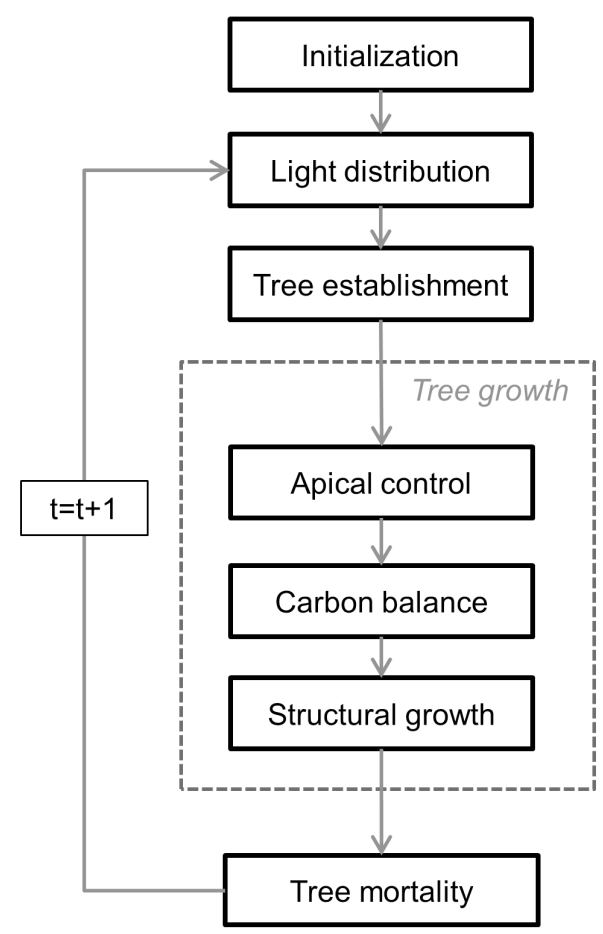

Figure 4.2. Flowchart of the forest model. After initialization, light distribution, tree establishment, growth and mortality are simulated consecutively in each annual time step. Tree growth is the most complex process and thus split into three submodels: apical control, carbon balance and structural growth. Details on all submodels are provided in section 4.3.1.7. 


\section{Functional-structural forest model}

\subsubsection{Design concepts}

\section{Basic principles}

Carbon assimilation and allocation are the key processes in functional-structural tree and forest models. In our model, these processes are simulated based on the principles of the leaf economics spectrum (Wright et al. 2004), the pipe model theory (Shinozaki et al. 1964), and the principles of module autonomy (Sprugel, Hinckley \& Schaap 1991). The LES quantifies relationships between crucial leaf economic traits, such as $S L A$, leaf lifespan or mass-based photosynthetic capacity. These leaf traits co-vary strongly and, in multidimensional trait space, the vast majority of variation is explained by a single principle axis (Wright et al. 2004). This axis can be considered as spectrum, ranging from leaves with low $S L A$ values, low photosynthetic capacities, and respiration rates, but long leaf lifespans, to leaves with high $S L A$ values, high photosynthetic capacities and respiration rates, but short leaf lifespans. The position on this spectrum thus has a direct influence on potential carbon assimilation and re-allocation at the leaf level. Furthermore, Marino, Aqil \& Shipley (2010) observed that not only photosynthetic capacity, but also entire photosynthetic light-response curves can be predicted from the leaf traits of the LES. With this information, the carbon balance at the leaf level under varying light conditions can thus be simulated based on the specific leaf trait combination described by the position at the LES. However, the carbon assimilated by the leaves may be allocated among different tree parts, i.e. it may be invested into new leaves or branches at different within-tree positions. In this model, the within-tree carbon allocation is based mainly on the principles of module autonomy (Sprugel et al. 1991) and the pipe model theory (Shinozaki et al. 1964). The principles of module autonomy state that different parts of the tree may be regarded as autonomous modules whose carbon balance is independent of that of other modules. In our model, leaf compartments are these autonomous modules, which assimilate carbon based on their leaf traits and the local light intensity, and re-invest assimilates locally (Only a small exception from this rule is allowed in our model, as a small part of the assimilates in each leaf compartment is allocated for primary growth of the corresponding branch. There is, however, no carbon flow among leaf compartments). Local re-investment means investments in leaf biomass within the leaf compartment which, however, are coupled with investments in connected woody pipes. In other words, for each new unit of leaf biomass an equivalent unit of pipes connecting leaves and roots has to be established, whereby the within-tree position of a leaf compartment determines the carbon costs for the pipes. New active pipes form the sapwood, which is equivalent to secondary or primary growth of branches and the trunk. By considering all leaf compartments of a tree, the whole-tree carbon balance and resulting structural growth can thus sufficiently be simulated based on the principles described above. 


\section{Emergence}

Each tree is characterized by a set of traits, and structural tree growth, i.e. development, addition and removal of tree components, is a direct result of the interplay between these traits and light conditions. Hence, tree growth and tree mortality emerge from the traits of a tree. Some trait combinations might be unsuitable under low-light conditions and thus lead to carbon-based starvation. However, even under optimal conditions, each tree in the model will inevitably die at some point in time because it has lost all its photosynthetically-active parts. This is because the maximum height of each tree also emerges from its traits. When a tree grows close to its maximum height it will enter senescence, which is characterized by the reduction of active meristems ultimately leading to the loss of all leaves (for more details see submodel structural growth in in section 4.3.1.7). Consequently, all crucial processes over the entire life cycle, as well as life expectancy itself, emerge directly from the functional and structural traits characterizing an individual tree.

While forest structure is the result of the growth of interacting and competing trees with different traits, community dynamics emerges from the trait-based mechanism at the tree level, as well as from tree establishment and additional source of tree mortality. The establishment rate defines how many new recruits enter the community, and different tree mortality rates are integrated to account for additional sources of mortality not captured by the FSTM.

\section{Adaptation/Sensing}

In this model, the interplay between the invariable functional and structural traits of trees and the dynamic environment determines their growth, but trees cannot adapt their traits to the environment. In reality, trait adaptations in response to environmental conditions may be observed within individuals. For instance, traits of sun and shade leaves might differ (Rozendaal et al. 2006; Markesteijn et al. 2007). However, in this model approach, we were more interested in interspecific trait differences than in trait differences within individuals

While adaptation and fitness-seeking of individual trees is not modelled explicitly at the trait level, we integrated two mechanisms controlling the primary growth of branches and trunks in dependence on the light conditions. The apical branch and trunk meristems sense their environment and, on this basis, either inhibit or intensify carbon allocation to the apical meristems. For branches, carbon allocation and thus primary growth is inhibited if the apical meristem senses insufficient light conditions or branches from neighboring trees in the immediate vicinity. This prevents carbon investments in tree parts with potentially low photosynthetic revenue. For trunks, primary growth is intensified under shade to reach higher, potentially less 


\section{Functional-structural forest model}

shaded zones faster. These apical control mechanisms can be understood as adaptation to the environment which may improve the fitness of the individuals.

\section{Interaction}

Both indirect and direct interactions among individuals are simulated. As the 3D light distribution is determined by the 3D leaf distribution in the community, competition for light is modelled as indirect interaction among the individuals. In contrast, crown development is directly influenced by competition for space between neighboring trees, because if trees sense tree components from neighboring trees in their immediate vicinity, they stop carbon allocation to this area. In addition, we integrated an option to simulate a direct feedback of falling trees on the mortality of neighboring trees, i.e. gap formation.

\section{Stochasticity}

The species pool containing the trait information of all local tree species is randomly drawn from user-defined ranges or estimated based on established between-trait correlations according to the LES (Wright et al. 2004). Tree establishment and mortality are also stochastic. The number of new seedlings at each time step can either be defined as a fixed value or as a range, from which the actual number is randomly chosen. Each new seedling is randomly distributed over the model area and a random species identity is assigned to it. Apart from trait-based cause of mortality (e.g. carbon starvation), we additionally integrated stochastic mortality: based on its current biomass the mortality probability for each individual is estimated, and the decision whether to live or die is based on randomly drawn numbers. This additional mortality term covers sources of mortality which are not captured by the FSTM, such as infections by pathogens or excessive herbivory. Furthermore, if gap formation is simulated, trees die with a certain probability if large trees die nearby.

While the carbon balance of each individual tree is deterministic, the user can define if structural growth should be deterministic or stochastic. Deterministic structural growth means that trees strictly follow their structural model defined by their traits, i.e. branching angles are invariable and branches grow straight. Alternatively, stochastic structural growth can be switched on. In this case, individuals may randomly deviate from their regular structural growth within defined ranges and, as a consequence, branches grow irregularly. Choosing the stochastic structural growth model generates trees with a more natural and realistic appearance. 


\section{Observation}

Emergent results can be monitored and saved at any hierarchical level (community, individuals, and tree components) at each time step from an omniscient perspective. Results at the community level include both stand variables and rates, such as the total above-ground biomass, the number of trees, total mortality rates or the net primary production. At the level of individual trees, aggregated variable such as the total tree height, crown width or height at first branching are recorded, while at the lowest hierarchical level, the state variables of all tree components are monitored (Table 4.1). As the amount of data at the low hierarchical levels can be enormous (a 1 ha plot may consist of several million tree components), we integrated the opportunity to select the time intervals at which the different model results are saved. Additionally, the graphical display of the simulated forest can be saved at each time step. More details on model outputs and options for customization are provided after the model description.

\subsubsection{Initialization}

At the beginning of each simulation, a 3D grid space is initialized, whose spatial extent is defined by the parameters $\operatorname{Max} X, \operatorname{Max} Y, \operatorname{Max} Z$ and $L_{\mathrm{Cor}}$. MaxX and $\operatorname{Max} Y$ define the core area in which trees can root, while $L_{\text {Cor }}$ defines the width of the corridor surrounding the core area in which trees may expand their crowns (Fig. 4.3). Cubic voxels of the grid space have a side length of $L_{\mathrm{V}}$ and are clustered as 3D matrix (Fig. 4.1a). As the model space is initially empty, the total leaf area in all voxels is zero and thus the light intensity is at the global maximum $I_{\max }$.

In addition, the species pool containing the trait information of $n_{\text {Spec }}$ species is initialized. For this purpose, the values of all structural traits for all species (Table 4.1) are randomly drawn from uniform distributions, whose minimum and maximum values are user-defined. In contrast, only two main functional traits characterizing the wood density $\left(\rho_{\mathrm{W}}\right)$ and the specific leaf area $(S L A)$ are randomly drawn from uniform distributions with natural trait ranges. The additional functional traits are estimated based on correlations with these traits (Table 4.2) These correlations account for inevitable trade-offs and thus the sets of species-specific leaf traits represent natural trait combinations. 


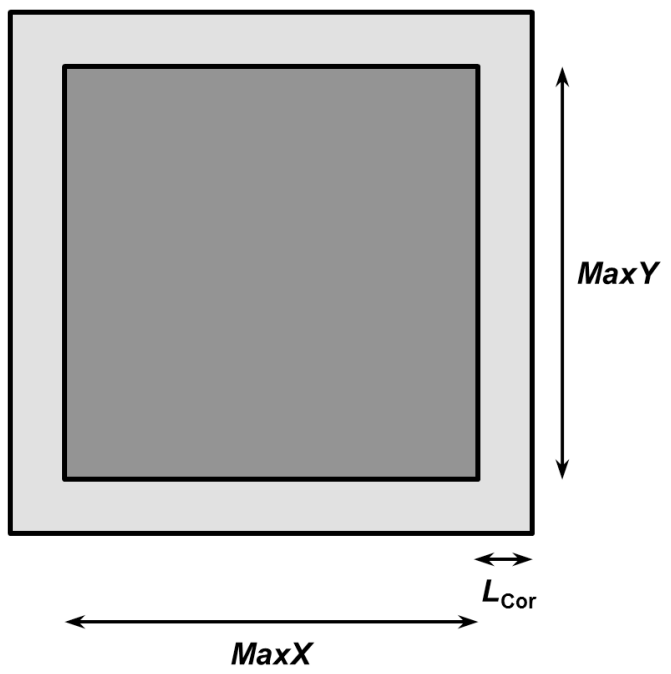

Figure 4.3. Top view on the model area. The core area in which trees can root is depicted in dark grey, the corridor in which trees may expand their crowns in light grey.

Table 4.2. Correlations between functional traits. The wood density $\rho \mathrm{w}$ and the specific leaf area $S L A$ are the only traits which are freely chosen from defined ranges for each species. The leaf life span $L L$ and foliar nitrogen concentration $N_{\text {mass }}$ are determined based on correlations with SLA following Wright et al. (2004). $R_{\mathrm{L}}, G_{\max }$ and $k$ are parameters of a hyperbolic Michaelis-Menten function determining the light response. Marino et al. (2010) found that these parameters are significantly correlated with the $S L A$ and $N_{\text {mass. }}$.

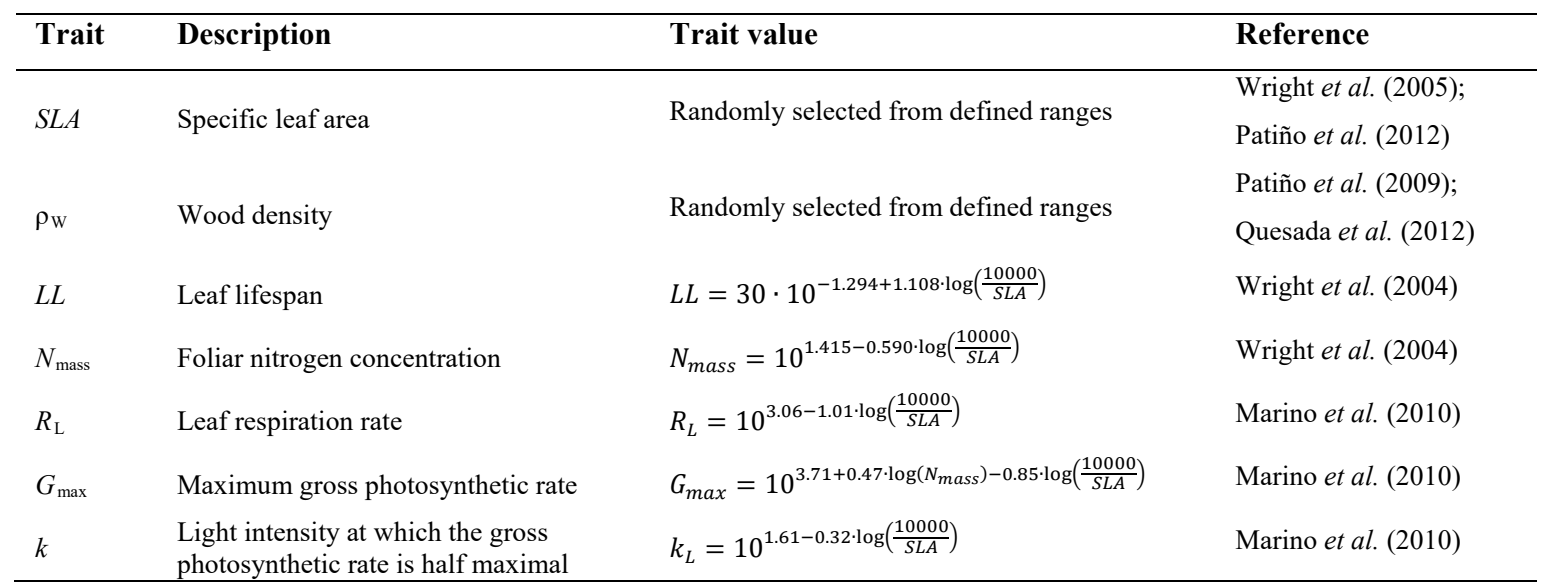

\subsubsection{Input}

The model does not use input data to represent time-varying processes. 


\subsubsection{Submodels}

In this section all submodels (light distribution, tree establishment, tree growth, tree mortality; Fig. 4.2) are described in detail and chronologically. A list of all symbols, including explanations and units, is provided as Supplementary Table C.1.

\section{Light distribution}

The 3D light environment is calculated based on the 3D leaf distribution. At first, the total leaf area in each voxel $A_{\mathrm{LTot}}{ }^{\mathrm{XYZ}}$ is estimated based on the leaf area of leaf compartments within the particular voxel $A_{\mathrm{L}}{ }^{\mathrm{XYZ}}$.

$$
A_{L T o t}{ }^{X Y Z}=\sum A_{L}^{X Y Z}
$$

Note that superscripts are used to indicate $3 \mathrm{D}$ positions. Second, based on the sum of $A_{\mathrm{LTot}}{ }^{\mathrm{XYZ}}$ in all voxels above the specified voxel, the leaf area index $L A I^{\mathrm{XYZ}}$ for each voxel is calculated.

$$
L A I^{X Y Z}=\frac{\sum_{Z}^{M a x Z} A_{L t o t}^{X Y Z}}{L_{V}{ }^{2}}
$$

where $L_{\mathrm{V}}$ is the side length of a voxel. Assuming a Lambert-Beer extinction law, the singlecolumn light intensity $I_{\mathrm{SC}}{ }^{\mathrm{XYZ}}$ is calculated based on $L A I^{\mathrm{XYZ}}$.

$$
I_{S C}{ }^{X Y Z}=I_{\max } \cdot e^{-\left(k_{L} \cdot L A I^{X Y Z}\right)}
$$

where $I_{\max }$ is the light intensity above the canopy and $k_{\mathrm{L}}$ the light extinction coefficient. This method assumes that solar radiation only penetrates directly from above and disregards additional processes like light reflection. This is an oversimplification, particularly in such heterogeneous forests as simulated here. To get a more realistic estimation of the average, effective light intensity within a voxel $I^{\mathrm{XYZ}}$, the single column light intensity $I_{\mathrm{SC}}{ }^{\mathrm{XYZ}}$ in the voxels surrounding the focal voxel in $\mathrm{x}$ and $\mathrm{y}$ direction are additionally taken into account. The number of surrounding voxels considered depends on the parameter $L R$ which defines how many rectangular rings around the focal voxel are considered (Fig. 4.4). For each considered voxel, the relative contribution $C^{\mathrm{XYZ}}$ is calculated, with $\Sigma C^{\mathrm{XYZ}}=1$. The parameter $C^{\mathrm{XYZ}}$ thus defines how strong $I_{\mathrm{SC}}{ }^{\mathrm{XYZ}}$ in each voxel contributes to $I^{\mathrm{XYZ}}$ and three different methods to calculate $C^{\mathrm{XYZ}}$ defined by the parameter LightC can be applied: either (1) all voxels or (2) all rings contribute equally, or (3) the contribution of 


\section{Functional-structural forest model}

each voxel decays exponentially with distance from the focal voxel. On this basis, $I^{\mathrm{XYZ}}$ is calculated as

$$
I^{X Y Z}=\sum_{X_{\min }}^{X_{\max }} \sum_{Y_{\min }}^{Y_{\max }} I_{S C}{ }^{X Y Z} \cdot C^{X Y Z}
$$

where $X_{\min }=X-L R$ and $X_{\max }=X+L R$ (likewise for $Y$; Fig. 4.4).

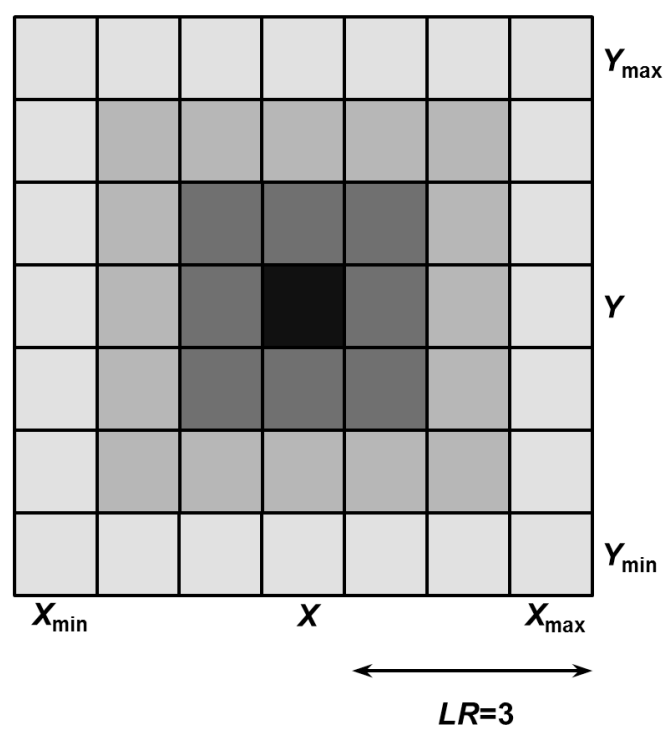

Figure 4.4. Illustration of voxels considered in calculation of effective light intensity. The light range $L R$ defines how many rectangular rings of voxels (colored in grey shades) around the focal voxel (black) are considered. For each voxel (including the focal voxel), the relative contribution of the single-column light intensity to the effective light intensity of the focal voxel is calculated based on LightC. This parameter specifies whether each voxel or each ring contributes equally, or whether the contribution of each ring decreases exponentially with distance from the focal voxel. Adjacent voxels are only considered in $\mathrm{X}$ and $\mathrm{Y}$ direction, and not in $\mathrm{Z}$ direction.

As trees can only root in the core area but expand their crowns in the corridor, the total leaf area $A_{\mathrm{LTot}}{ }^{\mathrm{XYZ}}$ decreases with distance from the forest edge, what increases the single-column light intensity $I_{\mathrm{SC}}{ }^{\mathrm{XYZ}}$ at the corridor. Consequently, the effective light intensity $I^{\mathrm{XYZ}}$ also increases in the corridor or in the core area near the corridor. Such a pattern would resemble the light distribution in small forest fragments, whose edges permit light penetration. Because we were interested in also simulating pure forest core conditions, we integrated the possibility to choose between two options: small forest fragment $(E d g e C=1)$ or forest core $(E d g e C=0)$. In the latter case, $I^{\mathrm{XYZ}}$ would not be reasonably estimated in the vicinity of the edges of the model area and thus, periodic boundary conditions are applied. This means that the forest matrix surrounding the core area is similar to the forest in the core area and thus, before applying Eq. 4.4, the single- 
column light conditions $I_{\mathrm{SC}}{ }^{\mathrm{XYZ}}$ calculated inside the core model are copied to the corridor in such a way that the conditions in the corridor resemble the conditions at the opposite side of the core area (Fig. 4.5). When the focal voxel for which $I^{\mathrm{XYZ}}$ is to be calculated is located near the edge of the entire model area (e.g. see voxel X2 in Fig. 4.5), not all adjacent voxels within the distance defined by $L R$ may exist. In this case, if periodic boundaries are specified, $I_{\mathrm{SC}}{ }^{\mathrm{XYZ}}$ for these voxels can be obtained by strictly following the principles of periodic boundaries (Fig. 4.5). If real edge conditions are specified, $I_{\mathrm{SC}}{ }^{\mathrm{XYZ}}$ for these voxels are obtained by mirroring $I_{\mathrm{SC}}{ }^{\mathrm{XYZ}}$ at the outer border.

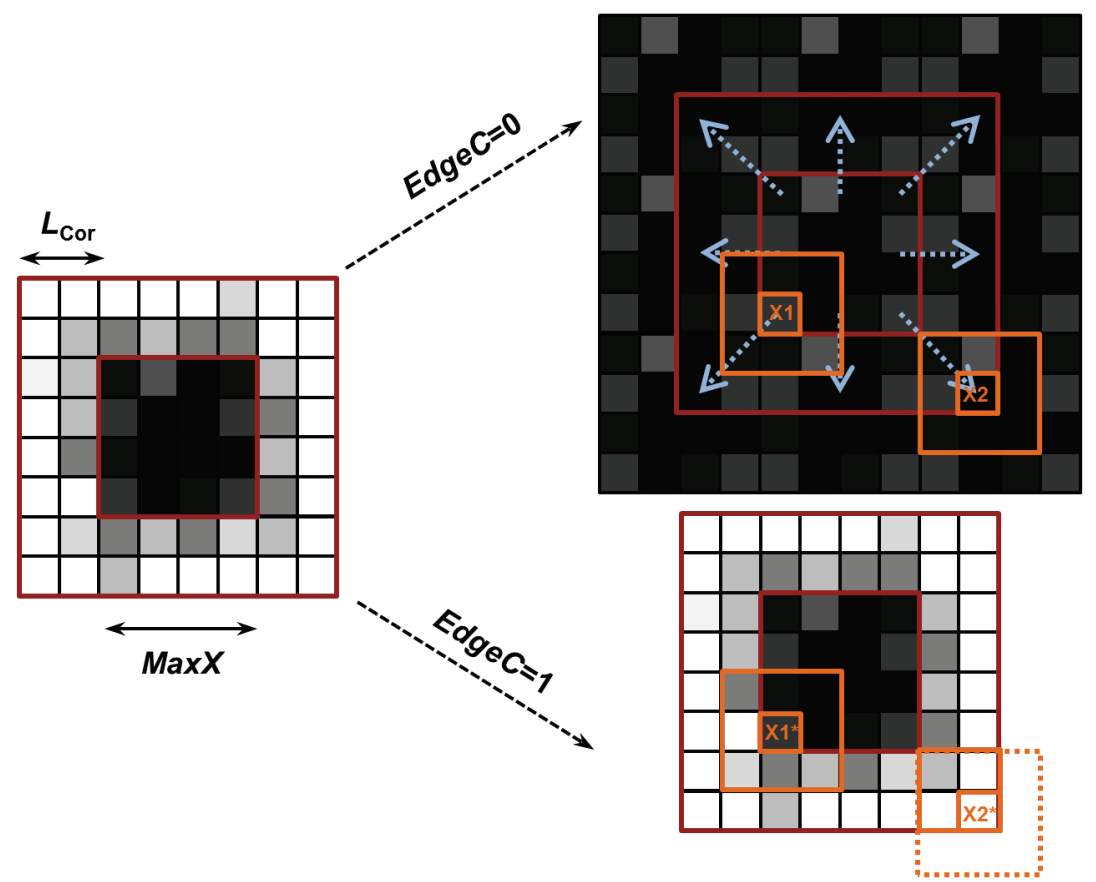

Figure 4.5. Illustration of principles applied when a small forest fragment $(E d g e C=1)$ or a forest stand within a larger forest matrix $(E d g e C=0)$ is simulated. The graph on the left side illustrates typical single-column light conditions for one horizontal voxel layer (darker colors represent lower light intensities). As tree only germinate within the core area, the single-column light conditions within the core area are typically higher compared to the corridor. If a small forest fragment $(E d g e C=1)$ is simulated, the higher light intensity values in the corridor are used to calculate the effective light intensity (bottom right panel). When, as it is the case for the voxel $\mathrm{X} 2 *$, not all surrounding voxel within $L R$ (see Fig. 4.4) exists, voxel from inside are mirrored at the outer border and considered in light calculations. If a forest stand within a larger forest $(E d g e C=0)$ is simulated, the single-column light conditions of the core area are first copied to the corridor before the effective light intensity is calculated (indicated by the blue arrows in top right panel; periodic boundary conditions).

\section{Tree establishment}

Establishment is simulated as a neutral process, i.e. all species have the same probability of establishment. New seedlings are randomly distributed over the core model area (spatial resolution: $1 \mathrm{~cm}$ ), whereby the total number of seedlings is controlled by the area-based establishment rate $n_{\text {Seed }}$. This rate can either be defined as a constant, or as a range from which 


\section{Functional-structural forest model}

the number of seedlings is randomly drawn at each time step. A randomly selected species ID from the species pool is assigned to each seedling, which is then initialized with the speciesspecific functional and structural traits (Table 4.1).

A seedling consists of a trunk with an apical meristem and an associated leaf compartment. Note that only at this seedling stage, leaf compartments are associated with the trunk. Thereafter leaf compartments are always associated with second order branches. The initial trunk diameter is given by $D_{\text {ini. }}$. Because species differ in their intrinsic height-diameter relationships, the initial height is calculated based on $D_{\text {ini }}$ according to Eq. 4.6. Due to the relationship between leaf area and cross-sectional area of active pipes (Shinozaki et al. 1964), the initial leaf area is coupled to $D_{\text {ini }}$ (Eq. 4.52). Consequently, all seedlings start with a leaf compartment with equal initial leaf area $A_{\mathrm{L}}$, but due to difference in $S L A$, the initial leaf biomass $B_{\mathrm{L}}$ differs among species.

\section{Tree growth}

Simulating tree growth using carbon-based FSTMs involves calculating carbon assimilation and allocation. Whereas the process of carbon assimilation is well-understood, the process of carbon allocation among different tree organs/components is debated (Lacointe 2000; Franklin et al. 2012). Several approaches to simulate carbon allocation have been proposed (Allen, Prusinkiewicz \& DeJong 2005; Franklin et al. 2012; Mäkelä 2012). Here, we largely follow the principles of module autonomy, which state that plants are composed of repetitive modules which respond independently to their local environment (Sprugel et al. 1991; de Kroon et al. 2005). Hence, the assimilated carbon is reinvested locally into production of new leaves and branches (Sprugel et al. 1991). If light is unevenly distributed within canopies, module autonomy will create irregular tree crowns where the leaf biomass is mostly located in favorable, bright regions, which is a pattern often observed in nature.

In this model, the leaf compartments are the independent modules. Leaf compartments represent leaf-pipe elements attached to second order branches. While simulating the development of leaf compartments, all crucial processes (i.e. carbon assimilation, respiration, re-investment of surplus carbon) for both leaves and attached pipes are considered. Consequently, secondary growth of branches and the trunk emerge from the development of all connected leaf compartments. While secondary growth up to most distal branch junctions can sufficiently be simulated applying module autonomy in this model, the costs for primary growth are not explicitly accounted for. To account for theses carbon costs, leaf compartments would have to allocate a certain amount to the apical meristems for primary growth instead of re-investing it locally. This, however, means that leaf compartments cannot act as perfectly autonomous modules. Instead, a set of rules regulating 
carbon allocation among the different potential carbon sinks needs to be defined, which leads back to the initially stated problem concerning carbon allocation modelling methods.

Apart from the uncertainty which method to choose, carbon allocation models are commonly complex and thus computationally costly (Franklin et al. 2012). As model speed is a major constraint in this model, we chose not to use complex methods (e.g. maximization or optimization methods), but rather to simulate the carbon allocation to primary growth using a simple approximation method which largely keeps the autonomy of the leaf compartments. This means that we approximated the costs for primary growth based on the growth during the previous year and distribute these costs among all connected leaf compartments. We regard this approximation method as suitable trade-off between complexity and model speed, but in some situations this might not be appropriate. For instance, primary growth of branches predicted based on previous year growth might be overestimated if a branch collides with the crown of an adjacent tree or if its apical meristem is heavily shaded. In such situation, the apical meristem would commonly send the signal to cease or reduce carbon allocation for primary growth of this branch (King 1991; Stoll \& Schmid 1998; Wilson 2000).

Thus, we integrated control mechanisms regulating primary growth based on the conditions of apical meristems (apical dominance/control). The carbon costs for primary growth according to these described approximation methods are estimated in the first submodel (apical control) in tree growth. For the sake of clarity, this step only approximates the costs that each leaf compartment contributes to primary growth, and not the actual primary growth, which is simulated thereafter. The relative costs for primary growth are usually small compared to the costs for new leaves and secondary growth and thus, approximating these costs seems sufficient. In the second submodel, the carbon balance of all leaf compartments is calculated. This includes carbon assimilation, maintenance, and re-investment into new leaf and woody biomass. The change in leaf area/biomass and the leaf area/biomass production results from these processes. Secondary growth resulting from the carbon balance of all connected leaf compartments, as well as primary growth of all branches and the trunk is simulated in the submodel structural growth.

\section{Apical control}

Each leaf compartment forms a leaf-pipe element whose pipe length $L_{\mathrm{PS}}$ is calculated based on the relative position of the leaf compartment in the tree.

$$
L_{P S}=P_{L C}{ }^{Z}+\sqrt{\left(P_{L C}{ }^{X}-P_{T}{ }^{X}\right)^{2}+\left(P_{L C}{ }^{Y}-P_{T}{ }^{Y}\right)^{2}}
$$




\section{Functional-structural forest model}

where $P_{\mathrm{LC}}{ }^{\mathrm{X}}, P_{\mathrm{LC}}^{\mathrm{Y}}$ and $P_{\mathrm{LC}}^{\mathrm{Z}}$ are the spatial coordinates (centroids) of the leaf compartment, and $P_{\mathrm{T}}{ }^{\mathrm{X}}$ and $P_{\mathrm{T}}{ }^{\mathrm{Y}}$ are the coordinates of the trunk (we selected this simple method to approximate $L_{\mathrm{PS}}$ for reasons of efficiency; calculating $L_{\mathrm{PS}}$ based on the tree topology requires graph queries in GroIMP, which are computationally demanding). By controlling the maintenance and construction cost of pipes, $L_{\mathrm{PS}}$ influences the carbon balance of a leaf compartment. Now, we assume that not the entire carbon assimilated by a leaf compartment is locally re-invested, but that a certain proportion is allocated to the apical meristem of the connected first order branch and the trunk for primary growth. These additional costs for primary growth are taken into account by increasing the pipe length according to the predicted, potential length growth of the trunk and the first order branch. To predict the potential length growth in the current time step, we assume that the diameter increase equals the diameter increase in the previous year. On the basis of allometric relationships, the potential length increase can then be predicted:

$$
\begin{aligned}
& L_{T}=100 \cdot L D_{T} \cdot D_{T}^{2 / 3} \\
& L_{B}=100 \cdot L D_{B} \cdot S_{F}^{o_{B}} \cdot D_{B}^{2 / 3}
\end{aligned}
$$

where $L_{\mathrm{T}}, L_{\mathrm{B}}$ is the length and $D_{\mathrm{T}}, D_{\mathrm{B}}$ is the diameter of trunks and branches, respectively. $L D_{\mathrm{B}}$ and $L D_{\mathrm{T}}$ are species-specific allometric shape parameter, with higher values representing more slender trunks or branches, and $S_{\mathrm{F}}$ is a species-specific factor regulating the shortening of branches with their order $O_{\mathrm{B}}\left(S_{\mathrm{F}}<1\right)$. The factor 100 converts $\mathrm{m}$ to $\mathrm{cm}$ as in such allometric relationships the diameter is generally given in $\mathrm{cm}$ and the length in $\mathrm{m}$. These allometric relationships are based on McMahon (1971), who described that the critical length $L_{\mathrm{Cr}}$ for buckling is proportional to the diameter $D$ raised to the $2 / 3$ power.

$$
L_{C r}=100 \cdot L D_{C r} \cdot D^{2 / 3}=100 \cdot 4.39 \cdot D^{2 / 3}
$$

where $L D_{\mathrm{Cr}}$ is the critical allometric shape parameter. This parameter is influenced by the ratio between wood density and elastic modulus, which is fairly constant in green wood, with the estimated values of $L D_{\mathrm{Cr}}=4.39$ being regarded as upper limit across many tree species (McMahon 1971). Trees species commonly include stability safety factors, meaning that they grow below the critical length and hence $L D_{\mathrm{B}}$ and $L D_{\mathrm{T}}<L D_{\mathrm{Cr}}$.

Based on Eqs. 4.6 and 4.7, the potential length increase can be predicted, but assuming that trunks and branches always grow according to allometric relationships might be too simplistic. Controlled by hormones, the allocation of carbon to apical meristems can either be inhibited (apical control; Wilson 2000) or intensified (apical dominance; Cline 1997), which modifies the 
shape of branches/trunks substantially. For instance, trees often intensify carbon allocation to the trunk apical meristem when they are shaded, most likely to quickly reach higher zones with more light (Poorter 1999). This process leads to more slender trunks. In contrast, branches commonly inhibit primary growth when their apical meristem is shaded or when branches collide. To account for these processes, we integrated additional mechanisms controlling the potential length increase.

For trunks, we assume that intensified carbon allocation to the trunk apical meristem is initiated when the light intensity at the apical meristem $I_{\mathrm{M}}$ is below a species-specific threshold $I_{\mathrm{T}}$. The relative intensification in height growth $L_{\text {inc }}$ (compared to the regular allometric growth) is implemented as function of the light intensity.

$$
L_{i n c}=\beta_{D} \cdot e^{-\left(\frac{I_{M}}{I_{T}}\right)^{\beta_{S}}}
$$

where $\beta_{\mathrm{S}}$ defines the shape of the function and $\beta_{\mathrm{D}}$ is the maximum relative deviation. When considering $L_{\text {inc }}$, the potential length increase of the trunk $\Delta L_{\text {TPotRg }}$ is calculated as

$$
\Delta L_{T P o t R g}=L D_{T} \cdot\left(\left(2 \cdot D_{T(y 0)}-D_{T(y-1)}\right)^{2 / 3}-\left(D_{T(y 0)}\right)^{2 / 3}\right) \cdot\left(1+L_{i n c}\right)
$$

where $D_{\mathrm{T}(\mathrm{y} 0)}$ and $D_{\mathrm{T}(\mathrm{y}-1)}$ are the diameter at the beginning of the current time step and at the beginning of the previous time step, respectively. Continued apical dominance might lead to slender trunks which could potentially exceed the critical length (Eq. 4.8). Thus, the potential length increase up to the critical length $\Delta L_{\mathrm{TPotCr}}$ is additionally estimated.

$$
\Delta L_{T P o t C r}=L D_{C r} \cdot\left(2 \cdot D_{T(y 0)}-D_{T(y-1)}\right)^{2 / 3}-L_{T(y 0)}
$$

where $L_{\mathrm{T}(\mathrm{y} 0)}$ is the length of the trunk at the beginning of the current time step. Each species has a maximum height $L_{\mathrm{TMax}}$ resulting from its functional traits (Eqs. 4.42 and 4.43 ), which additionally limits the potential length increase. The potential length increase up to the maximum height $\Delta L_{\text {TPotMax }}$ is calculated as

$$
\Delta L_{T P o t M a x}=L_{T M a x}-L_{T(t)}
$$




\section{Functional-structural forest model}

Consequently, the effective potential length increase of the trunk $\Delta L_{\text {TPot }}$ is the minimum of these three variables.

$$
\Delta L_{T P o t}=\min \left(\Delta L_{T P o t R g}, \Delta L_{T P o t C r i t}, \Delta L_{T P o t M a x}\right)
$$

For first order branches, we integrated two mechanisms regulating their potential length increase. First, branches stop to grow in length if the light intensity at the apical meristem is not sufficient to allow positive growth, i.e. if the photosynthetic rate $G R_{\text {Pot }}<0$ (see next section). Second, branches stop to grow in length if adjacent trees grow in the immediate surroundings, i.e. it is tested if there are any tree components from other trees in the same voxel as the apical meristem (this mechanism can be disabled by setting the global parameter $B r$ Collide $=0$ ). In both cases, the potential length increase of branches is set to $\Delta L_{\mathrm{BPot}}=0$. This means that branches may stop growing in length while continuing to grow in diameter, and thus they might deviate from their regular allometric relationship (Eq. 4.7). If, after a period of apical control, primary growth would be reactivated, for instance by more favorable light conditions, length increase would not be appropriately simulated based on Eq. 4.7, as branches could show an unrealistically huge increase in length in one time step. Thus, the potential length increase of branches $\Delta L_{\mathrm{BPot}}$, when not limited by low light or adjacent trees, is calculated as

$$
\Delta L_{B P o t}=L D_{B} \cdot S_{F}^{o_{B}} \cdot\left(\left(2 \cdot D_{B(y 0)}-D_{B(y-1)}\right)^{2 / 3}-\left(D_{B(y 0)}\right)^{2 / 3}\right)
$$

This assumes that the increase in length at a given diameter can be approximated by the length increase if the branch would strictly have grown according to its regular allometric growth routine.

After the potential length increase of the first order branch and trunk associated with a leaf compartment has been calculated, the effective pipe length $L_{\mathrm{P}}$ of each leaf compartment is updated accordingly

$$
L_{P}=L_{P S}+\Delta L_{T P o t}+\Delta L_{B P o t}
$$

The effective pipe length $L_{\mathrm{P}}$ thus includes the pipe length of the leaf compartment according to its position within the tree plus the potential length increase of its associated first order branch $\Delta L_{\mathrm{BPot}}$ and trunk $\Delta L_{\mathrm{TPot}}$. 


\section{Carbon balance}

This submodel simulates the carbon balance of all leaf compartments, which includes carbon assimilation and respiration, as well as loss of and investment into new biomass. When carbon assimilation exceeds the respiration/maintenance cost for leaves and connected pipes, the surplus carbon is invested into new leaf and pipe biomass. The sum of all leaf compartments of a tree comprises its total leaf and sapwood biomass.

To understand this submodel, we distinguish differences between voxels and leaf compartments. Voxels are not associated with any tree parts and contain aggregated information like the average light intensity (Eq. 4.4) or the total leaf area of all leaf compartments in the voxel (Eq. 4.1). If a new second order branch is generated within a voxel, a new leaf compartment is generated, which means that within the same voxel multiple leaf compartments may exit. Likewise, if a second order branch grows into a new voxel, a new leaf compartment is generated, which means that a leaf compartment is always associated with a specific part of a second order branch and consequently, each branch may have multiple leaf compartments.

While the model proceeds in annual time steps, many processes take place at shorter time intervals. For instance, new leaves may be produced, which by increasing the photosynthetically active area positively influence the annual carbon balance. To better account for these effects, our model considers daily rates and simulates the development of the leaf compartments during one year. The annual rates are then estimated as the result of these simulations after $t_{\text {year }}=360$ days. An additional advantage of this approach is that seasonal forests can be simulated by reducing $t_{\text {year }}$.

Each leaf compartment contains leaves whose leaf dry mass $B_{\mathrm{L}}$ and leaf area $A_{\mathrm{L}}$ can be mutually converted via the species-specific SLA.

$$
A_{L}=B_{L} \cdot S L A
$$

Leaves are the photosynthetically active organs and the gross carbon assimilation rate per unit of leaf dry mass $C_{\text {gross }}$ is calculated as hyperbolic Michaelis-Menten function.

$$
C_{\text {gross }}=\frac{G_{\max } \cdot I}{k+I} \cdot S I
$$

where $I$ is the light intensity at the leaf compartment (superscripts depicting spatial coordinates are not explicitly given here), and $G_{\max }$ and $k$ are species-specific traits (Table 4.1). The site index $S I[0,1]$ describes the relative environmental quality of the site and can be understood as 


\section{Functional-structural forest model}

aggregated information on all extrinsic factors which are not explicitly simulated in our model, e.g. nutrient, water availability or temperature. A SI of 1 thus refers to optimum external factors and no resource limitation.

Maintenance costs have to be paid for both the leaves $\left(R_{\mathrm{L}}\right)$ and the sapwood, i.e. the pipes $\left(R_{\mathrm{WTot}}\right)$. While $R_{\mathrm{L}}$ is a species-specific trait, the maintenance rate for connected pipes per unit of leaf dry mass $R_{\mathrm{WTot}}$ depend on the position of the leaf compartment within the tree and are calculated as follows.

$$
R_{w T o t}=R_{w} \cdot \frac{L_{P}}{L P_{\text {ratio }}} \cdot \rho_{W} \cdot S L A
$$

where $R_{\mathrm{w}}$ are the general respiration costs per dry mass of pipes. Because we assume a fixed ratio between leaf area and cross-sectional area of connected pipes $\left(L P_{\text {ratio }}\right)$, the total dry mass of pipes per unit of leaf dry mass can be calculated based on the length of the pipe system $L_{\mathrm{P}}$, the wood density $\rho_{\mathrm{w}}$ and the specific leaf area $S L A$.

Subtraction of the maintenance rates from the gross carbon assimilation rate yields the net carbon assimilation rate per unit of leaf mass $C_{\text {net. }}$.

$$
C_{n e t}=C_{\text {gross }}-R_{L}-R_{W T o t}
$$

If $C_{\text {net }}$ is positive, the surplus carbon can be reinvested into new leaf biomass and associated pipes. The amount of leaf dry mass that can be produced per unit of assimilated carbon $C_{B}$ depends on the ratio of leaf dry mass to pipe dry mass and can be calculated as follows.

$$
C_{B}=\left(\left(C B L_{\text {ratio }}+\frac{L_{P}}{L P_{\text {ratio }}} \cdot C B W_{\text {ratio }} \cdot \rho_{W} \cdot S L A \cdot P_{R U}\right) \cdot C_{O}\right)^{-1}
$$

While $C_{\text {net }}$ is expressed in g carbon, the leaf and woody biomass is expressed in dry mass. Thus, the C-mass to biomass ratio of wood $C B W_{\text {ratio }}$ and of leaves $C B L_{\text {ratio }}$ is considered here. In addition, we assume that a certain proportion of $\mathrm{C}$ invested into new leaf or woody biomass is lost as growth respiration $C_{\mathrm{O}} . P_{\mathrm{RU}}[0,1]$ is the pipe-reuse factor which specifies the ratio of new pipes to reused old pipes when new leaves are generated. When strictly following the pipe-model theory, new pipes are generated for each new leaf, while old pipes are converted from sapwood to heartwood when the leaves die (i.e. $P_{\mathrm{RU}}=1$ ). However, it is assumed that a certain proportion of old pipes can be reused (it is difficult to observe/measure this behavior, but see Mäkelä, 1986, 
2002). We thus added the possibility to include this mechanism $\left(P_{\mathrm{RU}}<1\right)$. For the sake of clarity, $C_{\mathrm{B}}$ defines how much of the assimilated carbon is invested into leaf biomass considering the carbon costs for the pipes associated with the leaves. This means that, when calculating the total annual leaf biomass production based on $C_{\text {net }}$ and $C_{\mathrm{B}}$, both the maintenance costs and the construction costs for leaves and pipes are fully included. From this it also follows that, due to the fixed leaf area to pipe area ratio $\left(L P_{\text {ratio }}\right)$, secondary growth is directly linked to the total annual leaf biomass production (next section).

Multiplication of $C_{\text {net }}$ and $C_{\mathrm{B}}$ yields the relative growth rate of leaf biomass. Without considering leaf losses, the change in leaf biomass $B_{\mathrm{L}}$ over time in a leaf compartment could thus be described by the following ordinary differential equation.

$$
\frac{d B_{L}}{d t}=C_{B} \cdot C_{n e t} \cdot B_{L}
$$

It can be seen that, if $C_{\text {net }}$ is negative, leaf biomass is lost. In addition, as the average leaf lifespan $L L$ is an additional species-specific trait, leaves are constantly lost at a rate of $1 / L L$. Thus, when considering both the (potential) production term $\left(C_{\text {net }} C_{\mathrm{B}}\right)$ and the loss term $(1 / \mathrm{LL})$, the change in leaf biomass $B_{\mathrm{L}}$ over time is

$$
\frac{d B_{L}}{d t}=C_{B} \cdot C_{n e t} \cdot B_{L}-\frac{1}{L L} \cdot B_{L}=\left(C_{B} \cdot C_{n e t}-\frac{1}{L L}\right) \cdot B_{L}=G R_{p o t} \cdot B_{L}
$$

Positive growth of leaf biomass is possible only if the carbon production rate is higher than the carbon loss rate (i.e. $G R_{\text {pot }}>0$ ). Solving this equation yields the leaf biomass as a general function of time.

$$
B_{L}(t)=B_{L(t 0)} \cdot e^{G R_{p o t} \cdot t}
$$

where $B_{\mathrm{L}(0)}$ is the initial leaf biomass. Eq. 4.23 describes the temporal dynamics of leaf biomass by an exponential function to the base $e$, implying that surplus carbon is directly reinvested into new leaf biomass, which immediately participates in photosynthesis. However, in reality, surplus carbon is first allocated to leaf primordia, which develop into photosynthetically active organs with a time lag (Hallé et al. 1978). To account for this, we use the base 2 instead of $e$ in our simulations.

$$
B_{L}(t)=B_{L(t 0)} \cdot 2^{G R_{p o t} \cdot t}
$$




\section{Functional-structural forest model}

As daily rates are used (Eqs. 4.17-4.19), the leaf biomass at the end of one year $B_{\mathrm{L}(\mathrm{y}+1)}$ can be calculated by inserting the number of suitable days $t_{\text {year }}$ and the initial biomass at the beginning of the year $B_{\mathrm{L}(\mathrm{y} 0)}$.

$$
B_{L(y+1)}=B_{L(y 0)} \cdot 2^{G R_{\text {pot }} \cdot t_{\text {year }}}
$$

Eq. 4.26 constitutes the basic rule to simulate the leaf biomass dynamics. Under sustained favorable light conditions this equation would predict a potentially infinite accumulation of leaf biomass, which is not adequate as leaf compartments are limited by their discrete volumes $\left(1 \mathrm{~m}^{3}\right)$. To get a more realistic behavior of the leaf biomass dynamics, two modifications are implemented. First, a global upper maximum of the total leaf area per voxel $\left(A_{\text {LMax }}\right)$ is applied. Plants tend to avoid self-shading through efficient arrangements of leaf areas (King et al. 1997), and thus a maximal leaf area instead of a maximal leaf biomass is defined. Second, a speciesspecific maximum leaf production per leaf compartment $\left(A_{\text {LProdMax }}\right)$ is implemented. The production of new leaves and branch segments is regulated by the activity of meristems, which generally follow specific intrinsic architectural rules (Hallé et al. 1978). Existing parts of branches do not have the potential to produce an unlimited number of new meristems capable of differentiating into leaves. $A_{\text {LProdMax }}$ can thus be understood as the maximum amount of leaves, expressed as leaf area that can be produced within a leaf compartments associated with a specific section of a second order branch. As long as the total amount of leaves produced is lower than $A_{\text {LProdMax }}$, new leaf biomass can be produced if the light conditions are suitable. In the following, the modifications of the basic Eq. 4.25 under consideration of $A_{\text {LMax }}$ and $A_{\text {LProdMax }}$ are described. At first, to prevent the total leaf area in a voxel $A_{\text {LTot }}$ (Eq. 4.1) to exceed the maximum $A_{\mathrm{LMax}}$, the theoretical maximal growth rate $G R_{\max }$ is calculated so that $A_{\mathrm{LTot}}=A_{\mathrm{LMax}}$ when $G R_{\max }$ is applied in Eq. 4.25 instead of $G R_{\text {pot }}$.

$$
G R_{\text {max }}=\frac{\log _{2}\left(\frac{A_{L M a x}}{A_{L T o t}}\right)}{t_{\text {year }}} \quad\left(A_{\text {LTot }}, t_{\text {year }} \neq 0\right)
$$

Note that the necessary conditions are always satisfied because naturally $t_{\text {year }}>0$ and, as leaf compartments without any leaf biomass are removed, for all existing leaf compartment $A_{\mathrm{L}}>0$ and thus $A_{\text {LTot }}>0$. The effective growth rate $G R$ is then calculated as follows.

$$
G R=\min \left(G R_{\text {max }}, G R_{\text {pot }}\right)
$$


Integrating the effective growth rate $G R$ in Eq. 4.25 ensures that the total leaf area of all leaf compartments in a voxel never exceeds $A_{\text {LMax }}$. To ensure that the production maximum $A_{\text {LProdMax }}$ is never exceeded, it is essential to log the total leaf area production of a leaf compartment $A_{\text {LProdTot }}$.

$$
A_{\text {LProdTot }(y+1)}=A_{\text {LProdTot }(y 0)}+A_{\text {LProd }}
$$

where $A_{\mathrm{LProd}}$ is the annual leaf area production. Based on $A_{\mathrm{LProdTot}}$ and $A_{\mathrm{LProdMax}}$, the theoretical maximal leaf area production in the current time step $A_{\text {LProdTheo }}$ can be estimated.

$$
A_{\text {LProdTheo }}=A_{\text {LProdMax }}-A_{\text {LProdTot }}
$$

Dividing $A_{\text {LProdTheo }}$ by the SLA yields the theoretical maximal leaf biomass production in the current time step $B_{\text {LProdTheo. }}$.

$$
B_{\text {LProdTheo }}=\frac{A_{\text {LProdTheo }}}{S L A}
$$

Consequently, it has to be verified whether the annual leaf biomass production, when applying the effective growth rate $G R$ (Eq. 4.28), would exceed this maximum. Please note that the annual leaf biomass production is not the same as the annual change in leaf biomass (Eq. 4.25), which is the result of leaf biomass production minus leaf loss. Thus, these two processes have to be separated. Using the effective growth rate $G R$, the potential leaf biomass production $B_{\text {LProdPot }}$ can be calculated as follows.

$$
B_{\text {LProdPot }}=\left\{\begin{array}{cc}
\left(\frac{B_{L(y 0)}}{G R} e^{G R \cdot t_{\text {year }}}-\frac{B_{L(y 0)}}{G R}\right) \cdot\left(G R+\frac{1}{L L}\right) & \text { if } G R \neq 0 \\
B_{L(y 0)} \cdot \frac{t_{\text {year }}}{L L} & \text { if } G R=0
\end{array}\right.
$$

where $B_{\mathrm{L}(\mathrm{y} 0)}$ initial leaf biomass of the leaf compartment. The case discrimination is necessary because the regular Eq. to calculate $B_{\mathrm{LProdPot}}$ is not defined if $G R=0$ (the additional necessary condition LL $\neq 0$ is always satisfied, as the leaf lifespan naturally is larger than zero). If $G R=0$, the leaf production rate must equal the leaf loss rate and consequently $B_{\mathrm{LProdPot}}$ can be calculated based on the leaf loss rate $1 / \mathrm{LL}$, which is constant and species-specific rate. The effective leaf biomass production $B_{\mathrm{LProd}}$ is simply calculated by applying the minimum function on $B_{\mathrm{LProdTheo}}$ and $B_{\text {LProdPot. }}$ 


$$
B_{\text {LProd }}=\min \left(B_{\text {LProdPot }}, B_{\text {LProdTheo }}\right)
$$

Recapitulating, the effective leaf biomass production $B_{\mathrm{LProd}}$ is the total amount of leaf biomass produced by a leaf compartment under consideration of $A_{\text {LMax }}$ and $A_{\text {LProdmax }}$. Now that the leaf biomass production for each leaf compartment is known, the change in leaf biomass equivalent to Eq. 4.25 has to be simulated. In Eq. 4.25 we assume that both the production rate and the loss rate are constant throughout the entire year. If the leaf production maximum $A_{\text {LProdMax }}$ would not be reached during a time step, which is the case if $B_{\text {LProdTheo }}>=B_{\text {LProdPot }}$, application of Eq. 4.25 would properly estimate the change in leaf biomass. However, if $A_{\text {LProdMax }}$ would be reached, i.e. if $B_{\text {LProdTheo }}<B_{\text {LProdPot }}$, Eq. 4.25 could not be applied. In this case, the production of new leaf biomass would stop during the year as soon as $A_{\text {LProdMax }}$ is reached. To account for this, we divide the year into two periods. In the first period, both leaf production and leaf loss are active and thus the leaf biomass dynamics can follow its regular mechanisms. In the second period, as soon as $A_{\text {LProdMax }}$ is reached, leaf production ceases and only leaf loss remains active. Based on the known leaf biomass production $B_{\mathrm{LProd}}$ the length of the first period $t_{\mathrm{p}}$, i.e. the 'productive time period', can be calculated as follows.

$$
t_{p}=\left\{\begin{array}{cl}
\frac{\ln \left(\frac{B_{L P r o d} \cdot G R}{B_{L(y 0)} \cdot\left(G R+\frac{1}{L L}\right)}+1\right)}{\frac{G R}{B_{L P r o d} \cdot L L}} & \text { if } G R \neq 0 \\
B_{L(y 0)} & \text { if } G R=0
\end{array}\right.
$$

Under consideration of $t \mathrm{p}$, the annual change in leaf biomass can be calculated as follows.

$$
B_{L(y+1)}=B_{L(y 0)} \cdot 2^{G R \cdot t_{p}} \cdot 2^{-\frac{\left(t_{y e a r}-t_{p}\right)}{L L}}
$$

This equation thus replaces the basic Eq. 4.25 and constitutes the final equation based on which the annual change in leaf biomass for each leaf compartment is calculated. This equation covers all possible scenarios. First, if there is no limitation in annual biomass production imposed by $A_{\text {LProdMax }}$, the productive time period becomes $t_{\mathrm{P}}=t_{\text {year }}$ and thus Eq. 4.34 equals Eq. 4.25. Second, if $A_{\mathrm{LProdMax}}$ is already reached at the beginning of the time step, i.e. if $B_{\mathrm{LProd}}=0$, the productive time period becomes $t_{\mathrm{P}}=0$. In this case, only leaf loss is considered in Eq. 4.34. Third, if $A_{\text {LProdMax }}$ is reached during the annual time step, the productive time period is estimated by Eq. 4.34 so that it exactly describes the time needed to reach $A_{\text {LProdMax }}$. 


\section{Structural growth}

This submodel simulates the structural growth of trees and includes changes in the state variables of existing tree components, establishment of new tree components and removal of old ones. All of these processes result from the carbon balance. The secondary growth results from the leaf biomass production in all topologically connected leaf compartments. The primary growth, in turn, is related to secondary growth via allometric relationships. Secondary and primary growth involve both changes in the state variables of existing tree components and the establishment of new ones. As we assume that photosynthetically inactive, leafless branches are shed, the removal of tree components is also a direct outcome of the carbon balance. In the following, after an introduction to the modelling software GroIMP used here, we describe how the results of the preceding submodel are translated into structural growth. The structural traits are described in detail at the end of this section.

This model is implemented using the open-source software GroIMP (Growth Grammar Interactive Modelling Platform; available under the GNU General Public License at www.grogra.de). GroIMP is a 3D modelling platform suited to simulate the structural growth of plants. Here we illustrate the main concepts essential for understanding the functioning of this submodel (refer to Kniemeyer (2008) for detailed information on GroIMP). In GroIMP, relational growth grammars are implemented by the programming language XL, which is a graph-based extension of the Lindenmeyer-Systems (L-Systems), a formal language for the description of plant structure (Lindenmayer 1968a; b). XL is built on top of the programming language Java and thus both the XL-specific set of rules tailored to model plant structures, as well as the general Java classes can be used. Graphs are the underlying data structure in XL defining the tree topology. They describe how the different tree components of a tree, which can be defined as 3D geometric objects, are interconnected and spatially arranged to one another.

In our model, the trunk is defined as $3 \mathrm{D}$ cone, while the branch segments are defined as $3 \mathrm{D}$ cylinders. Taking into account the state variables of the tree components, the graph of each tree can thus be interpreted as 3D tree structure (Fig. 4.1a). XL contains a set of rules to modify the graph and thereby to induce structural growth. A rule consists of a graph query, an expressions used to select specific parts of the graph, and a statement which specifies how to modify the selected parts. For example, a query selects all second order branches not connected to any leaf compartments and a statement removes them. As another example, the rule to sum up the leaf biomass production over all leaf compartments topologically connected to a branch segment, and to change state variables of the branch segment accordingly, would, for each individual branch segment, traverse through the graph. Replacement rules are also common types of rules. Such rules select specific parts of a graph and replace them with other graph nodes, which, in this model, are the tree components. Meristems are the place of growth in trees and accordingly, apical 


\section{Functional-structural forest model}

meristems are replaced by other tree components to simulate primary growth in this model. Using these rules, the results of the carbon balance submodel are translated into structural growth in XL.

The first step in this submodel is to calculate the updated total diameter of branches $D_{\mathrm{B}(\mathrm{y}+1)}$ and the trunk $D_{\mathrm{T}(\mathrm{y}+1)}$ based on the sum of the annual leaf biomass production $B_{\mathrm{LProd}}$ of all topologically connected leaf compartments (Eq. 4.33). As the maintenance and construction costs of the pipes associated with leaf compartments have already been considered, the updated diameter is estimated using the ratio $L P$ ratio.

$$
\begin{aligned}
& D_{T(y+1)}=2 \cdot \sqrt{\frac{\left.\left(\frac{D_{T(y 0)}}{2}\right)^{2} \cdot \pi+\frac{\sum B_{L P r o d}}{L P_{\text {ratio }}} \cdot S L A \cdot P_{R U}\right)}{\pi}} \\
& D_{B(y+1)}=2 \cdot \sqrt{\frac{\left(\left(\frac{D_{B(y 0)}}{2}\right)^{2} \cdot \pi+\frac{\sum B_{L P r o d}}{L P_{\text {ratio }}} \cdot S L A \cdot P_{R U}\right)}{\pi}}
\end{aligned}
$$

Based on the updated diameter, the updated length of the trunks and branches can be calculated via allometric relationships (Eqs. 4.6 and 4.7) under consideration of the mechanisms of apical control/dominance. For branches, the potential length increase was set to $\Delta L_{\mathrm{BPot}}=0$ either if their apical meristems are heavily shaded or if they collide with other trees (see submodel apical control). On this basis, the updated branch length $L_{\mathrm{B}(\mathrm{y}+1)}$ is calculated as follows.

$$
L_{B(y+1)}=\left\{\begin{array}{cr}
L_{B(y 0)}+L D_{B} \cdot S_{F} o_{B} \cdot\left(\left(D_{B(y+1)}\right)^{2 / 3}-\left(D_{B(y 0)}\right)^{2 / 3}\right) & \Delta L_{B P o t} \neq 0 \\
L_{B(y 0)} & \Delta L_{B P o t}=0
\end{array}\right.
$$

For trunks, no apical control mechanisms preventing length growth under unfavorable conditions are integrated. Rather, length growth can be intensified, and the relative intensification in height growth is expressed by $L_{\text {inc }}$ (Eq. 4.9; see submodel apical control for details). The regular and otherwise unrestricted updated length of a trunk $L_{\mathrm{TRg}}$ can thus be estimated according to Eq. 4.10 as follows.

$$
L_{T R g}=L_{T(y 0)}+L D_{T} \cdot\left(\left(D_{T(y+1)}\right)^{2 / 3}-\left(D_{T(y 0)}\right)^{2 / 3}\right) \cdot\left(1+L_{i n c}\right)
$$


While the environmental conditions at the trunk apical meristem do not limit height growth, it can be limited by the critical height $L_{\text {Trr }}$ (Eq. 4.8) or the maximum trunk height $L_{\mathrm{TMax}}$. Thus, the effective updated height of the tree $L_{\mathrm{T}(\mathrm{y}+1)}$ is calculated as follows.

$$
L_{T(y+1)}=\min \left(L_{T R g}, L_{T C r}, L_{T M a x}\right)
$$

The critical length $L_{\mathrm{Tcr}}$ is estimated based on $D_{\mathrm{T}(\mathrm{y}+1)}$ (Eq. 4.8). The maximum trunk height $L_{\mathrm{TMax}}$ is a species-specific variable emerging from the functional traits, which is described in the following. A positive carbon balance in a leaf compartment can only be maintained if the carbon gain exceeds the carbon cost, i.e. if $G R_{\text {Pot }}>0$ (Eq. 4.22). The carbon gain generally increases with increasing light intensity $I$ (Eq. 4.17), while the carbon costs increase with the pipe length $L_{\mathrm{P}}$ (Eqs. 4.18 and 4.20). At the theoretical maximal light intensity $I_{\max }$, there is thus a maximum pipe length $L_{\mathrm{PMax}}$ at which the carbon gain and the carbon costs are equal, i.e. at which $G R_{\mathrm{Pot}}=0$.

$$
G R_{P o t}=C_{B} \cdot C_{n e t}-\frac{1}{L L}=0
$$

By substitution of Eqs. 4.17-4.20 into Eq. 4.40, and setting $I=I_{\max }$ and $L_{\mathrm{P}}=L_{\mathrm{PMax}}$, the maximum pipe length $L_{\mathrm{PMax}}$ can be calculated.

$$
L_{P M a x}=\frac{\frac{G_{\max } \cdot I_{\max } \cdot S I}{k+I_{\max }}-R_{L}-\frac{C B L_{\text {ratio }} \cdot C_{O}}{L L}}{\frac{R_{w} \cdot \rho_{W} \cdot S L A}{L P_{\text {ratio }}}+\frac{C_{O} \cdot C B W_{\text {ratio }} \cdot \rho_{W} \cdot S L A \cdot P_{R U}}{L P_{\text {ratio }} \cdot L L}}
$$

This equation contains only global constants and species-specific leaf and wood traits, making $L_{\mathrm{PMax}}$ an emergent species-specific variable. $L_{\mathrm{PMax}}$ thus represents the maximum pipe length under the given plot quality (i.e. site index $S I$ ), and the absolute maximum $L_{\mathrm{PMaxAbs}}$ can be estimated by setting $S I=1$.

As each tree is assumed to have only one trunk, the trunk length should never exceed $L_{\text {PMax }}$. For branches, testing if the length of the pipe system exceeds $L_{\mathrm{PMax}}$ is not necessary, as this is implicitly done in the apical control submodel: if the carbon balance at the apical meristem would be negative $\left(G R_{\mathrm{Pot}}<0\right)$, which is always the case if $L_{\mathrm{P}}>L_{\mathrm{PMax}}$, primary branch growth ceases (Eq. 4.38). However, if the maximum trunk height is defined as $L_{\mathrm{PMax}}$ and the apical control for branches is applied, the shape of trees can appear unrealistic. This is because $L_{\text {PMax }}$ describes the theoretical, maximum pipe length at maximum light intensity, while the apical control of the branches considers the actual light intensity in the voxels. Hence, particularly when a trunk is 


\section{Functional-structural forest model}

close to $L_{\mathrm{PMax}}$, it might be that it continues to grow in length, while new lateral branches might not. In such a tree, it would appear as if the main trunk would grow through its own tree crown. To prevent this behavior, we introduce a safety factor for trunk growth $S T(S T<1)$ that defines the ratio of the actual maximum trunk height to the theoretical maximum pipe length $L_{\mathrm{PMax}}$. The maximum trunk height is thus given as

$$
L_{T M a x}=S T \cdot L_{P \operatorname{Max}}
$$

While a trunk stops to grow in height at $L_{\mathrm{TMax}}$ (Eq. 4.40), lateral branches may grow above this point, by this creating realistic looking tree crowns (note that $S T$ is defined as a global constant and thus $L_{\mathrm{TMax}}$ remains a species-specific emergent trait).

While trunks are simply updated based on the updated state variables, updating the visual representation of branches is more complicated because branches are described at two scales. At the coarse scale, a branch is described by its total length and diameter, what has been calculated above. At the fine scale, a branch is described by a set of topologically connected segments, which may further be connected to higher order segments. These branch segments at the fine scale are the tree components which are visually represented in GroIMP and thus, the state variables of the existing branch segments have to be updated and new branch segments have to be introduced according to the simulated total length and diameter growth. This means that in this model the total length growth of a branch is calculated first, resulting in establishment of a corresponding number of branch segments and not vice versa as in most FSTMs. We choose this two-scale approach as trade-off between computational costs and visual aspects. Treating the branch as an entity at the coarse scale reduced the computational cost by reducing the number of graph queries. If these coarse-scale branches would be visually displayed, the tree structure would appear unrealistic and consequently, we used smaller branch segments for visualization. This lends more realistic, irregular branch structures, including twisting of branches or effects of photo- or gravitropism (Fig. 4.6a). In the following, the essential information to calculate the fine-scale branch segments is provided.

Second order branches are the simplest case because they cannot ramify into higher order branches. The apical meristem of each second order branch is thus replaced by a segment with a length $L_{\mathrm{S}}$ corresponding to the total length increase.

$$
L_{S}=L_{B(y+1)}-L_{B(y 0)}
$$


The diameter of this new and all existing second order branch segments $D_{\mathrm{S}}$ are updated based on their distance to their branch base $D I_{\mathrm{S}}$ (Fig. 4.6a).

$$
D_{S}=D_{B(y+1)} \frac{L_{B(y+1)}-D I_{S}}{L_{B(y+1)}}
$$

The situation is more complex for first order branches because their primary growth might induce the establishment of new lateral branches. Thus, the number of lateral branches, as well as the length/diameter of both internodes and lateral branches, needs to be estimated (Fig. 4.6b). At first, the internode length $L_{\mathrm{IB}}$ which defines the distance between two branching points is calculated. In reality, the average internode length usually varies between species, but often also within individuals. A positive correlation between the total annual length growth and the internode length has been observed within individual trees (King 1997; Nicolini et al. 2003). On this basis, a flexible internode length $L_{\mathrm{IB}}$ as function of total annual length growth $\left(\Delta L_{\mathrm{B}}=L_{\mathrm{B}(\mathrm{y}+1)}-L_{\mathrm{B}(\mathrm{y} 0)}\right)$, which can vary between the species-specific minimum $L_{\mathrm{IBMin}}$ and maximum internode length $L_{\mathrm{IBMax}}$, is used here.

$$
L_{I B}=L_{I B M i n}+\frac{\left(L_{I B M a x}-L_{I B M i n}\right)}{1+e^{\left(-k_{\operatorname{Int}} \cdot\left(\left(L_{B(y+1)}-L_{B(y 0)}+L_{\text {SLast }}\right)-L_{I B M a x} \cdot 2\right)\right)}}
$$

where $L_{\text {SLast }}$ is the lengths of the last apical branch segment (Fig. 4.6b) and $k_{\text {Int }}$ is a global constant controlling the change of $L_{\mathrm{IB}}$ with total length growth. For clarity, $L_{\mathrm{IB}}$ can differ between different branches of an individual tree, and obviously also from year to year, but for an individual branch we assume $L_{\mathrm{IB}}$ to be invariable within one year. Based on $L_{\mathrm{IB}}$, the number of new lateral branches of a single branch $n_{\text {BLat }}$ can thus be calculated.

$$
n_{B L a t}=f \operatorname{floor}\left(\frac{\left(L_{B(y+1)}-L_{B(y 0)}+L_{\text {SLast }}\right)}{L_{I B}}\right)
$$

Naturally, the number of new branch segments is $n_{\mathrm{BSeg}}=n_{\mathrm{BLat}}+1$. Since the total length growth $\Delta L_{\mathrm{B}}$ is usually not an integer multiple of the internode length $L_{\mathrm{IB}}$, the first and the last segment may be smaller than $L_{\mathrm{IB}}$ (Fig. 4.6b). The length of the first segment $L_{\text {SFirst }}$ is estimated as follows.

$$
L_{S F i r s t}=L_{I B}-L_{S L a s t}
$$




\section{Functional-structural forest model}

where $L_{\text {SLast }}$ refers to the last lateral segment of the previous year. The current $L_{\text {SLast }}$ is estimated as

$$
L_{S L a s t}=L_{B(y+1)}-L_{S F i r s t}-\left(N_{B L a t}-1\right) \cdot L_{I B}
$$

This ensures that that the total length growth $\Delta L_{\mathrm{B}}$ equals the sum of the lengths of all new segments. To estimate the diameter of these branch segments $D_{\mathrm{S}}$, Eq. 4.44 can be applied.

After the length and diameter of all new first order branch segments has been calculated, the length and diameter of all new lateral second order branches are estimated. For this, since the total length growth of the first order branch $\Delta L_{\mathrm{B}}$ is known, we first calculate the cross-sectional area $A_{\text {Sec }}$ of the branch section representing this growth (Fig. 4.6c).

$$
A_{S e c}=\left(\frac{D_{T(y+1)}}{2} \cdot \frac{L_{T(y+1)}-L_{T(y 0)}}{L_{T(y+1)}}\right)^{2} \cdot \pi
$$

In compliance with the pipe model theory, the sum of the cross-sectional areas of all new lateral branches is assumed to equal $A_{\text {Sec }}$. $A_{\text {Sec }}$ is thus equally divided between all new lateral branches. This also means that we assume that all new lateral branches have the same size, i.e. we do not explicitly consider effects such as acrotony or mesotony. With $n_{\text {BLat }}$, the diameter of each new lateral branch $D_{\text {BLat }}$ is calculated as follows.

$$
D_{B L a t}=2 \cdot \sqrt{\frac{A_{S e c}}{n_{B L a t} \cdot \pi}}
$$

Finally, the length of each new lateral second order branch $L_{\mathrm{B}}$ is calculated based on the speciesspecific allometric diameter-length relationship (Eq. 4.7). We have to remember that branches are represented at two scales, and thus for each new branch, the total length and diameter as well as the segment lengths and diameters have to be calculated. As these new second order branches consist of a single segment, $L_{\mathrm{S}}=L_{\mathrm{B}}$ and $D_{\mathrm{S}}=D_{\mathrm{B}}=D_{\text {BLat }}$. 
(a)

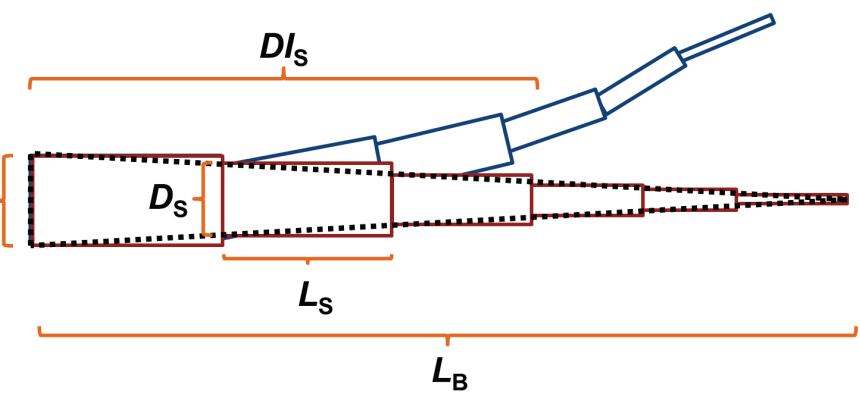

(b)

y0:
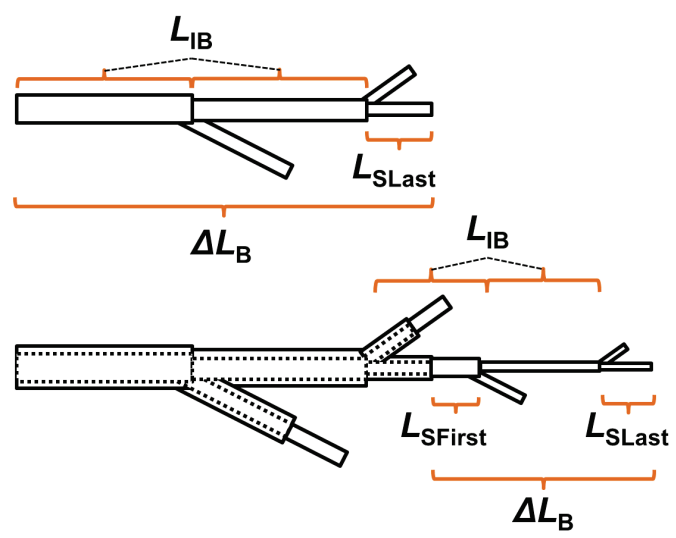

(c)

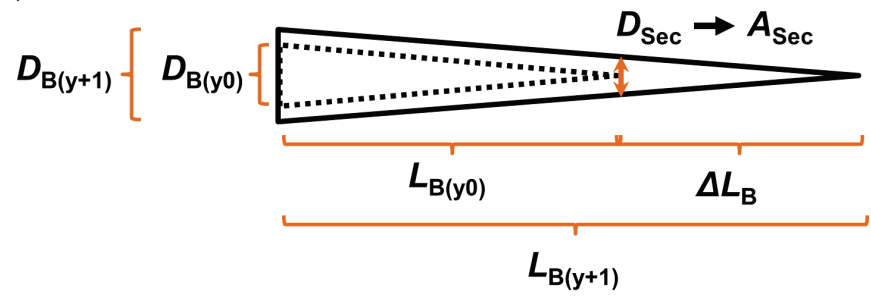

Figure 4.6. Illustration of structural variables. (a) Branches are represented at two different scales. At the coarse scale, branches are described by their total length $L_{\mathrm{B}}$ and diameter $D_{\mathrm{B}}$, while at the fine scale they are described as a collection of topologically connected smaller branch segments (length $L \mathrm{~s}$, diameter $D \mathrm{~s}$ ), which are visually represented by $3 \mathrm{D}$ cylinders (here: $2 \mathrm{D}$ representation). The distance of each branch segment to the branch base $D I_{\mathrm{s}}$, which is exemplarily shown for the fourth branch segment, determines the diameter of the branch segment $D$ s. The fine-scale representation of branches allows a more realistic irregular visualization (blue colored branch as example). (b) Branch development in two successive years $(\mathrm{y} 0$ and $\mathrm{y}+1)$. The upper panel shows a newly created 1 st order branch with lateral second order branch segments. The variable internode length $\mathrm{L}_{\mathrm{IB}}$ defines the length of the first two branch segments and the branching points between first and second order branches. Since the total length growth $\Delta L_{\mathrm{B}}$ is not an integer multiple of the internode length $\mathrm{L}_{\mathrm{IB}}$, the length of the last lateral segment $\mathrm{L}_{\text {SLast }}$ differs from $\mathrm{L}_{\mathrm{IB}}$. The lower panel shows the further development of this branch in the next time step. The internode length $\mathrm{L}_{\mathrm{IB}}$ in this time step may vary from that in the previous step, and because a shorter later lateral branch segment exists, and additional segment with a length of $\mathrm{L}_{\text {SFirst }}$ is inserted so that distance between the branching points equals $\mathrm{L}_{\mathrm{IB}}$. (c) Branch growth at the coarse scale. Based on the diameter and length growth in one time step, the cross-sectional area A $\mathrm{sec}_{\text {of }}$ the branch section representing the current length growth $\Delta \mathrm{L}_{\mathrm{B}}$ can be calculated. A $\mathrm{S}_{\text {sec }}$ is used to estimate the diameter of lateral branches.

So far, we have demonstrated how to calculate the state variables of branches at both scales when branches grow in length. However, when trunks grow in length, new lateral first order branches may establish, which, in turn, may ramify into second order branches. Trunks are not divided into separate segments, but nonetheless the internode length between two branching points at the trunk 


\section{Functional-structural forest model}

is an important information. The species-specific internode length of the trunk can be specified separately (minimum $L_{\text {ITMin }}$, maximum $L_{\text {ITMax }}$ ), and the method to calculate the variable internode length of trunks $L_{\mathrm{IT}}$ corresponds to that for branches (Eq. 4.45). $L_{\mathrm{IT}}$ thus defines the position at the trunk where to attach the new first order branch. When applying the methods describe above (Eqs. 4.43-4.50) in a recursive manner, all essential state variables of this first order branch and attached second order branches can be calculated.

The last step remaining is to update the diameter of all branch segments that already existed, which is done by applying Eq. 4.44. Obviously, the length of existing branch segments does not change.

After the structure of all woody tree components has been simulated, the remaining tree components, namely apical meristems and leaf compartments, need to be considered. Branches and trunks are always terminated by an apical meristem, and thus the structural growth simulations in GroIMP are carried out in a ways that this condition is true at all times. Each meristem is re-associated with the voxel in which it is located after the tree structure has been updated.

As a result of structural growth, new second order branches may be generated, and/or existing second order branches may grow into adjacent voxels. In these cases, new leaf compartments are associated with these branches and the initial leaf biomass is specified. We assume that the newly generated branches or branch sections consist entirely of sapwood and consequently, following the pipe model theory, their cross-sectional area and the leaf area of the associated leaf compartments are correlated via the parameter $L P_{\text {ratio }}$. For new second order branches, the crosssectional area $A_{\mathrm{B}}$ can thus be estimated from their known diameter, while for second order branches that increased in length, the cross-sectional area representing this growth $A_{\text {Sec }}$ can be calculated based on Eq. 4.49. Based on the cross-sectional area $\left(A_{\mathrm{B}}\right.$ or $\left.A_{\mathrm{Sec}}\right)$ and the specific leaf area $S L A$, the total leaf area $A_{\text {LSum }}$ associated with the branch/branch section can be estimated.

$$
A_{L S u m}=\frac{A_{B} \cdot L P_{\text {ratio }}}{S L A}
$$

For simplicity, we assume that $A_{\text {LSum }}$ is evenly divided among all new leaf compartments. For this, all new voxels a branch is intersecting with are estimated and in each voxel a new leaf compartment with the initial leaf biomass $B_{\text {LInit }}$ is generated. 


$$
B_{\text {LInit }}=\frac{A_{L S u m}}{n_{V}}
$$

where $n_{\mathrm{V}}$ is the number of new voxels a branch is intersecting with.

Structural growth also includes the loss of existing tree components. Leaf compartments are lost if they no longer contain leaves (i.e. leaf biomass is zero). However, since the leaf biomass is simulated using an exponential function (Eq. 4.34), it would only converge to, but never reach zero, if the exponent is negative. Thus, we defined that leaf compartments are removed when the leaf biomass drops below a minimum threshold $B_{\mathrm{LMin}}$. This threshold can be understood as the biomass of one leaf; the last leaf is thus dropped if $B_{\mathrm{L}}<B_{\mathrm{LMin}}$. If a leaf compartment is removed, it cannot be reestablished. This means that such leafless parts of a branch do not contain resting meristems.

Branches are shed if they lost all associated leaf compartments. This also implies that first order branches are shed when all connected second order branches are shed. Apart from this physiologically determined branch turnover, we also integrated the option to remove branches based on disturbances or mechanical stress. Branches may either be randomly removed (BrMortMethod $=1)$ or based on their biomass (BrMortMethod=2). In the first case the branch mortality rate $m_{\mathrm{BR}}$ defines the chance of a branch to be removed randomly at each time step, in the second case the branch mortality rate $m_{\mathrm{B}}$ is calculated as follows.

$$
m_{B}=m_{B B} \cdot\left(\frac{1}{3} \cdot \pi \cdot D_{B} \cdot L_{B} \cdot \rho_{W}\right)^{-M_{B S}}
$$

where $m_{\mathrm{BB}}$ is the biomass-based branch mortality rate, and the product within brackets is the mass of the branch, which is calculated by its diameter $D_{\mathrm{B}}$, length $L_{\mathrm{B}}$ and wood density $\rho_{\mathrm{W}}$ assuming that it is cone-shaped. $M_{\mathrm{BS}}$ is a scaling factor describing the decrease in mortality rate with increasing biomass (negative exponent). According to the metabolic theory, this scaling factor is assumed to be close to $1 / 4$ regarding the mortality of entire trees (Brown et al. 2004; Muller-Landau et al. 2006b). However, the scaling factor for branches may be site-specific and thus we integrated it as freely definable variable. Nevertheless, the user can choose to simulate only physiologicallydetermined branch fall (BrMortMethod $=0$ ).

In the previous description of structural growth, the structural traits were mentioned only briefly. The structural traits define how the different tree segments are spatially arranged, and thus they are required for a sufficient representation of the visible 3D tree structure. It was our intention to specify a minimal set of structural traits capable of reproducing the most obvious differences in 


\section{Functional-structural forest model}

tree structure observed in nature (Table 4.1; Fig. 4.7). The main functional traits and concepts are described hereafter.

The trunk is the orthotropic axis in each tree. First order branches are plagiotropic shoots that show a radial symmetry around the trunk. The angle between successive first order branches, seen from the bird's eye view, is calculated based on $P h_{\mathrm{FO}}$ which defines how many first order branches are arranged within a complete $360^{\circ}$ circle (Fig. $4.7 \mathrm{a}$ ). Thus, the angle between two successive first order branches $\alpha_{\mathrm{TFO}}$ is calculated as

$$
\alpha_{T F O}=\frac{360}{P h_{F O}}
$$

After a complete $360^{\circ}$ circle, the successive first order branch is generated at an angle of $1 / 2$ times $\alpha_{\mathrm{TFO}}$ after its predecessor. This ensures that the branches do not directly shade the branches below. The angle of first order branches seen from the side is defined by $\alpha_{\mathrm{SFO}}$ (Fig. $4.7 \mathrm{~b}$ ). In contrast to the first order branches, we assume that second order branches do not show a radial but rather a dorsiventral symmetry, i.e. they are arranged in the same plane as their mother branch. Their branching angle relative to the mother branch is defined by $\alpha_{\text {TSO }}$ (Fig. 4.7c). For simplicity, second order branches are always arranged in an alternating manner. As mentioned, the model differentiates between the internode lengths of branches $L_{\mathrm{IB}}$ and trunks $L_{\mathrm{IT}}$. The actual internode length at a given time step depends on the total growth of the specific branch or trunk and varies between the minimum ( $\left.L_{\text {IBMin }}, L_{\text {ITMin }}\right)$ and maximum internode lengths ( $\left.L_{\text {IBMax }}, L_{\text {ITMax }}\right)$, which are species-specific structural traits. Gravitropism or phototropism is often observed in trees: branches may bend downwards due to gravity and/or upwards to the sun. The strength of tropism $S_{\text {Trop }}$ is an additional functional trait, whereby negative values represent phototropism and positive values represent gravitropism. 
(a)

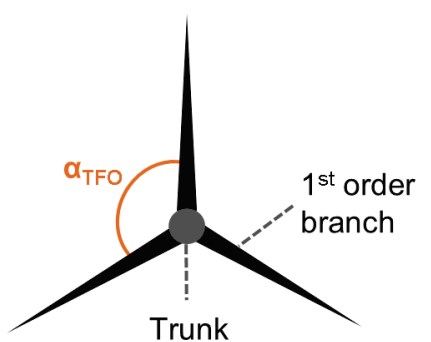

(b) Side view on tree

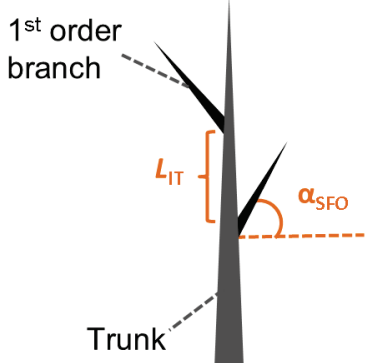

(c) Top view on $1^{\text {st }}$ order branch

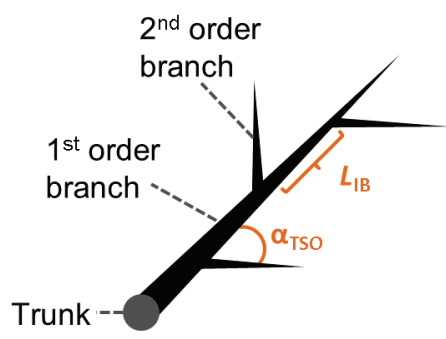

Figure 4.7. Illustration of the main structural traits. (a) Top view on tree showing the main trunk and first order branches. The angle between two consecutive first order branches is given by $\alpha_{\text {TFO }}$. (b) Side view on tree showing main trunk and first order branches. The trunk internode length $L_{\mathrm{IT}}$ and the angle of the first order branch relative to the horizontal plane $\alpha_{\text {SFO }}$ define the coarse structure of the tree (c) Top view on tree showing one first and three second order branches. The branch internode length $L_{\mathrm{IB}}$ and the angle between first order branch and second order branch $\alpha_{\mathrm{TSO}}$ define the fine branching structure of the tree.

Differences in the mentioned structural traits create a variety of different tree structures. However, if a tree grows according to its structural traits in a deterministic manner, the resulting tree becomes too symmetrical. Thus, our model allows activating stochastic variation of structural traits if more realistic structures are desired (Stochasticity=0: deterministic growth, Stochasticity=1: stochastic growth). If stochastic growth is chosen, trees can deviate from their intrinsic structural growth pattern, whereby the strength of the random deviation is defined by a set of additional structural traits defining the maximum deviation of a specific species. For instance, the maximum deviation from $\alpha_{\mathrm{TSO}}$ is given by $S t_{\alpha \mathrm{TSO}}$ and, in this case, the branching angle may thus vary within $\alpha_{\mathrm{TSO}} \pm S t_{\alpha \mathrm{TSO}}$. The 'stochastic' structural traits can be understood as additional characteristic of species defining their structural irregularity. Such traits can be defined for all angles ( $\left.S t_{\alpha \mathrm{TSO}}, S t_{\alpha \mathrm{TFO}}, S t_{\alpha \mathrm{SFO}}\right)$ and the tropism strength $\left(S t_{\mathrm{Trop}}\right)$. In addition, $S t_{\mathrm{TW}}$ specifies the strength of branch twisting. This means that each time a new branch segment is generated, it may deviate from the direction of its predecessor by the three axis in space (head, left, up), whereby the maximal rotation along each axis is given by $S t_{\mathrm{TW}}$.

\section{Tree mortality}

The metabolic theory of ecology generally predicts natural mortality rates to scale with biomass as the negative $1 / 4$ power (McCoy \& Gillooly 2008). Muller-Landau et al. (2006) tested this scaling relationship based on data from 10 old-growth tropical forest. They found large differences in the scaling factors between forest sites, which were mostly inconsistent with metabolic theory. However, at all sites the mortality rates consistently decreased with size when considering small trees $<20 \mathrm{~cm}$ diameter. For the larger trees, this trend differed and sometimes even reversed, i.e. 


\section{Functional-structural forest model}

the mortality rate of larger tree increased at some forest sites. Muller-Landau et al. (2006) argued that there are additional site-specific mechanisms not explicitly considered in the metabolic theory.

In this model, there is only one explicit cause of mortality that directly emerges from the model approach, which is mortality due to carbon starvation. This happens when a tree has lost all its leaf compartments. The probability to lose a leaf compartment due to a negative carbon budget is higher in the dark understory compared to the upper forest zones. Therefore, the likelihood that a tree dies due to carbon starvation is higher for smaller trees and commonly decreases with size, which agrees with the pattern for small trees observed by Muller-Landau et al. (2006). However, large trees growing in the canopy can also die due to carbon starvation in this model. When a tree grows close to its maximum height, it enters a phase of senescence where it loses more leaf biomass than it can produce, which ultimately leads to the loss of all leaf compartments and thus to carbon-based mortality. Consequently, the mortality rate due to carbon starvation might increase also for larger trees, explaining the trends observed by Muller-Landau et al. (2006).

Nevertheless, it is unlikely that mortality due to carbon starvation is sufficient to capture all mortality mechanisms. We thus additionally integrated a mass-dependent mortality rate to account for additional causes of tree mortality, such as infections by pathogens or severe physical damages, which should scale with size. Due to the observed uncertainties in the scaling factor (Muller-Landau et al. 2006b), we integrated it as a free parameter $M_{\mathrm{TS}}$. The biomass-dependent mortality rate $m_{\mathrm{T}}$ is thus calculated as follows:

$$
m_{T}=m_{T B} \cdot\left(\frac{1}{3} \cdot \pi \cdot D_{T} \cdot L_{T} \cdot \rho_{W}\right)^{-M_{T S}}
$$

where $m_{\mathrm{TB}}$ is the biomass-based tree rate, and the product in the bracket is the mass of the tree trunk, which is calculated by its diameter $D_{\mathrm{T}}$, length $L_{\mathrm{T}}$ and wood density $\rho_{\mathrm{W}}$ assuming that it is cone-shaped. This equation quantifies the probability of each tree to die, which decreases with biomass.

We further integrated the option to simulate mortality due to extrinsic factors, such as disturbances or gap formation. If the user intends to simulate disturbance events (TrMortDist=1), the average number of years between two events $F_{\text {Dist }}$ and the probability of the disturbance-mediated mortality $m_{\text {Dist }}$ are defined. If a direct effect of falling trees on neighboring trees mimicking gap formation shall be simulated (TrMortNeigh $=1$ ), the parameters $m_{\text {Neigh }}$ and $D_{\mathrm{NMin}}$ have to be defined. We assume that only larger trees cause surrounding trees to break and die, and the minimum diameter of falling trees to be considered is given by $D_{\mathrm{NMin}}$. The crown radius $C R_{\mathrm{r}}$ of 
the falling trees defines the gap size, i.e. all smaller trees within distance $C R_{\mathrm{r}}$ to the falling trees may die with a probability of $m_{\text {Neigh }}$. Tree mortality is the last submodel, thereafter the model proceeds with the next time step.

\section{External model control, export and visualization}

This model is designed to be flexible and controlled by the user via simple text files. This allows manipulation and customization for simulation experiments without source code changes. There are two different types of text files, the global and the pass files.

The global file contains a set of parameters defining the basic set up of the model (Table C.2). This includes the general decision whether a forest stand or an individual tree shall be simulated, the spatial extent and resolution of the model space, the number of time steps and the number of replicates. Furthermore, the time intervals in which different types of model results are saved can be determined. The state variables of the tree components constitute the model results at the lowest hierarchical level, based on which higher level results are calculated. Users interested only in higher-level results can choose not to save the low-level results, or to save them in greater time intervals, by this reducing the required hard disk space (a 1 ha forest stand may consist of several millions branch segments). There are a total of six different types of result files: Shoots: state variable of tree components, Trees: tree level results, Forest: forest level results, Species: species pool, Voxels: leaf biomass and light in voxels, Mortality: number and causes of tree mortality. Which specific variable are saved in each of these files can be seen in Table C.3. A short overview on important results is given in Fig. 4.8. 
(a)

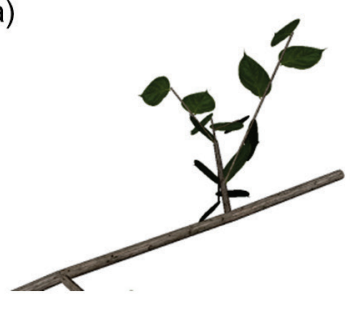

- $3 D$ position of tree components

- Length and diameter of branch segment

- Length and diameter of trunk

- Leaf biomass in leaf compartments (b)

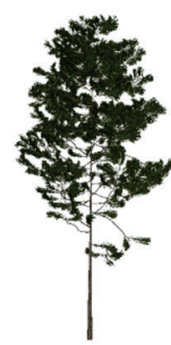

- Tree height

- Tree DBH

- Crown width

- Crown depth

- Crown area

- Woody biomass

- Leaf biomass

- Net-primary production

- NPP of leaves

- NPP of trunks/branches (c)

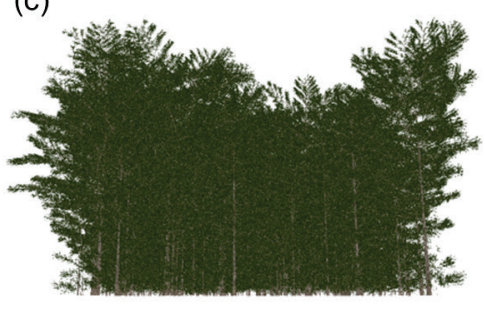

- Number of stems

- Basal area

- Above-ground biomass

- Canopy height

- Leaf area index

- Total NPP

- Canopy NPP

- Stem turnover

- $\quad A G B$ residence time

Figure 4.8. Exportable model results at the three hierarchical scales: (a) tree components, (b) individual trees and (c) forest stands. This model allows saving model results as text files, and examples of important exportable variables are shown. A complete list of all variable is provided in Table C.3.

Visual control of simulated trees or forests is an important additional method to evaluate the quality of the model. Therefore, a picture showing the tree/forest structure is saved to disk at each time step. The perspective from which the picture is taken can be configured in GroIMP. Two general methods how trees are visualized are implemented, and they can be specified in the global file. First, trees can be represented by their woody components only (VisualizationShader $=0$ ), whereby second order branches connected to leaf compartments can be colored according to the state of the leaf compartment (Fig. 4.9). Second, trees can be represented by woody components and leaves (VisualizationShader=1). In our model we are not simulating individual leaves, however, for aesthetic purposed we integrated a technique which allows visually representing leaf compartments by leaf shaders (this technique is used in Fig. 4.8). 
(a)

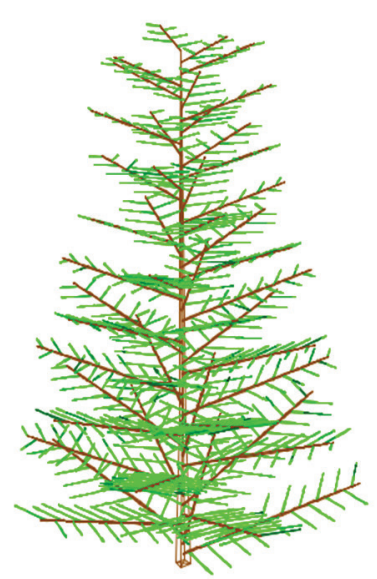

(b)

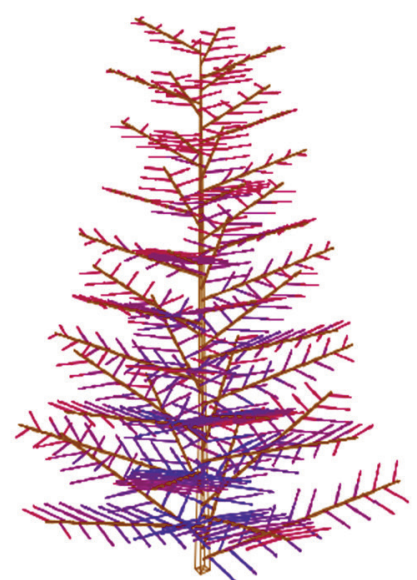

(c)

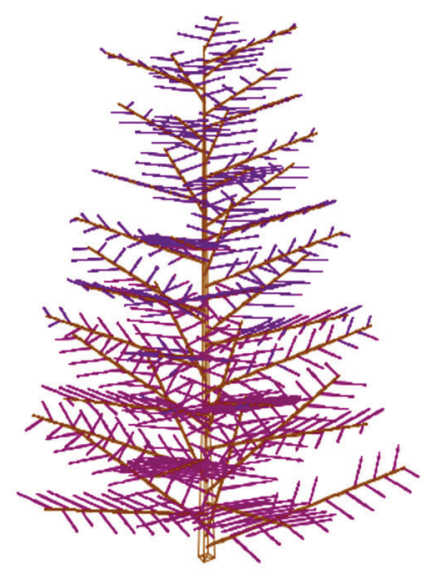

Figure 4.9. Visual representation of trees. If a wireframe model is chosen (VisualizationShader $=0$ ), three different methods to represent the leaf compartments attached to second order branches can be specified: (a) Second order branches are colored in different shades of green depending on the associated leaf biomass (VisualizationMethod $=0$ ). (b) Second order branches are colored according to the light conditions at the leaf compartments, with red colors representing high light intensities (VisualizationMethod=1). (c) Second order branches are colored according to the net carbon assimilation in the leaf compartments, with red colors representing higher values (VisualizationMethod=2). If a rendered representation is chosen (VisualizationShader $=1$ ), leaves representing the leaf compartments are visualized (see Fig. 4.8)

The pass files contain a set of parameters for each replicate, which means that the number of replicates specified in the global file and the number of pass files must be equal. Each pass file includes global parameters, ranges of functional and structural traits, but also parameters to switch on and off optional model mechanisms. An exhaustive list of all parameters in the pass file is available in the Supporting Information (Table C.4).

\subsubsection{Model parameterization and validation}

We used the key ideas of the pattern-oriented modeling (POM) framework to parameterize and validate our model (Grimm et al. 2005; Grimm \& Railsback 2011). The rationale behind POM is to reduce the uncertainty in model structure and parameters by comparing model results with multiple biological/ecological patterns at different hierarchical levels and scales, leading to more comprehensive and rigorous bottom-up models (Grimm et al. 2005). As our model aimed to reproduce long-term tropical forest dynamics, as well as realistic 3D growth patterns of the different functional tree types during their entire life cycles, we included multiple patterns at the forest and the tree level. This set of patterns comprised both quantitative and rather qualitative patterns, which makes it difficult to aggregate all patterns in a single comprehensive criterion evaluating model performance. We thus applied a three-step POM evaluation strategy and consecutively evaluated the performance of our model in reproducing i) the general structure and 


\section{Functional-structural forest model}

dynamics of tropical forests, ii) complex patterns at the forest level and iii) life-history growth patterns of different functional tree types.

In a first step, we evaluated the performance of our model in reproducing the general structure and dynamics of tropical forests. Pantropical studies have shown substantial differences in forest characteristics between continents and regions (e.g. Feldpausch et al. 2011), and in this study we focused on the well-studied Neotropical lowland forests. While typical ranges of attributes of such mature lowland forests, such as the basal area or net primary production per hectare, are relatively well-known, long-term field data to which the model could be fitted are still scarce. Taking into account this limitation, we estimated ranges of 12 important attributes characterizing both forest structure and dynamics based on a literature review (see Table 4.3; Table C.5). We excluded data from rather extreme or recently disturbed sites; the estimated ranges should be representative for average lowland forests in dynamic equilibrium state. Model performance was assessed by simulating 0.25 ha forest plots starting from bare ground for 500 years, and calculating the model performance criterion $a_{\mathrm{M}}$ that tests if the attributes of the simulated forest in dynamic equilibrium state are within the empirical ranges:

$$
\alpha_{M}=\frac{\sum_{i}^{n}\left(\frac{\sum_{t \min }^{t m a x} \beta_{i, t}}{t \max -t \min }\right)}{n}
$$

where $t_{\min }$ is the time after which an equilibrium state is expected (here, $t_{\min }=200$ years), $t_{\max }$ is the total number of years simulated (here, $t_{\max }=500$ ), $n$ is the total number of forest attributes (here, $\mathrm{n}=12$ ) and $\beta_{\mathrm{i}, \mathrm{t}}$ is a Boolean variable describing for each attribute at each time step if the attribute value is within the estimated range $\left(\beta_{\mathrm{i}, \mathrm{t}}=1\right)$ or not $\left(\beta_{\mathrm{i}, \mathrm{t}}=0\right)$. The optimal values of $a_{\mathrm{M}}=1$ can be reached if all attributes of the simulated forest are within the estimated ranges continuously from $t_{\min }$ to $t_{\max }$. This approach assumes that a sufficiently stable equilibrium state is modelled, and the time to reach this state when starting from bare ground is thus a secondary prediction we additionally considered as validation criterion.

In a second step, we evaluated whether our model adequately reproduced more complex patterns at the forest level. This step mainly involved qualitative comparisons between simulations and observations based on visualized patterns. The following patterns were considered: (i) Crown architectures of trees in stands usually change markedly with tree height, and while the crown area usually shows a clear non-linear allometric relationship with height, crown width and branching height are commonly rather linearly correlated with height (Alves \& Santos 2002; Iida et al. 2011). (ii) The vertical leaf area density in undisturbed forests within stands often peaks in 
the canopy zone, sometimes with an additional peak in the understory (Stark et al. 2012; Taubert et al. 2015). (iii) The height-diameter relationship is a typical characteristic of forest and for the Neotropics, this relationship was best described using a three-parameter exponential equation with an asymptotic maximum height of $38.8 \mathrm{~m}$ (Banin et al. 2012). (iv) The frequency distributions of tree diameter, height and age is typically right-skewed when considering all trees in a stand (Campbell et al. 1986; Worbes et al. 2003). When considering only trees above $10 \mathrm{~cm}$ in diameter at breast height (DBH), a normal or slightly right-skewed distribution is commonly observed (Oliveira-Filho et al. 1994; Worbes et al. 2003). In addition, the metabolic theory of ecology predicts a linear decrease in stem diameter frequency on a log-log-scale, but observations revealed deviations from this predictions for larger tree diameters (Muller-Landau et al. 2006b). To visualize and compare the mentioned patterns, we used simulated data after reaching dynamic equilibrium state in intervals of 50 years to avoid temporal autocorrelation (More details are also provided in the figure captions in the Results section).

In a third step, we evaluated simulated growth of individual trees under controlled conditions. 3D tree growth patterns in our model emerge from processes at lower-hierarchical levels, and a visual analysis of ontogenetic tree growth trajectories in combination with an analysis of the changes in height and diameter growth, biomass and productivity during tree ontogeny served as fist indicator for the structural realism of our tree growth model. Furthermore, we systematically analyzed how changes in species traits (SLA, wood density) and environmental conditions (light, SI) influence tree growth. To cover the natural trait and environmental space in tropical forests, we altered each of these factors within their natural ranges while keeping the other factors constant at medium levels. Due to the size and longevity of trees, controlled experiments on changes in growth rates and morphology over a tree's lifespan are practically impossible, and consequently data equivalent to our simulation experiment is missing. Nevertheless, based on numerous field and theoretical studies, we have a fairly good understanding of some qualitative and quantitative patterns during tree ontogeny. For instance, while the height growth of undisturbed trees is expected to continue at decreasing rates until reaching maximum height, the diameter growth rates tend to peak at a certain height or age (Clark \& Clark 1999). In addition, the ranges of diameter growth rates, maximum tree heights and partly also maximum ages in tropical forest are well-studied (e.g. Martínez-Ramos \& Alvarez-Buylla 1998; Clark \& Clark 2001; Chao et al. 2008). Life-history variations among trees have furthermore been associated with different functional traits (Poorter \& Bongers 2006; Iida et al. 2012). Based on such observations, we evaluated our tree growth model mostly qualitatively.

To find an optimal parameter combination following the described three-step POM evaluation strategy, suitable parameter ranges were defined at first. Many parameters have natural ranges, 
which were estimated based on literature values (Table C.6). The parameters can be categorized into 4 classes: parameters specifying the i) environmental conditions, ii) functional and iii) structural traits, and iv) global model parameters. For the traits, minima and maxima have to be defined. For most traits, suitable ranges can be defined from observations. For example, wood density and SLA are well-studied (e.g. Baker et al. 2004; Patiño et al. 2012), and consequently the observed range of these traits define the suitable ranges in the model. Likewise, most structural traits are easy-to-interpret characteristics of tree structure, such as branching angles, and their minima and maxima can be determined based on empirical knowledge. This means that most free parameters belong to the class of global model parameters (Table C.6). After suitable ranges were defined, the best parameter set was attained using a manual parameterization strategy as despite optimizations, model run times were too long for automatic parameterization. This means that model parameters were repeatedly adjusted and analyzed until yielding an appropriate set of parameter values. 
Table 4.3. Typical ranges of forest attributes in Neotropical lowland forests derived from a review of the literature. We concentrated on reviews covering multiple forest plots or larger forest areas. More details are given in Table C.5.

\begin{tabular}{|c|c|c|c|}
\hline Forest attribute & Unit & Typical range & References \\
\hline Stem density $(>10 \mathrm{~cm})$ & $\mathrm{ha}^{-1}$ & $480-620$ & $\begin{array}{l}\text { Feldpausch et al. (2011), Banin et al. } \\
\text { (2012) }\end{array}$ \\
\hline Stem density & $\mathrm{ha}^{-1}$ & $2500-5000$ & $\begin{array}{l}\text { Girardin et al. (2013), Chave et al. (2003), } \\
\text { DeWalt \& Chave (2004) }\end{array}$ \\
\hline Basal area & $\mathrm{m}^{2} \mathrm{ha}^{-1}$ & $25-35$ & $\begin{array}{l}\text { Feldpausch et al. (2011), Banin et al. } \\
\text { (2012), Girardin et al. (2013), Malhi et al. } \\
\text { (2006), Baker } \text { et al. (2004) }\end{array}$ \\
\hline Above-ground biomass & $\mathrm{Mg} \mathrm{ha}^{-1}$ & $250-350$ & $\begin{array}{l}\text { Malhi et al. (2006), Feldpausch et al. } \\
\text { (2012), Baker } \text { et al. (2004) }\end{array}$ \\
\hline Canopy height & $\mathrm{m}$ & $27-38$ & $\begin{array}{l}\text { Feldpausch et al. (2011), Asner et al. } \\
\text { (2013), Girardin et al. (2013) }\end{array}$ \\
\hline Mean DBH $(>10 \mathrm{~cm})$ & $\mathrm{m}$ & $0.18-0.26$ & $\begin{array}{l}\text { Lieberman et al. (1996), Banin et al. } \\
\text { (2014), Sawada et al. (2015) }\end{array}$ \\
\hline Leaf area index & $\mathrm{m} \mathrm{m}^{-2}$ & $3.5-5.5$ & $\begin{array}{l}\text { Myneni et al. (2007), Doughty \& Goulden } \\
\text { (2008), Caldararu, Palmer \& Purves (2012) }\end{array}$ \\
\hline Total NPP & $\operatorname{Mg~ha}^{-1} a^{-1}$ & $10-20$ & $\begin{array}{l}\text { Malhi, Doughty \& Galbraith (2011), } \\
\text { Aragão et al. (2009), Malhi et al. (2013) }\end{array}$ \\
\hline Canopy NPP & $\operatorname{Mg~ha}^{-1} a^{-1}$ & $5-10$ & $\begin{array}{l}\text { Chave et al. (2010), Malhi et al. (2013), } \\
\text { Aragão et al. (2009) }\end{array}$ \\
\hline Basal area growth & $\mathrm{m}^{2} \mathrm{ha}^{-1} \mathrm{a}^{-1}$ & $0.5-1.0$ & $\begin{array}{l}\text { Malhi et al. (2004), Lewis et al. (2004b), } \\
\text { Banin et al. (2014) }\end{array}$ \\
\hline AGB residence time & $\mathrm{a}$ & $25-60$ & $\begin{array}{l}\text { Malhi et al. (2011, 2015), Galbraith et al. } \\
\text { (2014) }\end{array}$ \\
\hline Stem turnover $(>10 \mathrm{~cm})$ & $a^{-1}$ & $1-4$ & $\begin{array}{l}\text { Quesada et al. (2012), Lewis et al. (2004), } \\
\text { Malhi et al. (2009) }\end{array}$ \\
\hline
\end{tabular}




\section{Functional-structural forest model}

\subsection{Results}

Following the 3-step POM validation strategy we found a parameter combination that resulted in realistic model behavior at the forest and the tree level (see Table C.4 for the optimized parameter set). Starting from bare ground, the simulated forest increased in stem number, above-ground biomass, and basal area and reached a dynamic equilibrium state after ca. 80-100 years (Fig. 4.10). In this equilibrium state, all 12 monitored forest attributes were within the ranges typically observed for Neotropical forest for the rest of the simulated 500 years, i.e. the validation criterion attained its optimal values of $\alpha_{M}=1$ (see 1 ha stand result in Fig. 4.10). Fluctuations around the equilibrium increased with decreasing stand size as the relative effects of gap-creating mortality events were stronger at smaller scales (Fig. 4.10).

Considering all stems, the frequency distributions of tree diameter, height, and age were rightskewed (Fig. 4.11). When only considering stems $>10 \mathrm{~cm}$ in DBH, the height and age distribution were rather normally distributed (Fig. $4.11 \mathrm{a}, \mathrm{c}$ ). On log-scale, tree numbers decreases almost linearly with diameter and age, with deviations from this pattern only at large size/age classes. In contrast, the tree height distribution showed a distinct hump between 25 and $35 \mathrm{~m}$ (Fig. 4.11b). On log-log-scale, tree height distribution was linear for small individuals but became curvilinear at larger diameters (Fig. 4.11d). Trees reached a maximum diameter of $\sim 100 \mathrm{~cm}$, a maximum height of $\sim 50 \mathrm{~m}$ and a maximum age of $\sim 250$ years.

Crown architectures within the forest stand changed significantly with tree height (Fig. 4.12). We found a positive linear relationship between tree height and crown width (Fig. 4.12b), and an exponential relationship between tree height and branching height (Fig. 4.12a), as well as between tree height and crown area (Fig. 4.12c). However, due to differences in functional and structural traits of the trees, as well as in their ontogenetic stages, there was substantial scatter around the average trends. 

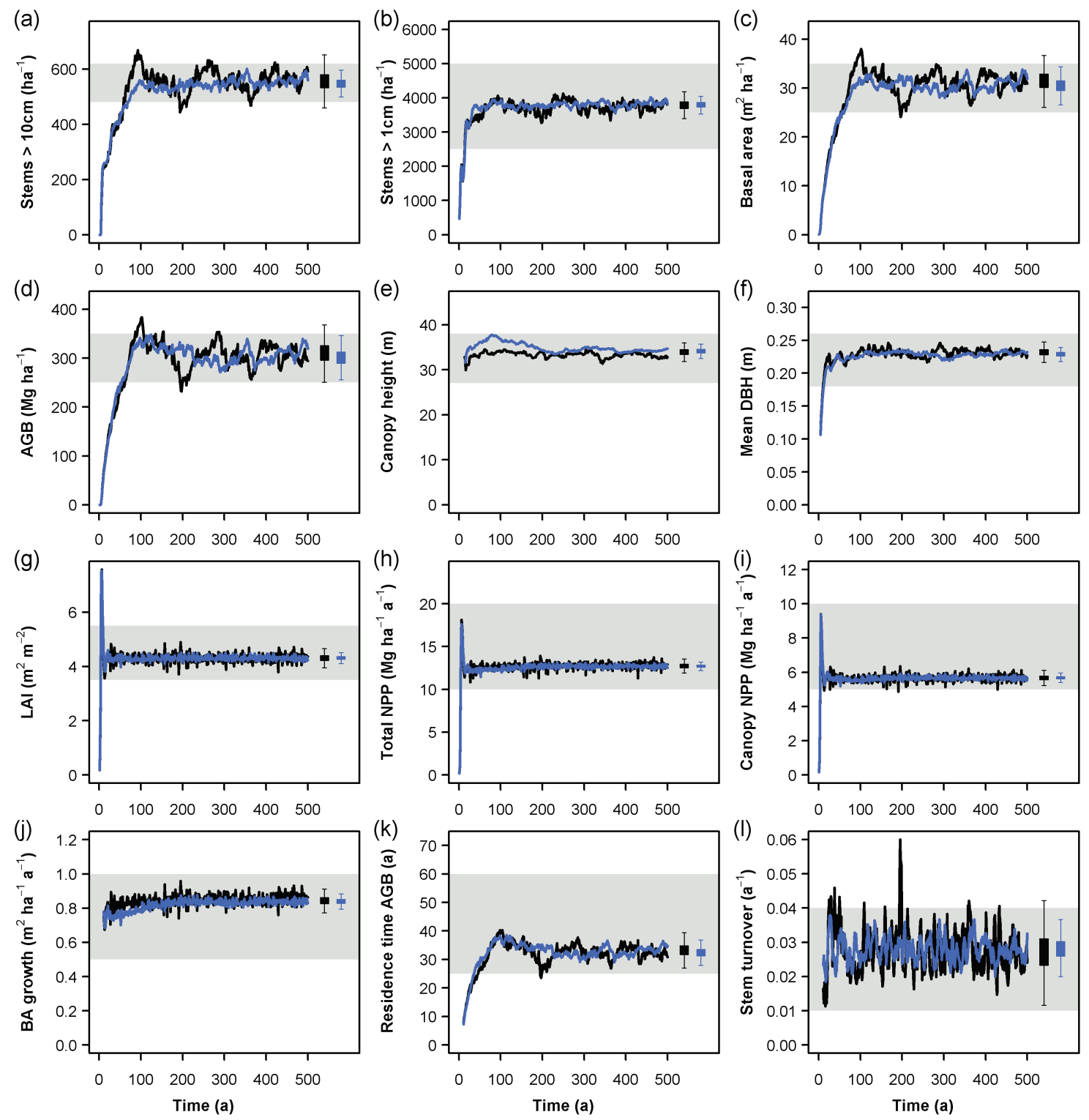

Spatial extent of forest stand: $\quad-50 \times 50 \mathrm{~m} \quad-100 \times 100 \mathrm{~m} \quad$ Literature range

Figure 4.10. Simulated long-term forest dynamics. (a) Stem density of all stems with DBH $>10 \mathrm{~cm}$, (b) Stem density of all stems with DBH $>1 \mathrm{~cm}$, (c) Basal area, (d) Above-ground biomass (ABG), (e) Canopy height (mean height of all trees $>40 \mathrm{~cm}$ in DBH), (f) Mean DBH of all stems $>10 \mathrm{~cm}$ in DBH, (g) Leaf area index (LAI), (h) Total aboveground net primary production (NPP), (i) Canopy net primary production (NPP of leaves and second order branches), (j) Basal area (BA) growth, (k) Residence time of above-ground biomass, (l) Turnover of all stems $>10 \mathrm{~cm}$ in DBH. Black lines represent simulations at 0.25 ha scale, and blue line at 1 ha scale. The grey-shaded areas indicate typical ranges for each forest attribute in Neotropical forests (see Table 4.3) Boxplots show interquartile ranges (boxes) and approximate $95 \%$ confidence intervals (whiskers) of the forest attributes in dynamic equilibrium state, i.e. from years $150-500$, based on 5 replicates of the forest model. 


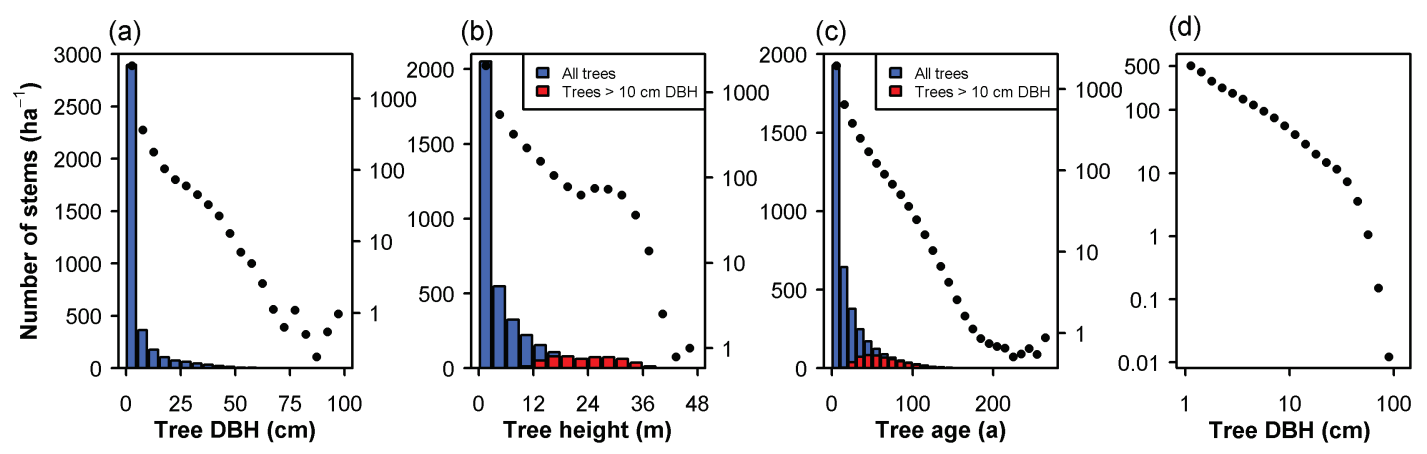

Figure 4.11. Frequency distributions: (a) tree DBH, (b) tree height, and (c) tree age. Distributions are shown on normal (colored bars, left axes) and log-scale (black dots, right axes). The average frequency in each size class over the years 150-500 (equilibrium state) is shown here. (d) Tree DBH distribution on log-log scale. Values in each size class were binned to the class width.
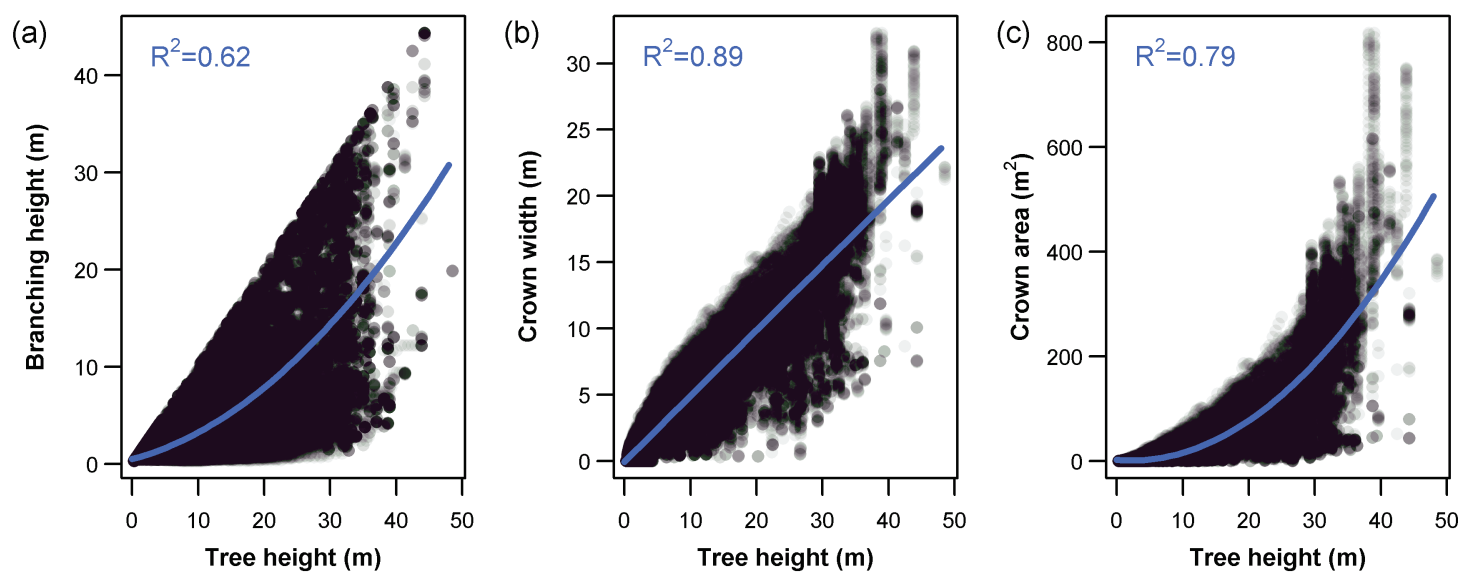

Figure 4.12. Relationship between tree height and crown parameters: (a) branching height (height of lowest first order branch), (b) crown width and (c) crown area. Each dot represents a single tree in the simulated forest stand. To reduce the degree of temporal pseudoreplication, all trees in the forest stand were sampled in time intervals of 20 years in dynamic equilibrium state (150-500 years). Simple linear models and linear models including a quadratic term were fitted to the data and the minimal adequate model based on AIC values is shown here ( $\triangle \mathrm{AIC}>4)$.

The height-diameter relationship deviated slightly from the average allometry observed for South America by Banin et al. (2012) and overestimated the asymptotic maximum height by $\sim 3 \mathrm{~m}$ (Fig. 4.13 for details). The average vertical leaf area density profile of the simulated forest showed a unimodal distribution, in which the leaf area density peaked in the mid-canopy zone between 15 and $25 \mathrm{~m}$ (Fig. 4.13b). 

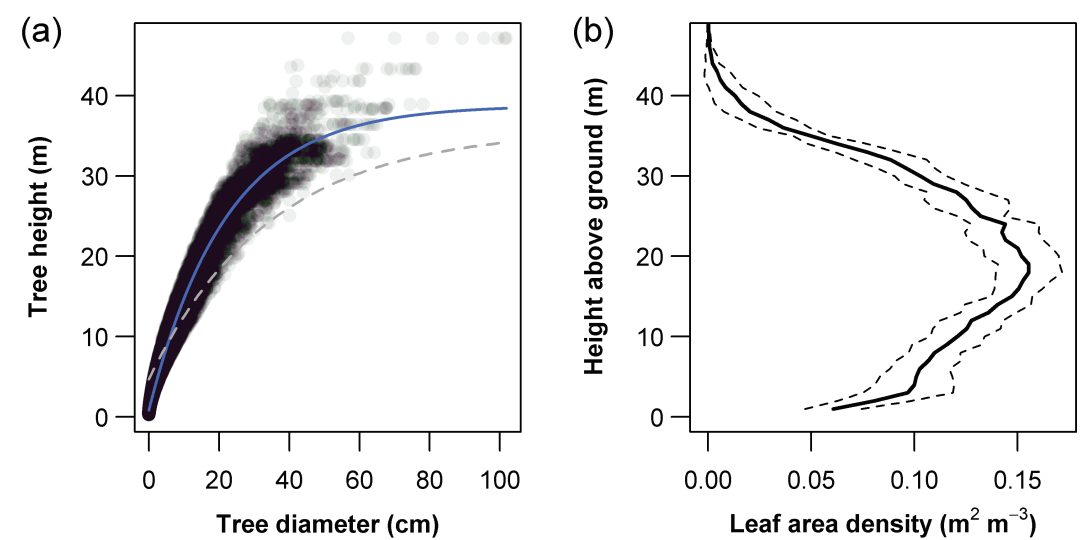

Figure 4.13. Height-diameter relationship (a) and vertical leaf area density distribution (b). (a) Each dot represents a single tree in the simulated forest stand. To reduce the degree of temporal pseudoreplication, all trees in the forest stand were sampled in time intervals of 20 years in dynamic equilibrium state (150-500 years). The relationship between tree height and diameter was described by the three-parameter exponential equation $L_{T}=H_{M a x}-a \cdot e^{\left(-b \cdot D_{T}\right)}$, where $L_{\mathrm{T}}$ and $D_{\mathrm{T}}$ are the height and diameter of the tree, and $H_{\mathrm{Max}}, a$ and $b$ are curve parameters ( $H_{\mathrm{Max}}$ represents the asymptotic maximum height, $a$ is the difference between maximum and minimum height, and $b$ the shape of the curve). This equation was used by Banin et al. (2012), who estimated an asymptotic maximum height of $H_{\mathrm{Max}}=35.8(a=31.1$, $b=0.029$ ) for Neotropical forests based on 49 forest plots (grey dotted line). Our model (blue line) predicted $H_{\text {Max }}=38.8$, $a=37.9$ and $b=0.045$. (b) The vertical leaf area density profile was calculated based on the simulated total leaf area in each voxel $A_{\text {LTot }}$. For each vertical $1 \mathrm{~m}$ layer, the mean $A_{\text {LTot }}$ was estimated. The sold black line shows the means over all time step in dynamic equilibrium state (years 150-500), and the dotted lines indicated the standard deviation.

Visualizations of the simulated forest stand (Fig. 4.14) illustrate the level of detail and structural realisms of the model.

(a)

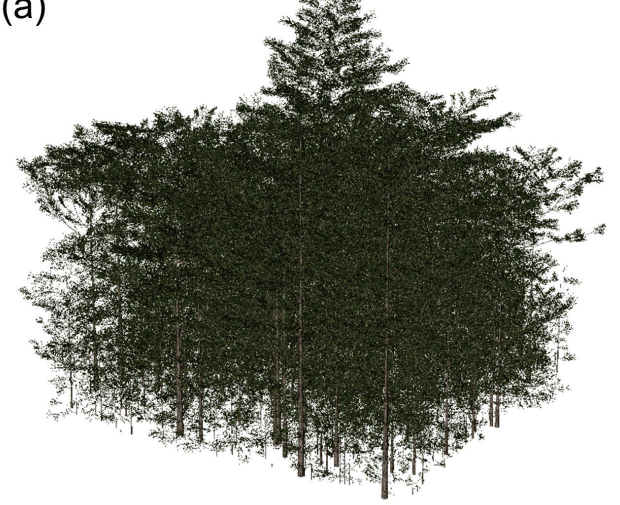

(b)

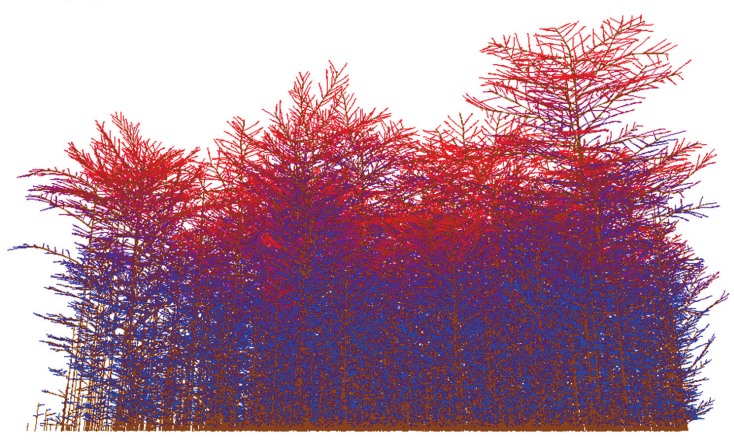

Figure 4.14. Visual representation of the simulated forest. (a) Oblique top view on simulated forest stand ( $0.25 \mathrm{ha})$ at a representative time step in in dynamic equilibrium state. The rendered representation is shown here (VisualizationShader $=1)$. (b) Side view on simulated forest stand $(0.25$ ha) at a representative time step in in dynamic equilibrium state. The wireframe representation is shown here, (VisualizationShader $=0$ ), where second order branches are colored according to the light conditions (VisualizationMethod=1). Colors represent the shift from high light intensities (red) to low light intensities (blue). 


\section{Functional-structural forest model}

Tree-level simulations revealed that tree growth under constant environmental conditions can be divided into three successive life stages (Fig. 4.15). The early life stage is characterized by a quick increase in height and diameter and continuously increasing net primary production rates. In this stage, all major branches are foliated (Fig. 4.15a). Subsequently, the lower branches begin to shed leaves (Fig. 4.15a), accompanied with lower increments in height and diameter and reduced net primary production (Fig. $4.15 \mathrm{~b}, \mathrm{~d}$ ). This stage ends when the tree reaches its maximum height. In the subsequent senescence stage, height and diameter growth cease and net primary production decreases as a result of the loss of photosynthetically-active leaf biomass. The leaf loss continues until all leaves are shed and the tree ultimately dies from carbon starvation.

(a)

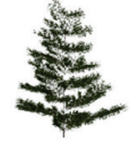

15 years

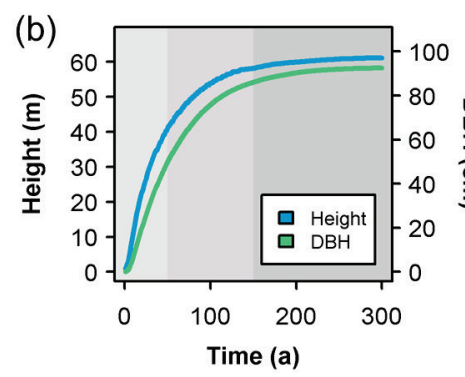

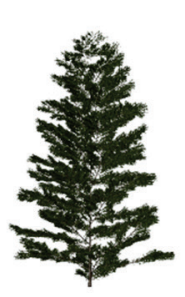

50 years

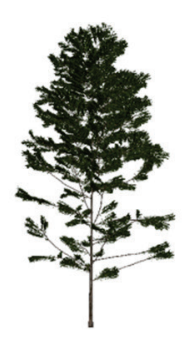

90 years

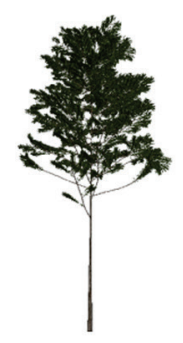

120 years

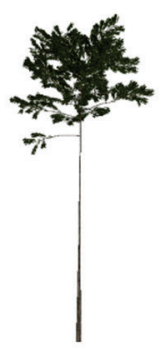

150 years
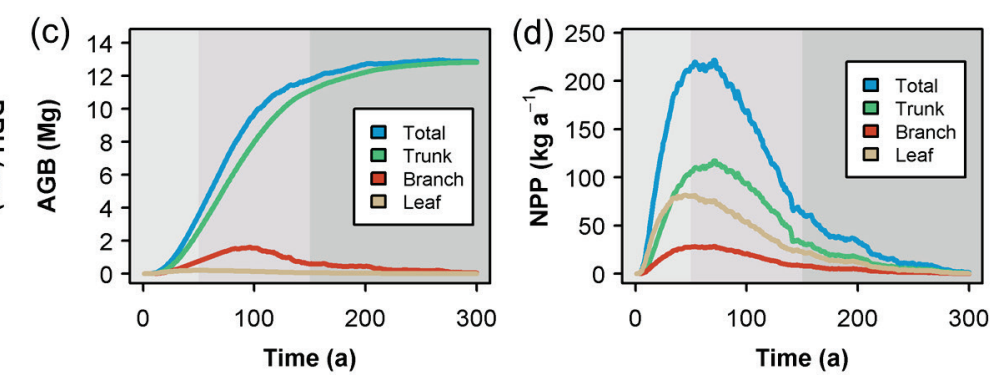

Figure 4.15. Ontogenetic development of an individual tree. (a) Visualization of tree structure of a freestanding tree at different ages. (b) Changes in tree height and diameter, (c) above-ground biomass (AGB) of the trunk, branches, leaves and the entire tree, and (d) net primary production (NPP) of the trunk, branches, leaves and the entire tree over time (panels b-d correspond to the tree shown in a). This example shows how a long-lived emergent tree species characterized by a low SLA growth without competition with neighbors over 300 years. Growth can be roughly divided into 3 life stages which are indicated by the different shades of gray in panels b- $d$. The first stage is characterized by a quick increase in height and diameter and continuously increasing net primary production rates. In this example, it ends at an age of $\sim 50$ years. In the subsequent stage the NPP decreases and the tree sheds lower branches. The final senescent stage begins at $\sim 150$ years when the tree growth close to its maximum height. In this stage it successively loses all leaves and branches, ending with the death of the tree.

All species generally followed the illustrated tree growth pattern over their life spans when grown under constant external conditions. However, tree traits and environmental conditions had a large influence on all aspects of growth (Fig. 4.16). Species with high SLA values showed high initial growth rates (Fig. 4.16c; bear in mind that the SLA values is representative for the position on the LES) and reached their maximum height rapidly. Consequently, they entered the senescence stage after a shorter time and died at a comparably young age (Fig. 4.16a-d). In contrast, species with low SLA values had a lower growth rate, but were able to maintain their growth rate for a 
longer time. They reached larger maximum heights at a higher age and had a longer life span. Wood density also affected life history growth, mainly by influencing the maximum height (Fig. 4.16e-h). Both external factors (light and site index) affected tree growth in a similar way (Fig. 4.16k-o). Due to the trade-off between carbon gain and carbon costs, lower light and site index values also decreased the maximum height. Interestingly, in contrast to SLA, variations in external factors clearly influenced the maximum height, but the maximum life-span was only slightly affected.
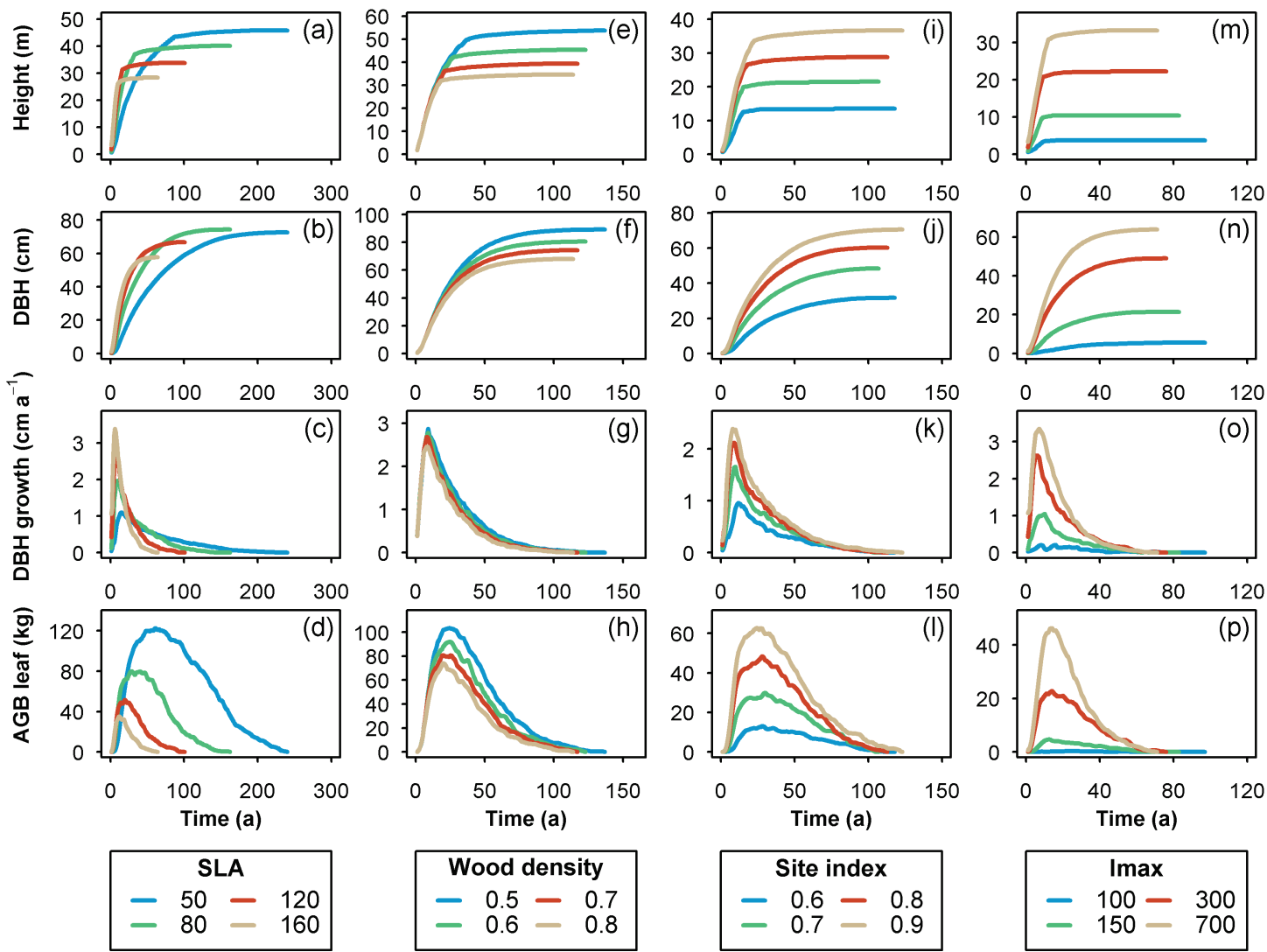

Figure 4.16. Tree dynamics in dependence of their traits and the environmental conditions. Development of height, diameter, diameter growth rates and leaf biomass of trees with otherwise identical functional and structural traits which only differ in their specific leaf area (SLA; a-d) or their wood density (e-h). The right-hand panels illustrate the effects of the site index (i-l) and the light intensity $I_{\max }(\mathrm{m}-\mathrm{p})$ on growth of trees with an identical set of traits.

An important aspect of this modelling approach is that the theoretical maximum height of tree species is an emergent property of their leaf traits. This is because the maintenance and construction costs for pipes connected to the leaves increase with increasing pipe length, and at a certain length these carbon cost exceed the amount of carbon assimilated by the leaves, i.e. the carbon budget is negative. This pipe length can be interpreted as maximum tree height, which increases with decreasing SLA (Fig. 4.17a). In a similar way, the maximum height is also 


\section{Functional-structural forest model}

correlated with wood density (Fig. 4.17a). In addition to the species traits, the maximum height also depends on the external conditions, i.e. light intensity and site index (Fig. 4.17b).

(a)

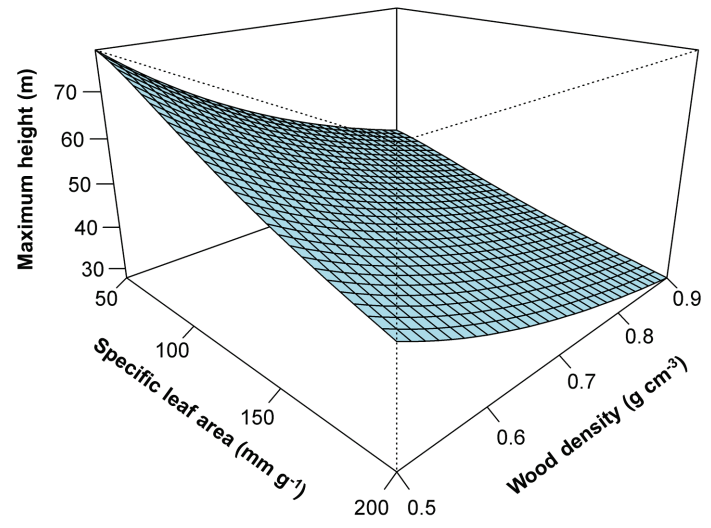

(b)

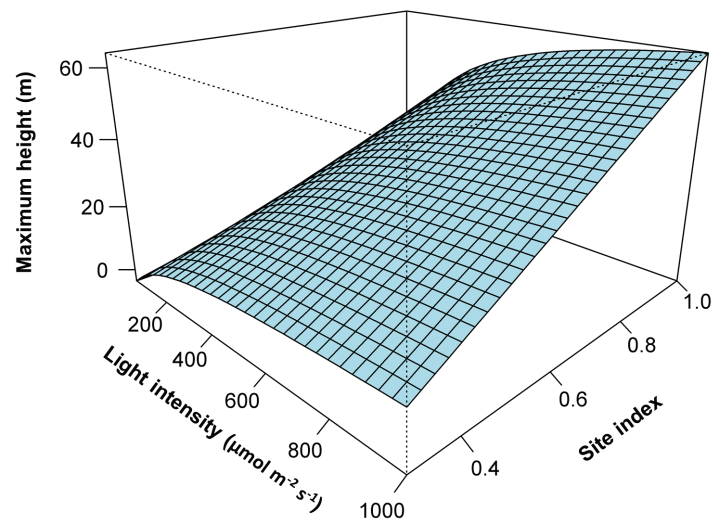

Figure 4.17. Maximum tree height as a function of (a) tree traits and (b) environmental conditions. The maximum height of a tree is directly related to the maximum pipe length $L_{\mathrm{PMax}}$, which is an emergent property of our model (4.41). The maximum height decreases with SLA and wood density (a), and increases with light intensity and site index (b).

Light conditions in forest stands are unevenly distributed, and trees can be subjected to a wide range of light intensities throughout their ontogeny. The light compensation point is thus an important characteristic to assess the light demand and to evaluate the survival probability of a species. In this model, the light compensation point can be assessed at the level of leaf compartments and mainly depends on the leaf investment strategy (expressed by the SLA) and the pipe length of the leaf compartment (Fig. 4.18). In the understory, i.e. with short pipe lengths, species with high SLA values have a lower compensation point than those with low SLA values. However, the compensation point of high SLA species steeply increases with increasing height, while the increase is shallower for low SLA species (Fig. 4.18). Consequently, the latter ones have lower light compensation points in the outer canopy zone. 


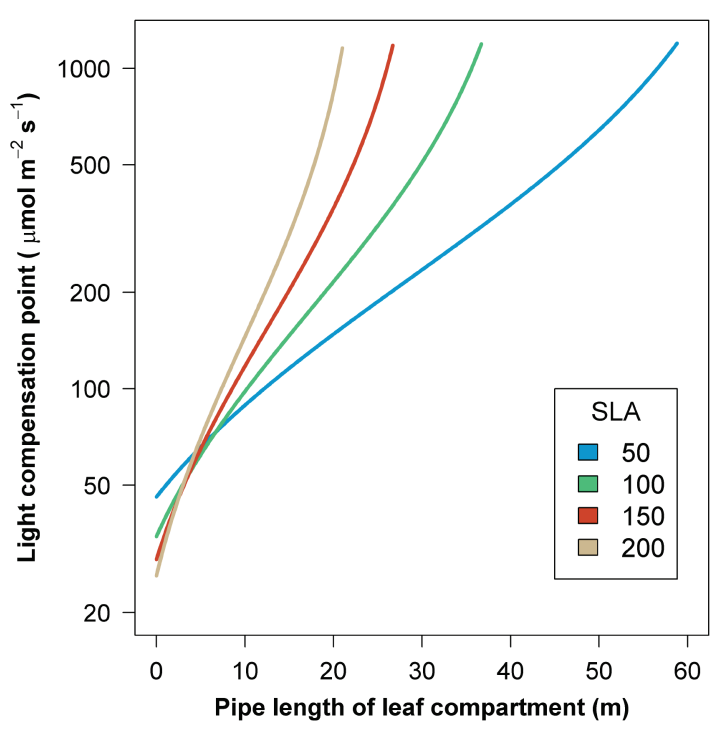

Figure 4.18. Light compensation point of a leaf compartment in dependence on SLA and the pipe length. Each leaf compartment of a tree forms a leaf-pipe element which acts as autonomous module. Consequently, the light compensation point, i.e. the light intensity at which the assimilation rate matches the respiration rate, can be assessed at this level.

\subsection{Discussion}

The main aim of our forest model was to simulate long-term tropical forest dynamics at the stand scale at a high level of structural detail. In comparison with other commonly used individual- or cohort-based forest models (e.g. Liu 1998; Köhler \& Huth 1998; Huth \& Ditzer 2000; Phillips et al. 2004a), there are two main differences. Firstly, each tree is simulated as a functional-structural tree model in which the 3D tree structure is represented in detail, including branch segments up to the second order and within-tree leaf distribution at $1 \mathrm{~m}^{3}$ resolution. This allows detailed simulations of crown competition and within-stand light regimes. Secondly, tree species are not a priori classified into distinct functional groups but drawn from the full trait space with continuously varying leaf and wood traits. As tropical forest are species rich (more than 300 tree species per hectare have been observed; Gentry 1988; ter Steege et al. 2013), functional groups aggregating tree species with similar growth characteristics are used in forest models for simplification, and parameters like growth rate or potential height are estimated for each group (e.g. Köhler \& Huth 1998; Tietjen \& Huth 2006). Here, as suggested by Wright et al. (2004), the leaf investment strategy of each species depends on the position on the continuous leaf economic spectrum, and scales up to the whole-tree level. It is important to note that growth, shade tolerance, and maximum height of each tree species emerge from the leaf investment strategy and are not defined a priori. To validate this model, we tested if ecological patterns at the forest level 


\section{Functional-structural forest model}

can be reproduced and if observed life-history variations among tree species can be simulated by differences in their leaf investment strategy.

\section{Forest level}

As first indication for structural realism of our model a dynamic equilibrium was reached, and in this equilibrium 12 important forest attributes fell within the ranges of Neotropical lowland forests (Fig. 4.10). It is generally assumed that under constant environmental conditions, carbon gains and losses are relatively balanced in old-growth forests, resulting in fluctuations around an equilibrium biomass level which are largely driven by gap dynamics (Whitmore 1990; Galbraith et al. 2014). The time required to reach biomass values typical for mature forests depends on environmental conditions. A wide range of time spans have been reported ranging from 40 to 60 years (Puerto Rico; Mitchell Aide et al. 2000; Marin-Spiotta, Ostertag \& Silver 2007), 75 years (Mexico; Hughes, Kauffman \& Jaramillo 1999) 100 years (Brazil; Fearnside \& Guimarães 1996) up to $\sim 190$ years (Columbia and Venezuela; Saldarriaga et al. 1988). Such time spans have not been observed directly but estimated using linear or non-linear models and are thus subject to uncertainty. Nevertheless, the simulated time span of 80 to 100 years lies well within this reported range.

Gap creation caused by falling trees is the main source of deviations from equilibrium, and single gap formation should be more significant at small stand sizes. The higher fluctuations at smaller plot sizes (Fig. 4.10) thus agree with expectations and are in line with simulations by Chambers et al. (2013).

Some attributes like stem density, basal area and above-ground biomass are well-studied, while other attributes describing forest dynamics (net primary production, turnover rates) and structure (leaf area index, canopy height) are less frequently measured. We considered the variability of all attributes, but also the higher uncertainty in the less frequently reported attributes, in estimating value ranges. Nevertheless, as we did not use statistical analyses, estimated ranges involved subjective decisions and thus should be interpreted with caution. However, the large number of attributes which were in reasonable ranges nevertheless indicates that this forest model produces structurally realistic results and captures essential mechanisms.

As an additional evaluation of model performance, we compared more complex patterns at the forest level. Crown width, crown area, and branching height are common measures of crown architecture, which, unsurprisingly, were positively correlated with tree height (Fig. 4.12). The linear increase in crown width with height is in accordance with linear or almost linear trends observed in many tropical tree species (King 1996; Alves \& Santos 2002; Iida et al. 2011). 
Interestingly, a bulge in the crown width-tree height relationship was observed for tall trees $>$ 30m (Fig. 4.12b), indicating a disproportionate increase in crown width when trees rise above the average canopy height. King (1996) made similar observations finding a much steeper slope in the crown width-tree height relationship for larger trees potentially growing above the canopy. Crown development of emergent trees is less constrained by competition for space, and our model is able to reproduce such plastic crown responses. With an average crown width of $10 \mathrm{~m}$ at a height of $20 \mathrm{~m}$, our model slightly overestimates crown dimension compared to observation, which rather were around 7-9 m (King 1996; Alves \& Santos 2002). On the one hand, differences in methods to estimate crown width might partly explain the deviation. The exact position of each branch segment is known in the model and the crown width thus represents the absolute maximum distance to the stem, which might lead to a slight overestimation compared to ground-based field observations. On the other hand, due to the relatively simple integration of space competition in this model, the area of overlap between two crowns might be larger than in nature, leading to wider crowns. Crown area is generally closely related to crown width and the non-linear increase agrees with observations (Alves \& Santos 2002; Poorter, Bongers \& Bongers 2006). While the average trend might also be slightly higher than expected, the simulated range is in line with the results of Poorter et al. (2006), who found large differences in crown area trends for 54 tropical tree species.

Compared with the discussed crown measures, branching height was less strongly correlated with tree height (Fig. 4.12a). ). In our model, branches are generated following the architecture defined by species structural traits, and branch shedding results from physiological process, i.e. branches are shed when all leaves are lost. These processes lead to a distinct development of branching height during tree ontogeny (Fig. 4.15), which can be modified when trees compete for space in forest stands (Fig. C.1). In natural trees, branching architecture is a complex trait, and the processes of branch generation and branch shedding are likely related to within-tree optimization of carbon gain (Farnsworth \& Niklas 1995). For example, the complex crowns of emergent trees often develops trough reiteration from dormant buds, and this process is influenced by the local light conditions (Hallé et al. 1978; Barthélémy \& Caraglio 2007). Due to such additional optimization mechanisms, which are not implemented in this model, the correlation between branching height and tree height is probably stronger in natural forests. However, direct comparisons are difficult because such correlations have only been studied at the species level (Alves \& Santos 2002; Iida et al. 2011) and analyses at the forest plot level are not available. Nevertheless, the average trend in our study largely agrees with the average trends in different tree species (Alves \& Santos 2002; Iida et al. 2011).

The simulated leaf area density peaked in the canopy zone around $20 \mathrm{~m}$ (Fig. 4.13b), which is in accordance with observations in lowland forests on Barro Colorado Island (Taubert et al. 2015) 


\section{Functional-structural forest model}

or near Manaus (Stark et al. 2012). In the latter study, however, an additional increase in leaf area density near the forest floor was observed, probably due to herbaceous vegetation which is not included in our model. Although vertical leaf area profiles have not been as extensively studied as other forest attributes, a leaf area maximum in the canopy layer is generally expected for undisturbed old-growth forests and our simulations are in line with this expectation. Interestingly, in a lowland forest analyzed by Stark et al. (2012), the leaf area density peaked in the lower canopy around $10 \mathrm{~m}$. These authors considered past disturbances and the resulting nonequilibrium forest state as a possible cause of the deviating pattern. Analyzing the effect of disturbance regimes on the vertical leaf area distribution might thus be an interesting future application of this model.

The asymptotic height of the diameter-height relationship observed here ( $38.8 \mathrm{~m}$; Fig. 4.13a) is close to the observed mean for Neotropical forests (35.8 $\pm 6.0 \mathrm{~m}$; Banin et al. 2012). However, the shape of the simulated relationship differed from the observed average trend, and the simulated height at smaller diameter classes was slightly overestimated (Fig. 4.13a). In our model, height scales with diameter to the power $2 / 3$, controlled by species-specific shape parameters. Trees only deviate from their species-specific relationship under low light conditions (increased height growth) or at maximum height (cessation of height growth). A more advanced allometric scaling model might thus improve our model. For instance, it is known that trees show a plastic response to several environmental conditions (light, precipitation, stand density) that can alter the intraspecific allometric relationships (Feldpausch et al. 2011; Banin et al. 2012). Trees might, for instance, cease height growth when growing as emergents and rather extend their crown horizontally.

Our model produced a right-skewed tree diameter distribution (Fig. 4.11a), which is consistent with observations from other tropical forest (Oliveira-Filho et al. 1994; Hector et al. 2011). In addition, the simulated normal or slightly skewed distributions for height and age of trees $>10$ cm in DBH (Fig. 4.11b,c) agrees with empirical studies (Campbell et al. 1986; Oliveira-Filho et al. 1994; Worbes et al. 2003; Adekunle, Olagoke \& Akindele 2013). As diameter, height, and age are usually correlated, similar frequency distribution of these attributes can be expected when considering all trees. Interestingly, the height distribution deviated from the age distribution and showed a distinct hump between 25 and $35 \mathrm{~m}$ (Fig. 4.11b). We speculate that the crowns of these trees in the upper canopy are well illuminated and less exposed to between-crown competition for space. Consequently, the probability to die due to carbon starvation should be lower, which might explain why the negative trend is halted for these larger height classes.

The metabolic theory of ecology predicts a linear decrease in stem diameter frequency on a loglog scale. Muller-Landau et al. (2006) and Enquist, West \& Brown (2009) confronted the 
theoretical predictions with observations and found deviations particularly among the larger diameter classes, whose frequency was lower than predicted. Interestingly, our model shows similar deviations (Fig. 4.11d). Enquist, West \& Brown (2009) speculated that other sources of mortality than competitive thinning are the reason for the predicted linear trend, for instance wind damages, herbivory or diseases, which are particularly severe in larger size classes. In our model, trees growing at their maximum height enter senescence which inevitably ends with death. This emergent model behavior thus reflects the increased mortality probability of very large trees, leading to model results which are close to observations.

In summary, our model reproduce a variety of patterns at the forest level reasonably well. Despite the overall complexity of the model, it is based on relatively simple assumptions: A local species pool is generated by randomly selecting species traits, these species germinate at random positions within the model space, they compete for light and space, and their mortality rates generally decrease with size and light conditions. The forest dynamics emerges from these lower level processes, and the agreement between multiple forest patterns and the model results is thus an indication for the suitability of our model approach.

\section{Tree level}

As an additional test of model performance, we analyzed simulated growth patterns of individual trees (Fig. 4.16). We hypothesized that differences in leaf traits capture observed life-history variations among different tree species. Tree species are often divided into functional groups (Gourlet-Fleury et al. 2005; Chazdon et al. 2010) and the simplest concept distinguishes pioneer and shade-tolerant species (Swaine \& Whitmore 1988). Pioneer species are generally fastgrowing, short-lived species not able to establish under shade. Shade tolerant or climax species, in contrast, can survive under low-light conditions in the understory; they grow slower but live longer, potentially enabling them to grow as emergent trees. While a classification into functional groups can often be useful, several studies indicate that the transition from fast growing, shortlived pioneer to slow growing, long-lived shade-tolerant species is indeed rather continuous (Wright et al. 2003b; Poorter \& Bongers 2006). In our model, variations in leaf trait, expressed by the SLA, resulted in such variations in life history patterns (Fig. 4.16a-d). As pioneers generally have a significantly higher SLA than shade-tolerant species (Kitajima 1994; Rijkers et al. 2000), our result are consistent with these observations (Wright et al. 2003b; Poorter \& Bongers 2006).

The leaf trait trade-offs integrated in this model (Wright et al. 2004) are linked to whole-tree performance and support the notion that the growth-survival trade-off of tropical tree species is, 


\section{Functional-structural forest model}

at least partly, determined by their leaf traits (Sterck et al. 2006). With regard to their light requirements, it is, at first glance, rather counterintuitive that pioneer species with their high SLAs are unable to survive in the shade. A high SLA means a high photosynthetically-active leaf area per dry mass investment (Evans \& Poorter 2001), which should be favorable under low light availability. Interestingly, under controlled conditions in an experimental setup, the growth rates of pioneer seedlings in shade were actually higher than those of shade-tolerent species (Kitajima 1994). Futhermore, leaf traits within individual trees are usually not invariable but rather show a plastic response to shade (this issue is dicussed below in more detail), with shade leaves having higher SLA values than sun leaves (Rozendaal et al. 2006; Markesteijn et al. 2007). These examples indicate an increased efficiency of light capture with increased SLA. However, this comes at a cost: leaves with a high SLA are short-lived, and low leaf toughness makes them more susceptible to herbivory and physical damage (Coley 1983; Wright \& Cannon 2001; Díaz et al. 2004). The carbon loss associated with these negative effects of a high SLA seem to be particularly important in the understory and increases the mortality rate of such species (Kitajima 1994). Consequently, a conservative ressource use associated with a low SLA (tougher leaves, higher longevity) thus generally favors survival in shade and explains why shade-tolerant species are characterised by low SLA. Interestingly, our model simulates a similar light-dependent growth which is related to resource use efficiency. When trees are small, a higher SLA is more efficient, resulting in a lower light compensation point (Fig. 4.18). However, the increased leaf turnover of high SLAs has an adverse effect with increasing tree height, resulting in higher leaf compensation points above a certain height compared to low SLA species. Consequently, under low light conditions species with low SLAs are able to maintain a positve carbon buget over a wider range of tree heights, favoring their survival in the lower canopy (Fig. 4.18).

Differences in wood density influenced tree growth much less than leaf trait differences (Fig. 4.16). The most obvious effect was an increase in maximum height (and diameter) with decreasing wood density. Observed relationship between maximum height and wood density are not consistent across studies, and while the trend observed for Iberian canopy tree species agrees with our simulation results (Poorter et al. 2012), other studies found no significant relationships (Wright et al. 2010) or even positive trends (Osunkoya et al. 2007). Our model is carbon-based and by decreasing the construction and maintenances costs per volume of wood, low wood densities are advantageous and allow trees to grow taller. However, low wood densities are usually associated with lower mechanical stability and increased vulnerability to hydraulic failures (Hacke et al. 2001; Anten \& Schieving 2010). Such trade-offs among wood traits influence the tree architecture and might have adverse effects on the maximum attainable height (van Gelder, Poorter \& Sterck 2006). Furthermore, correlations among wood traits are also regarded as a factor explaining why increasing mortality rates with decreasing wood density are 
consistently reported (Chao et al. 2008; Wright et al. 2010; Visser et al. 2016) - a pattern we did not observe in our simulations (Fig. 4.16). Thus, considering only wood density in our carbonbased model is not sufficient to reproduce all interspecific differences related to wood traits. Our model could be improved by integrating additional mechanisms in future studies, for instance by considering the mechanical stability or hydraulic properties of stems and branches, or by considering several wood traits and their correlations. However, in this study we focused on leaf traits rather than on wood traits.

The site quality, characterized by the dimensionless site index, was positively correlated with tree growth rates and maximum tree heights (Fig. 4.16i-1). We are not explicitly simulating water/nutrient cycles or temperature dependencies in our model, and the site index thus represents the strength of factors limiting tree growth, such as low water availabilities, low temperatures or poor soil conditions. Such limiting factors are generally associated with decreasing productivity and lower tree/canopy heights (Girardin et al. 2010, 2013; Pan et al. 2013), and the simulated patterns are thus qualitatively in accordance with these observations. Changing light conditions had similar effects on tree growth pattern, and the simulated increasing growth rates with light intensity are consistent with observed light-dependent responses of most tropical species (Kobe 1999; Rüger et al. 2011; Philipson et al. 2014).

Irrespective of environmental conditions and functional traits, simulated growth was clearly sizedependent and trees showed characteristic ontogenetic growth trajectories (Fig. 4.15-4.16). A significant effect of size on growth rates has also been observed for most tree species in field studies (e.g. Rüger et al. 2011; Iida et al. 2014). However, both increasing and decreasing growth rates with diameter (Rüger et al. 2012), as well as humped-shape responses are reported (Clark \& Clark 1999; Davies 2001). These observed differences might be related to species-specific variations, or incomplete or unbalanced data sets; tree growth data from natural forests often do not cover the entire size ranges of the species or are skewed towards the more frequent smaller size classes. It is thus not straightforward to estimate solid ontogenetic growth patterns based on field data, and whole-life growth trajectories are thus still debated (Rüger et al. 2011; Bowman et al. 2013). However, Hérault et al. (2011), found that the growth trajectories of 50 rain forest species could well be predicted using hump-shaped size-dependent models, and several additional empirical and theoretical studies suggest similar trajectories (e.g. Clark \& Clark 1999). Our simulations generally agree with such studies suggesting reduced growth rates at larger size, although the transition from increasing to decreasing growth rates is likely much smoother than in our model. In reality, trees exhibit a variety of mechanisms to optimize their carbon budget, and integrating such mechanisms could substantially improve the simulated tree growth trajectories (see limitations section below). Such detailed optimization mechanisms were, however, beyond the scope of this study. 


\section{Functional-structural forest model}

In summary, our model was able to reproduce ontogenetic 3D growth pattern of trees under different environmental conditions quite well. The tree's leaf traits had a particularly strong impacts on life history patterns and leaf trait variations reproduced important differences in ontogenetic growth trajectories and light requirements among functional tree types, ranging from fast-growing pioneers to shade-tolerant emergent species. Our models considers correlations among leaf traits according to the LES and the simulations results thus corroborate our hypothesis that life-history variations among tree species are largely driven by leaf trait trade-offs.

\section{Model limitations}

Model parameterization can be hampered by the relatively large number of free model parameters and the considerable computation time accompanying complex models such as ours. Recently proposed methods for model parameterization, such as Bayesian approaches for parameterization (e.g. Martínez et al. 2011; Matsushita et al. 2015), are data- and computationally-intensive and were thus not feasible in this study. Still, we were able to calibrate our model manually following pattern-oriented modelling (Grimm et al. 2005; Grimm \& Railsback 2011). Using this method, however, parameter uncertainty or collinearities among parameters could not be explored in detail. Nevertheless, we performed a simple sensitivity analysis in which the effects of changes of important model parameters on the model results were recorded (Table C.7), which might be useful for further model applications.

Data availability was an additional limitation. While long-term inventory data spanning years to decades for tropical forest are available for some plots (e.g. Condit 1995; Bradford et al. 2014), these inventories concentrate on few key forest attributes such as the stem number or the basal area. To our knowledge, long-term records for such a wide range of attributes as used in this study (e.g. LAI, canopy NPP, etc.) for the same forest plots are not available. Thus, we decided to use estimated ranges of multiple forest attributes in typical Neotropical instead of time-series data for a lower number of attributes from a specific location to validate our model. We believe that the chosen approach is appropriate for the purpose of this study; however, fitting a model to timeseries data would be highly informative because deficiencies in the model structure would become more obvious if multiple forest attributes could not be simultaneously reproduced.

Apart from the mentioned general limitations in model parameterization and validation, some ecological patterns could not be perfectly reproduced by our model. For instance, in natural forests trees commonly show fast initial height growth rates, but when crowns are well-illuminated, they tend to cease height growth and continue to grow in diameter at high rates (e.g. Matsushita et al. 2015). In this model, height and diameter growth tends to be coupled more tightly, and trees 
showed decreasing diameter growth rates after they entered senescence. While such decreases at large diameters were sometimes also found in nature (Clark \& Clark, 1999), the time frame over which large diameter growth rate could be maintained appear to be larger than in our simulation. This deviation might be explained to a large extent by the exclusion of intra-individual trait plasticity in our model. Each individual in this model is characterized by non-variable traits, while in nature plasticity in functional and structural traits is ubiquitous. For instance, adjustments of SLA in response to the light conditions are usually observed (Rozendaal et al. 2006; Markesteijn et al. 2007), and our model provides an adequate explanation why such adjustments are favorable (see Fig. 4.18). Futhermore, tree architecture can also be adjusted and trees tend to arrange their branches in such a way as to avoid self-shading and maximise carbon gain. In addition, reiteration from dormant buds is regarded as important additional branching mechanism in the canopy zone (Hallé et al. 1978; Sterck \& Bongers 2001; Osada 2011). If such plastic responses were integrated, larger trees would likely be able to use the available ressources more efficienly and they could maintain higher growth rates over a longer period. This would also mean that they could reach larger diameters and ages. In this model, maximum diameters of $\sim 100 \mathrm{~cm}$ and ages $\sim 250$ years were simulated, however, in real tropical forests, trees exceeding these limits are not uncommon. Despite the mentioned deviations, our model concentrating on between-species trait differences already reproduced many patterns. In the future, trait plasticity could be implemented in this model to further increase the number of adequately reproduced structural patterns.

\section{Conclusion and outlook}

This modelling study indicates a strong reationship between a tree's leaf traits and life history traits. We found that the position on the LES, which defines a specific set of economic leaf traits, determined the maximum height and age of a tree, as well as it size-dependent growth rates and shade tolerance. The simulated transition from fast-growing short-lived pioneers to slow-growing long-lived emergent species along the LES was consitent with our expectation of functional tree types, and we regard these consistencies as clear indication for a fundamental role of leaf traits in determining the life history growth patterns of trees. Moreover, when integrated into a forest stand model, many forest level patterns emerging from lower-level processes could sucessfully be reproduced, which is an additional indication for the usefulness of our model approach.

Bottom-up functional-structural tree and forest models have the potential to considerably increase our understanding of the mechanisms controlling tree and forest dynamics in the future. Due to the large and complex structures of trees and their long life spans, empirical studies on 3D tree growth are challenging. We are able to measure processes at lower organizational levels (e.g. photosynthesis at the leaf scale) or to track some tree variables over a limited time (e.g. diamter 


\section{Functional-structural forest model}

growth of saplings), but it is virtually impossible to record 3D tree growth as a whole. In this situation, functional-structural tree models can be helpful tools accompanying empirical studies, because they allow to test and evaluate the consequences of low-level mechanisms on whole-tree growth patterns, which, in turn, can help to obtain a more complete picture of whole-tree growth. So far, FSTM have not received much attention and thus offer ample opportunities in future studies. For instance, the importance of within-individual trait plasticity on whole-tree carbon budget could be explore, or a water budget model could be integrated and the effects of wood and leaf hydraulic traits could be assessed. Such an approach can be useful to test hypotheses on the role of hydraulic failure and carbon starvation in tree mortality. A better mechanistic understanding of tree growth and mortality based on FSTMs can also facilitate the development of next-generation predicitve forest models in which tree performance emerges exclusively from functional traits. Futhermore, we strongly recomment to consider the forest level as additional hierachical level in functional-structural modelling studies. On the one hand, bottom-up mechanisms can be tested under more realistic conditions, in which trees and envrionmental conditions interact in a complex way. On the other hand, such detailed forest models have the potential to advance our understanding of forest dynamics, specifically over long time frames. For example, the long-term effect of frequent disturbances or varying environmental conditions on forest stability (dynamic equilibrium) and structure (e.g. the vertical leaf area distribution) could be assessed. Last but not least, we want to highlight the potential of FSFM for future modelbased studies of canopy-dwelling organisms. Tropical forest canopies harbor numerous arboreal animals and epiphytic plants, but due to logistical problems accessing their habitat studies are often cumbersome. FSFM provide detail information on the 3D forest dynamics (e.g. tree and branch dynamics) and microclimatic changes, and this data can form the basis for further theoretical studies. For instance, our knowledge on long-term dynamics of vascular epiphytes is still very scarce, and by coupling a FSFM with an epiphyte population model, the importance of (changes in) 3D forest dynamics on epiphyte communities can be tested. Such analyses are particularly timely as tropical forests are already changing in response to atmospheric changes, and more information on the response of canopy-dwelling organisms to such changes is urgently needed. 
5 Simulating the influence of forest dynamics on structure and dynamics of epiphyte assemblages based on a coupled forest-epiphyte model

Gunnar Petter, Gerhard Zotz, Holger Kreft, Juliano Sarmento Cabral 



\subsection{Abstract}

Forest dynamics undoubtedly influence the structure and dynamics of epiphyte assemblages, for example by varying local microhabitat conditions and the branch surface over time. However, quantitative field studies in the three-dimensional habitat of epiphytes are generally tedious and costly. Unsurprisingly, our knowledge on structure and dynamics of epiphyte assemblages is thus scarce. In this study, we present a complementary approach to epiphyte research by developing the first mechanistic model conceived to simulate vascular epiphyte dynamics. The model was designed to be coupled with detailed forest models providing the habitat dynamics. By first applying the model to average dynamics of Neotropical lowland forests, we validated the spatial distribution of epiphytes. Thereafter, we assessed how differences in natural forest dynamics, selective logging of large trees and the size of forest stands influence the long-term dynamics of epiphyte assemblages. To this end, we generated reasonable forest scenarios using a dynamics functional-structural forest model, and simulated the epiphyte dynamics on these forest stands over 500 years. Forests with low natural tree turnover rates had lower epiphyte extinction rates, higher abundances and were more 'saturated'. Even in mature lowland forest, epiphyte assemblages commonly show no sign of saturation, and our simulations demonstrated that the saturation level was strongly influenced by the dynamics of the forest. Furthermore, an increased logging intensity or a decreased size of the forest stand had negative impacts on the epiphyte community and resulted in higher local extinction rates. Our results demonstrated that the average abundance and biomass of epiphytes are regulated by forest dynamics. Such influences of forest dynamics on epiphyte assemblages should thus be considered in epiphyte research in addition to the known influences of environmental factors such as water-availability. We conclude that mechanistic models can be valuable tools to increase our understanding of the dynamics of epiphyte communities and to provide useful feedbacks to both empirical studies and conservation policies.

\subsection{Introduction}

Vascular epiphytes are a taxonomically diverse group comprising $\sim 9 \%$ of the world's plant species (Zotz 2013). These non-parasitic plants germinate and grow on other plants, usually trees, without contact to the soil. Their arboreal life style allows epiphytes to reach well-illuminated zones in forest canopies without major investments in plant structure (Benzing 1990). Due to the often low plant densities, competition for space and light seems to be of minor importance in most epiphyte assemblages, which may be an additional advantage over terrestrial plants (Zotz \& Hietz 2001). However, as epiphytes are isolated from terrestrial soils, they have to cope with low and 


\section{Forest-epiphyte model}

irregular supply of water and nutrients from atmospheric inputs, litter or canopy soils (Benzing 1990; Wania et al. 2002). To enhance resource uptake and retention, epiphytes have evolved a number of adaptations such as water-storing phytotelmata, pseudobulbs or the velamen radicum facilitating their survival in forest canopies (Benzing 1990).

The explicit three-dimensional nature of the epiphyte habitat implies a strong dependence on forest structure and dynamics. The forest structure, i.e. the spatial distribution of trunks and branches, influences demographic processes of epiphytes directly by providing colonizable substrate, but also indirectly by controlling the spatial distribution of abiotic factors (Parker 1995). These direct and indirect effects are the main causes of the spatial structure of epiphyte assemblages within forests and within individual trees (Johansson 1974; Zotz 2007). Within forests, the typically observed vertical stratification of epiphyte species is commonly attributed to the pronounced vertical gradients in light and humidity (ter Steege \& Cornelissen 1989; Benzing 1990). For instance, for species growing in the upper canopy, efficient transpiration control and leaf succulence is an advantage, while in the dark understory efficient light use via high specific leaf areas (SLA; the ratio between photosynthetically-active leaf area and dry mass) facilitates survival (Petter et al. 2016).

Also within individual trees, epiphyte species are not evenly distributed, and Johansson (1974) proposed a zonation scheme based on the relative within-tree position, which is still widely used in epiphyte ecology. In addition to microclimatic gradients from the outer to the inner crowns similar to those at the forest level, the importance of the spatial distribution and temporal dynamics of substrate for epiphyte colonization and mortality becomes particularly evident at the tree level. Trees are dynamic systems in which new substrate is continuously generated by growth processes, but also lost via branch fall. As the frequency of branch fall is size-dependent (Cabral et al. 2015), the age and longevity of the substrate also shows pronounced within-tree gradients from stable trunks to highly dynamic outer crowns, and this gradient contributes to the spatial pattern of epiphytes within trees.

Population and community dynamics of epiphyte assemblages are influenced by the outlined complex forest-epiphyte interactions at different spatial and temporal scales. As differences in climatic conditions influence both epiphytes and forests (Quesada et al. 2012), an indirect climate effect on epiphyte assemblages via variations in forest structure and dynamics can be assumed. So far, epiphyte studies mainly focused on direct effects of climate on epiphyte diversity, and water-related variables such as precipitation and humidity are considered as important determinants (Gentry \& Dodson 1987; Kreft et al. 2004). However, a recent study by Ding et al. (2016) disentangled the direct effects of climate and soil on epiphyte diversity and abundance along an elevational gradient from the indirect effects via forest structure using structural equation 
models. Interestingly, indirect effects explained a similar proportion of variations in abundance and species richness as humidity, which highlights the fundamental impact of the forest structure on epiphyte assemblages.

Sampling and monitoring epiphyte in their three-dimensional habitat is logistically challenging as it requires climbing or technical equipment like canopy cranes, airships or canopy platforms. Consequently, studies on composition, structure and particularly dynamics of epiphyte populations and assemblages are rare compared to the numerous floristic works on epiphytes. Mondragón, Valverde \& Hernández-Apolinar (2015) recently reviewed studies about the population ecology of epiphytic angiosperms and found population growth rates based on matrix analyses for only 30 epiphyte species of 2 families (bromeliads, orchids) - a very low number compared to the total diversity of epiphytes ( $>27.000$ species and 73 families, respectively; Zotz 2013). Regarding temporal dynamics of entire epiphyte assemblages, two repeated plot-scale censuses (1 ha plot in Venezuela: Schmit-Neuerburg 2002; 0.4 ha plot in Panama: first census by Zotz \& Schultz 2008, second census by G. Mendieta-Leiva, K. Wagner \& G. Zotz, unpublished data) and two studies assessing temporal changes on specific host tree species (Socratea exorrhiza: Laube \& Zotz 2006; Annona glabra: Zotz, Bermejo \& Dietz 1999) have been conducted so far. Compared to soil-rooted plants data availability is thus limited, and in combination with the lack of standardized methodology and terminology in epiphyte studies (Mendieta-Leiva \& Zotz 2015), this makes it difficult to develop a general theory on structure and dynamics of epiphyte assemblages.

Mechanistic models are valuable tools to increase theoretical knowledge in ecology, which in turn can inform field studies. Mechanistic models can help to disentangle the complex interactions between forests and epiphytes by simulating and evaluating the effects of different hypothesized mechanisms. In this study, we analyzed the effects of long-term forest dynamics on the structure and dynamics of epiphyte assemblages by coupling a functional-structural forest model (FSFM) with an individual-based epiphyte model. The FSFM simulates structural growth, establishment and mortality of virtual three-dimensional trees at the stand scale, and each tree is represented by a trunk and numerous branch segments up to the $2^{\text {nd }}$ order. Tree growth includes growth of trunks and branches as well as establishment of new branches and branch fall. By coupling the FSFM with an epiphyte model, detailed processes such as the fall of epiphyte attached to branches can be simulated. We modelled different forest scenarios, which allow us to assess how (1) differences in natural forest dynamics, (2) selective logging and (3) the size of the forest fragment influence the long-term dynamics of epiphyte assemblages. 


\section{Forest-epiphyte model}

\subsection{Materials and methods}

\subsubsection{Model description}

The model description follows the ODD (Overview, Design concepts, Details) protocol which was proposed as standard protocol to communicate agent-based models (Grimm et al. 2006, 2010).

\subsubsection{Purpose}

The main purpose of this model is to analyze the influence of long-term forest dynamics on the structure and dynamics of vascular epiphyte assemblages. Vascular epiphytes germinate and grow on trees. Thus, the fate of individuals is connected with the dynamics of their host trees, which grow and create new substrate, but which also shed branches and ultimately die and fall (Sarmento Cabral et al. 2015; Taylor \& Burns 2015). Driven by natural environmental differences or by human interventions, the dynamics of different forests can differ substantially (Brown et al. 2004; Wright 2005; Quesada et al. 2012). In this study, the impact of such differences on epiphyte assemblages is studied by coupling a detailed three-dimensional forest model with an individualbased epiphyte model.

\subsubsection{Entities, state variables and scales}

The model is three-dimensional and voxel-based, and its spatial extent depends on the spatial dimensions of the coupled forest model, which usually covers an area of 0.25 to 1 hectare at a height of ca. $50 \mathrm{~m}$. The model space is subdivided into voxels of $1 \mathrm{~m}^{3}$, whose state variables characterize three key environmental conditions: i) light intensity, ii) total area of arboreal substrate, and iii) relative loss of substrate area (Table 5.1). The model proceeds in annual time steps, commonly covering a period of 500-1000 year. The state variables of the voxels are updated each year according to the result of the forest model (Fig. 5.1). Individual epiphytes are the actual entities whose growth, reproduction and mortality are simulated as a function of their traits and the environmental conditions in the voxels. The state variables and traits of epiphytes are summarized in Table 5.1. 
Table 5.1. State variables and species-specific traits. The demographic processes of individual epiphytes in this model are influenced by the state variables of the voxels describing the environmental conditions, and by the specific traits of each species to which an individual epiphyte belongs to.

\begin{tabular}{|c|c|c|c|}
\hline Symbol & Description & Unit & Type \\
\hline$V_{\mathrm{x}}$ & Position of voxel in model space in $\mathrm{X}$ direction & $\mathrm{m}$ & State variable (voxel) \\
\hline$V_{Y}$ & Position of voxel in model space in $\mathrm{Y}$ direction & $\mathrm{m}$ & State variable (voxel) \\
\hline$V_{z}$ & Position of voxel in model space in Z direction & $\mathrm{m}$ & State variable (voxel) \\
\hline I & Light intensity & $\mu \mathrm{mol} \mathrm{m} \mathrm{m}^{-2} \mathrm{~s}^{-1}$ & State variable (voxel) \\
\hline $\mathrm{S}_{B}$ & Total surface area of arboreal substrate & $\mathrm{m}^{2}$ & State variable (voxel) \\
\hline$S_{\text {Loss }}$ & Percentage annual surface loss & $\%$ & State variable (voxel) \\
\hline$I D_{\text {Ind }}$ & Individual ID of epiphyte & - & State variable (epiphyte) \\
\hline$I D_{\mathrm{sp}}$ & Species ID of epiphyte & - & State variable (epiphyte) \\
\hline Ex & Position of epiphyte in model space in $\mathrm{X}$ direction & $\mathrm{m}$ & State variable (epiphyte) \\
\hline EY & Position of epiphyte in model space in Y direction & $\mathrm{m}$ & State variable (epiphyte) \\
\hline$E_{z}$ & Position of epiphyte in model space in Z direction & $\mathrm{m}$ & State variable (epiphyte) \\
\hline M & Mass of epiphyte & g & State variable (epiphyte) \\
\hline$A$ & Age of epiphyte & years & State variable (epiphyte) \\
\hline$M_{\text {Max }}$ & Maximum mass & g & Species-specific trait \\
\hline$M_{\text {Mat }}$ & Mass at maturity & g & Species-specific trait \\
\hline$K$ & Growth rate (Bertalanffy growth) & $a^{-1}$ & Species-specific trait \\
\hline$D$ & Dispersal ability (negative exponential dispersal) & - & Species-specific trait \\
\hline$R$ & Maximum number of recruits per individual & \# & Species-specific trait \\
\hline$I_{\text {Min }}$ & Minimum light intensity for survival & $\mu \mathrm{mol} \mathrm{m} \mathrm{m}^{-2} \mathrm{~s}^{-1}$ & Species-specific trait \\
\hline$I_{\operatorname{Max}}$ & Maximum light intensity for survival & $\mu \mathrm{mol} \mathrm{m} \mathrm{m}^{-2} \mathrm{~s}^{-1}$ & Species-specific trait \\
\hline$I_{\text {Opt }}$ & Optimum light intensity & $\mu \mathrm{mol} \mathrm{m} \mathrm{m}^{-2} \mathrm{~s}^{-1}$ & Species-specific trait \\
\hline$I_{\mathrm{A}}$ & Parameter A of parabolic light response curve & - & Species-specific trait \\
\hline$I_{\mathrm{B}}$ & Parameter B of parabolic light response curve & - & Species-specific trait \\
\hline Ic & Parameter $\mathrm{C}$ of parabolic light response curve & - & Species-specific trait \\
\hline
\end{tabular}
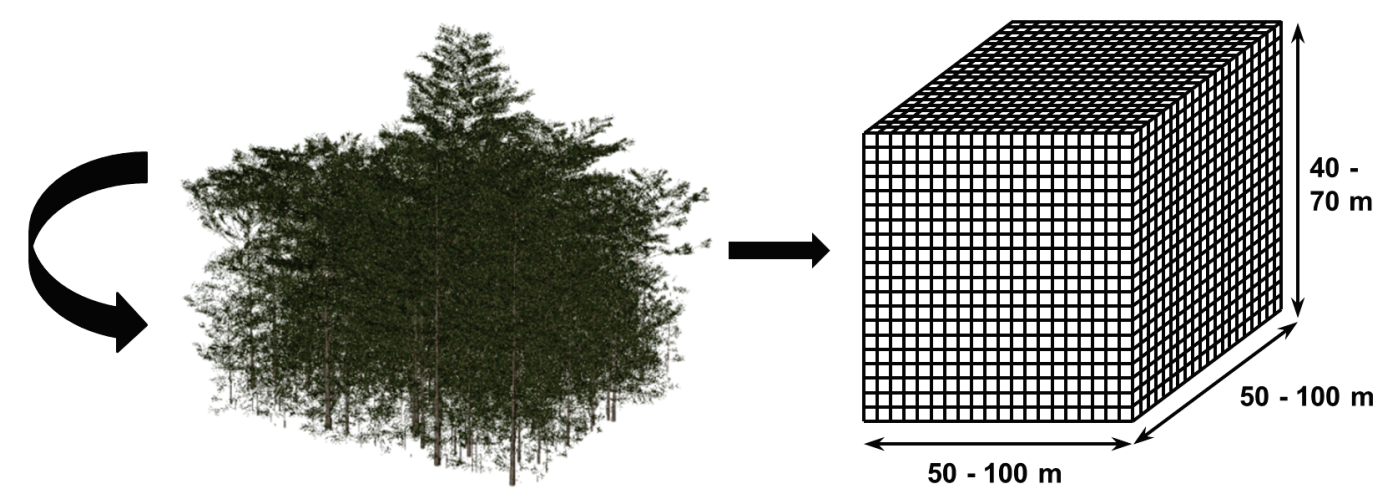

$50-100 \mathrm{~m}$

Figure 5.1. Generation of microhabitat matrices based on the result of the forest model. The left hand side illustrates a forest stand simulated with the FSPM. The spatial distribution of leaf area, branches and trunks is saved in each annual time step and on this basis the light distribution, total substrate area and relative annual loss of substrate area is calculated for each $1 \mathrm{~m}^{3}$ voxel in the microhabitat matrix (right hand side), which forms the basis of the epiphyte model. 


\section{Forest-epiphyte model}

\subsubsection{Process overview and scheduling}

The results of FSFMs at the stand scale (see chapter 4 for details) are used as input data for the epiphyte model. Based on these results, 3D microhabitat matrices containing the state variables of all voxel (Table 5.1) are calculated for each annual time step (Fig. 5.1). Using the initial microhabitat matrix, the initial distribution of individual epiphytes belonging to different species is generated (see section 5.3.1.5 for details). After initialization, the demographic processes recruitment, growth and mortality are simulated at each time step (Fig. 5.2). Each adult can reproduce at each time step, whereby the potential number of new recruits depends on the speciesspecific fecundity (Table 5.1) and the body mass of the individual in relation to its species-specific maximum mass. For each species, based on the position and potential number of recruits of each adult, as well as the species-specific 3D dispersal kernel and the available substrate area in each voxel, a probability matrix for establishment of new recruits is calculated. This matrix describes how many new recruit of a species could potentially establish in each of the voxels of the microhabitat matrix. The actual number of new recruits is then estimated based on Poisson random values. After recruitment, growth of each individual is simulated as a function of its mass and the light conditions in the specific voxel. In a last step, the mortality risk is estimated. Individuals die if the light conditions are outside the species-specific light niche or if they are the only occupier of a voxel whose surface area is too small to support the individual. In this case, we assume that the individual falls off the branch/stem. If several individuals occupy one voxel and their total space requirements exceed the available surface, smaller individuals are outcompeted by larger ones. Furthermore, individuals may die due to branch or tree fall, whereby the relative surface loss in a voxel defines the probability of mortality. In addition, body massdependent mortality probabilities, which follow the quarter-power law of the metabolic theory, are also used to account for causes of mortality not explicitly simulated. In the latter cases, random numbers between 0 and 1 are drawn to decide between death and survival. After this final step, the age of each surviving epiphyte is updated and the model proceeds with the next time step. 


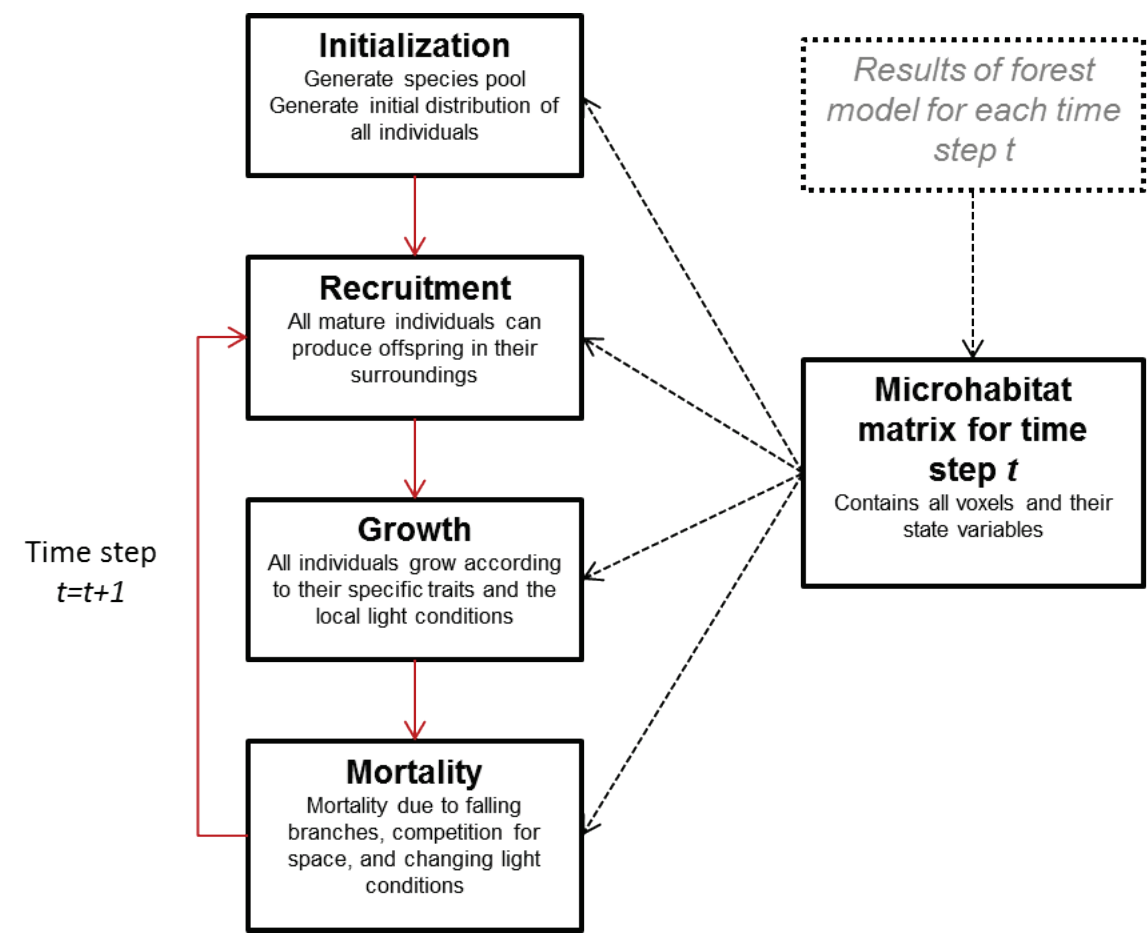

Figure 5.2. Flowchart of the coupled forest-epiphyte model. Based on the results of the FSFM, a microhabitat matrix characterizing the epiphytic habitat at each time step is generated. The initial spatial distribution of epiphytes and all demographic processes of individual epiphytes are influenced by the microhabitat matrices.

\subsubsection{Initialization}

The initial microhabitat matrix describes the light conditions and the distribution of substrate in each voxel and forms the basis of the initial epiphyte distribution. At first, a species pool containing traits of a defined number of epiphyte species (here: 100) is generated. Subsequently, the desired ratio of juvenile to mature plants in the initial assemblage is specified and, on this basis, an identical number of individuals per species is assigned to each group. Here, we used a total density of 400 individuals per species and hectare The maximum mass and the mass at maturity are species-specific traits, and based on these the initial mass of each juvenile and adult is randomly chosen from the range [0, mass at maturity] or [mass at maturity, maximum mass], respectively. Then, all individuals of all species are placed in the initial microhabitat matrix. Specifically, this means that for one individual after the other (to avoid biases, the sequence of individuals is randomized), all suitable voxels are estimated and one of these is randomly chosen as initial location. Suitable voxels have light conditions within the species-specific light niche (Table 5.1) and enough unoccupied surface area for the individual. The initial state of the model thus describes the location, state and species identity of each individual. This initial state is saved, which allows replications using identical initial conditions. 


\section{Forest-epiphyte model}

\subsubsection{Input}

The result of FSFMs simulated with the software GroIMP are used as input data in this model. In theses FSFMs, growth, establishment and mortality of three-dimensional virtual trees is simulated at the stand scale, whereby each tree consist of one trunk and branches up to the $2^{\text {nd }}$ order. Based on the 3D distribution and the dynamics of branches and trunks, the voxel-based distribution of substrate areas and their changes can be estimated. In addition, as the 3D distribution of leaf area is simulated at a resolution of $1 \mathrm{~m}^{3}$, the $3 \mathrm{D}$ light environment can also be calculated. These input data thus represent the dynamic of forests at a high level of detail.

\subsubsection{Submodels}

\section{Generation of microhabitat matrices}

A file containing the start position $P_{\mathrm{SStart}}{ }_{\mathrm{XYZ}}$ and end position $P_{\mathrm{SEnd}} \mathrm{XYZ}$ of each branch segment in 3D space, as well its length $L_{\mathrm{S}}$ and diameter $D_{\mathrm{S}}$, was saved at each time step in the FSFM (for details see chapter 4 ). Based on $L_{\mathrm{S}}$ and $D_{\mathrm{S}}$, the surface area of each branch segment $A_{\mathrm{S}}$ is calculated:

$$
A_{S}=\frac{\pi \cdot L_{S} \cdot D_{S}}{2}
$$

We assume that only the upper branch parts can be colonized by epiphytes, and hence the total surface area of the branch segment is divided by 2 . The maximum length of a branch segment is given by the maximum internode length used in the FSFM, which in this case was $0.5 \mathrm{~m}$. As the side length of a voxel is $1 \mathrm{~m}$, a branch segment may thus only intersect with a maximum of two voxels in $\mathrm{X}, \mathrm{Y}$ and $\mathrm{Z}$ direction. The intersecting voxels in $\mathrm{X}$ direction $V_{\mathrm{IntX}}$ are calculated as follows:

$$
V_{\text {IntX }}=\text { unique }\left(\operatorname{ceiling}\left(P_{\text {SStart }}{ }^{X}\right), \operatorname{ceiling}\left(P_{\text {SEnd }}{ }^{X}\right)\right)
$$

Analogously, the intersecting voxels in $\mathrm{Y}$ direction $V_{\text {IntY }}$ and $\mathrm{Z}$ direction $V_{\text {IntZ }}$ are estimated. Subsequently, the total number of intersecting voxel can be estimated as:

$$
n_{\text {Int }}=\operatorname{length}\left(V_{\text {IntX }}\right) \cdot \operatorname{length}\left(V_{\text {IntY }}\right) \cdot \operatorname{length}\left(V_{\text {IntZ }}\right)
$$


$A_{\mathrm{S}}$ is evenly split among all intersection voxels, i.e. the total surface area in these voxels is updated as:

$$
A_{B}=A_{B}+\frac{A_{S}}{n_{I n t}}
$$

In the vast majority of cases, branch segments are completely contained in a single voxel, or only intersect with two voxel, and thus we consider this simplified method as appropriate.

The position of each trunk in $\mathrm{X}$ and $\mathrm{Y}$ direction $P_{\mathrm{T}}{ }^{\mathrm{XY}}$, as well as its length $L_{\mathrm{T}}$ and diameter $D_{\mathrm{T}}$, are stored in a separate file. Trunks are not split into several segments in the FSTMs and their total surface areas thus have to be partitioned among intersecting voxel. To this end, we assume that each trunk has the form of a cone and only consider voxels directly above the voxel containing $P_{\mathrm{T}}{ }^{\mathrm{XY}}$. The highest voxel the trunk is intersecting with $V_{\mathrm{ZMax}}$ can easily be calculated based on $L_{\mathrm{T}}$ :

$$
V_{Z M a x}=\operatorname{ceiling}\left(L_{T}\right)
$$

For this voxel, the length of the intersecting trunk part $L_{\mathrm{TS}}$ is calculated as follows:

$$
L_{T S}=L_{T}-\text { floor }\left(L_{T}\right)
$$

As the radius in a cone linearly decreases with height, the radius at the intersection between the trunk part and the voxel $r_{\text {Int }}$ can be estimated as

$$
r_{I n t}=\frac{L_{T S} \cdot D_{S}}{L_{S} \cdot 2}
$$

Based on $r_{\mathrm{Int}}$ and $L_{\mathrm{TS}}$, the lateral surface of the cone in this voxel $A_{\mathrm{BT}}$ representing the surface area can be calculated:

$$
A_{B T}=\pi \cdot r_{I n t} \cdot \sqrt{r_{I n t}^{2}+L_{T S}^{2}}
$$

Analogously, the total lateral surface of the cone spanning from the intersection between the next lower voxel to the maximum tree height can be estimated. To calculate the surface area only intersecting with the specific voxel, $A_{\mathrm{BT}}$ has to be subtracted from this. Following this routine from the highest to the lowest voxel, the surface area in each voxel can be calculated. 


\section{Forest-epiphyte model}

Looping through the trunk and all branch segments, the total surface area in each voxel is calculated successively. To estimate the relative loss of surface at this time step, the list of all branch segments and trunks is compared with the list in the succeeding time step. As each of this tree parts has a unique identifier, it can easily be estimated if it got lost in this year. Following the same procedure as described above, the loss of surface area in each voxel is calculated, and by relating this loss to the total surface area, the percentage annual loss $S_{\text {Loss }}$ is estimated.

The light intensity in each voxel is calculated based the total leaf area in each voxel which was saved at each time step in the FSFM. Based on the sum of $A_{\mathrm{LTot}}{ }^{\mathrm{XYZ}}$ in all voxels above the specified voxel, the leaf area index $L A I^{\mathrm{XYZ}}$ for each voxel is calculated.

$$
L A I^{X Y Z}=\frac{\sum_{z}^{M a x Z} A_{L t o t}{ }^{X Y Z}}{L_{V}{ }^{2}}
$$

where $L_{\mathrm{V}}$ is the side length of a voxel. Assuming a Lambert-Beer extinction law, the singlecolumn light intensity $I_{\mathrm{SC}}{ }^{\mathrm{XYZ}}$ is calculated based on $L A I^{\mathrm{XYZ}}$.

$$
I_{S C}{ }^{X Y Z}=I_{C} \cdot e^{-\left(k_{L} \cdot L A I^{X Y Z}\right)}
$$

where $I_{\max }$ is the light intensity above the canopy and $k_{\mathrm{L}}$ the light extinction coefficient. This method assumes that solar radiation only penetrates directly from above and disregards additional processes like light reflection. This is an oversimplification, particularly in such heterogeneous forests as simulated here. To get a more realistic estimation of the average, effective light intensity within a voxel $I^{\mathrm{XYZ}}$, the single column light intensity $I_{\mathrm{SC}}{ }^{\mathrm{XYZ}}$ in the voxels surrounding the focal voxel in $\mathrm{x}$ and $\mathrm{y}$ direction are additionally taken into account. The number of surrounding voxels considered depends on the parameter $L R$ which defines how many rectangular rings around the focal voxel are considered. For each considered voxel, the relative contribution $C_{\mathrm{R}}$ is calculated, with $\Sigma C_{\mathrm{R}}=1 . C_{\mathrm{R}}$ thus defines how strong $I_{\mathrm{SC}} \mathrm{XYZ}$ in each voxel contributes to $I^{\mathrm{XYZ}}$ and we assume that all rings contribute equally:

$$
C_{R}=\frac{1}{L R+1} \cdot \frac{1}{\max (1, R \cdot 8)}
$$


On this basis, $I^{\mathrm{XYZ}}$ is calculated as

$$
I^{X Y Z}=\sum_{X_{\min }}^{X_{\max }} \sum_{Y_{\min }}^{Y_{\max }} I_{S C}{ }^{X Y Z} \cdot C_{R}
$$

where $X_{\min }=X$-LR and $X_{\max }=X+L R$ (likewise for $Y$ ).

\section{Generation of initial species pool}

At the beginning of each simulation, the local species pool containing the trait information of $n_{\mathrm{Sp}}$ species is initialized. Each species has a unique identifier and is characterized by a set of traits (Table 5.1). Some traits are randomly chosen from defined ranges (Table 5.2), others are related to the body mass following the principles of the metabolic theory of ecology (MTE; Brown et al. 2004).

Table 5.2. Parameter ranges of the epiphyte model.

\begin{tabular}{llll}
\hline Symbol/parameter & Description & Unit & Range \\
\hline$M_{\text {Max }}$ & Maximum mass of species & $\mathrm{g}$ & {$[2,1000]$} \\
$M_{\text {Rel }}$ & Relative mass in relation to maximum mass at which & $\mu \mathrm{mol} \mathrm{m}^{-2} \mathrm{~s}^{-1}$ & {$[0.5,0.8]$} \\
& maturity is reaches & & \\
$D_{\mathrm{K}}$ & Factor b in negative exponential function & - & {$[0.1,0.8]$} \\
$D_{\mathrm{KAs}}$ & Dispersal kernel asymmetry & - & {$[0.5,0.9]$} \\
$H_{\text {Mean }}$ & Mean height relative to forest height & - & {$[0,1]$} \\
$H_{\text {Range }}$ & Height range (relative) & & {$[0.2,0.8]$} \\
$\mathrm{n}_{\mathrm{R}}$ & Average number of recruits per mature plant & & {$[5,10]$} \\
\hline
\end{tabular}

First, the maximal body mass $M_{\text {Max }}$ of each species is specified based on the defined lower and upper limits $M_{\mathrm{Max}}{ }^{\mathrm{L}}$ and $M_{\mathrm{Max}}{ }^{\mathrm{U}}$ (the superscripts in this section always refer to the user-defined upper and lower limits of a specific parameter; see Table 5.2). We assume that smaller species are more frequent and thus chose $M_{\mathrm{Max}}$ randomly from the uniform distribution after $\log$ transformation of $M_{\mathrm{Max}}{ }^{\mathrm{L}}$ and $M_{\mathrm{Max}}{ }^{\mathrm{U}}$ :

$$
M_{\text {Max }}=10^{\mathrm{unif}\left(\log \left(M_{\text {Max }}{ }^{L}\right), \log \left(M_{\text {Max }}{ }^{U}\right)\right)}
$$




\section{Forest-epiphyte model}

We assume that the mass at maturity $M_{\text {Mat }}$ scales with $M_{\text {Max }}$ :

$$
M_{M a t}=M_{M a x} \cdot \operatorname{unif}\left(M_{R e l}{ }^{L}, M_{R e l}^{U}\right)
$$

where $M_{\text {Rel }}$ defines the ratio between $M_{\text {Mat }}$ and $M_{\text {Max }}$. The MTE predicts a positive quarter-power scaling of the age at maturity $A_{\text {Mat }}$ with $M_{\text {Max }}$ (Brown et al. 2004; Duncan, Forsyth \& Hone 2007), and accordingly $A_{\text {Mat }}$ is calculated as follows:

$$
A_{\text {Mat }}=\left(k_{\text {Mat }}+A_{\text {Mat }}{ }^{1 / 4}\right) \cdot \operatorname{unif}\left(A_{\text {MatDev }}{ }^{L}, A_{\text {MatDev }}{ }^{U}\right)
$$

where $\mathrm{k}_{\mathrm{Mat}}$ is the intercept of the $A_{\mathrm{Mat}}-M_{\mathrm{Max}}$ relationship. To add stochasticity, the relative deviation from the mean trend $A_{\text {MatDev }}$ is also considered.

In this model, epiphytes grow according to the Bertalanffy growth law (see subsection growth), in which the growth rate (or curvature parameter) $K$ is a species-specific parameter. This parameter can be calculated based on $M_{\text {Max }}, M_{\text {Mat }}$ and $A_{\text {Mat }}$ as follows:

$$
K=-\left(\frac{\log (1)+\log \left(1-\frac{M_{\text {Mat }}}{M_{\text {Max }}}\right)}{A_{\text {Mat }}}\right)
$$

In natural epiphyte communities, a pronounced vertical stratification is usually observed (Krömer et al. 2007; Petter et al. 2016). The reasons why species occurrences are limited to specific vertical ranges are complex; however, in this model we assume that light defines the niche. In a forest canopy, the light intensity does not vary linearly from the upper strata to the forest floor, but rather non-linearly, commonly described by a light-attenuation law such as Lambert-Beer. Hence, the height niche and the light niche are not linearly related. As vertical niches of epiphyte species are better studied and more intuitive, we at first specify the vertical niche for each species in a standardized forest (Optimum height, minimum height and maximum height relative to the maximum height of the forest), and subsequently translate the vertical niche to the light niche. Thus, at first the relative mean height $H_{\text {Mean }}$ is randomly chosen for each species:

$$
H_{\text {Mean }}=\operatorname{unif}(0,1)
$$


$H_{\text {Mean }}$ defines the mean height of the vertical niche relative to the maximum height of a standardized forest, and thus may vary between 0 and 1 . The breadth of the vertical niche $H_{\text {Range }}$ is randomly chosen from the defined ranges $H_{\text {Range }}$ :

$$
H_{\text {Range }}=\operatorname{unif}\left(H_{\text {Range }}{ }^{L}, H_{\text {Range }}{ }^{U}\right)
$$

The lower and upper boundary of the vertical niche can be determined based on $H_{\text {Mean }}$ and $H_{\text {Range }}$ as follows:

$$
\begin{aligned}
& H_{\text {Min }}=\max \left(0, H_{\text {Mean }}-\frac{H_{\text {Range }}}{2}\right) \\
& H_{\text {Max }}=\min \left(1, H_{\text {Mean }}+\frac{H_{\text {Range }}}{2}\right)
\end{aligned}
$$

$H_{\text {Min }}$ and $H_{\text {Max }}$ are thus truncated when exceeding the natural vertical limits of 0 and 1 . Based on the upper and lower limits of the vertical niche, the limits of the light niche are estimated as follows:

$$
\begin{aligned}
& I_{\text {Min }}=I_{C} \cdot e^{-\left(k_{L} \cdot L A I *\left(1-H_{\text {Min }}\right)\right)} \\
& I_{\text {Max }}=I_{C} \cdot e^{-\left(k_{L} \cdot L A I *\left(1-H_{\text {Max }}\right)\right)}
\end{aligned}
$$

where $I_{\mathrm{C}}$ is the average light intensity above the canopy and the LAI is the leaf area index in the hypothesized forest. We assume that the light optimum of each species lies midway between $I_{\text {Min }}$ and $I_{\mathrm{Max}}$ :

$$
I_{O p t}=\frac{I_{M i n}+I_{M a x}}{2}
$$

$I_{\text {Min }}$ and $I_{\text {Max }}$ define the light intensities under which a species can survive. However, it would be too simplistic to assume that the growth of a species would be constant under all light conditions in the niche. Thus, we assumed that growth would be maximal under optimal light conditions $I_{\mathrm{Opt}}$, and is reduced when deviating from this optimum. We use a parabolic growth response to simulate this situation (see submodel growth), whereby the vertex of the parabola is given by $I_{\mathrm{Opt}}$ and $I_{\text {Min }}$ and $I_{\mathrm{Max}}$ define the points at which the growth response becomes zero.

The parameters of this growth response function $(a, b$ and $c)$ are thus species-specific parameters that are calculated as follows: 


$$
\begin{aligned}
& a=\frac{I_{\text {Max }}-I_{\text {Min }}}{\left(I_{\text {Min }}-I_{\text {Max }}\right) \cdot\left(I_{\text {Min }}-I_{O p t}\right) \cdot\left(I_{O p t}-I_{\text {Max }}\right)} \\
& b=\frac{I_{\text {Max }}{ }^{2}-I_{\text {Min }}{ }^{2}}{\left(I_{\text {Min }}-I_{\text {Max }}\right) \cdot\left(I_{\text {Min }}-I_{O p t}\right) \cdot\left(I_{O p t}-I_{\text {Max }}\right)} \\
& \mathrm{c}=\frac{\left(I_{\text {Min }}{ }^{2} \cdot I_{\text {Max }}\right)-\left(I_{\text {Min }} \cdot I_{\text {Max }}{ }^{2}\right)}{\left(I_{\text {Min }}-I_{\text {Max }}\right) \cdot\left(I_{\text {Min }}-I_{O p t}\right) \cdot\left(I_{O p t}-I_{\text {Max }}\right)}
\end{aligned}
$$

Now, the species-specific parameters related to dispersal and reproduction are defined. We use a negative exponential function to describe the dispersal kernel (see section establishment), and the species-specific parameter $D_{\mathrm{K}}$ describing the shape of the kernel is randomly chosen from the uniform distribution on the interval $\left[D_{\mathrm{K}}{ }^{\mathrm{L}}, D_{\mathrm{K}}^{\mathrm{U}}\right]$ :

$$
D_{K}=\operatorname{unif}\left(D_{K}{ }^{L}, D_{K}{ }^{U}\right)
$$

Dispersal kernels define the probability of dispersal as a function of distance from the source. They are mainly use in one- or two-dimensional space. A simple application of common kernels in three-dimensional space might not be adequate, as due to the effect of gravity downward dispersal is more probable than upward dispersal. To account for this effect, we additionally defined the species-specific trait dispersal kernel asymmetry $D_{\mathrm{KAs}}$, which is randomly chosen as follows:

$$
D_{K A s}=\operatorname{unif}\left(D_{K A S}{ }^{L}, D_{K A S}{ }^{U}\right)
$$

This traits describes the probability that seeds are dispersed below the mother plant; hence $D_{\mathrm{KA}}=0.5$ describes a symmetric dispersal in all direction (for more details see section establishment).

Finally, traits related to the fecundity of the species are defined, and the average number of recruits per mature plant $n_{\mathrm{R}}$ is randomly chosen based on the defined ranges as follows:

$$
n_{R}=\operatorname{unif}\left(n_{R}{ }^{L}, n_{R}{ }^{U}\right)
$$

This model does not separate the processes seed dispersal, germination and seedling establishment; $n_{\mathrm{R}}$ can thus be understood as number of seedlings from a single mother plant that could establish in one year if substrate area of a sufficient size ( $1 \mathrm{~m}^{3}$ of substrate per voxel) would be available in the surroundings of the mother. 
As the entire process of recruitment of epiphytes is complex and not well-studied, we did not integrate an effect of the body mass of the species on the number of recruits, i.e. both small and large plants can have similar $n_{R}$ in our model. However, Zotz (1998) observed that within a species, the number of fruits/seedlings increase with size of the epiphyte. To account for this, we integrate a species-specific trait $n_{\text {RInc }}$ that is randomly chosen as follows:

$$
n_{R I n c}=\operatorname{unif}\left(n_{R I n c}{ }^{L}, n_{R I n c}{ }^{U}\right)
$$

This trait describes the ratio between the number of recruits at maximum mass and the number of recruits at the mass at maturity, i.e. an $n_{\text {RInc }}=2$ means that a at maximum mass the number of recruits is twice as high as at mass at maturity.

\section{Generation of initial distribution}

Based on the local species pool, an initial spatial distribution of the epiphyte assemblage is generated. First, the number of individuals per species and ha $\left(n_{\mathrm{Ha}}\right)$ and the ratio of juvenile to mature plants $\left(r_{\mathrm{MJ}}\right)$ are defined (here: $n_{\mathrm{Ha}}=400 \mathrm{ha}^{-1}$ and $r_{\mathrm{MJ}}=0.5$ ). Subsequently, a list containing all individuals in the assemblage is generated, and individuals of each species are divided into juveniles/adults according to $r_{\mathrm{MJ}}$. For each juvenile, the initial body mass $M$ is randomly chosen as follows:

$$
M=\operatorname{unif}\left(\frac{M_{M a t}}{100}, M_{M a t}\right)
$$

The initial mass of each adult is estimated accordingly:

$$
M=\operatorname{unif}\left(M_{M a t}, M_{M a x}\right)
$$

The positions in the list containing all individuals are randomly shuffled, and following this order each individual is distributed on the initial microhabitat matrix. Specifically, this means that, at first, all voxels having light conditions within the species-specific light niche ( $\left.I_{\mathrm{Min}}, I_{\mathrm{Max}}\right)$ are selected as potential habitat. Subsequently, voxels whose unoccupied surface area is smaller than the space requirements of the individual are excluded. The space requirements are calculated as follows:

$$
S=M^{2 / 3} \cdot g_{S}
$$




\section{Forest-epiphyte model}

where $g_{\mathrm{S}}$ is a scaling parameter. This means, we assume that the space occupied by an individual scale with its mass to the power $2 / 3$, and $g_{\mathrm{S}}$ relates $M^{2 / 3}$ to occupied surface area. Finally, after all potential voxels have been identified, a single one is randomly selected as initial location of the individual and the total occupied surface area in this voxel is updated accordingly. This procedure is repeated for all individuals. If there should be no suitable voxel for an individual, this is recorded and thus allows evaluating the adequacy of the initial distribution before starting the actual simulation.

\section{Recruitment}

Recruitment in each time step is based on 3D probability matrices. To calculate these matrices, $3 \mathrm{D}$ distance matrices are calculated at first (dimension in $\mathrm{X}$ direction $=(2 \operatorname{MaxX})+1$; in $\mathrm{Y}$ and $\mathrm{Z}$ direction accordingly). The Euclidian distance to the center of the matrix is calculated for each voxel of these matrices. Based on the distances, the probability for dispersal in each voxel is calculated using the dispersal kernel which is described by a negative exponential function:

$$
P_{D R}=\mathrm{e}^{-D_{V} \cdot D_{K}}
$$

where $D_{\mathrm{V}}$ is the distance to the central voxel and $D_{\mathrm{K}}$ is the species-specific dispersal parameter. We assume that the dispersal kernel is not symmetric in $\mathrm{Z}$ direction, and the species-specific asymmetry is defined by $D_{\mathrm{KA}}$. The probabilities of dispersal in all voxel above the central voxel are thus modified as follows:

$$
P_{D}=P_{D R} \cdot 2 \cdot\left(1-D_{K A}\right)
$$

Accordingly, the probabilities of dispersal in all voxel below the central voxel are modified:

$$
P_{D}=P_{D R} \cdot 2 \cdot D_{K A}
$$

After $P_{\mathrm{D}}$ for all voxels are calculated, the probabilities are normalized so that: $\sum P_{\mathrm{D}}=1$.

Now, for each mature individual in the community, the potential number of recruits is calculated as follows:

$$
n_{R P o t}=n_{R} \cdot n_{R I n c} \cdot \frac{M-M_{\text {Mat }}}{M_{\text {Mat }}-M_{\text {Max }}}
$$


Subsequently, for each adult, the probability matrix is multiplied with $n_{\mathrm{RPot}}$ and a sub-matrix of this probability matrix is generated. This sub-matrix has the same dimensions as the microhabitat matrix (MaxX, MaxY, MaxZ) and is selected in such a way that the 3D position of the individual epiphyte matches the central voxel of the probability matrix (This sub-setting step is the reason for the doubled dimensions of the probability matrix as this allows to generate an adequate subset at all possible position of epiphyte in the microhabitat matrix). This matrix is multiplied with the surface matrix and the niche matrix, which describes suitable voxel with a 1 and unsuitable ones with a 0 . The resulting final matrix describes how many new recruit of a species could potentially establish in each of the voxels of the microhabitat matrix. The actual number of new recruits is then estimated based on Poisson random values.

\section{Growth}

Growth of each individual is simulated as follows:

$$
M_{(t+1)}=M(t 0)+k \cdot\left(M_{M a x}-M_{(t 0)}\right) \cdot\left(a \cdot I_{V}^{2}+b \cdot I_{V}+c\right)
$$

This equation combines a Bertalanffy growth function, in which the growth rate declines with increasing body mass, and a parabolic light-response function.

\section{Mortality}

First, the mortality due to branch fall is simulated based on the relative loss of surface in the voxel of the individual. We assume that the probability that an individual falls attached to a branch equals the relative loss rate of surface area. This means, if $30 \%$ of the area in a voxel is lost due to branchfall, the mortality probability is $m_{\mathrm{BF}}=0.3$. For each individual, random numbers are drawn from the uniform distribution on the interval $[0,1]$ and, on this basis, mortality is determined.

Second, individuals die when light conditions are outside their light niches.

Third, we use a mortality rate following the MTE to account for cause of mortality not considered in this model. In this case, the mortality probability scales with the mass of an individual and is calculated as follows:

$$
m_{M T E}=k_{M}+M^{-1 / 4}
$$




\section{Forest-epiphyte model}

where $\mathrm{k}_{\mathrm{M}}$ is the intercept of this scaling function. For each individual, random numbers are drawn from the uniform distribution on the interval $[0,1]$ and, on this basis, mortality is determined.

Fourth, mortality due to competition for space is simulated. If the total surface area in a voxel is lower than the space required by all epiphytes in the voxel, the smallest individual is removed successively until the space requirements are fulfilled. This procedure is only applied to voxel with at least two individuals.

Fifth, if a voxel contains a single individual whose space requirements cannot be fulfilled, we assume that this individual falls off the branch.

All dead individuals are removed from the community, the age of all surviving ones are updated and the model proceeds with the next time step.

\subsubsection{Model validation and scenarios}

\section{Model parameterization and validation}

The main objective in this study is to analyze how differences in forest dynamics affect the structure and dynamics of epiphyte assemblages based on different simulation experiments. In chapter 4 , we simulated a structurally realistic Neotropical lowland forest, and this forest model constitutes our reference scenario. In order to compare epiphyte assemblage on this reference forest with other forest scenarios, a realistic epiphyte model that reproduces the dynamics and structure of natural epiphyte communities is required.

Our knowledge on the long-term dynamics of epiphyte assemblages is very limited, i.e. data to which we could fit the model is not available. However, we can assume that an epiphyte community should be in a dynamic equilibrium state in the long term. In addition, Zotz et al. (1999) observed that most epiphyte assemblages show no signs of 'saturation'; thus a nonsaturated assemblage in dynamic equilibrium state can be assumed.

In contrast to the dynamics, the structure of epiphyte assemblages is better known. In this study we had access to two independent datasets from Panama and Ecuador, in which the 3D epiphyte distribution was observed (Panama: 0.4 ha, see Zotz \& Schultz 2008 for details; Ecuador: 0.1 ha, unpublished data by H. Kreft and N. Köster). All individuals in these datasets were identified to the species level. For each dataset, we analyzed the vertical distribution of individuals, the vertical stratification of species, the size distribution and rank-abundance curves. These ecological patterns were used to parameterize and validate our model. 
We began by generating a number of the different species pools which we simulated on the reference forest over 100 years. The average population growth rates for these species were estimated and used to assess their fitness; species with exploding populations or those which go extinct are not appropriate to be included in the final model. By assessing population growth rates and adjusting the parameters according, we were able to obtain species pools with many suitable species. However, as a few dominant species in a species pool with very high population growth rates can still have a strong effect on the dynamics of the assemblage, we only included species whose average population growth rates were between 1 and 1.01 in the pool of suitable species. On this basis, we generated ten local species pools containing 100 suitable species each, and simulated their dynamics over 600 years on five replicates of the reference forest. The suitability of these simulations was evaluated by comparing the resulting ecological patterns with those observed in the empirical datasets.

\section{Simulation experiments}

After we identified ten sets of species pools which simulated suitable epiphyte assemblages on the reference forest, we simulated these species pools on a number of different forest scenarios.

In the first simulation experiment, we assess the effect of differences in natural forest dynamics on the dynamics of the epiphyte assemblages. We generated three forest scenarios in addition to the reference forest, which differed in their stem turnover rates. These scenarios are referred to as high-turnover scenario, low-turnover scenario and very-low-turnover scenario. The dynamics of twelve important forest attributes in these scenarios are illustrated in Fig. D.1. In addition, we generated a scenario in which the reference forest is stable and does not show any dynamics. In other words, the forest stays as it is in the initial time step and no branches or trees ever die.

In the second simulation experiment, we assess the effect of selective logging on the dynamics of the epiphyte assemblages. We generated three different logging scenarios which differ in the diameter at which the trees are logged. These scenarios are referred to as logging40, logging 45 and logging50 scenario according the minimum diameter for logging (see Fig. D. 2 to compare these scenarios with the reference forest)

In the third simulation experiment, we assess the effect of fragment size on the dynamics of the epiphyte assemblages. We simulated the references forest at three different spatial scales $(0.25$, 0.5 and 1 ha; see Fig. D.3). Epiphyte density was identical in all initial epiphyte assemblage, i.e. 400 individuals per species and ha. 


\section{Forest-epiphyte model}

\subsection{Results}

\section{Model validation}

Long-term dynamics of 10 different initial epiphyte assemblages on five replicates of a typical lowland forest are shown in Fig. D.4, and the averaged dynamics in each forest replicate are presented in Fig. 5.3. The simulations indicate that the assemblages reached a dynamic equilibrium state, but fluctuations in abundance were pronounced (Fig. 5.3a). These fluctuations were more influenced by differences in forest dynamics among forest replicates than by differences in the initial epiphyte species pool (Fig. D.4a). Annual community growth rates ranged from $\sim 0.9$ to $\sim 1.05 \mathrm{a}^{-1}$ (see example in Fig. D.5a). Drastic short-term losses in abundance, caused by the fall of larger trees, were thus compensated by positive community growth rates in periods without substantial tree mortality events (Fig. D.5a). Overall, mean annual mortality rates of $\sim 14 \%$ were observed (Table D.1). On average, $3.6 \% \mathrm{a}^{-1}$ of all individuals fell to the ground attached to branches, and $2.4 \% \mathrm{a}^{-1}$ fell off branches (Table D.1). Mortality due to competition $\left(0.9 \% \mathrm{a}^{-1}\right)$ or due to changing environmental conditions following changes in forest structure $\left(0.5 \% \mathrm{a}^{-1}\right)$ was less important. We also used a mass-dependent mortality rate following the principles of the metabolic theory to account for causes of mortality not explicitly simulated, and this mortality rate was approximately $6.9 \% \mathrm{a}^{-1}$. All species survived the initial $\sim 50-80$ years, but subsequently some species went locally extinct (Fig. 5.3b).
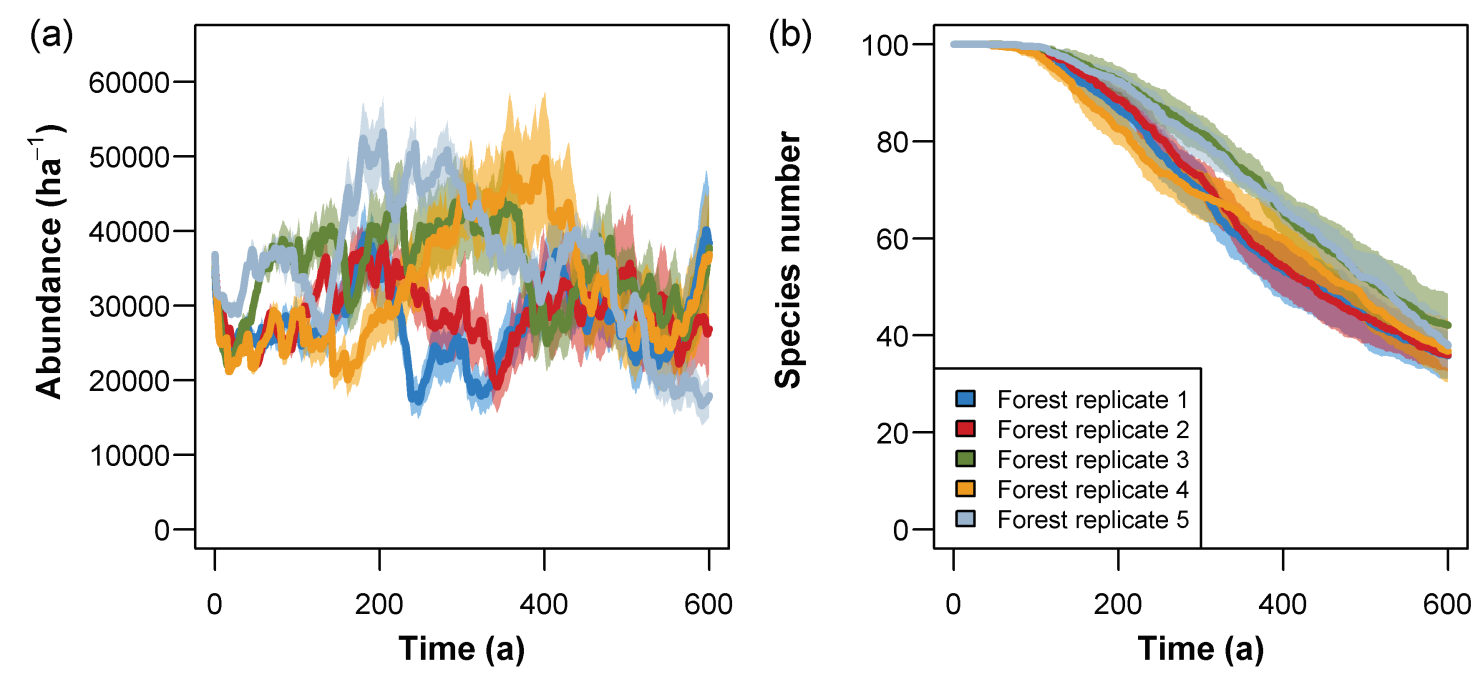

Figure 5.3. Simulated long-term dynamics of vascular epiphyte communities. Five replicates of a typical lowland forest stand $(50 \times 50 \mathrm{~m})$ were simulated (see Fig. D.1 for forest attributes) and used as input data for the epiphyte model. On each of these forest replicates, the development of epiphyte communities, which initially consisted of 100 individual of 100 species, was simulated over 600 years. Ten different initial species pools were simulated on each forest replicate and means (bold lines) and standard deviations (shaded areas) of abundance (a) and species numbers (b) are shown. 
Starting from an even initial distribution (100 individuals per species), our model simulated typical right-skewed rank-abundance distributions. However, deviations occurred at the tails of the distributions compared to the two reference epiphyte assemblages (Fig. 5.4a). This means that rare species represented by only few individuals (i.e. singletons) were underrepresented in comparison to the reference assemblages.

In the dynamic equilibrium state, epiphytes were not evenly distributed along the vertical axis of the canopy. Rather, relative abundance peaked in the canopy zone between 20 and $30 \mathrm{~m}$ in later time periods (Fig. 5.4b). At the beginning of each simulation, species were randomly distributed on available substrate within their potential light niches (also see Fig. 5.5a) and the initial vertical distributions of epiphyte assemblage resembled the vertical distribution of available substrate in the forest (compare panels a, $d$ and $g$ with panel $\mathrm{j}$ in Fig. D.6). However, in almost all simulations, a clear shift in relative abundance with time towards higher zones of the canopy was observed (Fig. D.6c,f,i). The temporal changes in 3D distribution are additionally visualized in comparison to the reference assemblages in Fig. D.7.
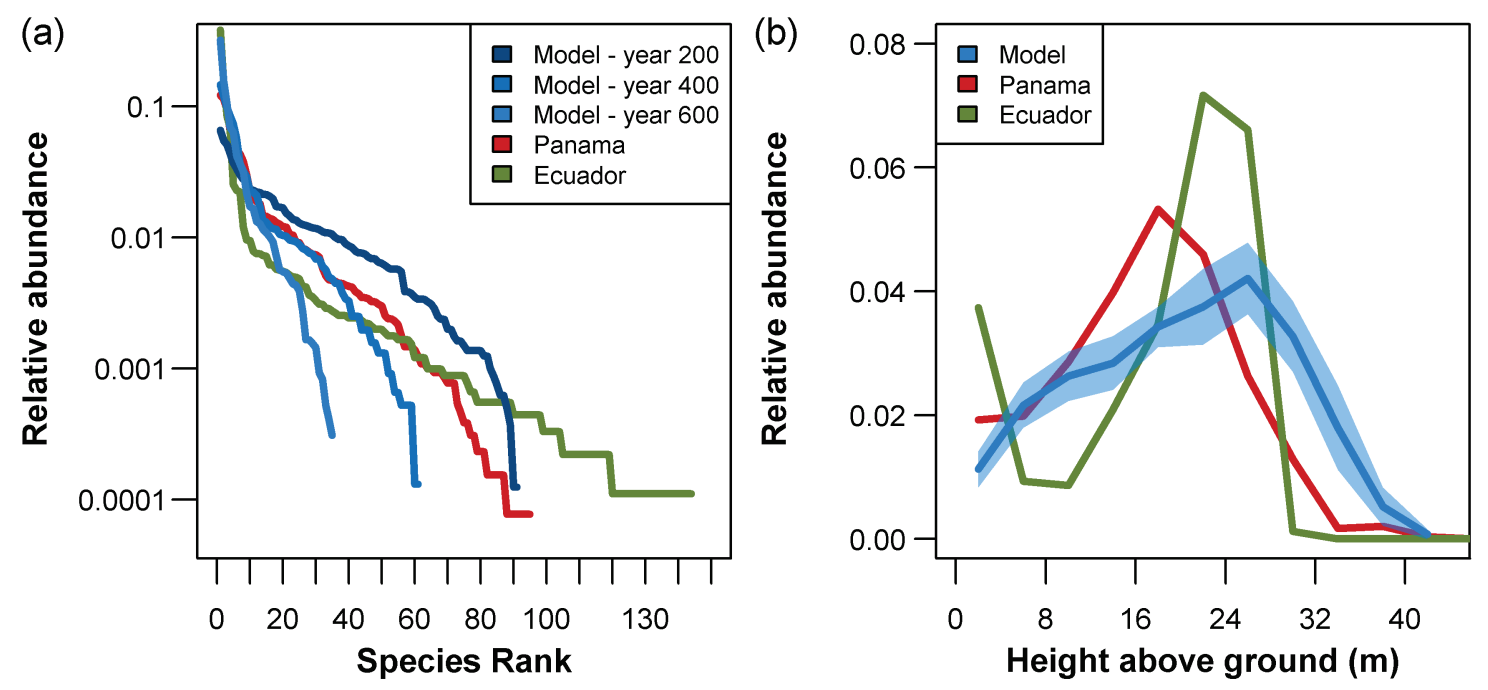

Figure 5.4. Rank-abundance curves and vertical distributions of epiphytes: (a) Relative abundances of species sorted by their abundance rank in descending order in one representative model run at several time steps in comparison to empirical data from rainforests in Panama and Ecuador. (b) Simulated vertical distribution of epiphytes in comparison to empirical data from Panama and Ecuador.

The simulated vertical stratification of species resembled observed patterns in the reference assemblages (Fig. 5.5). However, the variability in height ranges was less pronounced and particularly such narrow height ranges of low trunk specialist, which were abundant in the Ecuadorian plot, were not simulated. 

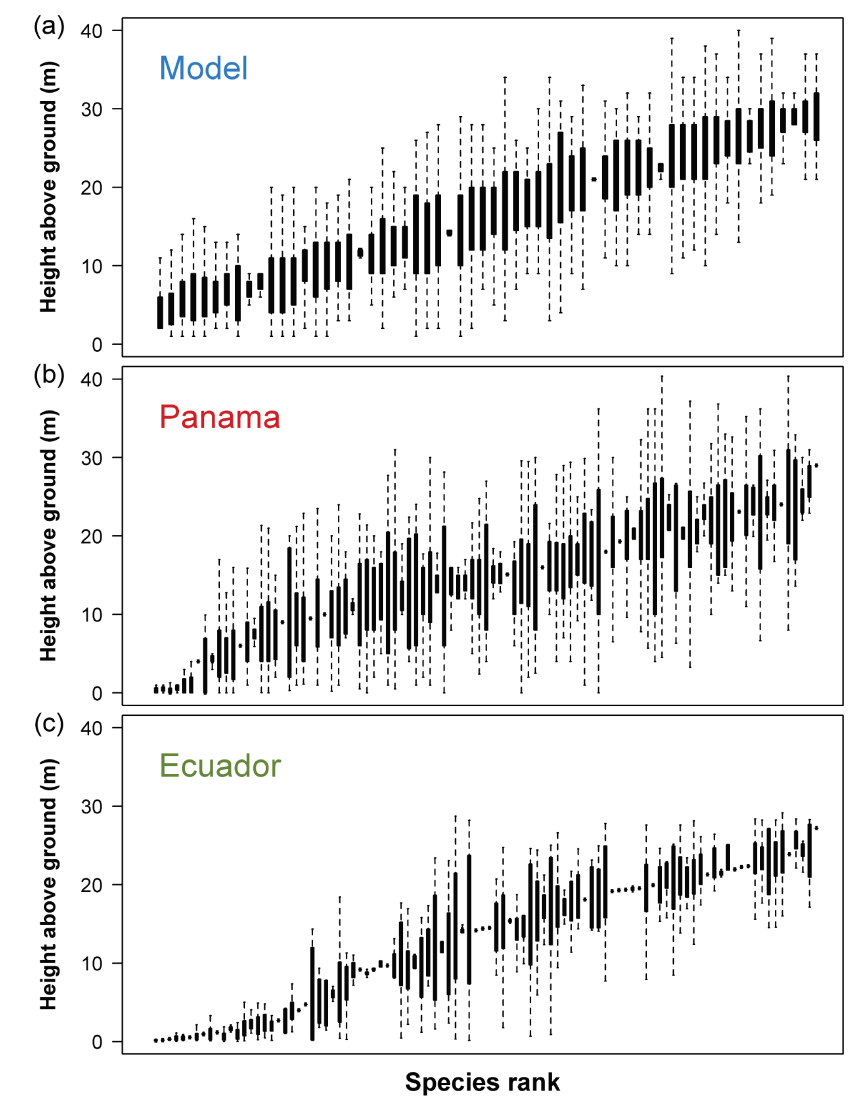

Figure 5.5. Vertical stratification (measured as height above ground) of species in our model (a) in comparison to data from Panama (b) and Ecuador (c). The simulated height distribution after 300 years in one representative model run is shown.

The simulated assemblage was clearly size-structured and dominated by smaller individuals (Fig. 5.6a). Averaged over all forest replicated and time steps, the epiphyte biomass was approximately $350 \mathrm{~kg}$ per ha.

(a)

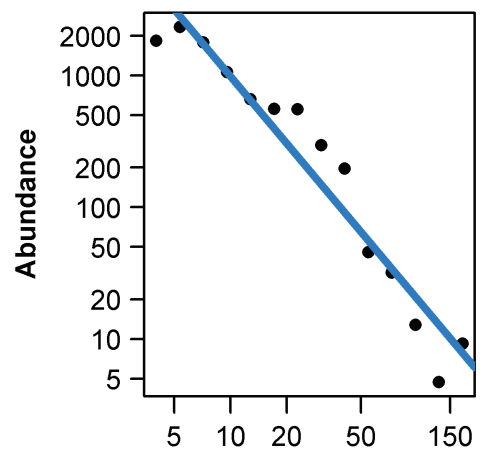

Plant mass (g) (b)

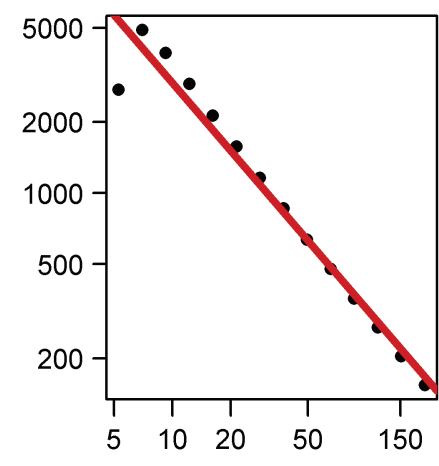

Leaf length $(\mathrm{cm})$ (c) Ecuador

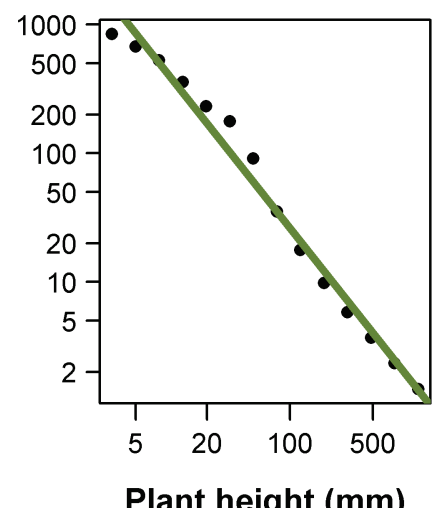

Figure 5.6. Size-distributions in epiphyte communities: (a) Model, (b) Panama, (c) Ecuador. 


\section{Simulation experiments}

Differences in natural forest dynamics clearly influenced the abundance, diversity and 'saturation level' of the epiphyte community (Fig. 5.7a,b,c). The percentage of arboreal substrate occupied by epiphytes, which we used to characterize the saturation level of the epiphyte assemblage, reached relatively stable levels that were clearly distinguishable between the different forest scenarios (Fig. 5.7c). A stable, non-dynamic forest was used as reference, and in this scenario the epiphyte community occupied $\sim 40 \%$ of total available substrate area. There are two main reasons why this level represents a "saturated" community. On the one hand, we assumed that epiphytes can only occupy the upper branch surface. On the other hand, we used a voxel-based approach in which individuals were removed from a voxel if their total space requirement exceeded the available surface area in a voxel (space competition), and thus the surface in voxel is normally not completely filled. The reference saturation level was almost reached in the forest scenario with very low tree turnover rates, but all other scenarios were below this level and their sequence matched tree turnover rates, with decreasing saturation levels with increasing turnover rates (Fig. 5.7c). Epiphyte abundances showed similar patterns for the non-saturated forests, but in the saturated forest the abundance decreased over time (Fig. 5.7a). This decrease is due to the assumed size-asymmetric competition whose effects increased with saturation. The generally higher abundances in the scenario with very low tree turnover rates compared to the reference scenario can be explained by the larger arboreal surface areas, owing to a higher density of large trees (Fig. D.1). Rates of local species extinctions also differed among forest scenarios and the number of species surviving until the end of the simulation was clearly influenced by stem turnover (Fig. 5.7b). Interestingly, species numbers in the low and very low turnover scenarios were almost identical, although effects on abundance were consistent.

Selective logging of larger trees resulted in lower abundances, species numbers and saturation levels (Fig. 5.7d,e,f). Interestingly, a reduction in minimum tree size for logging from 45 to 40 $\mathrm{cm}$ had catastrophic effects on the epiphyte assemblage and resulted in a near-complete extinction of the epiphyte assemblage.

The fragment size clearly influenced species extinction rates (Fig. 5.7h). Differences in abundance and saturation levels in turn were less pronounced and not consistent (Fig. 5.7g,i). The increased abundance at the 1 ha plot might, however, be related to the higher species numbers. 

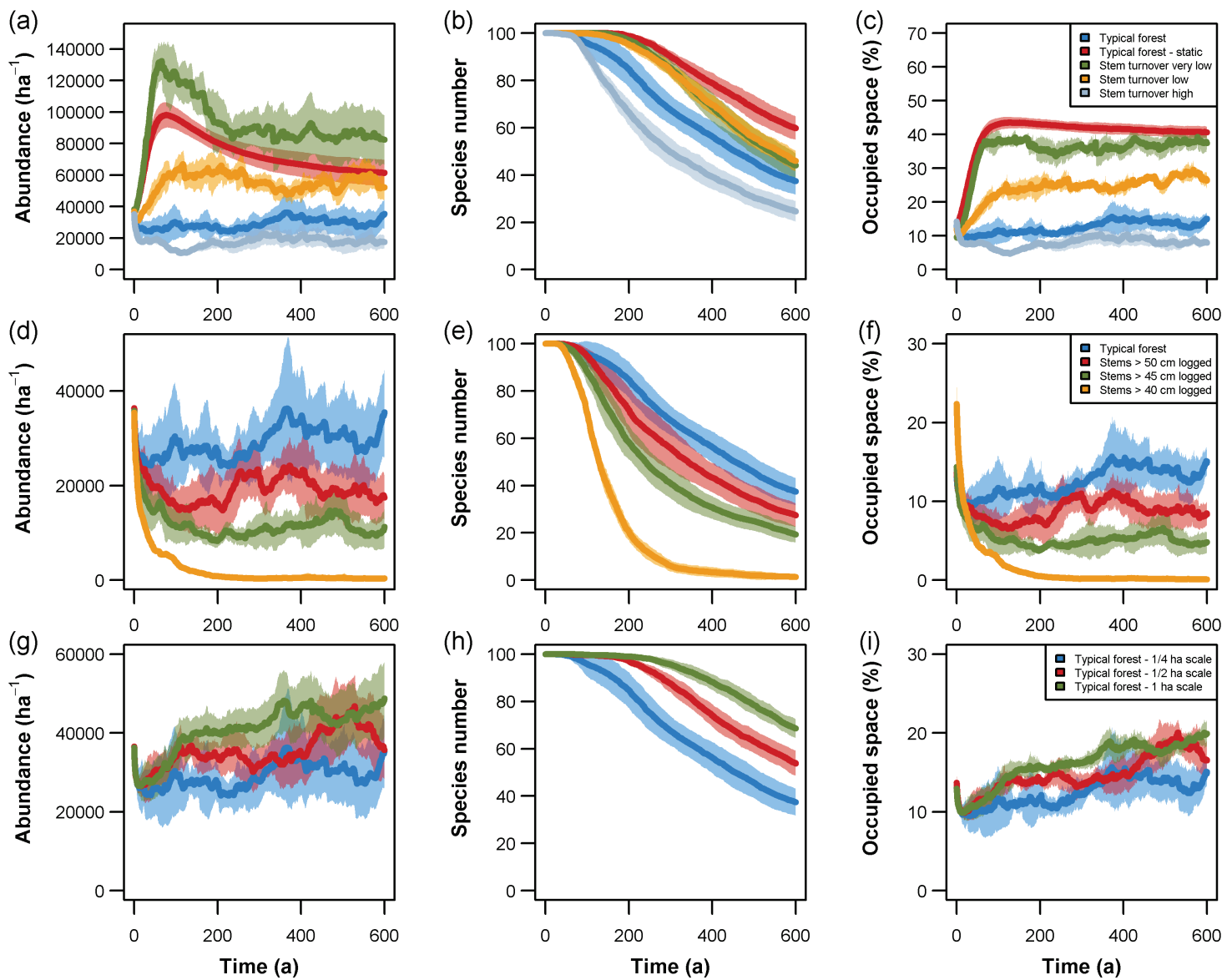

Figure 5.7. Effects of differences in forest dynamics, logging regimes and fragment sizes on abundances, species numbers and occupied substrate areas of epiphyte assemblages. Each panel shows the averaged temporal development of epiphyte assemblages over 600 years in forest stands with different forest dynamics, logging regimes and fragment size: (a-c) Forests differing in their natural dynamics (Fig. D.1), (d-f) forests differing in their logging intensity (Fig. D.2), and (g-i) forests differing in their fragment size (Fig. D.3). For each of these forest scenarios, five replicates were simulated and used as input data for the epiphyte model. In addition, ten different species pools of vascular epiphyte were generated and separately simulated for each forest replicate. Thus, for each forest scenarios a total of 50 epiphyte simulation were conducted, and mean values (bold lines) and standard deviations (shaded areas) are shown here.

\subsection{Discussion}

In this study, we coupled an individual-based epiphyte model with a functional-structural forest model to analyze how natural or human-induced differences in long-term forest dynamics affect the structure and dynamics of epiphyte assemblages. Before the simulation experiments were conducted, the model was validated with field data from two lowland sites in Panama and Ecuador. 


\section{Model validation}

Epiphyte assemblages simulated on typical lowland forests showed pronounced fluctuations in abundance in single model runs (Figs. 5.3 and D.4) but a comparably stable equilibrium when averaged over all model runs (Fig. 5.7a). Direct comparisons with observations are currently not possible as data on the community dynamics over such long periods are generally rare (e.g. Silvertown et al. 2006) and non-existent for epiphytes. Hence, validation of the dynamic model behavior can only be based on the few studies with repeated censuses covering shorter periods of time. In a lowland forest in Venezuela, an increase in abundance from 940 to 1516 individuals within four years was observed (1 ha plot; Schmidt-Neuerburg 2002) and a similar relative increase from $\sim 11,500$ to $>17,000$ individuals was found in a Panamanian forest ( 0.4 ha plot; first census: Zotz \& Schultz 2008; second census: G. Mendieta-Leiva, K. Wagner \& G. Zotz, unpublished data). In addition, Laube \& Zotz (2006) monitored changes of epiphyte assemblages on a palm tree species, and the total number of individuals increased from 763 (1999) to 899 (2002) and 957 (2004). These observed positive trends on the scale of individual trees and small study plots clearly hint to unsaturated epiphyte communities. We observe similar positive trends in community growth rates over even longer periods in our simulation models (Figs. D.4 and D.5). However, in our simulations such increasing trends were interrupted by pronounced tree fall events, which resulted in sharp decreases in abundances and prevented the epiphyte community from being saturated (compare dynamic and static lowland forest in Fig. 5.7c). Zotz \& Schultz (2008) reported that a single large tree hosted almost $15 \%$ of all epiphytes in their 0.4 ha plot; such trees disproportionately abundant with epiphytes will eventually die and this leads to pronounced local losses of epiphytes. The highly dynamic behavior of the simulated epiphyte assemblage at the local scale (simulated area: $0.25 \mathrm{ha}$ ) thus seems to agree with observations, although the magnitude of fluctuations and the frequency of drastic reductions in abundance following large gap creating events cannot yet be verified by observations.

Our knowledge on community-wide mortality rates of vascular epiphytes is limited. Epiphytes may die in situ, for instance, owing to herbivory or desiccation, or they may fall to the forest floor either off branches or attached to branches, where their longevity is generally very limited (Matelson et al. 1993). Whereas in our model we are able to trace the fate of each individual, this is complicated in the field due the difficult access to the canopy. Hietz (1997) used repeated photographs of branch sections in a montane forest in Mexico to monitor epiphyte assemblages, and reported average annual mortality rates of $\sim 16 \%$. Our simulated average mortality rate of $\sim 14 \%$ agrees surprisingly well with these observations. However, we want to emphasize that almost half of the deaths in our simulations were based on the size-dependent mortality rate we included to account for mechanism not explicitly simulated. Mortality rates of epiphytes commonly decline with size (Zotz \& Schmidt 2006), increased vulnerability to drought in smaller 


\section{Forest-epiphyte model}

size-classes being considered the main cause (Winkler, Hülber \& Hietz 2005; Zotz et al. 2005). The size-dependent mortality rate thus represents this ontogenetic shift in mortality which, however, does not emerge from model mechanisms and thus should be regarded as free model parameter. In contrast, the additional causes of mortality in our model result from epiphyte-forest and epiphyte-epiphyte interactions. Mortality due to competition was of minor importance $(<1 \%)$, which is in line with observations in forests with low epiphyte densities (Zotz \& Vollrath 2003). Mortality due to falling branches or trees $(\sim 3.6 \%)$ and due to the fall off branches $(\sim 2.4 \%)$ was more important. Sarmento Cabral et al. (2015) estimated annual mortality rate of $4 \%$ related to these two causes of mortality based on sampling of the forest floor for fallen epiphytes and comparisons with the known abundance in the canopy. However, they did not include fallen trees. In his study based on photographs, Hietz (1997) observed annual mortality rates related to branchfall of $\sim 7 \%$. A direct comparison with these studies is difficult, also because the mortality rates in our model varied substantially (Table D.1). However, the magnitude of the simulated mortality rates is within the range of direct observations. In addition, as the relative importance of the different causes of mortality generally agrees with expectation in epiphyte systems, the representation of mortality in our model seem to be plausible.

Species numbers showed a general decline over time in all model runs. This is partly a result of the experimental design. We assume a closed forest system with no dispersal from outside and consequently, species that are lost due to local extinctions cannot recolonize. A similar situation is virtually impossible in natural systems, as even remote forest fragments are to some extent linked to epiphyte source areas via long-distance dispersal. Non-fragmented old-growth forests are usually characterized by rather stable species numbers, or even increasing species numbers following forest succession (Benavides, Wolf \& Duivenvoorden 2006). While direct comparisons between simulated changes in species diversity and observations are thus not useful, comparisons between model runs can be valuable as they indicate differences in species loss rates driven by forest dynamics (see simulation experiments below).

In contrast to our relatively meager quantitative data on community dynamics, we do have adequate knowledge of how epiphyte assemblages are structured. Species abundances typically show a right-skewed distribution with a few dominant species and rather rare species (Benavides et al. 2005, 2011; Laube \& Zotz 2006). Our model reproduced a similar right-skewed distribution but the relative proportion of rare species was underestimated (Fig. 5.4a). This deviating pattern can be explained by the above-mentioned specific design of the simulation experiments, in which a small, closed system without dispersal and recolonization from outside is simulated. Under these conditions, rare species inevitably go extinct. Epiphyte species often show a patchy scattered distribution and even in larger areas many of them are locally rare (Küper et al. 2004). This indicates that large fragment sizes or sufficient connectivity to source areas are required to support 
the many potentially rare species and our closed and small-scale system is simply not well-suited to reproduces this situation. However, as the contribution of rare species to total abundance is negligible, the pattern in abundance and the saturation level of the community should not be strongly biased by this limitation.

The vertical stratification of epiphyte species and the vertical distribution of individuals in the assemblage is another remarkable feature of epiphyte communities (Zotz 2007; Krömer et al. 2007; Petter et al. 2016). The vertical stratification of epiphyte species in our model was similar to field observations, although the variability in height ranges was less pronounced (Fig. 5.5). This general simulated pattern is not surprising as the light niche of each species is not an emerging model property but rather an adjustable trait itself that is randomly selected based on user-defined ranges. This niche-based approach should be appropriate to approximate potential niches of many species, but it may be too simplistic for species with complex niche requirements, for instance trunk specialist like many Hymenophyllaceae (Krömer et al. 2007), which often only inhabit the lower trunk parts, i.e. have a very narrow height range (Fig. $5.5 \mathrm{c}$ ).

In contrast to the vertical stratification of species, the vertical distribution of individuals in the epiphyte assemblage is more interesting because it is an emergent property of the modelled processes. Starting from an initial vertical distribution that largely resembled the surface area of arboreal substrate (Fig. D.6), accumulations of individuals in higher canopy zones consistent with observations in the reference assemblages were observed (Figs. D.6 and 5.3b). Such accumulations also agree with tree-based observations of abundance peaks in the inner crowns of large trees (Johansson 1974), which are often explained by favorable water and light conditions in this zones (ter Steege \& Cornelissen 1989; Benzing 1990). However, Zotz \& Schultz (2008) speculated that such pattern might, at least partly, reflect spatial differences in available substrate area, which, however, are almost impossible to measure in natural systems. Although we cannot know with certainty that the vertical distribution of substrate area in our forest models mirrors reality, it nevertheless is remarkable that an unequivocal effect of substrate area on epiphyte abundance was not simulated in later time periods. Although not explicitly analyzed, we assume that enhanced connectivity between suitable substrate areas in the canopy zone facilitates dispersal and establishment and thus might explain the simulated pattern. Based on the result of independent studies analyzing the spatial community structure of epiphytes, which indicated that effective dispersal and colonization is predominantly a very local process (Zotz et al. 1999; Trapnell, Hamrick \& Nason 2004; Trapnell et al. 2013), we applied rather local dispersal kernels. Such local dispersal is more effective when available substrate is more homogenously distributed as in the canopy zone, and not as patchy as in the understory, where the distance between suitable stems is larger. As this local dispersal mechanism not only led to realistic vertical frequency 


\section{Forest-epiphyte model}

distributions, but also reproduced the delayed colonization of understory trees reasonably well (see example in Box 1), dispersal seems to be appropriately represented in our model.

Natural plant communities are usually size-structured (Muller-Landau et al. 2006b; West, Enquist \& Brown 2009) and the two reference epiphyte assemblages unequivocally showed the expected trend towards lower densities of larger size classes (Fig. 5.6). Although simulated correlations were weaker than in the reference systems, the general size structure was reasonably wellreproduced by the model. Variations in population density with size within natural communities can largely be explained by how individuals use resources as a function of their size (Enquist, Brown \& West 1998; Brown et al. 2004). In our model, mortality and age/size at maturity were defined as a function of size: mortality rates decrease with size while maturity ages increase. Space was the only resource the individuals competed for and, in addition, the maximum size of species in the initial species pool was randomly chosen on a log-normal scale. This means that smaller species were overrepresented in the species pool. Both the defined size-skewed species pool, as well as the size-dependent behavior of individuals emerging from our model approach thus contributed to the size pattern of the community. Our approach might omit many other sizedependent processes in natural communities; however, for the purpose of this study it appears to be appropriate.

In summary, despite the relatively simple demographic model describing growth, reproduction and mortality of individual epiphytes in a complex and dynamic 3D forest structure, composition and structure of the epiphyte assemblage was adequately simulated and the long-term dynamic model behavior seems to be reasonable. Thus, the level of detail of our model appears to be appropriate for addressing the main research questions with simulation experiments.

\section{Simulation experiments}

In the first simulation experiment, we assessed the influence of difference in natural forest dynamics (Fig. D.1) on the dynamics of epiphyte assemblages (Fig. 5.7a-c). Four forest systems with different stem turnover rates were coupled with the epiphyte model, whereby the stem turnover rates represented typically variations between $1 \%$ and $3 \%$ per year in tropical rainforest (Phillips 1996; Phillips et al. 2004b; Lewis et al. 2004b). However, it should be noted that due to the complex interaction in forests, such variations in turnover rates also affected additional attributes in our models such as the residence time of above-ground biomass or the total basal area (Fig. D.1). A striking result of this study is that the forest-epiphyte interactions generally not only prevented saturation of the epiphyte community, but also that the saturation level was controlled by the dynamic behavior of the forest (Fig. 5.7). When assessing these results one 
should bear in mind that our approach allows us to separate the endogenous epiphyte dynamics (determined by the species traits) from exogenous effect of forest dynamics. In contrast, such clear separation is commonly not possible in natural systems as differences in climatic conditions simultaneously affect the epiphyte dynamics and the forest dynamics. Ding et al. (2016) investigated the relative importance of direct and indirect effects on epiphyte species diversity and abundance along an elevational gradient in a tropical rain forest in China. They found that the direct effect of increasing humidity with elevation and the indirect effect of differences in climatic conditions via forest structure were of similar importance in explaining a mid-elevation peak in epiphyte abundance. This is an interesting result as differences in abundance or epiphyte biomass are commonly attributed mainly to the effect of humidity (Gehrig-Downie et al. 2011). Not only forest structures (e.g. stem number, basal area, canopy height) commonly show significant changes with elevation (Girardin et al. 2010, 2013; Asner et al. 2013a), but also forest dynamics, and stem turnover rates tend to decrease with elevation (Stephenson \& Van Mantgem 2005; Galbraith et al. 2013). Thus, changes in forest dynamics, and not only in structure, with elevation might actually be important factors contributing to the commonly observed higher abundance and saturation levels at higher elevations.

Species richness of epiphytes usually shows similar trends with elevation and mid-elevation peaks in epiphyte diversity have been reported (e.g. Küper et al. 2004; Krömer et al. 2005; Cardelus, Colwell \& Watkins 2006). As discussed above, due to the design of the simulation experiments the effect of forest dynamics on species richness can be evaluated by comparing local extinction rates. Increasing tree turnover rates led to higher extinction rates (Fig. 5.7b), which is not surprising as a more dynamic system imposes an additional risk particularly to slow-developing species. Consequently, an additional indirect effect of lower tree turnover rates at higher elevations contributing to species richness seems possible. However, our model also indicated that species might go extinct due to competitive exclusion in systems with high epiphyte densities as in montane cloud forests. Nevertheless, whether competitive exclusion actually plays an important role in epiphyte systems is still under debate (Zotz et al. 1999; Benavides et al. 2005; Flores-Palacios \& Garcia-Franco 2006).

Our model demonstrated possible effects of changes in natural forest dynamics on abundance and species richness in epiphyte assemblages. In recent decades, an alarming increase in tree turnover rates in tropical forests has been observed, which might be linked to anthropogenic climate change or elevated CO2 concentrations (Phillips 1996; Phillips et al. 2004b; Lewis et al. 2004b). Our results thus suggest that not only the direct effects of climate changes, but also the indirect effects via their impacts on forest dynamics may significantly influence the structure and dynamics of epiphytes assemblages. 


\section{Forest-epiphyte model}

In the second experiment, we simulated the effect of selective logging on epiphyte assemblages. The removal of large trees above a certain diameter class had a negative impact on abundance and richness; this is in accordance with previous findings (Padmawathe 2004). The epiphyte assemblage even collapsed if the minimum tree size for logging was too small (in our model: 40 $\mathrm{cm}$ in DBH; Fig. 5.7d-f). Our results thus emphasize the particular importance of large trees for epiphyte conservation. Large trees often host a large number of epiphytes individuals and species because they provide a relatively stable substrate over decades (Grubb et al. 1963; Zotz \& Schultz 2008; Hundera et al. 2013). It seems plausible that particularly susceptible epiphyte species with slow demographic rates require such stable habitats to reach maturity and maintain vital populations. Sustainable logging strategies are thus required in managed forest systems with regard to epiphyte diversity. Unfortunately, large trees are generally declining globally, not only due to direct removal, but also due to increased frequency of droughts, air pollution or as sideeffects of forest fragmentation (Laurance et al. 2000; Lindenmayer, Laurance \& Franklin 2012). This trend might thus pose and additional threat to epiphyte diversity.

In the last simulation experiment, we focused on implications of fragment size for epiphyte assemblages. Unsurprisingly, the rate of local species loss increased with decreasing fragment size, i.e. in larger fragments a more diverse epiphyte assemblage was maintained over a longer time span (Fig. 5.7h). This result agrees with observations of lower species richness of many organisms in smaller forest fragments (Turner 1996; Pardini et al. 2005; Martensen, Pimentel \& Metzger 2008). Edge effects or limited immigration from outside are often discussed as probable reason for this pattern (Turner 1996). These effects, however, do not play a role in our model when comparing the different scenarios. Here, the disproportionate effect of local disturbance caused by larger gap-creating tree fall events in smaller fragments simply increases the chance of an entire population to become extinct. In addition to other negative effects associated with forest fragmentation such as isolation or changes in microclimate (Flores-Palacios \& García-Franco 2007; Cascante-Marín et al. 2009), this effect might also be of importance in natural epiphyte system, particularly when fragments are small. However, as many epiphyte species often show a patchy distribution and occur in low abundances, the minimum fragment size to exclude this effect is probably higher than the 1 ha fragment used in this modelling study. In line with previous studies our results thus emphasize the importance of intact large forests for epiphyte conservation (Flores-Palacios \& García-Franco 2007; Hundera et al. 2013).

\section{Outlook}

Despite their ecological importance in tropical forest systems, the number of studies on vascular epiphytes is low compared to soil-rooted plants and the theoretical knowledge on structure and 
dynamics of epiphyte assemblages is limited (Mendieta-Leiva \& Zotz 2015). While the difficult access to the canopy and a lack of standardized field protocols are major obstacles in epiphyte ecology, Mendieta-Leiva \& Zotz (2015) also highlighted the lack of a conceptual framework hampering theoretical advances in epiphyte ecology. Mechanistic modelling studies as presented here can be useful tools to increase our understanding of processes structuring epiphyte communities. Mechanistic model can help to disentangle cause and effect in the highly complex epiphyte system, which in the field is often complicated. In addition, they can cover time intervals which may be relavant for epiphyte dynamics, but are not realisable in field studies.

In this model, we focused on analyzing the effect of natural and human-induced differences in forest dynamics on the dynamics of epiphyte assemblage, but our coupled model has the potential to addresss a variety of additional research questions. For instance, the effects of forest dynamics not only on abundance and diversity, but also on community structure (e.g. vertical distribution or size structure) or trait structure of the community could be tested. In additon, each tree in the forest plot can be regarded as independent entity and analyses may thus go beyond the community as a whole. Several forest plots may be linked via dispersal and questions related to metacommunities may be addressed. The role of host specificity in epiphyte communites is often discussed (see review by Wagner, Mendieta-Leiva \& Zotz 2015), and the presented model can serve as a starting point to assess if difference in tree size, age or structure, as well as spatial autocorrelation in a forest, may lead to patterns which mistakenly may be interpreted as host specificity. A selection of the mentioned potential future model application is presented in more detail in Box 1. 


\section{Box 1 - Potential future model applications}

\section{Example 1: Community trait composition}

It is generally assumed that the environmental conditions at a site drive optimal trait values. In an epiphyte system, structure and dynamics of the substrate can be regarded as important environmental factors. These factors may vary within forest stands (e.g. gradients in area and longevity of the substrate from lower trunks to outer branches) and among forest stands (e.g. differences in $\mathrm{AGB}$ and stem turnover rates). How trait distributions of epiphytes within stands are influenced by these factors, or how community-weighted trait means vary along forest dynamics gradients (see figure to the right) are thus interesting research topics and our trait-based model is well-suited for such studies.

\section{Example 2: Epiphyte communities on individual}

\section{trees}

Many factors influence the dynamics of epiphyte communities on individual trees. The position of the host tree in relation to surrounding epiphyte-harboring trees, as well as the change in tree structure during ontogeny plays an important role. As in natural forests important factors such as the total surface area or the age of a tree are usually not known, it is virtually impossible to determine their relative importance for the epiphyte assemblage. For instance, observed differences in epiphyte abundance on similar-sized trees might result from differences in tree age, however, this cannot be verified under field conditions. In contrast, our omniscient perspective in the model allows tracing the spatio-temporal of trees and their epiphyte community over all time steps (see figure to the right showing how diversity and abundance develops on a randomly selected canopy tree over the entire tree life span of $\sim 150$ years). Future modelling studies assessing the relative importance of factors potentially influencing epiphyte demography on individual trees can thus add to our understanding of the structure and dynamics of epiphyte assemblages.
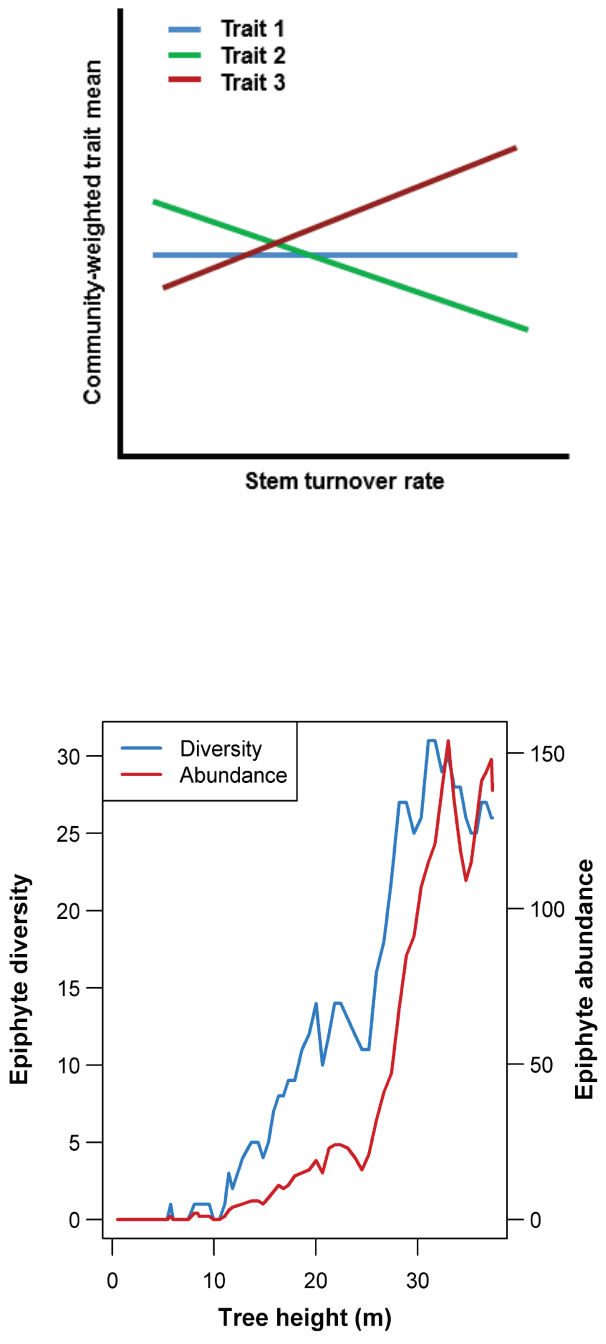


\section{Conclusion}

Many epiphyte communities show no signs of saturation and this modelling study has demonstrated that the average abundance/biomass of epiphytes in a forest stand can be strongly influenced by forest dynamics. While climatic variables such as annual precipitation or temperature are commonly reported in epiphyte studies, variables describing the structure (e.g. AGB, basal area, canopy height) or dynamics of the forest (stem turnover rates, residence time) are rarely reported. According to the results of this study, such metadata can be valuable and thus we propose to include them if possible.

Field data are essential to parameterize and validate ecological models. Long-term data of epiphyte assemblage are desirable, but we are fully aware that collecting such data is very laborintensive and time-consuming. Thus, we recommend that a particularly important aspect of community dynamics of epiphyte, i.e. the mortality due to branch, tree and forest dynamic, warrants more attention. Such studies can be conducted without climbing trees, for instance by systematic collections of fallen epiphytes on the forest floor. 



\section{Synopsis}

In chapter 2, I analyzed vertical gradients of ten leaf traits based on samples of $>1100$ individuals belonging to 83 epiphyte species. This study represents the most comprehensive study on vertical trait gradients of vascular epiphytes to date. As hypothesized, I observed that community mean trait values of many leaf traits were strongly correlated with height above ground. These results thus provide a more detailed picture of the community trait structure of epiphytes than previous studies focusing on comparing trait of epiphytes from pre-defined zones within trees (e.g. Andrade \& Nobel 1997; Hietz \& Briones 1998) or within the forest (e.g. Mantovani 1999; Stuntz $\&$ Zotz 2001). In addition, this suggests that height above ground is a suitable approximation of vertical environmental gradients and should be used in addition to frequently used zonation schemes in trait-based epiphyte studies. Both linear and non-linear trends were observed, and the non-linear decline in specific leaf area (SLA) indicates that light is probably the main driver of this trend (McMurtrie \& Dewar 2011). In contrast, the linear trend in SLA commonly observed in trees is often related to both light and hydraulic constraints (Rijkers et al. 2000; Kenzo et al. 2006). This example suggests ecophysiological differences between epiphyte and soil-rooted plant with regard to their trait response.

I found that intraspecific trait variability was pronounced and accounted for one-third of total observed trait variance, which is in the same range as observed for soil-rooted plants (Hulshof \& Swenson 2010; Albert et al. 2010a). Intraspecific trait adjustments along the vertical gradient were common and seventy per cent of all species showed significant trait-height relationships. Such trait adjustments were pronounced for some species, and individuals could have markedly different traits although separated by only few meters along the vertical gradient. Moreover, intraspecific trait variability was positively correlated with the vertical range occupied by species; however, this correlation was rather weak. This indicates that epiphyte species that can adjust their leaf traits to the environment can potentially occupy larger vertical ranges, but additional unexplored characteristics (e.g. root traits) may also play an important role and deserve attention in further studies.

I observed differences in leaf trait syndromes among taxonomic groups (orchids, bromeliads, aroids, ferns). Orchids, for instance, had on average the thickest leaves and lowest SLA values, while ferns were characterized by high leaf dry matter contents. These results are in line with previous findings (Hietz et al. 1999; Stuntz \& Zotz 2001; Cardelús \& Mack 2010). However, trait variability of species within the taxonomic groups was often pronounced, and between-group trait differences were often not significant between all groups but rather only between two groups. In the multidimensional trait space, the trait space occupied by these groups overlapped 


\section{Synopsis}

considerably. This shows that the epiphytic taxa do not form clearly distinguishable groups regarding their leaf traits, but the unique tendencies within taxonomic groups nevertheless indicate that some leaf traits are taxonomically conserved. I further demonstrated that leaf trait syndromes and the intraspecific trait variability play important roles in explaining the vertical zonation of vascular epiphyte species and taxonomic groups. However, other adaptations of epiphytes, such as water- and nutrient storing pseudobulbs in orchids or phytotelmata in bromeliads are probably likewise important.

In chapter 3, I demonstrated that the forest floor can be a rich source of information that has largely been neglected in epiphyte ecology. I found a considerable proportion of the epiphyte species occupying the forest canopies on the forest floor, either still attached to branches or fallen off branches. At the Brazilian site, the density of fallen epiphyte was higher $\left(\sim 3600 \mathrm{ha}^{-1}\right)$ than at the Panamanian site $\left(\sim 1100 \mathrm{ha}^{-1}\right)$. I estimated a mortality rate of at least $4 \%$ per year when considering the entire known community in Panama, and a mortality rate of $13 \%$ when considering epiphyte on branches $<10 \mathrm{~cm}$ in DBH. These results agree with previous studies (Hietz 1997) and underline the particular importance of tree and forest dynamics for the demography of vascular epiphytes.

Furthermore, trends in abundance, richness and composition over branch diameter reflected trends in the forest canopy. I argue that forest floor surveys provide useful floristic and, most notably, demographic information particularly on epiphytes occurring on the thinnest branches, which are least accessible with the most common techniques (e.g. rope-climbing, binoculars). Here, branchfall acts as an important demographic filter structuring epiphyte communities. My study thus indicates that, while epiphyte ecologists tend to look up, an occasional look down can also be worthwhile and may uncover unexplored source of ecological information about epiphytes.

In chapter 4, I developed a long-term dynamic forest stand model in which trees are represented by their three-dimensional structure. In previous forest models, trees were either represented by much simpler crown structures (Huth, Ditzer \& Bossel 1997; Liu 1998), or, when complex 3D structures were simulated, the forest models focused on even age-stands in single species systems over limited time frames (Feng et al. 2011; Guillemot et al. 2014). The novelty of my model is thus the combination of a high degree of detail with long-term demographic simulations. While rather complex at first sight, my model is based on relatively simple principles. Basically, lightdriven carbon assimilation and the within-tree carbon allocation are coupled using the principles of the pipe model theory (Shinozaki et al. 1964), whereby carbon assimilation is driven by leaf traits under consideration of between-trait trade-offs and correlations (Wright et al. 2004). In other words, leaf trait trade-offs were scaled to the whole-tree level, and this approach captured life 
history variation between different functional groups. For instance, species with high SLA values showed high initial growth rates, but had lower maximum heights and shorter life spans, i.e. characteristics that are attributed to pioneer species. Trait-based tree growth in my model is thus consistent with observations and supports the notion that the growth-survival trade-off of tropical tree species is, at least partly, determined by their leaf traits (Sterck et al. 2006). As tropical forest are generally very species-rich (Gentry 1988; ter Steege et al. 2013), functional groups aggregating tree species with similar growth characteristics are usually used in forest models, and parameters like growth rate or potential height are estimated based on empirical data for each group (e.g. Köhler \& Huth 1998; Tietjen \& Huth 2006). In my model, such characteristics emerge from the leaf investment strategy and are not defined a priori; it thus helps to understand the bottom-up mechanisms regulating tree growth.

In addition, between-tree competition and demographic processes (establishment, mortality) were integrated at the stand scale. The simulated forests reached dynamic equilibrium states in terms of above-ground biomass and stem number after 80-100 years, which lies well within the reported range (e.g. Fearnside \& Guimarães 1996; Hughes, Kauffman \& Jaramillo 1999). In this equilibrium important forest attributes were within observed ranges of typical Neotropical lowland forests (e.g. Malhi et al. 2006; Quesada et al. 2012). Moreover, detailed patterns such as the vertical leaf area density were also reproduced. This indicates that a structurally-realistic forest can be simulated with my model. As a consequence, the ability of my approach to describe multiple physiognomic and structural patterns as well as the dynamics of these patterns at multiple scales (from within-tree up to whole forest stand distribution of tree elements) provides multiple opportunities for model validation (Grimm et al. 2005). This is an important model property, as the model complexity is accompanied by a high number of emergent patterns which can be crosschecked against real-world data. The general principles applied in my approach also provide generalizable results (Evans et al. 2013), while retaining the possibility to be calibrated to generate the patterns of specific systems, as exemplified in chapter 4 for Neotropical lowland forests. In this sense, the development and validation of the model was the main focus in this chapter, but considering future studies, it has the potential to address pending general questions in tree and forest ecology as well as questions that might be specific for particular systems. For instance, the effects of frequent disturbances, logging, or changing environmental conditions on forest stability (dynamics equilibrium) and structure could be analyzed in detail with my model.

In chapter 5, I presented the first mechanistic model developed for vascular epiphytes. I coupled the forest stand model with an individual-based epiphyte model and assessed how differences in natural forest dynamics, selective logging and the size of the forest stand influenced the long-term dynamics of epiphyte assemblages. At first, emerging patterns were analyzed to validate the model. Starting from an even initial distribution (100 individuals per species), a right-skewed 


\section{Synopsis}

rank-abundance distributions with a few dominant species and many rather rare species was simulated, which is consistent with observations in natural epiphytic and non-epiphytic plant communities (Benavides et al. 2005, 2011; Laube \& Zotz 2006). In addition, the simulated assemblage was clearly size-structured and dominated by smaller individuals. This agrees with field observations of natural communities - an explanation being size-dependent resource use (Enquist, Brown \& West 1998; Brown et al. 2004). When averaged over multiple simulated replicates, epiphyte abundance reached a relatively stable equilibrium, but in single model runs pronounced fluctuations were observed. This means that epiphyte abundance tended to increase over time. However, such increasing trends were interrupted by pronounced tree fall events, which resulted in sharp decreases in abundances and prevented the epiphyte community from becoming saturated. Therefore, the observed trends of increasing abundances in the available data for vascular epiphytes (Schmit-Neuerburg 2002; Zotz \& Schultz 2008) could be explained by the lack of tree fall or gap formation within the time frame and spatial extent of these studies. This indicated the importance of tree turnover for epiphyte communities, and in subsequent simulation experiments, the effects of differences in natural forest dynamics were analyzed.

Tree turnover rates typically vary from 1\% to 3\% per year in tropical rainforest (Phillips 1996; Phillips et al. 2004b; Lewis et al. 2004b), and such variations had a marked impact on epiphyte diversity, abundance and 'saturation level' of epiphyte communities. Due to size-differences, abundance as such is ambiguous, and the percentage of arboreal substrate area occupied by epiphytes was thus used as an approximation of the saturation level of the epiphyte community. Even in mature lowland forests, epiphyte communities typically show no signs of saturation (Schmit-Neuerburg 2002; Laube \& Zotz 2006), and my model demonstrated that the saturation level is related to forest dynamics. The advantage of my modelling approach is that it allows separating the endogenous epiphyte dynamics (determined by the species traits) from the exogenous effects of forest dynamics. In contrast, such clear separation is commonly not possible in natural systems as differences in climatic conditions simultaneously affect the epiphyte dynamics and the forest dynamics. However, a recent study by Ding et al. (2016) applied structural equation models to disentangle the direct effects of climate and soil on epiphyte diversity and abundance along an elevational gradient from the indirect effects via forest structure. Interestingly, indirect effects explained a similar proportion of variations in abundance and species richness as humidity. In line with this study, my modelling approach thus highlights the importance of forest dynamics for epiphyte dynamics.

Furthermore, a decrease in tree size selected for exclusion, effectively mimicking an increased intensity of selective logging, as well as decreasing fragment sizes had the expected negative influences on epiphyte diversity. These observations thus additionally emphasize the particular values of undisturbed primary forests for biodiversity conservation (Barlow et al. 2007; Gibson 
et al. 2011). In fact, all three simulation experiments presented in this chapter represented human impacts such as i) environmental change (e.g. intensified forest dynamics via global warming), ii) selective logging and iii) habitat fragmentation. Therefore, experiments such as these can provide important information for epiphyte conservation. For example, future studies with my model can identify which functional types of epiphytes first disappear with increasing dynamics, decreasing tree size for logging and decreasing forest fragment size. This information can help to prioritize conservation efforts, while also helping to develop mitigation strategies to reverse loss of functional diversity. Therefore, the results of chapter 5 demonstrate that mechanistic models can be valuable tools to increase our understanding of the dynamics of epiphyte communities and to provide useful feedbacks to both empirical studies and conservation policies. Here, the coupled forest-epiphyte model can be regarded as virtual laboratory allowing us to address many more research questions regarding vascular epiphytes in the future.

In summary, the findings of my research improved our understanding of how the forest structure and dynamics affects the (trait) structure and dynamics of epiphyte communities. My thesis constitutes the most comprehensive study on the community trait structure of vascular epiphytes to date and, moreover, introduced mechanistic models to the field of epiphyte ecology. These modelling approaches open new avenues for future studies of spatial and temporal dynamics of vascular epiphyte assemblages while integrating epiphyte research in a more theoretical context. 


\section{References}

Ackerly, D.D. \& Cornwell, W.K. (2007) A trait-based approach to community assembly: partitioning of species trait values into within- and among-community components. Ecology letters, 10, 135-45.

Addicott, F. (1991) Abscission: shedding of parts. Physiology of trees, Wiley (ed A. Raghavendra), pp. 273-300. New York.

Adekunle, V.A.J., Olagoke, A.O. \& Akindele, S.O. (2013) Tree species diversity and structure of a Nigerian strict nature reserve. Tropical Ecology, 54, 275-289.

Albert, C.H., de Bello, F., Boulangeat, I., Pellet, G., Lavorel, S. \& Thuiller, W. (2012) On the importance of intraspecific variability for the quantification of functional diversity. Oikos, 121, 116-126.

Albert, C.H., Thuiller, W., Yoccoz, N.G., Douzet, R., Aubert, S. \& Lavorel, S. (2010a) A multi-trait approach reveals the structure and the relative importance of intra- vs. interspecific variability in plant traits. Functional Ecology, 24, 1192-1201.

Albert, C.H., Thuiller, W., Yoccoz, N.G., Soudant, A., Boucher, F., Saccone, P. \& Lavorel, S. (2010b) Intraspecific functional variability: extent, structure and sources of variation. Journal of Ecology, 98, 604-613.

Allen, M.T., Prusinkiewicz, P. \& DeJong, T.M. (2005) Using L-systems for modeling source-sink interactions, architecture and physiology of growing trees: the L-PEACH model. The New Phytologist, 166, 869-80.

Alves, L.F. \& Santos, F.A.M. (2002) Tree allometry and crown shape of four tree species in Atlantic rain forest, southeast Brazil. Journal of Tropical Ecology, 18, 245-260.

Anderson, M.J. (2001) A new method for non-parametric multivariate analysis of variance. Austral Ecology, 26, 3246.

Andrade, J.L. \& Nobel, P.S. (1997) Microhabitats and water relations of epiphytic cacti and ferns in a lowland Neotropical forest. Biotropica, 29, 261-270.

Anten, N.P.R. \& Schieving, F. (2010) The role of wood mass density and mechanical constraints in the economy of tree architecture. The American Naturalist, 175, 250-260.

Aragão, L., Malhi, Y., Metcalfe, D.B., Silva-Espejo, J.E., Jiménez, E., Navarrete, D., Almeida, S. ,Costa, A.C.L., Salinas, N., Phillips, O.L., Anderson, L.O., Alvarez, E., Baker, T.R., Goncalvez, P.H., Huamán-Ovalle, J., Mamani-Solórzano, M., Meir, P., Monteagudo, A., Patiño, S., Peñuela, M.C., Prieto, A., Quesada, C.A., Rozas-Dávila, A., Rudas, A., Silva Jr., J.A. \& Vásquez, R. (2009) Above-and below-ground net primary productivity across ten Amazonian forests on contrasting soils. Biogeoscience, 6, 2759-2778.

Asner, G.P., Anderson, C., Martin, R.E., Knapp, D.E., Tupayachi, R., Kennedy-Bowdoin, T., Sinca, F. \& Malhi, Y. (2013a) Landscape-scale changes in forest structure and functional traits along an Andes-to-Amazon elevation gradient. Biogeosciences Discussions, 10, 15415-15454.

Asner, G.P., Kellner, J.R., Kennedy-Bowdoin, T., Knapp, D.E., Anderson, C. \& Martin, R.E. (2013b) Forest canopy gap distributions in the southern Peruvian Amazon. PloS one, 8, e60875.

Asner, G.P., Nepstad, D., Cardinot, G. \& Ray, D. (2004) Drought stress and carbon uptake in an Amazon forest measured with spaceborne imaging spectroscopy. Proceedings of the National Academy of Sciences of the United States of America, 101, 6039-6044. 
Baker, T.R., Phillips, O.L., Malhi, Y., Almeidas, S., Arroya, L., Di Fiore, A., Erwin, T., Killeen, T., Laurance, S.G., Laurance, W.F., Lewis, S.L., Lloyd, J., Monteagudo, A., Neil, D.A., Patiño, S., Pitman, N.C.A., Silva, J.N.M. \& Martinez, R.V. (2004) Variation in wood density determines spatial patterns in Amazonian forest biomass. Global Change Biology, 10, 545-562.

Banin, L., Feldpausch, T.R., Phillips, O.L., Baker, T.R., Lloyd, J., Affum-Baffoe, K., Arets, E.J.M.M., Berry, N.J., Bradford, M., Brienen, R.J.W., Davies, S., Drescher, M., Higuchi, N., Hilbert, D.W., Hladik, A., Iida, Y., Salim, K.A., Kassim, A.R., King, D.A., Lopez-Gonzalez, G., Metcalfe, D., Nilus, R., Peh, K.S.H., Reitsma, J.M., Sonké, B., Taedoumg, H., Tan, S., White, L., Wöll, H. \& Lewis, S.L. (2012) What controls tropical forest architecture? Testing environmental, structural and floristic drivers. Global Ecology and Biogeography, 21, 1179-1190.

Banin, L., Lewis, S.L., Lopez-Gonzalez, G., Baker, T.R., Quesada, C.A., Chao, K.-J., Burslem, D.F.R.P., Nilus, R., Abu Salim, K., Keeling, H.C., Tan, S., Davies, S.J., Monteagudo Mendoza, A., Vásquez, R., Lloyd, J., Neill, D.A., Pitman, N. \& Phillips, O.L. (2014) Tropical forest wood production: a cross-continental comparison. Journal of Ecology, 102, 1025-1037.

Barczi, J.-F., Rey, H., Caraglio, Y., de Reffye, P., Barthélémy, D., Dong, Q.X. \& Fourcaud, T. (2008) AmapSim: a structural whole-plant simulator based on botanical knowledge and designed to host external functional models. Annals of Botany, 101, 1125-38.

Barlow, J., Gardner, T.A, Araujo, I.S., Avila-Pires, T.C., Bonaldo, A.B., Costa, J.E., Esposito, M.C., Ferreira, L.V., Hawes, J., Hernandez, M.I.M., Hoogmoed, M.S., Leite, R.N., Lo-Man-Hung, N.F., Malcolm, J.R., Martins, M.B., Mestre, L.A.M., Miranda-Santos, R., Nunes-Gutjahr, A.L., Overal, W.L., Parry, L., Peters, S.L., Ribeiro-Junior, M.A., da Silva, M.N.F., da Silva Motta, C. \& Peres, C.A. (2007) Quantifying the biodiversity value of tropical primary, secondary, and plantation forests. Proceedings of the National Academy of Sciences of the United States of America, 104, 18555-18560.

Barry, S.C. \& Welsh, A.H. (2002) Generalized additive modelling and zero inflated count data. Ecological Modelling, 157, 179-188.

Barthélémy, D. \& Caraglio, Y. (2007) Plant architecture: a dynamic, multilevel and comprehensive approach to plant form, structure and ontogeny. Annals of Botany, 99, 375-407.

de Bello, F., Lavorel, S., Albert, C.H., Thuiller, W., Grigulis, K., Dolezal, J., Janeček, Š. \& Lepš, J. (2011) Quantifying the relevance of intraspecific trait variability for functional diversity. Methods in Ecology and Evolution, 2, $163-174$.

Benavides, A.M., Duque, A.J., Duivenvoorden, J.F., Vasco, G.A. \& Callejas, R. (2005) A First quantitative census of vascular epiphytes in rain forests of Colombian Amazonia. Biodiversity and Conservation, 14, 739-758.

Benavides, A.M., Vasco, A., Duque, A.J. \& Duivenvoorden, J.F. (2011) Association of vascular epiphytes with landscape units and phorophytes in humid lowland forests of Colombian Amazonia. Journal of Tropical Ecology, 27, 223-237.

Benavides, A.M., Wolf, J.H.D. \& Duivenvoorden, J.F. (2006) Recovery and succession of epiphytes in upper Amazonian fallows. Journal of Tropical Ecology, 22, 705.

Benzing, D.H. (1990) Vascular Epiphytes. General biology and related biota. Cambridge University Press, Cambridge.

Berry, S.C., Varney, G.T. \& Flanagan, L.B. (1997) Leaf $\delta^{13} \mathrm{C}$ in Pinus resinosa trees and understory plants: variation associated with light and $\mathrm{CO}_{2}$ gradients. Oecologia, 109, 499-506.

Bertram, J.E.A. (1989) Size-dependent differential scaling in branches: the mechanical design of trees revisited. Trees, 4, 241-253. 


\section{References}

Bolnick, D.I., Amarasekare, P., Araújo, M.S., Bürger, R., Levine, J.M., Novak, M., Rudolf, V.H.W., Schreiber, S.J., Urban, M.C. \& Vasseur, D. (2011) Why intraspecific trait variation matters in community ecology. Trends in Ecology \& Evolution, 26, 183-92.

Bowman, D.M.J.S., Brienen, R.J.W., Gloor, E., Phillips, O.L. \& Prior, L.D. (2013) Detecting trends in tree growth: not so simple. Trends in Plant Science, 18, 11-17.

Bradford, M.G., Murphy, H.T., Ford, A.J., Hogan, D.L. \& Metcalfe, D.J. (2014) Long-term stem inventory data from tropical rain forest plots in Australia. Ecology, 95, 2362.

Brokaw, N.V.L. (1985) Gap-phase regeneration in a tropical forest. Ecology, 66, 682-687.

Brown, J.H., Gillooly, J.F., Allen, A.P., Savage, V.M. \& West, G.B. (2004) Toward a metabolic theory of ecology. Ecology, 85, 1771-1789.

Bugmann, H. (2001) A review of forest gap models. Climatic Change, 51, 259-305.

Burnham, K.P. \& Anderson, D.R. (2004) Multimodel inference: Understanding AIC and BIC in model selection. Sociological Methods \& Research, 33, 261-304.

Burns, K.C. \& Dawson, J. (2005) Patterns in the diversity and distribution of epiphytes and vines in a New Zealand forest. Austral Ecology, 30, 883-891.

Caldararu, S., Palmer, P.I. \& Purves, D.W. (2012) Inferring Amazon leaf demography from satellite observations of leaf area index. Biogeosciences, 9, 1389-1405.

Calvo-Alvarado, J.C., McDowell, N.G. \& Waring, R.H. (2008) Allometric relationships predicting foliar biomass and leaf area:sapwood area ratio from tree height in five Costa Rican rain forest species. Tree Physiology, 28, $1601-1608$.

Campbell, D.G., Daly, D.C., Prance, G.T. \& Maciel, U.N. (1986) Quantitative ecological inventory of terra firme and várzea tropical forest on the Rio Xingu, Brazilian Amazon. Brittonia, 38, 369-393.

Cannell, M.G.R. \& Thornley, J.H.M. (2000) Modelling the components of plant respiration: some guiding principles. Annals of Botany, 85, 45-54.

Cardelús, C.L. (2007) Vascular epiphyte communities in the inner-crown of Hyeronima alchorneoides and Lecythis ampla at La Selva Biological Station, Costa Rica. Biotropica, 39, 171-176.

Cardelús, C.L. \& Chazdon, R.L. (2005) Inner-crown microenvironments of two emergent tree species in a lowland wet forest. Biotropica, 37, 238-244.

Cardelús, C.L., Colwell, R.K. \& Watkins, J.E. (2006) Vascular epiphyte distribution patterns: explaining the midelevation richness peak. Journal of Ecology, 94, 144-156.

Cardelús, C.L. \& Mack, M.C. (2010) The nutrient status of epiphytes and their host trees along an elevational gradient in Costa Rica. Plant Ecology, 207, 25-37.

Cascante-Marín, A., von Meijenfeldt, N., de Leeuw, H.M.H., Wolf, J.H.D., Oostermeijer, J.G.B. \& den Nijs, J.C.M. (2009) Dispersal limitation in epiphytic bromeliad communities in a Costa Rican fragmented montane landscape. Journal of Tropical Ecology, 25, 63-73.

Catchpole, D.J. \& Kirkpatrick, J.B. (2010) The outstandingly speciose epiphytic flora of a single stranger fig (Ficus crassiuscula) in a Peruvian montane cloud forest. Tropical montane cloud forests (eds L.A. Bruijnzee, F.N. Scatena \& L.S. Hamilton), pp. 142-146. Cambridge University Press, New York.

Cavaleri, M.A., Oberbauer, S.F., Clark, D.B., Clark, D.A. \& Ryan, M.G. (2010) Height is more important than light in determining leaf morphology in a tropical forest. Ecology, 91, 1730-1739. 
Chambers, J.Q., Negron-Juarez, R.I., Marra, D.M., Di Vittorio, A., Tews, J., Roberts, D., Ribeiro, G.H.P.M., Trumbore, S.E. \& Higuchi, N. (2013) The steady-state mosaic of disturbance and succession across an old-growth Central Amazon forest landscape. Proceedings of the National Academy of Sciences of the United States of America, 110, 3949-3954.

Chambers, J.Q., dos Santos, J., Ribeiro, R.J. \& Higuchi, N. (2001) Tree damage, allometric relationships, and aboveground net primary production in central Amazon forest. Forest Ecology and Management, 152, 73-84.

Chao, K.-J., Phillips, O.L., Gloor, E., Monteagudo, A., Torres-Lezama, A. \& Martínez, R.V. (2008) Growth and wood density predict tree mortality in Amazon forests. Journal of Ecology, 96, 281-292.

Chase, M.W. (1986) Extreme twig epiphytism in the Oncidioid orchids. American Journal of Botany, 73, 756-757.

Chase, M.W. (1987) Obligate twig epiphytism in the Oncidiinae and other Neotropical orchids. Selbyana, 10, 24-30.

Chave, J., Condit, R., Lao, S., Caspersen, J.P., Foster, R.B. \& Hubbell, S.P. (2003) Spatial and temporal variation of biomass in a tropical forest: results from a large census plot in Panama. Journal of Ecology, 91, 240-252.

Chave, J., Navarrete, D., Almeida, S., Álvarez, E., Aragão, L.E.O.C., Bonal, D., Châtelet, P., Silva-Espejo, J.E., Goret, J.-Y., von Hildebrand, P., Jiménez, E., Patiño, S., Peñuela, M.C., Phillips, O.L., Stevenson, P. \& Malhi, Y. (2010) Regional and seasonal patterns of litterfall in tropical South America. Biogeosciences, 7, 43-55.

Chazdon, R.L. \& Fetcher, N. (1984) Photosynthetic light environments in a lowland tropical rain forest in Costa Rica. Journal of Ecology, 72, 553-564.

Chazdon, R.L, Finegan, B., Capers, R.S., Salgado-Negret, B., Casanoves, F., Boukili, V. \& Norden, N. (2010) Composition and dynamics of functional groups of trees during tropical forest succession in northeastern Costa Rica. Biotropica, 42, 31-40.

Clark, D.A. \& Clark, D.B. (1999) Assessing the growth of tropical rain forest trees: issues for forest modeling and management. Ecological Applications, 9, 981-997.

Clark, D.A. \& Clark, D.B. (2001) Getting to the canopy: tree height growth in a Neotropical rain forest. Ecology, 82, $1460-1472$.

Cline, M.G. (1997) Concepts and terminology of apical dominance. American Journal of Botany, 84, 1064-1069.

Coley, P.D. (1983) Herbivory and defensive characteristics of tree species in a lowland tropical forest. Ecological Monographs, 53, 209-233.

Condit, R. (1995) Research in large, long-term tropical forest plots. Trends in Ecology \& Evolution, 10, 18-22.

Cornelissen, J.H.C., Lavorel, S., Garnier, E., Díaz, S., Buchmann, N., Gurvich, D.E., Reich, P.B., ter Steege, H., Morgan, H.D., van der Hejden, M.G.A., Pausas, J.G. \& Poorter, H. (2003) A handbook of protocols for standardised and easy measurement of plant functional traits worldwide. Australian Journal of Botany, 51, $335-380$.

Cornwell, W.K. \& Ackerly, D.D. (2009) Community assembly and shifts in plant trait distributions across an environmental gradient in coastal California. Ecological Monographs, 79, 109-126.

Coste, S., Baraloto, C., Leroy, C., Marcon, E., Renaud, A., Richardson, A.D., Roggy, J.C., Schimann, H., Uddling, J. \& Herault, B. (2010) Assessing foliar chlorophyll contents with the SPAD-502 chlorophyll meter: a calibration test with thirteen tree species of tropical rainforest in French Guiana. Annals of Forest Science, 67, 607 .

Cramer, W., Bondeau, A., Woodward, F.I., Prentice, I.C., Betts, R.A., Brovkin, V., Cox, P.M., Fisher, V., Foley, J.A., Friend, A.D., Kucharik, C., Lomas, M.R., Ramankutty, N., Sitch, S., Smith, B., White, A. \& Young-Molling, 


\section{References}

C. (2001) Global response of terrestrial ecosystem structure and function to $\mathrm{CO}_{2}$ and climate change: results from six dynamic global vegetation models. Global Change Biology, 7, 357-373.

Davies, S.J. (2001) Tree mortality and growth in 11sympatric Macaranga species in Borneo. Ecology, 82, 920-932.

DeJong, T.M., Da Silva, D., Vos, J. \& Escobar-Gutierrez, A.J. (2011) Using functional-structural plant models to study, understand and integrate plant development and ecophysiology. Annals of Botany, 108, 987-989.

Denslow, J.S. (1987) Tropical rainforest gaps and tree species diversity. Annual Review of Ecology and Systematics, 18, 431-451.

DeWalt, S.J. \& Chave, J. (2004) Structure and biomass of four lowland Neotropical forests. Biotropica, 36, 7-19.

Díaz, S., Cabido, M. \& Casanoves, F. (1998) Plant functional traits and environmental filters at a regional scale. Journal of Vegetation Science, 9, 113-122.

Díaz, S., Hodgson, J.G., Thompson, K., Cabido, M., Cornelissen, J.H.C., Jalili, A., Montserrat-Martí, G., Grime, J.P., Zarrinkamar, F., Asri, Y., Band, S.R., Basconcelo, S., Castro-Díez, P., Funes, G., Hamzehee, B., Khoshnevi, M., Pérez-Harguindeguy, N., Pérez-Rontomé, M.C., Shirvany, F.A., Vendramini, F., Yazdani, S., AbbasAzimi, R., Bogaard, A., Boustani, S., Charles, M., Dehghan, M., de Torres-Espuny, L., Falczuk, V., Guerrero-Campo, J., Hynd, A., Jones, G., Kowsary, E., Kazemi-Saeed, F., Maestro-Martínez, M., RomoDíez, A., Shaw, S., Siavash, B., Villar-Salvador, P. \& Zak, M.R. (2004) The plant traits that drive ecosystems: Evidence from three continents. Journal of Vegetation Science, 15, 295-304.

Ding, Y., Liu, G., Zang, R., Zhang, J., Lu, X. \& Huang, J. (2016) Distribution of vascular epiphytes along a tropical elevational gradient: disentangling abiotic and biotic determinants. Scientific Reports, 6, 19706.

Dirzo, R. \& Raven, P.H. (2003) Global state of biodiversity and loss. Annual Review of Environment and Resources, 28, 137-167.

Domingues, T.F., Martinelli, L.A. \& Ehleringer, J.R. (2007) Ecophysiological traits of plant functional groups in forest and pasture ecosystems from eastern Amazônia, Brazil. Plant Ecology, 193, 101-112.

Doughty, C.E. \& Goulden, M.L. (2008) Seasonal patterns of tropical forest leaf area index and $\mathrm{CO}_{2}$ exchange. Journal of Geophysical Research, 113, G00B06.

Doughty, C.E., Metcalfe, D.B., da Costa, M.C., de Oliveira, A.A.R., Neto, G.F.C., Silva, J.A., Aragão, L.E.O.C., Almeida, S.S., Quesada, C.A., Girardin, C.A.J., Halladay, K., da Costa, A.C.L. \& Malhi, Y. (2014) The production, allocation and cycling of carbon in a forest on fertile terra preta soil in eastern Amazonia compared with a forest on adjacent infertile soil. Plant Ecology \& Diversity, 7, 41-53.

Dufrêne, M. \& Legendre, P. (1997) Species assemblages and indicator species: The need for a flexible asymmetrical approach. Ecological Monographs, 67, 345-366.

Duncan, R.P., Forsyth, D.M. \& Hone, J. (2007) Testing the metabolic theory of ecology: Allometric scaling exponents in mammals. Ecology, 88, 324-333.

Einzmann, H.J.R., Beyschlag, J., Hofhansl, F., Wanek, W. \& Zotz, G. (2015) Host tree phenology affects vascular epiphytes at the physiological, demographic and community level. AoB PLANTS, 7, plu073.

Elias, M. \& Potvin, C. (2003) Assessing inter- and intra-specific variation in trunk carbon concentration for 32 Neotropical tree species. Canadian Journal of Forest Research, 33, 1039-1045.

Enquist, B.J., Brown, J.H. \& West, G.B. (1998) Allometric scaling of plant energetics and population density. Nature, $395,163-165$. 
Enquist, B.J., West, G.B. \& Brown, J.H. (2009) Extensions and evaluations of a general quantitative theory of forest structure and dynamics. Proceedings of the National Academy of Sciences of the United States of America, 106, 7046-7051.

Erwin, T.L. (1988) The tropical forest canopy. Biodiversity, Wiley (ed E.O. Wilson), pp. 123-129. Washington

Evans, R.D. (2001) Physiological mechanisms influencing plant nitrogen isotope composition. Trends in Plant Science, 6, 121-126.

Evans, M.R., Bithell, M., Cornell, S.J., Dall, S.R.X., Díaz, S., Emmott, S., Ernande, B., Grimm, V., Hodgson, D.J., Lewis, S.L., Mace, G.M., Morecroft, M., Moustakas, A., Murphy, E., Newbold, T., Norris, K.J., Petchey, O., Smith, M., Travis, J.M.J. \& Benton, T.G. (2013) Predictive systems ecology. Proc R Soc B, 280, 20131452.

Evans, J.R. \& Poorter, H. (2001) Photosynthetic acclimation of plants to growth irradiance: the relative importance of specific leaf area and nitrogen partitioning in maximizing carbon gain. Plant, Cell \& Environment, 24, 755767.

Farnsworth, K.D. \& Niklas, K.J. (1995) Theories of optimization, form and function in branching architecture in plants. Functional Ecology, 9, 355-363.

Farquhar, G.D., Ehleringer, J.R. \& Hubick, K.T. (1989) Carbon isotope discrimination and photosynthesis. Annual Review of Plant Physiology, 40, 503-537.

Fearnside, P.M. \& Guimarães, W.M. (1996) Carbon uptake by secondary forests in Brazilian Amazonia. Forest Ecology and Management, 80, 35-46.

Feldpausch, T.R., Banin, L., Phillips, O.L., Baker, T.R., Lewis, S.L., Quesada, C.A.., Affum-Baffoe, K., Arets, E.J.M.M., Berry, N.J., Bird, M., Brondizio, E.S., de Camargo, P., Chave, J., Djagbletey, G., Domingues, T.F., Drescher, M., Fearnside, P.M., França, M.B., Fyllas, N.M., Lopez-Gonzalez, G., Hladik, A., Higuchi, N., Hunter, M.O., Iida, Y., Salim, K.A., Kassim, A.R., Keller, M., Kemp, J., King, D.A., Lovett, J.C., Marimon, B.S., Marimon-Junior, B.H., Lenza, E., Marshall, A.R., Metcalfe, D.J., Mitchard, E.T.A., Moran, E.F., Nelson, B.W., Nilus, R., Nogueira, E.M., Palace, M., Patiño, S., Peh, K.S.-H., Raventos, M.T., Reitsma, J.M., Saiz, G., Schrodt, F., Sonké, B., Taedoumg, H.E., Tan, S., White, L., Wöll, H. \& Lloyd, J. (2011) Heightdiameter allometry of tropical forest trees. Biogeosciences, 8, 1081-1106.

Feldpausch, T.R., Lloyd, J., Lewis, S.L, Brienen, R.J.W., Gloor, E., Montagudo Mendoza, A., Lopez-Gonzalez, G., Banin, L. et al. (2012) Tree height integrated into pan-tropical forest biomass estimates. Biogeosciences, 9 , $1-23$.

Feng, L., de Reffye, P., Dreyfus, P. \& Auclair, D. (2011) Connecting an architectural plant model to a forest stand dynamics model - application to Austrian black pine stand visualization. Annals of Forest Science, 69, 245255.

Fichtler, E., Clark, D.A. \& Worbes, M. (2003) Age and long-term growth of trees in an old-growth tropical rain forest, based on analyses of tree rings and ${ }^{14} \mathrm{C}$. Biotropica, 35, 306-317.

Flores-Palacios, A. \& Garcia-Franco, J.G. (2006) The relationship between tree size and epiphyte species richness: testing four different hypotheses. Journal of Biogeography, 33, 323-330.

Flores-Palacios, A. \& García-Franco, J.G. (2007) Habitat isolation changes the beta diversity of the vascular epiphyte community in lower montane forest, Veracruz, Mexico. Biodiversity and Conservation, 17, 191-207.

Foster, P. (2001) The potential negative impacts of global climate change on tropical montane cloud forests. EarthScience Reviews, 55, 73-106. 


\section{References}

Fourcaud, T., Zhang, X., Stokes, A., Lambers, H. \& Körner, C. (2008) Plant growth modelling and applications: the increasing importance of plant architecture in growth models. Annals of Botany, 101, 1053-1063.

Franklin, O., Johansson, J., Dewar, R.C., Dieckmann, U., McMurtrie, R.E., Brännström, A. \& Dybzinski, R. (2012) Modeling carbon allocation in trees: a search for principles. Tree Physiology, 32, 648-66.

Freiberg, M. (1996) Spatial distribution of vascular epiphytes on three emergent canopy trees in French Guiana. Biotropica, 28, 345-355.

Freiberg, M. \& Freiberg, E. (2000) Epiphyte diversity and biomass in the canopy of lowland and montane forests in Ecuador. Journal of Tropical Ecology, 16, 673-688.

Galbraith, D., Malhi, Y., Affum-Baffoe, K., Castanho, A.D.A.., Doughty, C.E., Fisher, R.A., Lewis, S.L., Peh, K.S.H., Phillips, O.L., Quesada, C.A., Sonké, B. \& Lloyd, J. (2013) Residence times of woody biomass in tropical forests. Plant Ecology \& Diversity, 6, 139-157.

Galbraith, D., Malhi, Y., Aragão, L. \& Baker, T. (2014) The ecosystem dynamics of Amazonian and Andean forests. Plant Ecology \& Diversity, 7, 1-6.

Gehrig-Downie, C., Obregón, A., Bendix, J. \& Gradstein, S.R. (2011) Epiphyte biomass and canopy microclimate in the tropical lowland cloud forest of French Guiana. Biotropica, 43, 591-596.

van Gelder, H.A., Poorter, L. \& Sterck, F.J. (2006) Wood mechanics, allometry, and life-history variation in a tropical rain forest tree community. New Phytologist, 171, 367-378.

Gentry, A.H. (1988) Tree species richness of upper Amazonian forests. Proceedings of the National Academy of Sciences of the United States of America, 85, 156-159.

Gentry, A.H. \& Dodson, C.H. (1987) Diversity and biogeography of Neotropical vascular epiphytes. Annals of the Missouri Botanical Garden, 74, 205-233.

Gibson, L., Lee, T.M., Koh, L.P., Brook, B.W., Gardner, T.A., Barlow, J., Peres, C.A., Bradshaw, C.J.A., Laurance, W.F., Lovejoy, T.E. \& Sodhi, N.S. (2011) Primary forests are irreplaceable for sustaining tropical biodiversity. Nature, 478, 378-381.

Girardin, C.A.J., Farfan-Rios, W., Garcia, K., Feeley, K.J., Jørgensen, P.M., Murakami, A.A., Cayola Pérez, L., Seidel, R., Paniagua, N., Fuentes Claros, A.F., Maldonado, C., Silman, M., Salinas, N., Reynel, C., Neill, D.A., Serrano, M., Caballero, C.J., La Torre Cuadros, M.D.L.A., Macía, M.J., Killeen, T.J. \& Malhi, Y. (2013) Spatial patterns of above-ground structure, biomass and composition in a network of six Andean elevation transects. Plant Ecology \& Diversity, 7, 1-11.

Girardin, C.A.J., Malhi, Y., Aragão, L.E.O.C., Mamani, M., Huaraca Huasco, W., Durand, L., Feeley, K.J., Rapp, J., Silva-Espejo, J.E., Silman, M., Salinas, N. \& Whittaker, R.J. (2010) Net primary productivity allocation and cycling of carbon along a tropical forest elevational transect in the Peruvian Andes. Global Change Biology, 16, 3176-3192.

Godin, C. \& Sinoquet, H. (2005) Functional-structural plant modelling. New Phytologist, 166, 705-708.

Gourlet-Fleury, S., Blanc, L., Picard, N., Sist, P., Dick, J., Nasi, R., Swaine, M.D. \& Forni, E. (2005) Grouping species for predicting mixed tropical forest dynamics: looking for a strategy. Annals of Forest Science, 62, 785-796.

Grassein, F., Till-Bottraud, I. \& Lavorel, S. (2010) Plant resource-use strategies: the importance of phenotypic plasticity in response to a productivity gradient for two subalpine species. Annals of Botany, 106, 637-645.

Grimm, V., Berger, U., Bastiansen, F., Eliassen, S., Ginot, V., Giske, J., Goss-Custard, J., Grand, T., Heinz, S.K., Huse, G. et al. (2006) A standard protocol for describing individual-based and agent-based models. Ecological Modelling, 198, 115-126. 
Grimm, V., Berger, U., DeAngelis, D.L., Polhill, J.G., Giske, J. \& Railsback, S.F. (2010) The ODD protocol: A review and first update. Ecological Modelling, 221, 2760-2768.

Grimm, V. \& Railsback, S.F. (2011) Pattern-oriented modelling: a "multi-scope" for predictive systems ecology. Philosophical Transactions of the Royal Society B: Biological Sciences, 367, 298-310.

Grimm, V., Revilla, E., Berger, U., Jeltsch, F., Mooij, W.M., Railsback, S.F., Thulke, H.-H., Weiner, J., Wiegand, T. \& DeAngelis, D.L. (2005) Pattern-oriented modeling of agent-based complex systems: lessons from ecology. Science, 310, 987-991.

Grubb, P.J., Lloyd, J.R., Pennington, T.D. \& Whitmore, T.C. (1963) A comparison of montane and lowland rain forest in Ecuador I. The forest structure, physiognomy, and floristics. Journal of Ecology, 51, 567-601.

Guillemot, J., Delpierre, N., Vallet, P., Francois, C., Martin-StPaul, N.K., Soudani, K., Nicolas, M., Badeau, V. \& Dufrene, E. (2014) Assessing the effects of management on forest growth across France: insights from a new functional-structural model. Annals of Botany, 114, 779-793.

Hacke, U.G., Sperry, J.S., Pockman, W.T., Davis, S.D. \& McCulloh, K.A. (2001) Trends in wood density and structure are linked to prevention of xylem implosion by negative pressure. Oecologia, 126, 457-461.

Hallé, F., Oldeman, R.A.A. \& Tomlinson, P.B. (1978) Tropical trees and forests: an architectural analysis. SpringerVerlag, Berlin, Heidelberg, New York.

Hassan, R., Scholes, R. \& Ash, N. (eds). (2005) Ecosystems and human well-being: current state and trends, Volume 1. Island Press, Washington DC.

Hector, A., Philipson, C., Saner, P., Chamagne, J., Dzulkifli, D., O’Brien, M., Snaddon, J.L., Ulok, P., Weilenmann, M., Reynolds, G. \& Godfray, H.C.J. (2011) The Sabah Biodiversity Experiment: a long-term test of the role of tree diversity in restoring tropical forest structure and functioning. Philosophical Transactions of the Royal Society B: Biological Sciences, 366, 3303-3315.

Helmer, E.H. \& Lefsky, M.A. (2006) Forest canopy heights in Amazon river basin forests as estimated with the Geoscience Laser Altimeter System (GLAS). USDA Forest Service Proceedings, RMRS-P-42CD, 802-808.

Hérault, B., Bachelot, B., Poorter, L., Rossi, V., Bongers, F., Chave, J., Paine, C.E.T., Wagner, F. \& Baraloto, C. (2011) Functional traits shape ontogenetic growth trajectories of rain forest tree species. Journal of Ecology, 99, $1431-1440$.

Herberich, E., Sikorski, J. \& Hothorn, T. (2010) A Robust Procedure for Comparing Multiple Means under Heteroscedasticity in Unbalanced Designs. PloS ONE, 5, e9788.

Heywood, V.H. \& Watson, R.T. (1995) Global Biodiversity Assessment. Cambridge University Press, New York.

Hietz, P. (1997) Population dynamics of epiphytes in a Mexican humid montane forest. Journal of Ecology, 85, 767775.

Hietz, P., Ausserer, J. \& Schindler, G. (2002) Growth, maturation and survival of epiphytic bromeliads in a Mexican humid montane forest. Journal of Tropical Ecology, 18, 177-191.

Hietz, P. \& Briones, O. (1998) Correlation between water relations and within-canopy distribution of epiphytic ferns in a Mexican cloud forest. Oecologia, 114, 305-316.

Hietz, P. \& Briones, O. (2001) Photosynthesis, chlorophyll fluorescence and within-canopy distribution of epiphytic ferns in a Mexican cloud forest. Plant Biology, 3, 279-287.

Hietz, P. \& Wanek, W. (2003) Size-dependent variation of carbon and nitrogen isotope abundances in epiphytic bromeliads. Plant Biology, 5, 137-142. 


\section{References}

Hietz, P., Wanek, W. \& Popp, M. (1999) Stable isotopic composition of carbon and nitrogen and nitrogen content in vascular epiphytes along an altitudinal transect. Plant, Cell and Environment, 22, 1435-1443.

Houghton, R.A., Lawrence, K.T., Hackler, J.L. \& Brown, S. (2001) The spatial distribution of forest biomass in the Brazilian Amazon: a comparison of estimates. Global Change Biology, 7, 731-746.

Hughes, R.F., Kauffman, J.B. \& Jaramillo, V.J. (1999) Biomass, carbon, and nutrient dynamics of secondary forests in a humid tropical region of México. Ecology, 80, 1892-1907.

Hulshof, C.M. \& Swenson, N.G. (2010) Variation in leaf functional trait values within and across individuals and species: an example from a Costa Rican dry forest. Functional Ecology, 24, 217-223.

Hundera, K., Aerts, R., De Beenhouwer, M., Van Overtveld, K., Helsen, K., Muys, B. \& Honnay, O. (2013) Both forest fragmentation and coffee cultivation negatively affect epiphytic orchid diversity in Ethiopian moist evergreen Afromontane forests. Biological Conservation, 159, 285-291.

Huth, A. \& Ditzer, T. (2000) Simulation of the growth of a lowland Dipterocarp rain forest with FORMIX3. Ecological Modelling, 134, 1-25.

Huth, A., Ditzer, T. \& Bossel, H. (1997) Rain forest growth model FORMIX3: A Tool for forest management planning towards sustainability. Deutsche Gesellschaft für Technische Zusammenarbeit (GTZ), Eschborn.

Iida, Y., Kohyama, T.S., Kubo, T., Kassim, A.R., Poorter, L., Sterck, F. \& Potts, M.D. (2011) Tree architecture and life-history strategies across 200 co-occurring tropical tree species. Functional Ecology, 25, 1260-1268.

Iida, Y., Poorter, L., Sterck, F.J., Kassim, A.R., Kubo, T., Potts, M.D. \& Kohyama, T.S. (2012) Wood density explains architectural differentiation across 145 co-occurring tropical tree species. Functional Ecology, 26, 274-282.

Iida, Y., Poorter, L., Sterck, F., Kassim, A.R., Potts, M.D., Kubo, T. \& Kohyama, T.S. (2014) Linking size-dependent growth and mortality with architectural traits across 145 co-occurring tropical tree species. Ecology, 95, 353363.

Johansson, D. (1974) Ecology of vascular epiphytes in West African rain forest. Acta Phytogeographica Suecia, 59, $1-136$.

Kattge, J., Díaz, S., Lavorel, S., Prentice, I.C., Leadley, P., Bönisch, G., Garnier, E., Westoby, M., Reich, P.B., Wright, I.J., Cornelissen, J.H.C., Violle, C., Harrison, S.P., Van Bodegom, P.M., Reichstein, M., Enquist, B.J., Soudzilovskaia, N.A., Ackerly, D.D., Anand, M., Atkin, O., Bahn, M., Baker, T.R., Baldocchi, D., Bekker, R., Blanco, C.C., Blonder, B., Bond, W.J., Bradstock, R., Bunker, D.E., Casanoves, F., Cavender-Bares, J., Chambers, J.Q., Chapin Iii, F.S., Chave, J., Coomes, D., Cornwell, W.K., Craine, J.M., Dobrin, B.H., Duarte, L., Durka, W., Elser, J., Esser, G., Estiarte, M., Fagan, W.F., Fang, J., Fernández-Méndez, F., Fidelis, A., Finegan, B., Flores, O., Ford, H., Frank, D., Freschet, G.T., Fyllas, N.M., Gallagher, R. V., Green, W.A., Gutierrez, A.G., Hickler, T., Higgins, S.I., Hodgson, J.G., Jalili, A., Jansen, S., Joly, C.A., Kerkhoff, A.J., Kirkup, D., Kitajima, K., Kleyer, M., Klotz, S., Knops, J.M.H., Kramer, K., Kühn, I., Kurokawa, H., Laughlin, D., Lee, T.D., Leishman, M., Lens, F., Lenz, T., Lewis, S.L., Lloyd, J., Llusià, J., Louault, F., Ma, S., Mahecha, M.D., Manning, P., Massad, T., Medlyn, B.E., Messier, J., Moles, A.T., Müller, S.C., Nadrowski, K., Naeem, S., Niinemets, Ü., Nöllert, S., Nüske, A., Ogaya, R., Oleksyn, J., Onipchenko, V.G., Onoda, Y., Ordoñez, J., Overbeck, G., Ozinga, W.A., Patiño, S., Paula, S., Pausas, J.G., Peñuelas, J., Phillips, O.L., Pillar, V., Poorter, H., Poorter, L., Poschlod, P., Prinzing, A., Proulx, R., Rammig, A., Reinsch, S., Reu, B., Sack, L., Salgado-Negret, B., Sardans, J., Shiodera, S., Shipley, B., Siefert, A., Sosinski, E., Soussana, J.F., Swaine, E., Swenson, N., Thompson, K., Thornton, P., Waldram, M., Weiher, E., White, M., White, S., Wright, S.J., Yguel, B., Zaehle, S., Zanne, A.E. \& Wirth, C. (2011) TRY - a global database of plant traits. Global Change Biology, 17, 2905-2935. 
Kelly, D.L., O’Donovan, G., Feehan, J., Murphy, S., Drangeid, S.O. \& Marcano-Berti, L. (2004) The epiphyte communities of a montane rain forest in the Andes of Venezuela: patterns in the distribution of the flora. Journal of Tropical Ecology, 20, 643-666.

Kenzo, T., Ichie, T., Watanabe, Y., Yoneda, R., Ninomiya, I. \& Koike, T. (2006) Changes in photosynthesis and leaf characteristics with tree height in five dipterocarp species in a tropical rain forest. Tree Physiology, 26, 865873.

Kichenin, E., Wardle, D.A., Peltzer, D.A., Morse, C.W. \& Freschet, G.T. (2013) Contrasting effects of plant inter- and intraspecific variation on community-level trait measures along an environmental gradient. Functional Ecology, 27, 1254-1261.

King, D.A. (1991) Correlations between biomass allocation, relative growth rate and light environment in tropical forest saplings. Functional Ecology, 5, 485-492.

King, D.A. (1996) Allometry and life history of tropical trees. Journal of Tropical Ecology, 12, 25-44.

King, D.A. (1997) Branch growth and biomass allocation in Abies amabilis saplings in contrasting light environments. Tree Physiology, 17, 251-258.

King, D.A., Leigh, E.G., Condit, R., Foster, R.B. \& Hubbell, S.P. (1997) Relationships between branch spacing, growth rate and light in tropical forest saplings. Functional Ecology, 11, 627-635.

Kitajima, K. (1994) Relative importance of photosynthetic traits and allocation patterns as correlates of seedling shade tolerance of 13 tropical trees. Oecologia, 98, 419-428.

Kitajima, K., Mulkey, S.S. \& Wright, S.J. (2005) Variation in crown light utilization characteristics among tropical canopy trees. Annals of Botany, 95, 535-547.

Kitching, R.L. (2006) Crafting the pieces of the diversity jigsaw puzzle. Science, 313, 1055-1057.

Kniemeyer, O.G.M. (2008) Design and implementation of a graph grammar based language for Functional-Structural Plant Modelling. PhD Thesis, University of Cottbus.

Kobe, R.K. (1999) Light gradient partitioning among tropical tree species through differential seedling mortality and growth. Ecology, 80, 187-201.

Koch, G.W., Sillett, S.C., Jennings, G.M. \& Davis, S.D. (2004) The limits to tree height. Nature, 428, 851-854.

Köhler, P. \& Huth, A. (1998) The effects of tree species grouping in tropical rainforest modelling: Simulations with the individual-based model FORMIND. Ecological Modelling, 109, 301-321.

Kreft, H., Köster, N., Küper, W., Nieder, J. \& Barthlott, W. (2004) Diversity and biogeography of vascular epiphytes in Western Amazonia, Yasuní, Ecuador. Journal of Biogeography, 31, 1463-1476.

Krömer, T. \& Kessler, M. (2006) Filmy ferns (Hymenophyllaceae) as high-canopy epiphytes. Ecotropica, 12, 57-63.

Krömer, T., Kessler, M. \& Gradstein, S.R. (2007) Vertical stratification of vascular epiphytes in submontane and montane forest of the Bolivian Andes: the importance of the understory. Plant Ecology, 189, 261-278.

Krömer, T., Kessler, M., Gradstein, S.R. \& Acebey, A. (2005) Diversity patterns of vascular epiphytes along an elevational gradient in the Andes. Journal of Biogeography, 32, 1799-1809.

de Kroon, H., Huber, H., Stuefer, J.F. \& van Groenendael, J.M. (2005) A modular concept of phenotypic plasticity in plants. New Phytologist, 166, 73-82.

Küper, W., Kreft, H., Nieder, J., Barthlott, W. \& Köster, N. (2004) Large-scale diversity patterns of vascular epiphytes in Neotropical montane rain forests. Journal of Biogeography, 31, 1477-1487. 


\section{References}

Lacointe, A. (2000) Carbon allocation among tree organs: A review of basic processes and representation in functionalstructural tree models. Annals of Forest Science, 57, 521-533.

Laube, S. \& Zotz, G. (2006) Long-term changes of the vascular epiphyte assemblage on the palm Socratea exorrhiza in a lowland forest in Panama. Journal of Vegetation Science, 17, 307.

Laurance, W.F., Delamônica, P., Laurance, S.G., Vasconcelos, H.L. \& Lovejoy, T.E. (2000) Rainforest fragmentation kills big trees. Nature, 404, 836 .

Laurance, S.G.W., Laurance, W.F., Nascimento, H.E.M., Andrade, A., Fearnside, P.M., Rebello, E.R.G. \& Condit, R. (2009) Long-term variation in Amazon forest dynamics. Journal of Vegetation Science, 20, 323-333.

Leibold, M.A., Holyoak, M., Mouquet, N., Amarasekare, P., Chase, J.M., Hoopes, M.F., Holt, R.D., Shurin, J.B., Law, R., Tilman, D., Loreau, M. \& Gonzalez, A. (2004) The metacommunity concept: A framework for multiscale community ecology. Ecology Letters, 7, 601-613.

Lewis, S.L., Phillips, O.L., Baker, T.R., Lloyd, J., Malhi, Y., Almeida, S., Higuchi, N., Laurance, W.F., Neill, D.A., Silva, J.N.M., Terborgh, J., Torres Lezama, A., Vasquez Martinez, R., Brown, S., Chave, J., Kuebler, C., Nunez Vargas, P. \& Vinceti, B. (2004a) Concerted changes in tropical forest structure and dynamics: evidence from 50 South American long-term plots. Philosophical Transactions of the Royal Society B: Biological Sciences, 359, 421-436.

Lewis, S.L., Phillips, O.L., Sheil, D., Vinceti, B., Baker, T.R., Brown, S., Graham, A.W., Higuchi, N., Hilbert, D.W., Laurance, W.F., Lejoly, J., Malhi, Y., Monteagudo, A., Nunez Vargas, P., Sonke, B., Terborgh, J.W. \& Vasquez Martinez, R. (2004b) Tropical forest tree mortality, recruitment and turnover rates: calculation, interpretation and comparison when census intervals vary. Journal of Ecology, 92, 929-944.

Lieberman, D., Lieberman, M., Hartshorn, G. \& Peralta, R. (1985) Growth rates and age-size relationships of tropical wet forest trees in Costa Rica. Journal of Tropical Ecology, 1, 97-109.

Lieberman, D., Lieberman, M., Peralta, R. \& Hartshorn, G. (1996) Tropical forest structure and composition on a largescale altitudinal gradient in Costa Rica. Journal of Ecology, 84, 137-152.

Lindenmayer, A. (1968a) Mathematical models for cellular interactions in development. II. Simple and branching filaments with two-sided inputs. Journal of Theoretical Bbiology, 18, 300-315.

Lindenmayer, A. (1968b) Mathematical models for cellular interactions in development. I. Filaments with one-sided inputs. Journal of Theoretical Bbiology, 18, 280-299.

Lindenmayer, D.B., Laurance, W.F. \& Franklin, J.F. (2012) Global decline in large old trees. Science, 338, $1305-1306$.

Liu, J. (1998) FORMOSAIC: an individual-based spatially explicit model for simulating forest dynamics in landscape mosaics. Ecological Modelling, 106, 177-200.

Mäkelä, A. (1986) Implications of the pipe model theory on dry matter partitioning and height growth in trees. Journal of Theoretical Biology, 123, 103-120.

Mäkelä, A. (2002) Derivation of stem taper from the pipe theory in a carbon balance framework. Tree Physiology, 22, 891-905.

Mäkelä, A. (2012) On guiding principles for carbon allocation in eco-physiological growth models. Tree Physiology, 32, 644-647.

Malhi, Y. (2012) The productivity, metabolism and carbon cycle of tropical forest vegetation. Journal of Ecology, 100, $65-75$.

Malhi, Y., Aragão, L.E.O.C., Metcalfe, D.B., Paiva, R., Quesada, C.A., Almeida, S., Anderson, L., Brando, P., Chambers, J.Q., Da Costa, A.C.L., Hutyra, L.R., Oliveira, P., Patiño, S., Pyle, E.H., Robertson, A.L. \& 
Teixeira, L.M. (2009) Comprehensive assessment of carbon productivity, allocation and storage in three Amazonian forests. Global Change Biology, 15, 1255-1274.

Malhi, Y., Baker, T.R., Phillips, O.L., Almeida, S., Alvarez, E., Arroyo, L., Chave, J., Czimczik, C.I., Di Fiore, A., Higuchi, N., Killeen, T.J., Laurance, S.G., Laurance, W.F., Lewis, S.L., Montoya, L.M.M., Monteagudo, A., Neill, D.A., Vargas, P.N., Patino, S., Pitman, N.C.A., Quesada, C.A., Salomao, R., Silva, J.N.M., Lezama, A.T., Martinez, R.V., Terborgh, J., Vinceti, B. \& Lloyd, J. (2004) The above-ground coarse wood productivity of 104 Neotropical forest plots. Global Change Biology, 10, 563-591.

Malhi, Y., Doughty, C. \& Galbraith, D. (2011) The allocation of ecosystem net primary productivity in tropical forests. Philosophical transactions of the Royal Society B, 366, 3225-3245.

Malhi, Y., Doughty, C.E., Goldsmith, G.R., Metcalfe, D.B., Girardin, C.A.J., Marthews, T.R., del Aguila-Pasquel, J., Aragão, L.E.O.C., Araujo-Murakami, A., Brando, P., da Costa, A.C.L., Silva-Espejo, J.E., Farfán Amézquita, F., Galbraith, D.R., Quesada, C.A., Rocha, W., Salinas-Revilla, N., Silvério, D., Meir, P. \& Phillips, O.L. (2015) The linkages between photosynthesis, productivity, growth and biomass in lowland Amazonian forests. Global Change Biology, 21, 2283-2295.

Malhi, Y., Farfán Amézquita, F., Doughty, C.E., Silva-Espejo, J.E., Girardin, C.A.J., Metcalfe, D.B., Aragão, L.E.O.C., Huaraca-Quispe, L.P., Alzamora-Taype, I., Eguiluz-Mora, L., Marthews, T.R., Halladay, K., Quesada, C.A., Robertson, A.L., Fisher, J.B., Zaragoza-Castells, J., Rojas-Villagra, C.M., Pelaez-Tapia, Y., Salinas, N., Meir, P. \& Phillips, O.L. (2013) The productivity, metabolism and carbon cycle of two lowland tropical forest plots in south-western Amazonia, Peru. Plant Ecology \& Diversity, 7, 1-21.

Malhi, Y. \& Grace, J. (2000) Tropical forests and atmospheric carbon dioxide. Trends in Ecology \& Evolution, 15, 332-337.

Malhi, Y., Wood, D., Baker, T.R., Wright, J., Phillips, O.L., Cochrane, T., Meir, P., Chave, J., Almeida, S., Arroyo, L., Higuchi, N., Killeen, T.J., Laurance, S.G., Laurance, W.F., Lewis, S.L., Monteagudo, A., Neill, D.A., Vargas, P.N., Pitman, N.C.A., Quesada, C.A., Salomao, R., Silva, J.N.M., Lezama, A.T., Terborgh, J., Martinez, R.V. \& Vinceti, B. (2006) The regional variation of aboveground live biomass in old-growth Amazonian forests. Global Change Biology, 12, 1107-1138.

Mantovani, A. (1999) Leaf morpho-physiology and distribution of epiphytic aroids along a vertical gradient in a Brazilian rain forest. Selbyana, 20, 241-249.

Marin-Spiotta, E., Ostertag, R. \& Silver, W.L. (2007) Long-term patterns in tropical reforestation: Plant community composition and aboveground biomass accumulation. Ecological Applications, 17, 828-839.

Marino, G., Aqil, M. \& Shipley, B. (2010) The leaf economics spectrum and the prediction of photosynthetic lightresponse curves. Functional Ecology, 24, 263-272.

Markesteijn, L., Poorter, L. \& Bongers, F. (2007) Light-dependent leaf trait variation in 43 tropical dry forest tree species. American Journal of Botany, 94, 515-525.

Martensen, A.C., Pimentel, R.G. \& Metzger, J.P. (2008) Relative effects of fragment size and connectivity on bird community in the Atlantic rain forest: Implications for conservation. Biological Conservation, 141, 21842192.

Martin, A.R. \& Thomas, S.C. (2011) A reassessment of carbon content in tropical trees. PloS one, 6, e23533.

Martínez, I., Wiegand, T., Camarero, J.J., Batllori, E. \& Gutiérrez, E. (2011) Disentangling the formation of contrasting tree-line physiognomies combining model selection and Bayesian parameterization for simulation models. The American Naturalist, 177, E136-E152. 


\section{References}

Martínez-Ramos, M. \& Alvarez-Buylla, E.R. (1998) How old are tropical rain forest trees? Trends in Plant Science, 3, $400-404$.

Matelson, T.J., Nadkarni, N.M. \& Longino, J.T. (1993) Longevity of fallen epiphytes in a Neotropical montane forest. Ecology, 74, 265-269.

Matsushita, M., Takata, K., Hitsuma, G., Yagihashi, T., Noguchi, M., Shibata, M. \& Masaki, T. (2015) A novel growth model evaluating age-size effect on long-term trends in tree growth. Functional Ecology, 29, 1250-1259.

McCoy, M.W. \& Gillooly, J.F. (2008) Predicting natural mortality rates of plants and animals. Ecology Letters, 11, $710-716$.

McMahon, T. (1971) Size and shape in biology. Science, 179, 1201-1204.

McMurtrie, R.E. \& Dewar, R.C. (2011) Leaf-trait variation explained by the hypothesis that plants maximize their canopy carbon export over the lifespan of leaves. Tree Physiology, 31, 1007-1023.

Medina, E. \& Minchin, P. (1980) Stratification of $\delta^{13}$ C values of leaves in Amazonian rain forests. Oecologia, 378, $377-378$

Mendieta-Leiva, G. \& Zotz, G. (2015) A conceptual framework for the analysis of vascular epiphyte assemblages. Perspectives in Plant Ecology, Evolution and Systematics, 17, 510-521.

Messier, J., McGill, B.J. \& Lechowicz, M.J. (2010) How do traits vary across ecological scales? A case for trait-based ecology. Ecology Letters, 13, 838-48.

Metcalf, C.J.E., Horvitz, C.C., Tuljapurkar, S. \& Clark, D.A. (2009) A time to grow and a time to die: a new way to analyze the dynamics of size, light, age, and death of tropical trees. Ecology, 90, 2766-78.

Millington, W. \& Chaney, W. (1973) Shedding of shoots and branches. Shedding of plant parts (ed T. Kozlowski), pp. 149-204. Academic Press, New York.

Mitchard, E.T.A., Feldpausch, T.R., Brienen, R.J.W., Lopez-Gonzalez, G., Monteagudo, A., Baker, T.R., Lewis, S.L., Lloyd, J., Quesada, C.A., Gloor, M., ter Steege, H., Meir, P., Alvarez, E., Araujo-Murakami, A., Aragão, L.E.O.C., Arroyo, L., Aymard, G., Banki, O., Bonal, D., Brown, S., Brown, F.I., Cerón, C.E., Chama Moscoso, V., Chave, J., Comiskey, J.A., Cornejo, F., Corrales Medina, M., Da Costa, L., Costa, F.R.C., Di Fiore, A., Domingues, T.F., Erwin, T.L., Frederickson, T., Higuchi, N., Honorio Coronado, E.N., Killeen, T.J., Laurance, W.F., Levis, C., Magnusson, W.E., Marimon, B.S., Marimon Junior, B.H., Mendoza Polo, I., Mishra, P., Nascimento, M.T., Neill, D., Núñez Vargas, M.P., Palacios, W.A., Parada, A., Pardo Molina, G., Peña-Claros, M., Pitman, N., Peres, C.A., Poorter, L., Prieto, A., Ramirez-Angulo, H., Restrepo Correa, Z., Roopsind, A., Roucoux, K.H., Rudas, A., Salomão, R.P., Schietti, J., Silveira, M., de Souza, P.F., Steininger, M.K., Stropp, J., Terborgh, J., Thomas, R., Toledo, M., Torres-Lezama, A., van Andel, T.R., van der Heijden, G.M.F., Vieira, I.C.G., Vieira, S., Vilanova-Torre, E., Vos, V.A., Wang, O., Zartman, C.E., Malhi, Y. \& Phillips, O.L. (2014) Markedly divergent estimates of Amazon forest carbon density from ground plots and satellites. Global Ecology and Biogeography, 23, 935-946.

Mitchell Aide, T., Zimmerman, J.K., Pascarella, J.B., Rivera, L. \& Marcano-Vega, H. (2000) Forest regeneration in a chronosequence of tropical abandoned pastures: Implications for restoration ecology. Restoration Ecology, 8, 328-338.

Moffett, M.W. (2000) What's up? A critical look at the basic terms of canopy biology. Biotropica, 32, 569-596.

Mondragón, D. \& Ticktin, T. (2011) Demographic effects of harvesting epiphytic bromeliads and an alternative approach to collection. Conservation Biology, 25, 797-807. 
Mondragón, D., Valverde, T. \& Hernández-Apolinar, M. (2015) Population ecology of epiphytic angiosperms: A review. Tropical Ecology, 56, 1-39.

Muller-Landau, H.C., Condit, R.S., Chave, J., Thomas, S.C., Bohlman, S.A., Bunyavejchewin, S., Davies, S., Foster, R., Gunatilleke, S., Gunatilleke, N., Harms, K.E., Hart, T., Hubbell, S.P., Itoh, A., Kassim, A.R., LaFrankie, J.V., Lee, H.S., Losos, E., Makana, J.R., Ohkubo, T., Sukumar, R., Sun, I.F., Nur Supardi, M.N., Tan, S., Thompson, J., Valencia, R., Muñoz, G.V., Wills, C., Yamakura, T., Chuyong, G., Dattaraja, H.S., Esufali, S., Hall, P., Hernandez, C., Kenfack, D., Kiratiprayoon, S., Suresh, H.S., Thomas, D., Vallejo, M.I. \& Ashton, P. (2006a) Testing metabolic ecology theory for allometric scaling of tree size, growth and mortality in tropical forests. Ecology Letters, 9, 575-588.

Muller-Landau, H.C., Condit, R.S., Harms, K.E., Marks, C.O., Thomas, S.C., Bunyavejchewin, S., Chuyong, G., Co, L., Davies, S., Foster, R., Gunatilleke, S., Gunatilleke, N., Hart, T., Hubbell, S.P., Itoh, A., Kassim, A.R., Kenfack, D., LaFrankie, J. V, Lagunzad, D., Lee, H.S., Losos, E., Makana, J.-R., Ohkubo, T., Samper, C., Sukumar, R., Sun, I.-F., Nur Supardi, M.N., Tan, S., Thomas, D., Thompson, J., Valencia, R., Vallejo, M.I., Muñoz, G.V., Yamakura, T., Zimmerman, J.K., Dattaraja, H.S., Esufali, S., Hall, P., He, F., Hernandez, C., Kiratiprayoon, S., Suresh, H.S., Wills, C. \& Ashton, P. (2006b) Comparing tropical forest tree size distributions with the predictions of metabolic ecology and equilibrium models. Ecology Letters, 9, 589-602.

Myers, N., Mittermeier, R.A., Mittermeier, C.G., da Fonseca, G.A.B. \& Kent, J. (2000) Biodiversity hotspots for conservation priorities. Nature, 403, 853-858.

Myneni, R.B., Yang, W., Nemani, R.R., Huete, A.R., Dickinson, R.E., Knyazikhin, Y., Didan, K., Fu, R., Negrón Juárez, R.I., Saatchi, S.S., Hashimoto, H., Ichii, K., Shabanov, N. V, Tan, B., Ratana, P., Privette, J.L., Morisette, J.T., Vermote, E.F., Roy, D.P., Wolfe, R.E., Friedl, M.A., Running, S.W., Votava, P., El-Saleous, N., Devadiga, S., Su, Y. \& Salomonson, V.V. (2007) Large seasonal swings in leaf area of Amazon rainforests. Proceedings of the National Academy of Sciences of the United States of America, 104, 48204823.

Nadkarni, N.M. \& Matelson, T.J. (1992) Biomass and nutrient dynamics of epiphytic litterfall in a Neotropical montane forest, Costa Rica. Biotropica, 24, 24-30.

Nicolini, E., Caraglio, Y., Pelissier, R., Leroy, C. \& Roggy, J.-C. (2003) Epicormic branches: a growth indicator for the tropical forest tree, Dicorynia guianensis Amshoff (Caesalpiniaceae). Annals of Botany, 92, 97-105.

Nieder, J., Engwald, S., Klawun, M. \& Barthlott, W. (2000) Spatial distribution of vascular epiphytes (including hemiepiphytes) in a lowland Amazonian rain forest (Surumoni crane plot) of Southern Venezuela. Biotropica, 32, 385-396.

Niinemets, Ü., Keenan, T.F. \& Halllik, L. (2015) A worldwide analysis of within-canopy variations in leaf structural, chemical and physiological traits across plant functional types. New Phytologist, 205, 973-993.

Niklas, K.J. (1995) Size-dependent allometry of tree height, diameter and truck-taper. Annals of Botany, 75, $217-227$.

O’Hara, R.B. \& Kotze, D.J. (2010) Do not log-transform count data. Methods in Ecology and Evolution, 1, 118-122.

Oliveira-Filho, A.T., de Mello, J.M. \& Scolforo, J.R.S. (1997) Effects of past disturbance and edges on tree community structure and dynamics within a fragment of tropical semideciduous forest in south-eastern Brazil over a fiveyear period (1987-1992). Plant Ecology, 131, 45-66.

Oliveira-Filho, A.T., Vilela, E.A, Carvalho, D.A. \& Gavilanes, M.L. (1994) Effects of soils and topography on the distribution of tree species in a tropical riverine forest in south-eastern Brazil. Journal of Tropical Ecology, 10, 483-503. 


\section{References}

Oliveira, M.A., Grillo, A.S. \& Tabarelli, M. (2004) Forest edge in the Brazilian Atlantic forest: drastic changes in tree species assemblages. Oryx, 38, 389-394.

Osada, N. (2011) Height-dependent changes in shoot structure and tree allometry in relation to maximum height in four deciduous tree species. Functional Ecology, 25, 777-786.

Osunkoya, O.O., Omar-Ali, K., Amit, N., Dayan, J., Daud, D.S. \& Sheng, T.K. (2007) Comparative height crown allometry and mechanical design in 22 tree species of Kuala Belalong rainforest, Brunei, Borneo. American Journal of Botany, 94, 1951-1962.

Padmawathe, R. (2004) Effects of selective logging on vascular epiphyte diversity in a moist lowland forest of Eastern Himalaya, India. Biological Conservation, 119, 81-92.

Pan, Y., Birdsey, R.A., Phillips, O.L. \& Jackson, R.B. (2013) The Structure, distribution, and biomass of the world's Forests. Annual Review of Ecology, Evolution, and Systematics, 44, 593-622.

Pardini, R., De Souza, S.M., Braga-Neto, R. \& Metzger, J.P. (2005) The role of forest structure, fragment size and corridors in maintaining small mammal abundance and diversity in an Atlantic forest landscape. Biological Conservation, 124, 253-266.

Parker, G. (1995) Structure and microclimate of forest canopies. Forest Canopies (eds M. Lowman \& N. Nadkarni), pp. 73-106. Academic Press, San Diego, USA.

Patiño, S., Fyllas, N.M., Baker, T.R., Paiva, R., Quesada, C.A., Santos, A.J.B., Schwarz, M., Ter Steege, H., Phillips, O.L. \& Lloyd, J. (2012) Coordination of physiological and structural traits in Amazon forest trees. Biogeosciences, 9, 775-801.

Patiño, S., Lloyd, J., Paiva, R., Baker, T.R., Quesada, C.A., Mercado, L.M., Schmerler, J., Schwarz, M., Santos, A.J.B., Aguilar, A., Czimczik, C.I., Gallo, J., Horna, V., Hoyos, E.J., Jimenez, E.M., Palomino, W., Peacock, J., Peña-Cruz, A., Sarmiento, C., Sota, A., Turriago, J.D., Villanueva, B., Vitzthum, P., Alvarez, E., Arroyo, L., Baraloto, C., Bonal, D., Chave, J., Costa, A.C.L., Herrera, R., Higuchi, N., Killeen, T., Leal, E., Luizão, F., Meir, P., Monteagudo, A., Neil, D., Núñez-Vargas, P., Peñuela, M.C., Pitman, N., Priante Filho, N., Prieto, A., Panfil, S.N., Rudas, A., Salomão, R., Silva, N., Silveira, M., Soares de Almeida, S., Torres-Lezama, A., Vásquez-Martínez, R., Vieira, I., Malhi, Y. \& Phillips, O.L. (2009) Branch xylem density variations across the Amazon Basin. Biogeosciences, 6, 545-568.

Penning De Vries, F.W.T. (1975) The cost of maintenance processes in plant cells. Annals of Botany, 39, 77-92.

Perttunen, J., Sievänen, R. \& Nikinmaa, E. (1998) LIGNUM : a model combining the structure and the functioning of trees. Ecological Modelling, 108, 189-198.

Perttunen, J., Sievänen, R., Nikinmaa, E., Salminen, H., Saarenmaa, H. \& Väkevä, J. (1996) LIGNUM: a tree model based on simple structural units. Annals of Botany, 77, 87-98.

Petter, G., Wagner, K., Wanek, W., Sánchez Delgado, E.J., Zotz, G., Cabral, J.S. \& Kreft, H. (2016) Functional leaf traits of vascular epiphytes: vertical trends within the forest, intra- and interspecific trait variability, and taxonomic signals. Functional Ecology, 30, 188-198.

Philipson, C.D., Dent, D.H., O’Brien, M.J., Chamagne, J., Dzulkifli, D., Nilus, R., Philips, S., Reynolds, G., Saner, P. \& Hector, A. (2014) A trait-based trade-off between growth and mortality: evidence from 15 tropical tree species using size-specific relative growth rates. Ecology and Evolution, 4, 3675-3688.

Phillips, O.L. (1996) Long-term environmental change in tropical forests: increasing tree turnover. Environmental Conservation, 23, 235. 
Phillips, P.D, de Azevedo, C.P., Degen, B., Thompson, I.S., Silva, J.N.M. \& van Gardingen, P.R. (2004a) An individual-based spatially explicit simulation model for strategic forest management planning in the eastern Amazon. Ecological Modelling, 173, 335-354.

Phillips, O.L., Baker, T.R., Arroyo, L., Higuchi, N., Killeen, T.J., Laurance, W.F., Lewis, S.L., Lloyd, J., Malhi, Y., Monteagudo, A., Neill, D.A., Nunez Vargas, P., Silva, J.N.M., Terborgh, J., Vasquez Martinez, R., Alexiades, M., Almeida, S., Brown, S., Chave, J., Comiskey, J.A., Czimczik, C.I., Di Fiore, A., Erwin, T., Kuebler, C., Laurance, S.G., Nascimento, H.E.M., Olivier, J., Palacios, W., Patino, S., Pitman, N.C.A., Quesada, C.A., Saldias, M., Torres Lezama, A. \& Vinceti, B. (2004b) Pattern and process in Amazon tree turnover, 19762001. Philosophical Transactions of the Royal Society B: Biological Sciences, 359, 381-407.

Phillips, O.L., Hall, P., Gentry, A.H., Sawyer, S.A. \& Vásquez, R. (1994) Dynamics and species richness of tropical rain forests. Proceedings of the National Academy of Sciences of the United States of America, 91, 28052809.

Phillips, O.L., Higuchi, N., Vieira, S., Baker, T.R., Chao, K.J. \& Lewis, S.L. (2009) Changes in Amazonian forest biomass, dynamics, and composition, 1980-2002. Geophysical Monograph Series, 186, 373-387.

Pons, T.L. \& Poorter, H. (2014) The effect of irradiance on the carbon balance and tissue characteristics of five herbaceous species differing in shade-tolerance. Frontiers in Plant Science, 5, 1-14.

Poorter, L. (1999) Growth responses of 15 rain-forest tree species to a light gradient: the relative importance of morphological and physiological traits. Functional Ecology, 13, 396-410.

Poorter, L. \& Bongers, F. (2006) Leaf traits are good predictors of plant performance across 53 rain forest species. Ecology, 87, 1733-43.

Poorter, L., Bongers, L. \& Bongers, F. (2006) Architecture of 54 moist-forest tree species: traits, trade-offs, and functional groups. Ecology, 87, 1289-1301.

Poorter, L., Bongers, F., Sterck, F.J. \& Wöll, H. (2011) Architecture of 53 rain forest tree species differing in adult stature and shade tolerance. Ecology, 84, 602-608.

Poorter, H. \& De Jong, R. (1999) A comparison of specific leaf area, chemical composition and leaf construction costs of field plants from 15 habitats differing in productivity. New Phytologist, 143, 163-176.

Poorter, L., Lianes, E., Moreno-de las Heras, M. \& Zavala, M.A. (2012) Architecture of Iberian canopy tree species in relation to wood density, shade tolerance and climate. Plant Ecology, 213, 707-722.

Poorter, H., Niinemets, Ü., Poorter, L., Wright, I.J. \& Villar, R. (2009) Causes and consequences of variation in leaf mass per area (LMA): a meta-analysis. New Phytologist, 182, 565-588.

Prance, G.T. (1982) A review of the phytogeographic evidences for pleistocene climate changes in the Neotropics. Annals of the Missouri Botanical Garden, 69, 594-624.

Purves, D. \& Pacala, S. (2008) Predictive models of forest dynamics. Science, 320, 1452-1453.

Quay, P., King, S. \& Wilbur, D. (1989) ${ }^{13} \mathrm{C} /{ }^{12} \mathrm{C}$ of atmospheric $\mathrm{CO}_{2}$ in the Amazon basin: Forest and river sources. Journal of Geophysical Research, 94, 18327-18336.

Quesada, C.A., Phillips, O.L., Schwarz, M., Czimczik, C.I., Baker, T.R., Patiño, S., Fyllas, N.M., Hodnett, M.G., Herrera, R., Almeida, S., Alvarez Dávila, E., Arneth, A., Arroyo, L., Chao, K.J., Dezzeo, N., Erwin, T., di Fiore, A., Higuchi, N., Honorio Coronado, E., Jimenez, E.M., Killeen, T., Lezama, A.T., Lloyd, G., LópezGonzález, G., Luizão, F.J., Malhi, Y., Monteagudo, A., Neill, D.A., Núñez Vargas, P., Paiva, R., Peacock, J., Peñuela, M.C., Peña Cruz, A., Pitman, N., Priante Filho, N., Prieto, A., Ramírez, H., Rudas, A., Salomão, R., Santos, A.J.B., Schmerler, J., Silva, N., Silveira, M., Vásquez, R., Vieira, I., Terborgh, J. \& Lloyd, J. (2012) 


\section{References}

Basin-wide variations in Amazon forest structure and function are mediated by both soils and climate. Biogeosciences, 9, 2203-2246.

Raich, J.W., Russell, A.E., Kitayama, K., Parton, W.J. \& Vitousek, P.M. (2006) Temperature influences carbon accumulation in moist tropical forests. Ecology, 87, 76-87.

Rijkers, T., Pons, T.L. \& Bongers, F. (2000) The effect of tree height and light availability on photosynthetic leaf traits of four Neotropical species differing in shade tolerance. Functional Ecology, 14, 77-86.

Rozendaal, D.M.A., Hurtado, V.H. \& Poorter, L. (2006) Plasticity in leaf traits of 38 tropical tree species in response to light; relationships with light demand and adult stature. Functional Ecology, 20, 207-216.

Rüger, N., Berger, U., Hubbell, S.P., Vieilledent, G. \& Condit, R. (2011) Growth strategies of tropical tree species: disentangling light and size effects. PloS one, 6, e25330.

Rüger, N., Wirth, C., Wright, S.J. \& Condit, R. (2012) Functional traits explain light and size response of growth rates in tropical tree species. Ecology, 93, 2626-2636.

Rust, S. \& Roloff, A. (2004) Acclimation of crown structure to drought in Quercus robur L. - Intra- and inter-annual variation of abscission and traits of shed twigs. Basic and Applied Ecology, 5, 283-291.

Ryan, M.G., Gower, S.T., Hubbard, R.M., Waring, R.H., Gholz, H.L., Cropper, W.P. \& Running, S.W. (1995) Woody tissue maintenance respiration of four conifers in contrasting climates. Oecologia, 101, 133-140.

Ryan, M.G., Hubbard, R.M., Clark, D.A. \& Sanford, R.L. (1994) Woody-tissue respiration for Simarouba amara and Minquartia guianensis, two tropical wet forest trees with different growth habits. Oecologia, 100, 213-220.

Saldarriaga, J.G., West, D.C., Tharp, M.L. \& Uhl, C. (1988) Long-term chronosequence of forest succession in the upper Rio Negro of Colombia and Venezuela. Journal of Ecology, 76, 938-958.

Sarmento Cabral, J., Petter, G., Mendieta-Leiva, G., Wagner, K., Zotz, G. \& Kreft, H. (2015) Branchfall as a demographic filter for epiphyte communities: lessons from forest floor-based sampling. PloS one, 10, e0128019.

Sawada, Y., Suwa, R., Jindo, K., Endo, T., Oki, K., Sawada, H., Arai, E., Shimabukuro, Y.E., Celes, C.H.S., Campos, M.A.A., Higuchi, F.G., Lima, A.J.N., Higuchi, N., Kajimoto, T. \& Ishizuka, M. (2015) A new 500-m resolution map of canopy height for Amazon forest using spaceborne LiDAR and cloud-free MODIS imagery. International Journal of Applied Earth Observation and Geoinformation, 43, 92-101.

Schimper, A.F.W. (1888) Die epiphytische Vegetation Amerikas. Gustav Fischer, Jena.

Schmit-Neuerburg, V. (2002) Dynamics of vascular epiphyte vegetation in the Venezuelan lowland rain forest of the Surumoni Crane Project. PhD Thesis, University of Bonn.

Seyoum, F., Fetene, M., Strobl, S. \& Beck, E. (2014) Daily and seasonal courses of gas exchange and niche partitioning among coexisting tree species in a tropical montane forest. Flora, 209, 191-200.

Shaw, J.D. \& Bergstrom, D.M. (1997) A rapid assessment technique of vascular epiphyte diversity at forest and regional levels. Selbyana, 18, 195-199.

Shinozaki, K., Yoda, K., Hozumi, K. \& Kira, T. (1964) A quantitative analysis of plant form-the pipe model theory I. Basic analyses. Japanese Journal of Ecology, 14, 97-105.

Shipley, B., Lechowicz, M.J., Wright, I. \& Reich, P.B. (2006) Fundamental trade-offs generating the worldwide leaf economics spectrum. Ecology, 87, 535-541. 
Sides, C.B., Enquist, B.J., Ebersole, J.J., Smith, M.N., Henderson, A.N. \& Sloat, L.L. (2014) Revisiting Darwin's hypothesis: does greater intraspecific variability increase species' ecological breadth? American Journal of Botany, 101, 56-62.

Sievänen, R., Godin, C., DeJong, T.M. \& Nikinmaa, E. (2014) Functional-structural plant models: a growing paradigm for plant studies. Annals of Botany, 114, 599-603.

da Silva, J.M.C. \& Tabarelli, M. (2000) Tree species impoverishment and the future flora of the Atlantic forest of northeast Brazil. Nature, 404, 72-74.

Silvertown, J., Poulton, P., Johnston, E., Edwards, G., Heard, M. \& Biss, P.M. (2006) The Park Grass Experiment 1856-2006: Its contribution to ecology. Journal of Ecology, 94, 801-814.

Simard, M., Pinto, N., Fisher, J.B. \& Baccini, A. (2011) Mapping forest canopy height globally with spaceborne lidar. Journal of Geophysical Research, 116, 1-12.

Sims, D.A., Gebauer, R.L.E. \& Pearcy, R.W. (1994) Scaling sun and shade photosynthetic acclimation of Alocasia macrorrhiza to whole plant performance - II. Simulation of carbon balance and growth at different photon flux densities. Plant, Cell \& Environment, 17, 889-900.

Siqueira Filho, J.A. \& Félix, L.P. (2005) Bromélias e orquídeas. Diversidade Biológica do Centro de endemismo Pernambuco. Brasília: Ministério do Meio Ambiente (eds K.C. Porto, J.S. de Almelda-Cortez \& M. Tabarelli), pp. 125-133. Brasília.

Siqueira Filho, J.A., Santos, A.M., Leme, E.M. \& Cabral, J.S. (2006) Atlantic forest fragments and bromeliads in Pernambuco and Alagoas: distribution, composition, richness and conservation. (eds J.A. Siqueira Filho \& A.M. Santos), pp. 101-131. Andrea Jakobsson Estúdio, Rio de Janeiro.

Slik, J.W.F., Arroyo-Rodríguez, V., Aiba, S., Alvarez-Loayza, P., Alves, L.F., Ashton, P., Balvanera, P., Bastian, M.L., Bellingham, P.J., van den Berg, E., Bernacci, L., da Conceição Bispo, P., Blanc, L., Böhning-Gaese, K., Boeckx, P., Bongers, F., Boyle, B., Bradford, M., Brearley, F.Q., Breuer-Ndoundou Hockemba, M., Bunyavejchewin, S., Calderado Leal Matos, D., Castillo-Santiago, M., Catharino, E.L.M., Chai, S.-L., Chen, Y., Colwell, R.K., Robin, C.L., Clark, C., Clark, D.B., Clark, D.A., Culmsee, H., Damas, K., Dattaraja, H.S., Dauby, G., Davidar, P., DeWalt, S.J., Doucet, J.-L., Duque, A., Durigan, G., Eichhorn, K.A.O., Eisenlohr, P. V, Eler, E., Ewango, C., Farwig, N., Feeley, K.J., Ferreira, L., Field, R., de Oliveira Filho, A.T., Fletcher, C., Forshed, O., Franco, G., Fredriksson, G., Gillespie, T., Gillet, J.-F., Amarnath, G., Griffith, D.M., Grogan, J., Gunatilleke, N., Harris, D., Harrison, R., Hector, A., Homeier, J., Imai, N., Itoh, A., Jansen, P.A., Joly, C.A., de Jong, B.H.J., Kartawinata, K., Kearsley, E., Kelly, D.L., Kenfack, D., Kessler, M., Kitayama, K., Kooyman, R., Larney, E., Laumonier, Y., Laurance, S., Laurance, W.F., Lawes, M.J., Amaral, I.L. do, Letcher, S.G., Lindsell, J., Lu, X., Mansor, A., Marjokorpi, A., Martin, E.H., Meilby, H., Melo, F.P.L., Metcalfe, D.J., Medjibe, V.P., Metzger, J.P., Millet, J., Mohandass, D., Montero, J.C., de Morisson Valeriano, M., Mugerwa, B., Nagamasu, H., Nilus, R., Ochoa-Gaona, S., Onrizal, Page, N., Parolin, P., Parren, M., Parthasarathy, N., Paudel, E., Permana, A., Piedade, M.T.F., Pitman, N.C.A., Poorter, L., Poulsen, A.D., Poulsen, J., Powers, J., Prasad, R.C., Puyravaud, J.-P., Razafimahaimodison, J.-C., Reitsma, J., Dos Santos, J.R., Roberto Spironello, W., Romero-Saltos, H., Rovero, F., Rozak, A.H., Ruokolainen, K., Rutishauser, E., Saiter, F., Saner, P., Santos, B.A., Santos, F., Sarker, S.K., Satdichanh, M., Schmitt, C.B., Schöngart, J., Schulze, M., Suganuma, M.S., Sheil, D., da Silva Pinheiro, E., Sist, P., Stevart, T., Sukumar, R., Sun, I.-F., Sunderand, T., Suresh, H.S., Suzuki, E., Tabarelli, M., Tang, J., Targhetta, N., Theilade, I., Thomas, D.W., Tchouto, P., Hurtado, J., Valencia, R., van Valkenburg, J.L.C.H., Van Do, T., Vásquez, R., Verbeeck, H., Adekunle, V., Vieira, S.A., Webb, C.O., Whitfeld, T., Wich, S.A., Williams, J., Wittmann, F., Wöll, H., Yang, X., Adou Yao, C.Y., Yap, S.L., Yoneda, T., Zahawi, R.A., Zakaria, R., Zang, R., de Assis, R.L., Garcia 


\section{References}

Luize, B. \& Venticinque, E.M. (2015) An estimate of the number of tropical tree species. Proceedings of the National Academy of Sciences of the United States of America, 112, 7472-7477.

Slik, J.W.F., Paoli, G., McGuire, K., Amaral, I., Barroso, J., Bastian, M., Blanc, L., Bongers, F., Boundja, P., Clark, C., Collins, M., Dauby, G., Ding, Y., Doucet, J.-L., Eler, E., Ferreira, L., Forshed, O., Fredriksson, G., Gillet, J.-F., Harris, D., Leal, M., Laumonier, Y., Malhi, Y., Mansor, A., Martin, E., Miyamoto, K., AraujoMurakami, A., Nagamasu, H., Nilus, R., Nurtjahya, E., Oliveira, Á., Onrizal, O., Parada-Gutierrez, A., Permana, A., Poorter, L., Poulsen, J., Ramirez-Angulo, H., Reitsma, J., Rovero, F., Rozak, A., Sheil, D., Silva-Espejo, J., Silveira, M., Spironelo, W., ter Steege, H., Stevart, T., Navarro-Aguilar, G.E., Sunderland, T., Suzuki, E., Tang, J., Theilade, I., van der Heijden, G., van Valkenburg, J., Van Do, T., Vilanova, E., Vos, V., Wich, S., Wöll, H., Yoneda, T., Zang, R., Zhang, M.-G. \& Zweifel, N. (2013) Large trees drive forest aboveground biomass variation in moist lowland forests across the tropics. Global Ecology and Biogeography, 22, 1261-1271.

Sprugel, D.G., Hinckley, T.M. \& Schaap, W. (1991) The theory and practice of branch autonomy. Annual Review of Ecology and Systematics, 22, 309-334.

Stanton, D.E., Huallpa Chávez, J., Villegas, L., Villasante, F., Armesto, J., Hedin, L.O. \& Horn, H. (2014) Epiphytes improve host plant water use by microenvironment modification. Functional Ecology, 28, 1274-1283.

Stark, S.C., Leitold, V., Wu, J.L., Hunter, M.O., de Castilho, C. V., Costa, F.R.C., Mcmahon, S.M., Parker, G.G., Shimabukuro, M.T., Lefsky, M.A., Keller, M., Alves, L.F., Schietti, J., Shimabukuro, Y.E., Brandão, D.O., Woodcock, T.K., Higuchi, N., de Camargo, P.B., de Oliveira, R.C. \& Saleska, S.R. (2012) Amazon forest carbon dynamics predicted by profiles of canopy leaf area and light environment. Ecology Letters, 15, 14061414 .

ter Steege, H. \& Cornelissen, J.H.C. (1989) Distribution and ecology of vascular epiphytes in lowland rain forest of Guyana. Biotropica, 21, 331-339.

ter Steege, H., Pitman, N.C.A, Sabatier, D., Baraloto, C., Salomão, R.P., Guevara, J.E., Phillips, O.L., Castilho, C.V, Magnusson, W.E., Molino, J.-F., Monteagudo, A., Núñez Vargas, P., Montero, J.C., Feldpausch, T.R., Coronado, E.N.H., Killeen, T.J., Mostacedo, B., Vasquez, R., Assis, R.L., Terborgh, J., Wittmann, F., Andrade, A., Laurance, W.F., Laurance, S.G.W., Marimon, B.S., Marimon, B.-H., Guimarães Vieira, I.C., Amaral, I.L., Brienen, R., Castellanos, H., Cárdenas López, D., Duivenvoorden, J.F., Mogollón, H.F., Matos, F.D.D.A., Dávila, N., García-Villacorta, R., Stevenson Diaz, P.R., Costa, F., Emilio, T., Levis, C., Schietti, J., Souza, P., Alonso, A., Dallmeier, F., Montoya, A.J.D., Fernandez Piedade, M.T., Araujo-Murakami, A., Arroyo, L., Gribel, R., Fine, P.V.A., Peres, C.A, Toledo, M., Aymard, C.G.A, Baker, T.R., Cerón, C., Engel, J., Henkel, T.W., Maas, P., Petronelli, P., Stropp, J., Zartman, C.E., Daly, D., Neill, D., Silveira, M., Paredes, M.R., Chave, J., Lima Filho, D.D.A., Jørgensen, P.M., Fuentes, A., Schöngart, J., Cornejo Valverde, F., Di Fiore, A., Jimenez, E.M., Peñuela Mora, M.C., Phillips, J.F., Rivas, G., van Andel, T.R., von Hildebrand, P., Hoffman, B., Zent, E.L., Malhi, Y., Prieto, A., Rudas, A., Ruschell, A.R., Silva, N., Vos, V., Zent, S., Oliveira, A.A., Schutz, A.C., Gonzales, T., Trindade Nascimento, M., Ramirez-Angulo, H., Sierra, R., Tirado, M., Umaña Medina, M.N., van der Heijden, G., Vela, C.I.A., Vilanova Torre, E., Vriesendorp, C., Wang, O., Young, K.R., Baider, C., Balslev, H., Ferreira, C., Mesones, I., Torres-Lezama, A., Urrego Giraldo, L.E., Zagt, R., Alexiades, M.N., Hernandez, L., Huamantupa-Chuquimaco, I., Milliken, W., Palacios Cuenca, W., Pauletto, D., Valderrama Sandoval, E., Valenzuela Gamarra, L., Dexter, K.G., Feeley, K., Lopez-Gonzalez, G. \& Silman, M.R. (2013) Hyperdominance in the Amazonian tree flora. Science, 342, 12430921-12430929.

Stephenson, N.L. \& Van Mantgem, P.J. (2005) Forest turnover rates follow global and regional patterns of productivity. Ecology Letters, 8, 524-531. 
Sterck, F.J. \& Bongers, F. (1998) Ontogenetic changes in size, allometry, and mechanical design of tropical rain forest trees. American Journal of Botany, 85, 266-272.

Sterck, F.J. \& Bongers, F. (2001) Crown development in tropical rain forest trees: patterns with tree height and light availability. Journal of Ecology, 89, 1-13.

Sterck, F., Markesteijn, L., Schieving, F. \& Poorter, L. (2011) Functional traits determine trade-offs and niches in a tropical forest community. Proceedings of the National Academy of Sciences of the United States of America, 108, 20627-20632.

Sterck, F.J., Poorter, L. \& Schieving, F. (2006) Leaf traits determine the growth-survival trade-off across rain forest tree species. The American Naturalist, 167, 758-765.

Sterck, F.J. \& Schieving, F. (2007) 3-D growth patterns of trees: effects of carbon economy, meristem activity, and selection. Ecological Monographs, 77, 405-420.

Sterck, F.J., Schieving, F., Lemmens, A. \& Pons, T.L. (2005) Performance of trees in forest canopies: explorations with a bottom-up functional-structural plant growth model. New Phytologist, 166, 827-843.

Stoll, P. \& Schmid, B. (1998) Plant foraging and dynamic competition between branches of Pinus sylvestris in contrasting light environments. Journal of Ecology, 86, 934-945.

Stuntz, S., Ziegler, C., Simon, U. \& Zotz, G. (2002) Diversity and structure of the arthropod fauna within three canopy epiphyte species in central Panama. Journal of Tropical Ecology, 18, 161-176.

Stuntz, S. \& Zotz, G. (2001) Photosynthesis in vascular epiphytes: a survey of 27 species of diverse taxonomic origin. Flora, 196, 132-141.

Swaine, M.D. \& Whitmore, T.C. (1988) On the definition of ecological species groups in tropical rain forests. Vegetatio, $\mathbf{7 5}, 81-86$.

Tabarelli, M., Mantovani, W. \& Peres, C.A. (1999) Effects of habitat fragmentation on plant guild structure in the montane Atlantic forest of southeastern Brazil. Biological Conservation, 91, 119-127.

Taubert, F., Jahn, M.W., Dobner, H.-J., Wiegand, T. \& Huth, A. (2015) The structure of tropical forests and sphere packings. Proceedings of the National Academy of Sciences of the United States of America, 112, 1512515129.

Taylor, A. \& Burns, K. (2015) Epiphyte community development throughout tree ontogeny: an island ontogeny framework. Journal of Vegetation Science, 26, 902-910.

Tietjen, B. \& Huth, A. (2006) Modelling dynamics of managed tropical rainforests - An aggregated approach. Ecological Modelling, 199, 421-432.

Toledo-Aceves, T., García-Franco, J.G. \& López-Barrera, F. (2014) Bromeliad rain: An opportunity for cloud forest management. Forest Ecology and Management, 329, 129-136.

Trapnell, D.W., Hamrick, J.L., Ishibashi, C.D. \& Kartzinel, T.R. (2013) Genetic inference of epiphytic orchid colonization; it may only take one. Molecular Ecology, 22, 3680-3692.

Trapnell, D.W., Hamrick, J.L. \& Nason, J.D. (2004) Three-dimensional fine-scale genetic structure of the Neotropical epiphytic orchid, Laelia rubescens. Molecular Ecology, 13, 1111-1118.

Turner, I.M. (1996) Species loss in fragments of tropical rain forest: a review of the evidence. Journal of Applied Ecology, 33, 200-209.

van der Meer, P. \& Bongers, F. (1996) Patterns of tree-fall and branch-fall in a tropical rain forest in French Guiana. Journal of Ecology, 84, 19-29. 


\section{References}

van Valen, L. (1965) Morphological variation and width of ecological niche. American Naturalist, 99, 377-390.

Valladares, F., Allen, M.T. \& Pearcy, R.W. (1997) Photosynthetic responses to dynamic light under field conditions in six tropical rainforest shrubs occuring along a light gradient. Oecologia, 111, 505-514.

Violle, C., Navas, M.-L., Vile, D., Kazakou, E., Fortunel, C., Hummel, I. \& Garnier, E. (2007) Let the concept of trait be functional! Oikos, 116, 882-892.

Visser, M.D., Bruijning, M., Wright, S.J., Muller-Landau, H.C., Jongejans, E., Comita, L.S. \& de Kroon, H. (2016) Functional traits as predictors of vital rates across the life-cycle of tropical trees. Functional Ecology, 30,168180.

Vose, J.M. \& Ryan, M.G. (2002) Seasonal respiration of foliage, fine roots, and woody tissues in relation to growth, tissue N, and photosynthesis. Global Change Biology, 8, 182-193.

Wagner, K., Bogusch, W. \& Zotz, G. (2013) The role of the regeneration niche for the vertical stratification of vascular epiphytes. Journal of Tropical Ecology, 29, 277-290.

Wagner, K., Mendieta-Leiva, G. \& Zotz, G. (2015) Host specificity in vascular epiphytes: a review of methodology, empirical evidence and potential mechanisms. AoB PLANTS, 7, plu092.

Wanek, W., Arndt, S.K., Huber, W. \& Popp, M. (2002) Nitrogen nutrition during ontogeny of hemiepiphytic Clusia species. Functional Plant Biology, 29, 733-740.

Wania, R., Hietz, P. \& Wanek, W. (2002) Natural ${ }^{15} \mathrm{~N}$ abundance of epiphytes depends on the position within the forest canopy: source signals and isotope fractionation. Plant, Cell and Environment, 25, 581-589.

Werner, F.A. \& Gradstein, S.R. (2009) Diversity of dry forest epiphytes along a gradient of human disturbance in the tropical Andes. Journal of Vegetation Science, 20, 59-68.

West, G.B., Brown, J.H. \& Enquist, B.J. (1999) A general model for the structure and allometry of plant vascular systems. Nature, 400, 664-667.

West, G.B., Enquist, B.J. \& Brown, J.H. (2009) A general quantitative theory of forest structure and dynamics. Proceedings of the National Academy of Sciences of the United States of America, 106, 7040-7045.

Whitmore, T.C. (1990) An Introduction to Tropical Train Forests. Clarendon Press, Oxford, UK.

Wiegand, T., Jeltsch, F., Hanski, I. \& Grimm, V. (2003) Using pattern-oriented modeling for revealing hidden information: a key for reconciling ecological theory and application. Oikos, 100, 209-222.

Wilson, B.F. (2000) Apical control of branch growth and angle in woody plants. American Journal of Botany, 87, 601607.

Wilson, P.J., Thompson, K. \& Hodgson, J.G. (1999) Specific leaf area and leaf dry matter content as alternative predictors of plant strategies. New Phytologist, 143, 155-162.

Winkler, M., Hülber, K. \& Hietz, P. (2005) Effect of canopy position on germination and seedling survival of epiphytic bromeliads in a Mexican humid montane forest. Annals of Botany, 95, 1039-1047.

Wolf, J.H.D., Gradstein, S.R. \& Nadkarni, N.M. (2009) A protocol for sampling vascular epiphyte richness and abundance. Journal of Tropical Ecology, 25, 107-121.

Wood, S.N. (2011) Fast stable restricted maximum likelihood and marginal likelihood estimation of semiparametric generalized linear models. Journal of the Royal Statistical Society B: Statistical Methodology, 73, 3-36.

Woods, C.L. (2013) Factors influencing the distribution and structure of tropical vascular epiphyte communities at multiple scales. PhD Thesis, Clemson University, South Carolina. 
Woods, C.L., Cardelús, C.L. \& Dewalt, S.J. (2015) Microhabitat associations of vascular epiphytes in a wet tropical forest canopy. Journal of Ecology, 103, 421-430.

Worbes, M., Staschel, R., Roloff, A. \& Junk, W.J. (2003) Tree ring analysis reveals age structure, dynamics and wood production of a natural forest stand in Cameroon. Forest Ecology and Management, 173, 105-123.

Wright, S.J. (2005) Tropical forests in a changing environment. Trends in Ecology and Evolution, 20, 553-560.

Wright, I.J. \& Cannon, K. (2001) Relationships between leaf lifespan and structural defenses in a low nutrient, schlerophyll flora. Functional Ecology, 15, 351-359.

Wright, I.J., Falster, D.S., Pickup, M. \& Westoby, M. (2006) Cross-species patterns in the coordination between leaf and stem traits, and their implications for plant hydraulics. Physiologia Plantarum, 127, 445-456.

Wright, S.J., Horlyck, V., Basset, Y., Barrios, H., Bethancourt, A., Bohlman, S., Gilbert, G., Goldstein, G., Graham, E.A., Kitajima, K., Lerdau, M.T., Meinzer, F.C., Ødegaard, F., Reynolds, D.R., Roubik, D.W., Sakai, S., Samaniego, M., Sparks, J.P., Van Bael, S., Winter, K. \& Zotz, G. (2003a) Tropical Canopy Biology Program, Republic of Panama. Studying Forest Canopies from Above: The International Canopy Crane Network (eds Y. Basset, V. Horlyck \& S.J. Wright), pp. 137-155. Smithsonian Tropical Research Institute, New York.

Wright, S.J., Kitajima, K., Kraft, N.J.B., Reich, P.B., Wright, I.J., Bunker, D.E., Condit, R., Dalling, J.W., Davies, S.J., Díaz, S., Engelbrecht, B.M.J., Harms, K.E., Hubbell, S.P., Marks, C.O., Ruiz-Jaen, M.C., Salvador, C.M. \& Zanne, A.E. (2010) Functional traits and the growth-mortality trade-off in tropical trees. Ecology, 91, 366474.

Wright, S.J., Muller-Landau, H.C., Condit, R. \& Hubbell, S.P. (2003b) Gap-dependent recruitment, realised vital rates, and size distributions of tropical trees. Ecology, 84, 3174-3185.

Wright, I.J., Reich, P.B., Cornelissen, J.H.C., Falster, D.S., Garnier, E., Hikosaka, K., Lamont, B.B., Lee, W., Oleksyn, J., Osada, N., Poorter, H., Villar, R., Warton, D.I. \& Westoby, M. (2005a) Assessing the generality of global leaf trait relationships. New Phytologist, 166, 485-496.

Wright, I.J., Reich, P.B., Cornelissen, J.H.C., Falster, D.S., Groom, P.K., Hikosaka, K., Lee, W., Lusk, C.H., Niinemets, Ü., Oleksyn, J., Osada, N., Poorter, H., Warton, D.I. \& Westoby, M. (2005b) Modulation of leaf economic traits and trait relationships by climate. Global Ecology and Biogeography, 14, 411-421.

Wright, I.J., Reich, P.B., Westoby, M., Ackerly, D.D., Baruch, Z., Bongers, F., Cavender-Bares, J., Chapin, T., Cornelissen, J.H.C., Diemer, M., Flexas, J., Garnier, E., Groom, P.K., Gulias, J., Hikosaka, K., Lamont, B.B., Lee, T., Lee, W., Lusk, C., Midgley, J.J., Navas, M.-L., Niinemets, U., Oleksyn, J., Osada, N., Poorter, H., Poot, P., Prior, L., Pyankov, V.I., Roumet, C., Thomas, S.C., Tjoelker, M.G., Veneklaas, E.J. \& Villar, R. (2004) The worldwide leaf economics spectrum. Nature, 428, 821-827.

Yanoviak, S.P., Nadkarni, N.M. \& Solano J., R. (2007) Arthropod assemblages in epiphyte mats of Costa Rican cloud forests. Biotropica, 39, 202-210.

Zotz, G. (1998) Demography of the epiphytic orchid, Dimerandra emarginata. Journal of Tropical Ecology, 14, 725741.

Zotz, G. (2000) Size-related intraspecific variability in physiological traits of vascular epiphytes and its importance for plant physiological ecology. Perspectives in Plant Ecology, Evolution and Systematics, 3, 19-28.

Zotz, G. (2004) How prevalent is crassulacean acid metabolism among vascular epiphytes? Oecologia, 138, 184-92.

Zotz, G. (2005) Vascular epiphytes in the temperate zones - a review. Plant Ecology, 176, 173-183.

Zotz, G. (2007) Johansson revisited: the spatial structure of epiphyte assemblages. Journal of Vegetation Science, 18, $123-130$. 


\section{References}

Zotz, G. (2013) The systematic distribution of vascular epiphytes - a critical update. Botanical Journal of the Linnean Society, 171, 453-481.

Zotz, G. \& Asshoff, R. (2010) Growth in epiphytic bromeliads: response to the relative supply of phosphorus and nitrogen. Plant Biology, 12, 108-113.

Zotz, G. \& Bader, M.Y. (2011) Sampling vascular epiphyte diversity - species richness and community structure. Ecotropica, 17, 103-112.

Zotz, G., Bermejo, P. \& Dietz, H. (1999) The epiphyte vegetation of Annona glabra on Barro Colorado Island, Panama. Journal of Biogeography, 26, 761-776.

Zotz, G. \& Hietz, P. (2001) The physiological ecology of vascular epiphytes: current knowledge, open questions. Journal of Experimental Botany, 52, 2067-2078.

Zotz, G., Laube, S. \& Schmidt, G. (2005) Long-term population dynamics of the epiphytic bromeliad, Werauhia sanguinolenta. Ecography, 28, 806-814.

Zotz, G. \& Schmidt, G. (2006) Population decline in the epiphytic orchid Aspasia principissa. Biological Conservation, 129, 82-90.

Zotz, G. \& Schultz, S. (2008) The vascular epiphytes of a lowland forest in Panama - species composition and spatial structure. Plant Ecology, 195, 131-141.

Zotz, G. \& Vollrath, B. (2003) The epiphyte vegetation of the palm Socratea exorrhiza - correlations with tree size, tree age and bryophyte cover. Journal of Tropical Ecology, 19, 81-90.

Zuur, A.F., Ieno, E.N., Walker, N.J., Saveliev, A.A. \& Smith, G.M. (2009) Mixed Effects Models and Extensions in Ecology with R. Springer (eds M. Gail, K. Krickeberg, J.M. Samet, A. Tsiatis, and W. Wong). New York, USA. 


\section{Appendix}




\section{A Supplementary information to chapter 2}
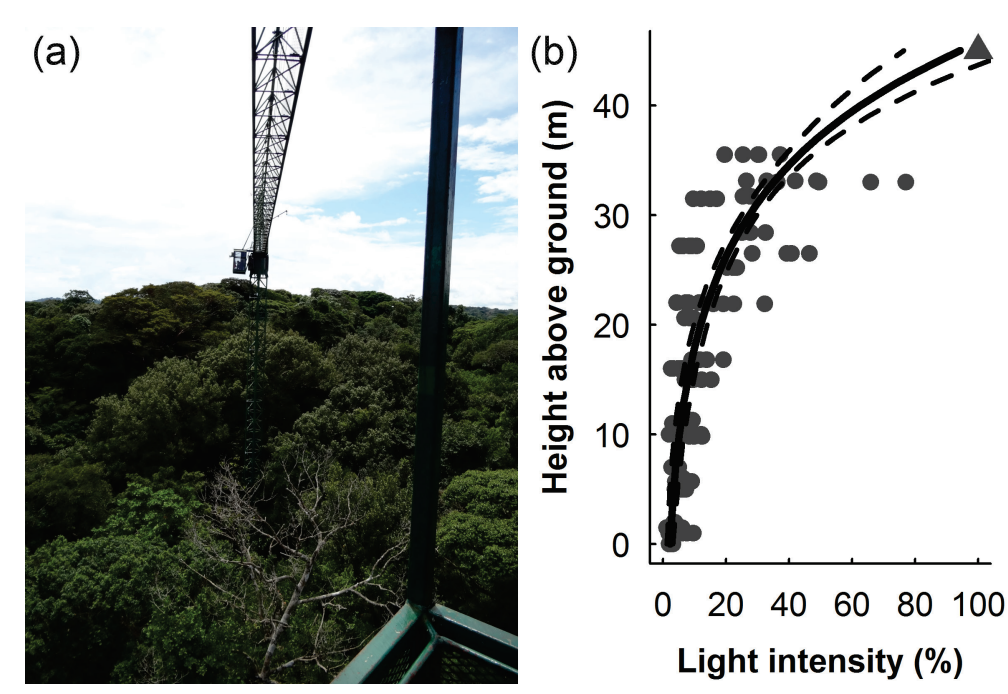

Light intensity (\%)

Figure A.1. (a) Photograph of the study site (San Lorenzo Canopy Crane site) at the Atlantic coast of Panama. Canopy height in this undisturbed lowland rainforest is variable, with a few emergent trees reaching a maximum height of ca. $45 \mathrm{~m}$. A small gondola attached to a construction crane (height: $52 \mathrm{~m}$; radial length: $54 \mathrm{~m}$ ) allows access to all strata of the forest within an area of ca. 0.9 ha. (b) Vertical light intensity gradient at the study site. Light intensity was estimated using Hobo data loggers (HOBO UA-002-64; Onset Computer Corporation, Cape Cod, USA), which were mounted at different heights in three trees and above the canopy (the grey triangle at $45 \mathrm{~m}$ represents the maximum canopy height). Light intensity was logged every minute over 6-8 days in the late rainy season in 2012. Daily sums of light intensity were calculated and, on this basis, the within-canopy measurements were related to the measurement above the canopy. Non-linear and linear regression models were fitted and compared by AIC. The light intensity gradient (solid line) was best approximated by the Lambert-Beer light attenuation model $\mathrm{I}=\mathrm{I} 0^{*} \mathrm{e}^{-(\mathrm{k} * \mathrm{LAI})}$, with an attenuation coefficient of $\mathrm{k}=0.5$ and a leaf area index of LAI=7.5. Dashed lines indicate $95 \%$ confidence interval. 

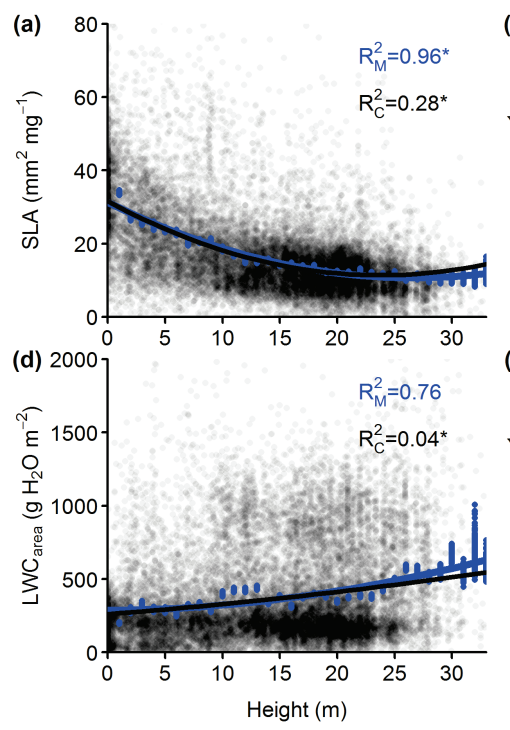
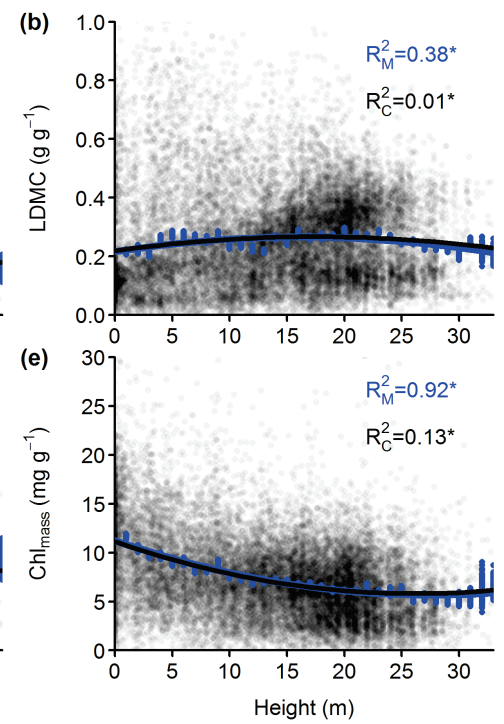
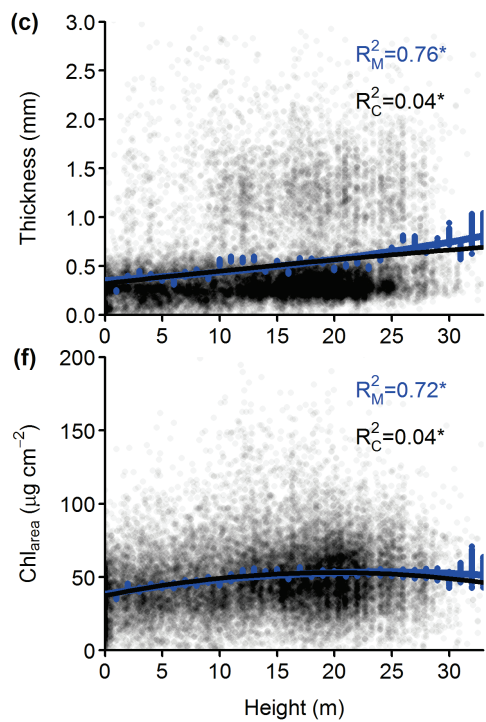

Figure A.2. Relationship between height above ground and six leaf trait: (a) specific leaf area (SLA), (b) leaf dry matter content (LDMC), (c) leaf thickness, (d) leaf water content ( $\mathrm{LWC}_{\text {area }}$ ), (e) leaf chlorophyll content on mass basis $\left(\mathrm{Ch} l_{\text {mass }}\right)$, (f) leaf chlorophyll content on area basis ( $\left.\mathrm{Chl}_{\text {area }}\right)$. Blue dots represent community mean trait values calculated for all 1-m height intervals, black dots represent (predicted) trait values of all individuals (see below). $\mathrm{R}^{2} \mathrm{M}$ : amount of variance in community mean trait values explained by height. $\mathrm{R}^{2} \mathrm{C}$ : amount of variance in individuals' trait values explained by height. In contrast to the analyses in the main manuscript, these trait-height relationships are based on the height distribution and species identity of all individuals recorded in a comprehensive census at the study site ( $>22,000$ individuals of $>100$ species; Glenda Mendieta-Leiva \& Gerhard Zotz, unpublished data; see Zotz \& Schultz 2008 for methodology). To this end, we predicted trait values for each individual epiphyte recorded in the census based on its observed height and its intraspecific trait-height relationships (see Figs A.4-A.9). More specifically, trait values were randomly chosen from the $95 \%$ prediction intervals of the intraspecific trait-height relationship assuming normally distributed probability densities (Figs A.4-A.9). Community mean trait values for 1-m height intervals were then calculated by averaging predicted trait values over all individuals within each interval. The entire procedure was repeated 100 times to account for the stochasticity involved in choosing trait values from prediction intervals. As intraspecific trait-height relationships might be inaccurate if the number of samples is too small, we only considered species with $\geq 10$ records per trait. This excluded the NC traits from analysis, but covered $86 \%$ of all individuals in the census for the extensively sampled traits as our sampling design included the most abundant species. While the uncertainty associated with the use of prediction intervals of intraspecific trait-height relationships is a weakness of this procedure, it is an advantage that the uneven distribution of the species and their true abundance are accounted for. Some species were much more abundant than others (e.g. Ananthacorus angustifolius with $>2,300$ individuals and Scaphyglottis longicaulis with $>1,900$ individuals) and due to our sampling strategy, it was not feasible to exactly represent their relative abundances in our sub-sample. Community trait means might be driven by few highly abundant species, and thus we regarded the procedure used here as indicator whether trait-height relationships analyzed based on the sub-sample (see main manuscript) might be biased due to our sampling strategy. This would be the case if traitheight relationships differed substantially (compare this figure and Fig. 2.1, as well as Table A.3 and A.4). However, as there are only slight differences, we conclude that trait-height relationships were not substantially biased due to our sampling strategy. 

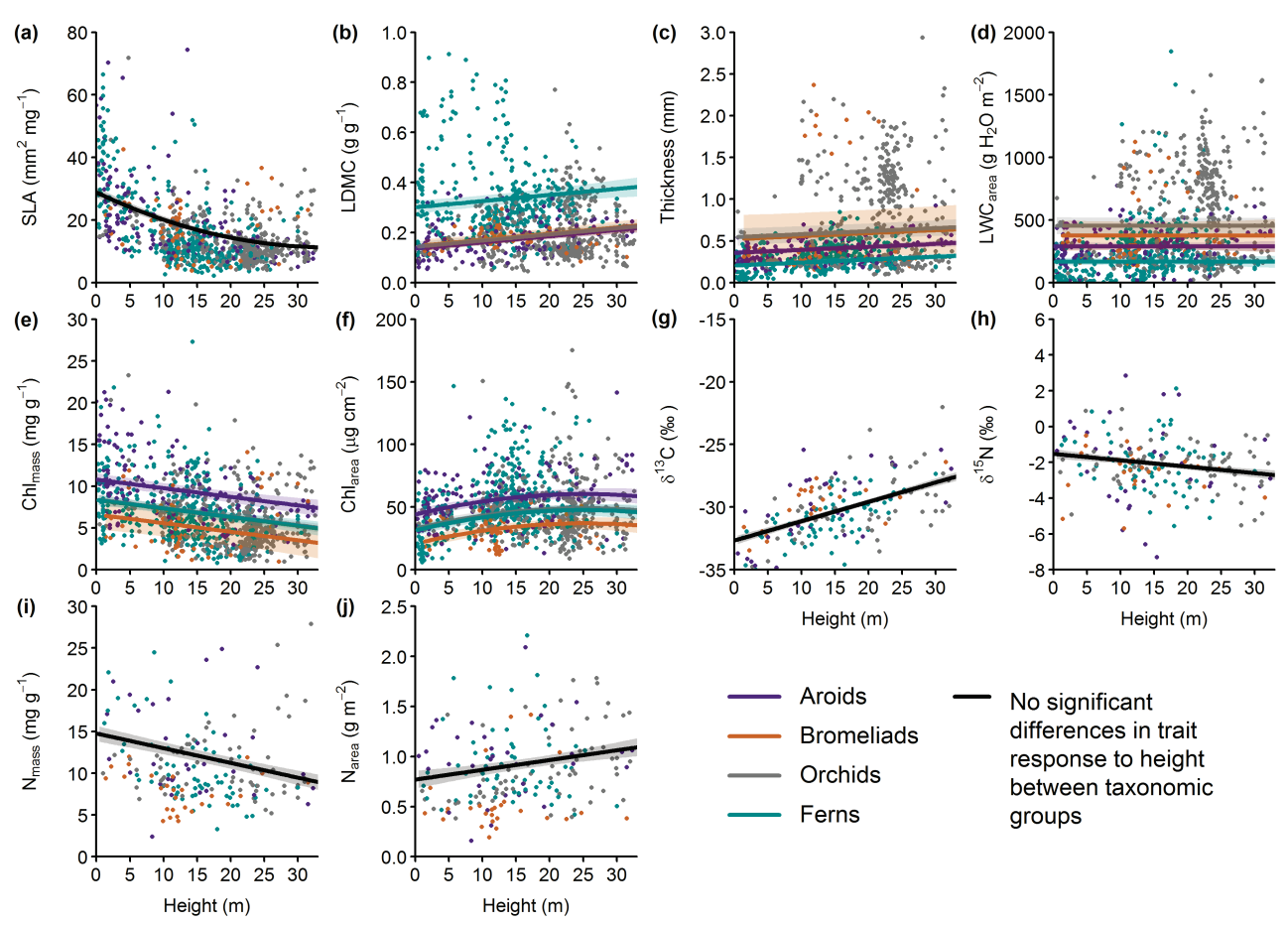

Figure A.3. Trait-height relationships for four taxonomic groups of vascular epiphytes (aroids, bromeliads, orchids and ferns): (a) SLA: specific leaf area, (b) LDMC: leaf dry matter content, (c) Thickness: leaf thickness, (d) LWC area: leaf water content per leaf area, (e) Chlmass: mass-based chlorophyll concentration, (f) Chlarea: area-based chlorophyll concentration, (g) $\delta^{13} \mathrm{C}$ : carbon isotope ratio, (h) $\delta^{15} \mathrm{~N}$ : nitrogen isotope ratio, (i) $\mathrm{N}_{\text {mass }}$ : mass-based nitrogen concentration, (j) $\mathrm{N}_{\text {area }}$ : area-based nitrogen concentration. The minimal adequate models indicating between-group differences are shown. The colored shaded areas indicate $95 \%$ confidence intervals. Differences were analyzed using generalized linear mixed models (GLMMs; R package 'nlme'; dependent variable: trait; fixed effects: height, height ${ }^{2}$, taxonomic group; random effect: species; see Table A.2for details). We fitted the GLMMs with full fixed effects and all possible combinations of random effects (Table A.2) to trait data to obtain the minimal adequate random structure using the REML estimation method (Zuur et al. 2009). Using the minimal adequate random structure for each trait, we compared GLMMs with all meaningful fixed-effect combinations (Table A.2) by applying the ML estimation method to obtain the MAM. Simpler models were preferred to more complex when $\triangle$ AIC $\leq 10$ (Burnham \& Anderson 2004). When the fixed-effect structure of the MAM included the interaction between height and taxonomic groups, trait-height relationships significantly differed between taxonomic groups. We used GLMMs here because they control for difference in abundance among species, meaning that they indicate the average trend of species within their taxonomic groups. In contrast to the max- $t$ test used to compare the trait means between taxonomic groups (Table 2.1 in main manuscript), the GLMMs are suitable to further indicate whether possible differences in traits means are still significant after controlling for the effect of height and whether trait responses, in general, differ between groups. Note that no differences in leaf trait responses to height among taxonomic groups were observed. This was striking, as we hypothesized that taxonomically conserved differences in morphological or physiological characteristics might also affect how leaf traits respond to environmental changes with height. For instance, the water- and nutrient-storing pseudobulbs in orchids might decrease the necessity of their leaves to adjust to drier conditions. In contrast, ferns depend to a larger degree on their leaves to control water balance, and thus we expected that ferns might be more dependent on adjustments of their leaves. However, the observed lack of differences in trait response might also be related to the fact that taxonomic groups can be quite heterogeneous, considering that, for example, orchid species can have deciduous leaves (e.g. Catasetinae orchids) as well as pseudobulbs of different sizes, or even none. Therefore, species-specific characteristics per se might be more important for the trait response of species than its broader taxonomic affiliation. Interestingly, SLA was the single extensively sampled trait for which no significant difference in slopes or intercepts were observed (a). This pattern suggests an optimal SLA value at a given height independent of taxonomic group, and furthermore indicates that the community trend is both influenced by the turnover of species differing in mean SLA values as well as their intraspecific response to height (Fig. A.4). The fact that no differences in slopes or intercepts were observed for NC traits $(g-j)$ should not be over-interpreted. For these traits, the differences in AIC values between most models were below the chosen threshold $(\triangle \mathrm{AIC}=10$; Burnham \& Anderson 2004), and therefore the simplest model using only height as fixed effect was preferred. This is likely due to lower sample sizes for NC traits, which needs to be increased for detailed interpretations. 


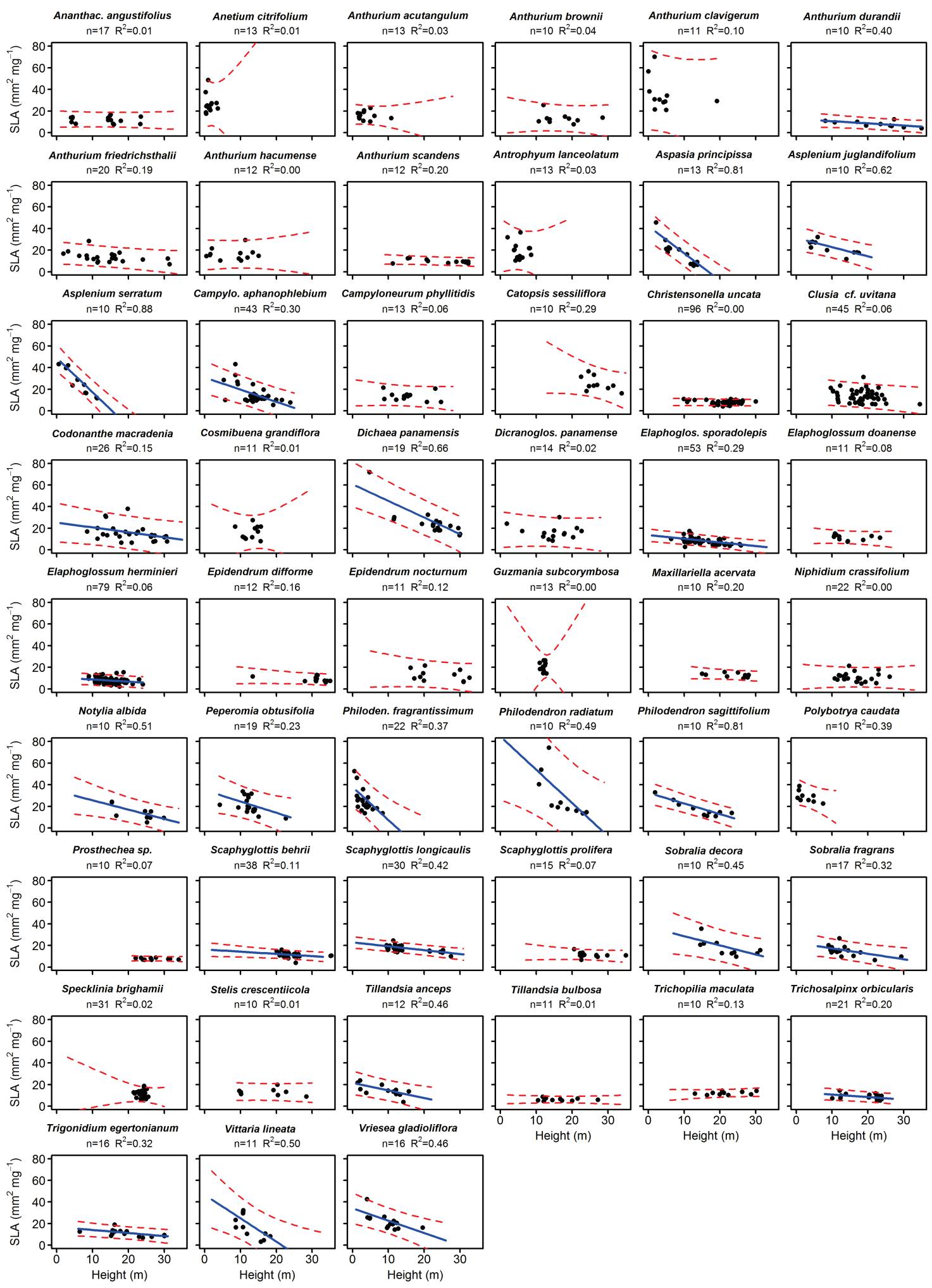

Figure A.4. Intraspecific trait-height relationship between specific leaf area (SLA) and height above ground for all species with $\geq 10$ individuals sampled. Blue regression lines indicate significant correlations $(P<0.05)$, red dashed lines indicate $95 \%$ prediction intervals. Prediction intervals are shown only for the realized height distribution of the particular species as recorded in the comprehensive census. 

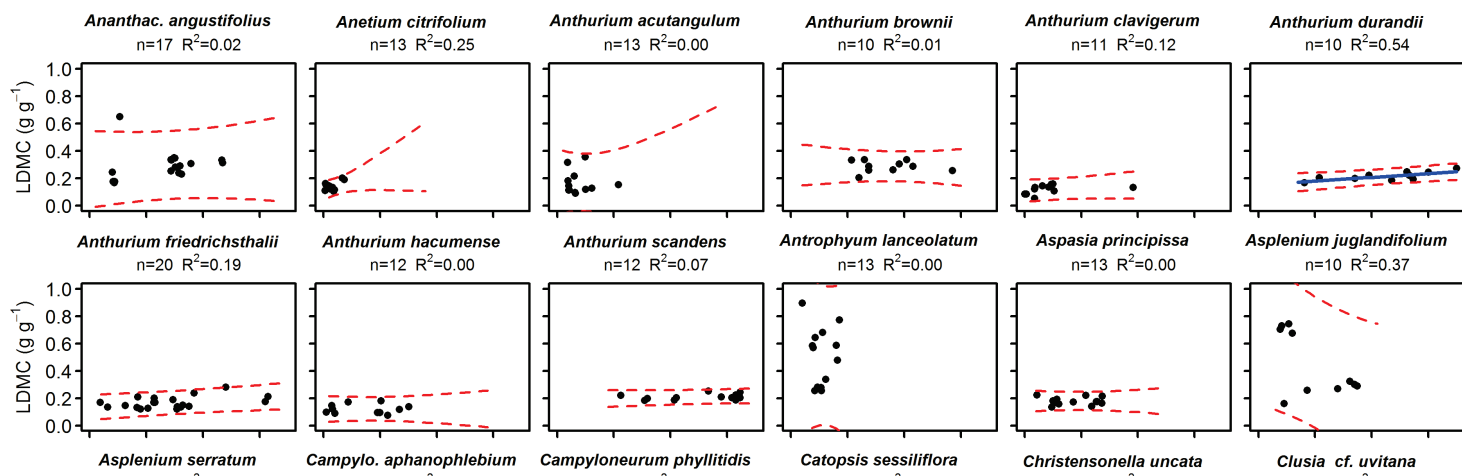

$n=12 \quad R^{2}=0.07$
$n$

Antrophyum lanceolatum

Aspasia principissa
$n=13 \quad R^{2}=0.00$

Asplenium juglandifolium
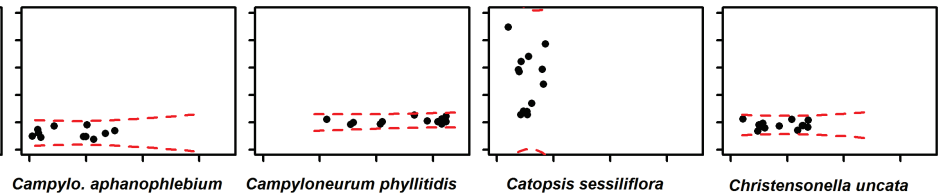

Catopsis sessiliflora $n=10 \quad R^{2}=0.10$

Christensonella uncata $n=96 \quad R^{2}=0.01$

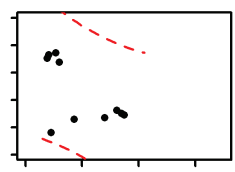
$n=13 R^{2}=0.33$
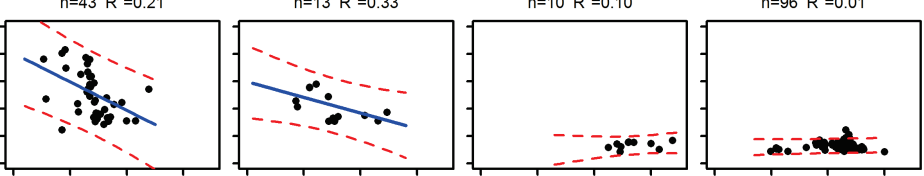

Clusia cf. uvitan $n=45 \quad R^{2}=0.13$

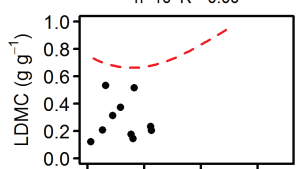

Cosmibuena grandiflora

\section{Dichaea panamensis}

Dicranoglos. panamense

Elaphoglos. sporadolepis

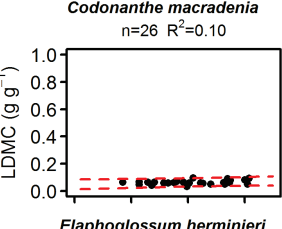

$n=11 R^{2}=0.06$
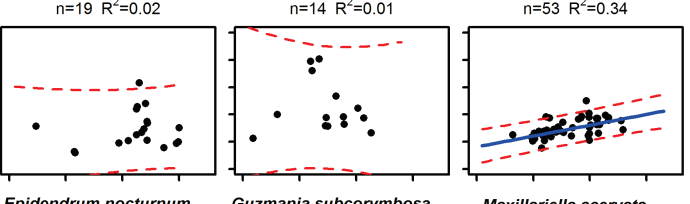

$n=79 R^{2}=0.05$

Epidendrum difforme

$n=11 \quad R^{2}=0.25$

Guzmania subcorymbosa

$n=13 R^{2}=0.00$

Maxillariella acervata
$\mathrm{n}=10 \quad \mathrm{R}^{2}=0.74$
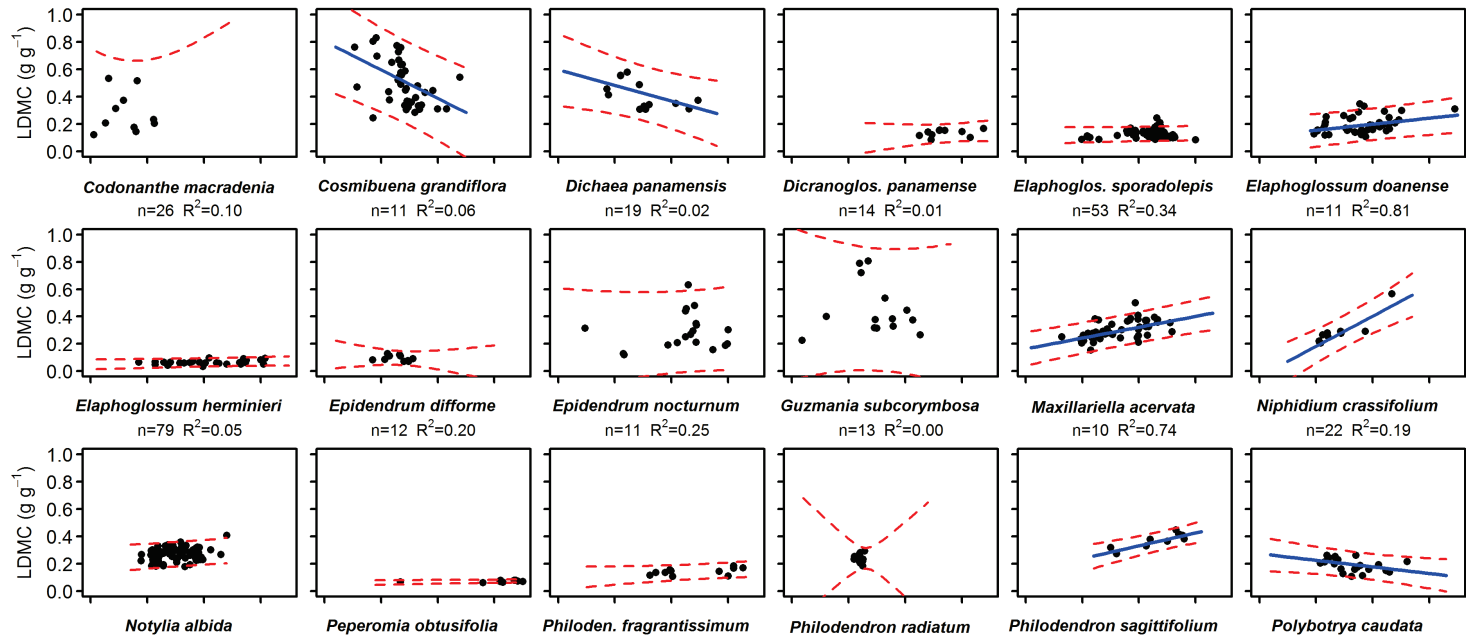

Peperomia obtusifolia

Philoden. fragrantissimum $n=22 \quad R^{2}=0.37$

Philodendron radiatum $n=10 \quad R^{2}=0.49$

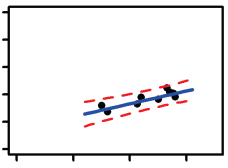

Elaphoglossum doanense
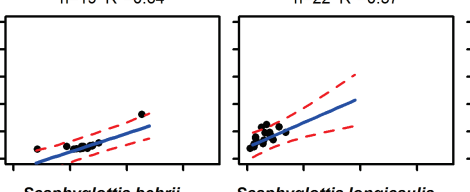

$n=38 R^{2}=0.06$
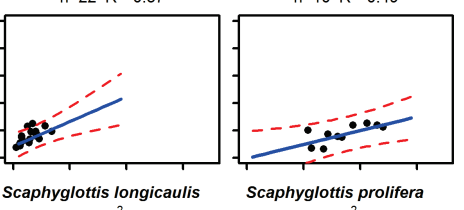

Philodendron sagittifolium $n=10 \quad R^{2}=0.62$

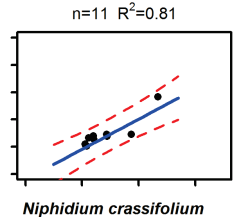

iphidium crassifolium
$n=22 R^{2}=0.19$

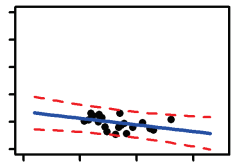

Polybotrya caudata

$n=10 \quad R^{2}=0.08$
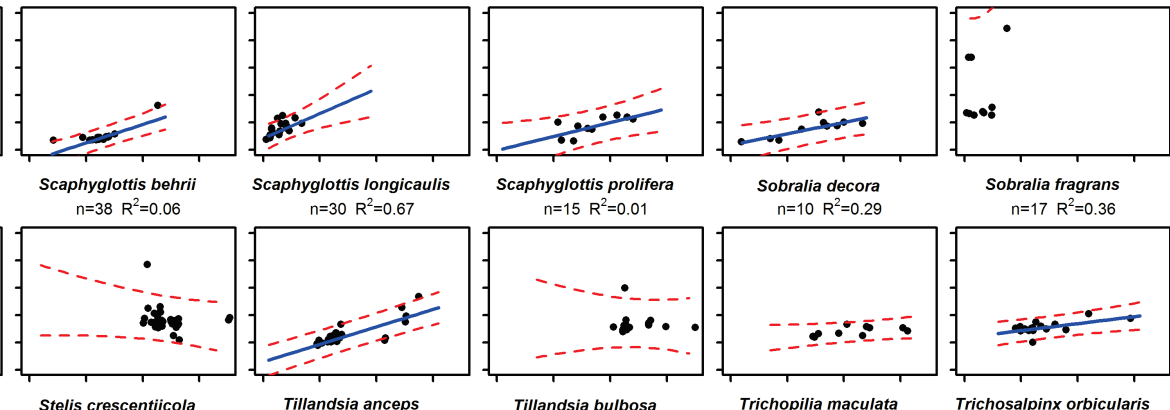

$n=30 \quad R^{2}=0.67$

Scaphyglottis prolifera

$n=10 \quad R^{2}=0.29$

Sobralia fragrans

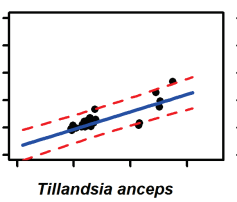

$n=15 \quad R^{2}=0.01$

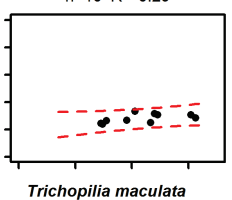

$n=17 R^{2}=0.36$

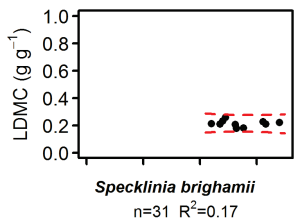

Stelis crescentiicola
$n=10 \quad R^{2}=0.11$

$\mathrm{n}=12 \quad \mathrm{R}^{2}=0.39$

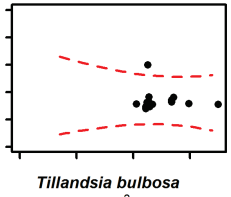

$\mathrm{n}=10 \quad \mathrm{R}^{2}=0.00$
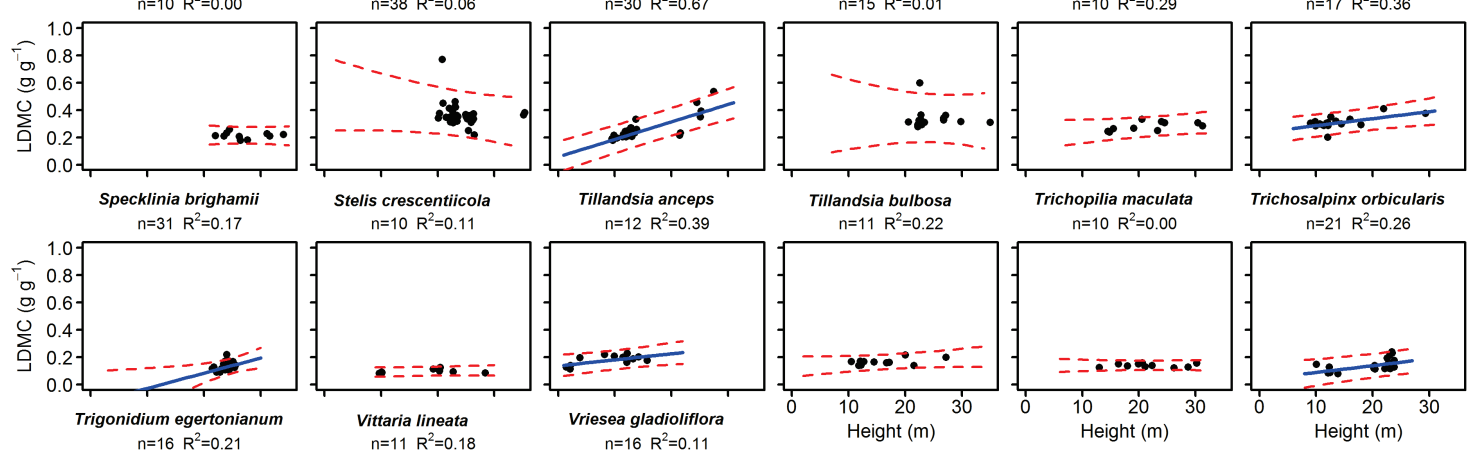

Trichosalpinx orbicularis
$n=21 \quad R^{2}=0.26$
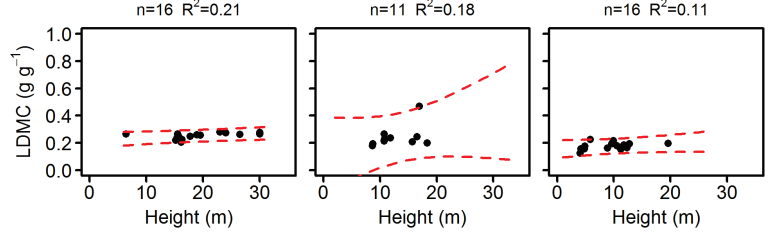

Figure A.5. Intraspecific trait-height relationship between leaf dry mass content (LDMC) and height above ground for all species with $\geq 10$ individuals sampled. Blue regression lines indicate significant correlations $(P<0.05)$, red dashed lines indicate $95 \%$ prediction intervals. Prediction intervals are shown only for the realized height distribution of the particular species as recorded in the comprehensive census. 


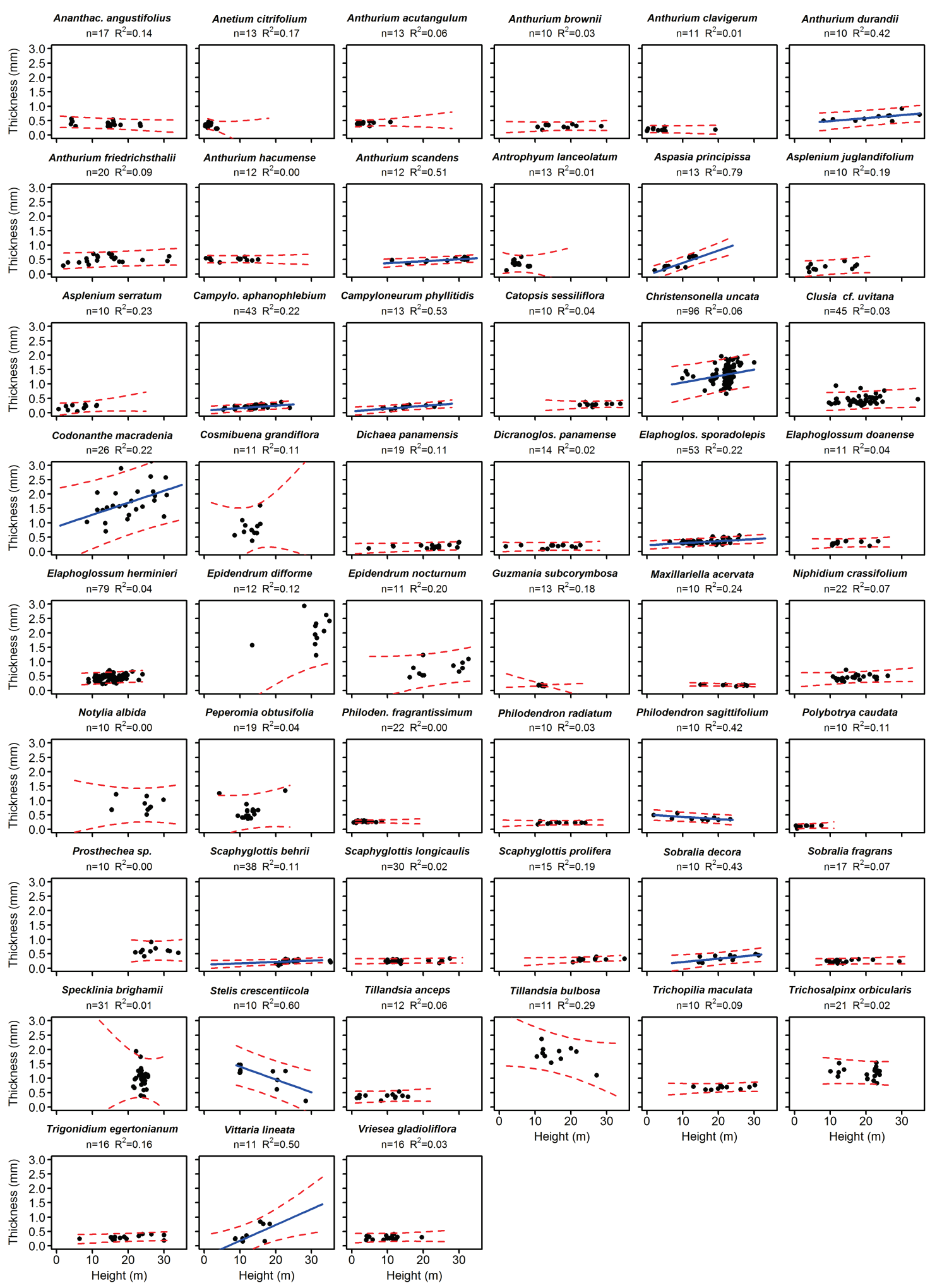

Figure A.6. Intraspecific trait-height relationship between leaf thickness and height above ground for all species with $\geq 10$ individuals sampled. Blue regression lines indicate significant correlations $(P<0.05)$, red dashed lines indicate $95 \%$ prediction intervals. Prediction intervals are shown only for the realized height distribution of the particular species as recorded in the comprehensive census. 


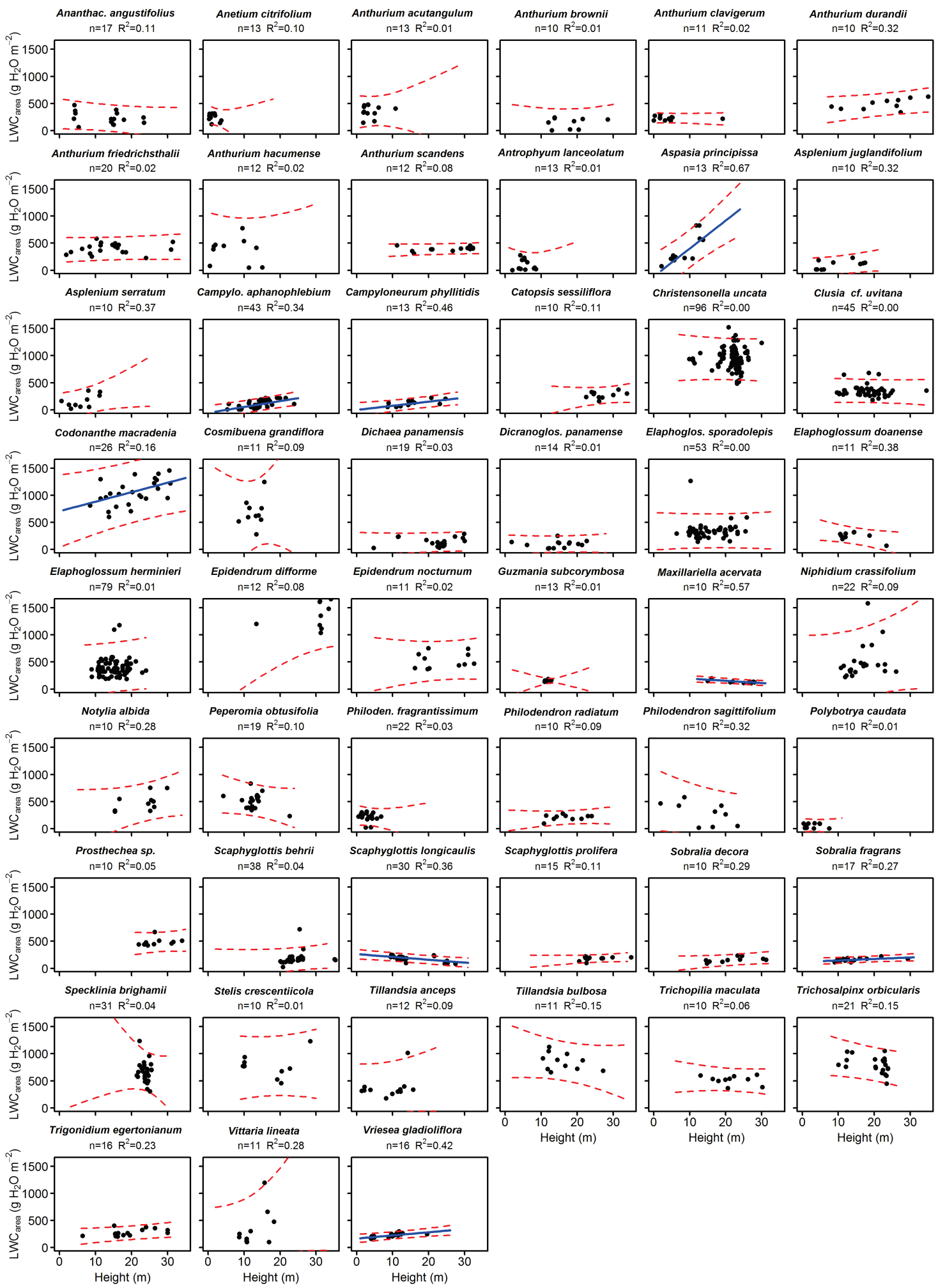

Figure A.7. Intraspecific trait-height relationship between leaf water content ( $\left.\mathrm{LWC}_{\text {area }}\right)$ and height above ground for all species with $\geq 10$ individuals sampled. Blue regression lines indicate significant correlations $(P<0.05)$, red dashed lines indicate $95 \%$ prediction intervals. Prediction intervals are shown only for the realized height distribution of the particular species as recorded in the comprehensive census. 


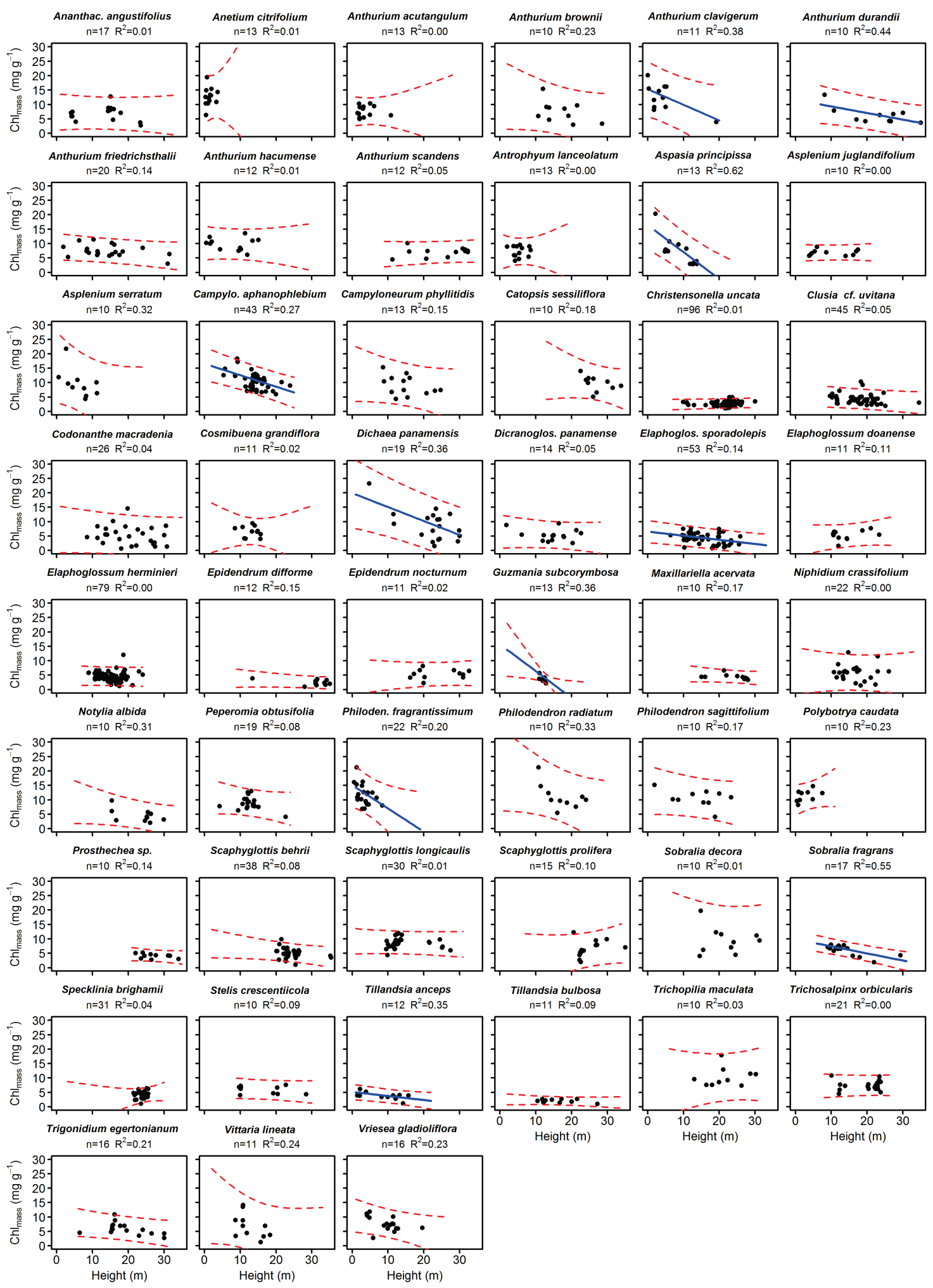

Figure A.8. Intraspecific trait-height relationship between leaf chlorophyll content per leaf dry mass (Chl mass) and height above ground for all species with $\geq 10$ individuals sampled. Blue regression lines indicate significant correlations $(P<0.05)$, red dashed lines indicate $95 \%$ prediction intervals. Prediction intervals are shown only for the realized height distribution of the particular species as recorded in the comprehensive census. 


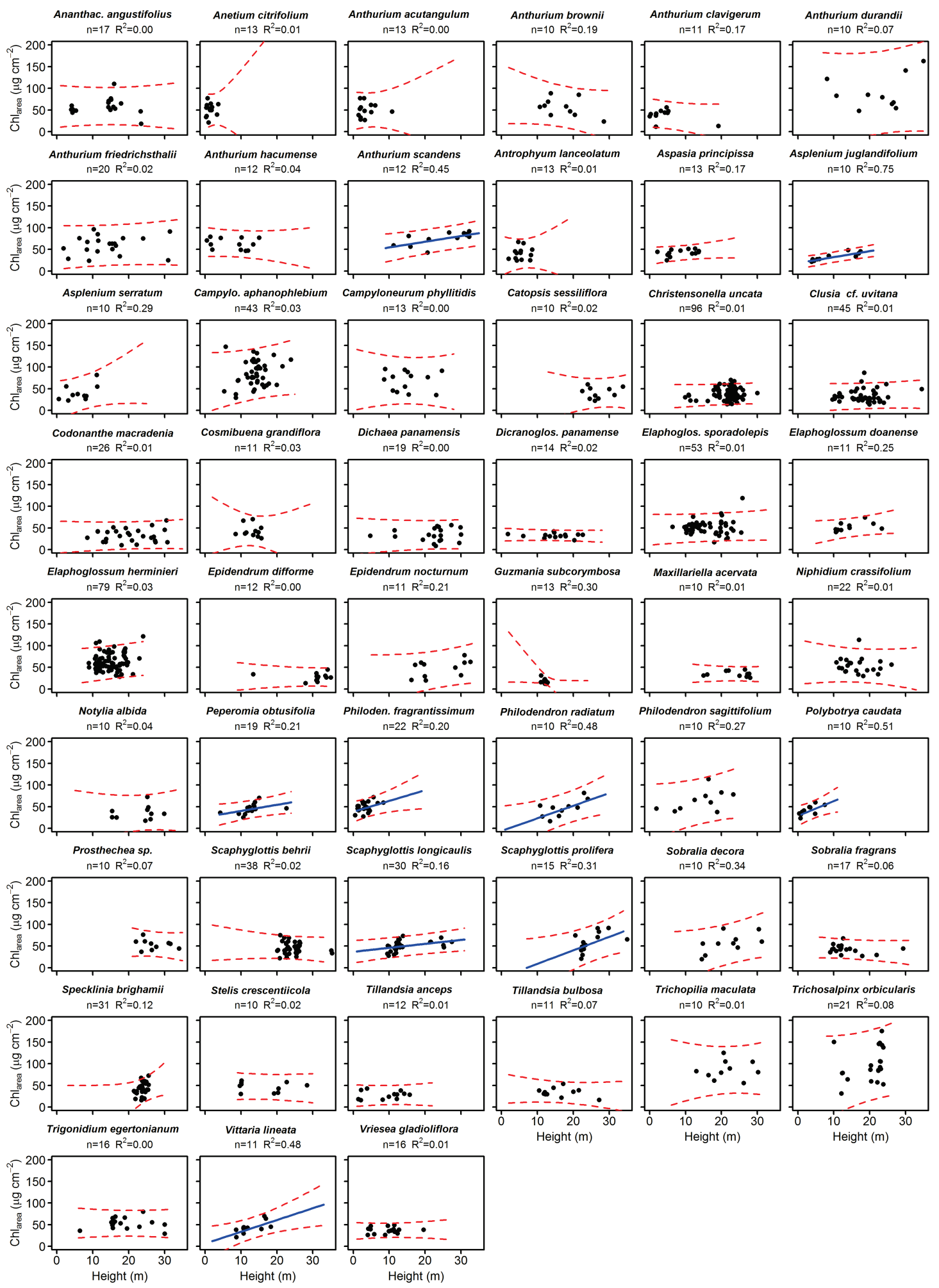

Figure A.9. Intraspecific trait-height relationship between leaf chlorophyll content per leaf area $\left(\mathrm{Chl}_{\text {area }}\right)$ and height above ground for all species with $\geq 10$ individuals sampled. Blue regression lines indicate significant correlations $(\mathrm{P}<0.05)$, red dashed lines indicate $95 \%$ prediction intervals. Prediction intervals are shown only for the realized height distribution of the particular species as recorded in the comprehensive census. 


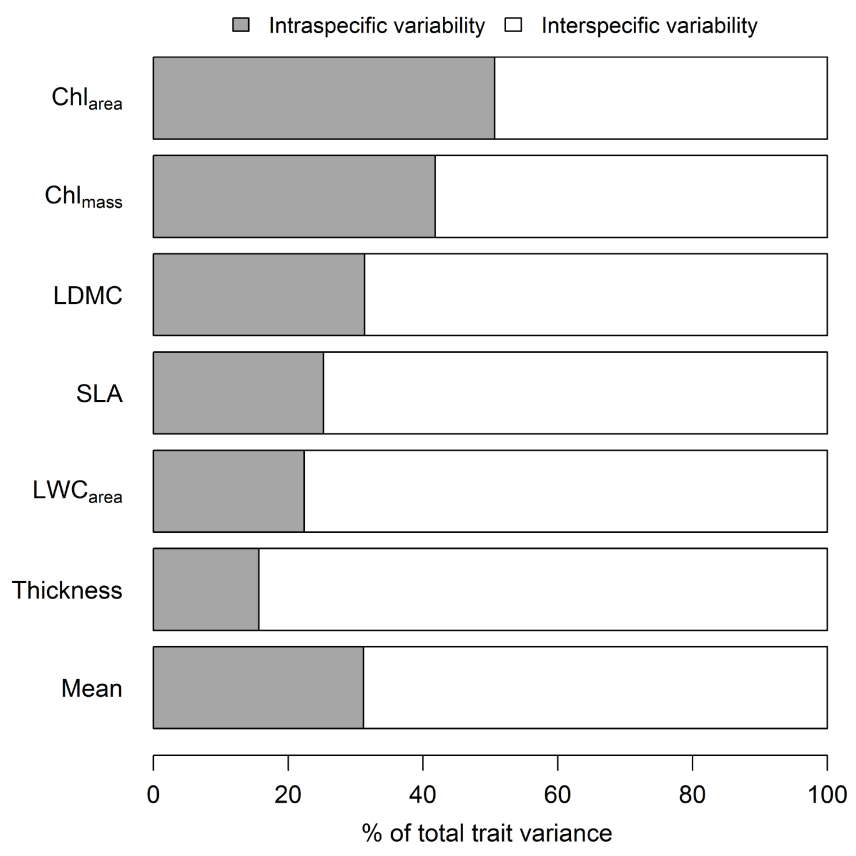

Figure A.10. Partitioning of total trait variance into between-species (interspecific) variance and within-species (intraspecific) variance for six leaf traits (SLA: specific leaf area, LDMC: leaf dry matter content, Thickness: leaf thickness, $\mathrm{LWC}_{\text {area }}$ : leaf water content per leaf area, $\mathrm{Chl}_{\text {mass: }}$ : mass-based leaf chlorophyll concentration, Chl area: areabased leaf chlorophyll concentration). We only considered species with $\geq 10$ records per trait here.

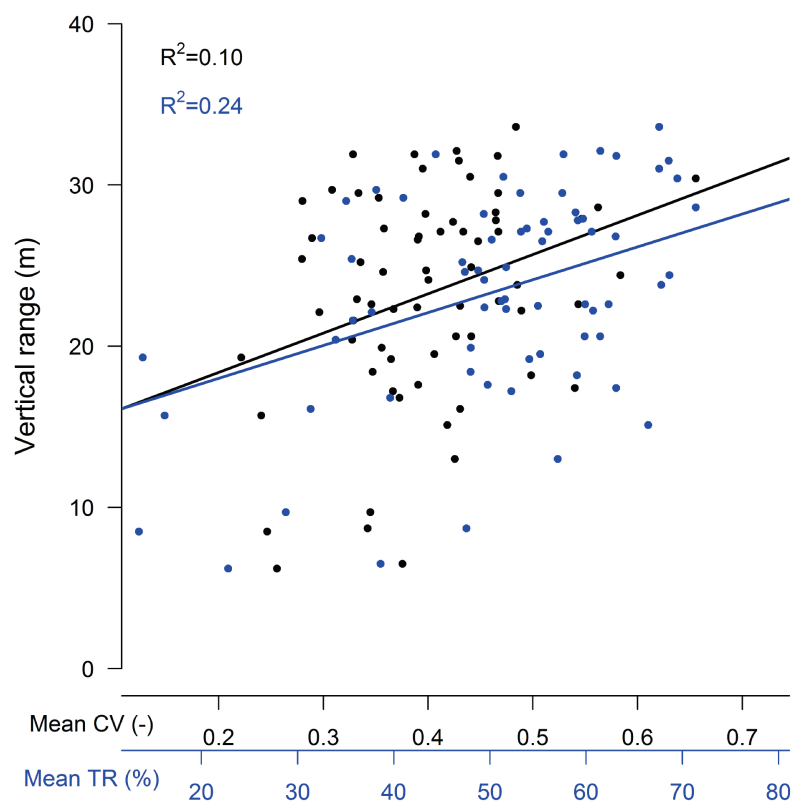

Figure A.11. Relationship between intraspecific trait variability and vertical range of epiphyte species in a Panamanian lowland forest based on a linear model (vertical range $\sim$ trait variability). The vertical range of each species was estimated based on its height distribution in the extensive census conducted in 2010-2012 (Glenda Mendieta-Leiva \& Gerhard Zotz, unpublished data; see Zotz \& Schultz 2008 for methodology). Two measures of trait variability for each species and trait were calculated: the coefficient of variation $(\mathrm{CV})$ and the trait range (TR: absolute difference between maximum and minimum trait value divided by the maximum, given in \%). The mean CV and mean TR over all traits were used as measures of multivariate intraspecific trait variability and are shown here. $\mathrm{R}^{2}$ : amount of variance in vertical range explained by $\mathrm{CV}$ or $\mathrm{TR}$. We only considered species with $\geq 10$ records per trait. 
Supplementary information - chapter 2

Table A.1. Mean leaf trait values \pm SD of 83 epiphyte species recorded in a Panamanian lowland forest. Height above the ground was measured for each individual plant. Some leaf traits (Thickness, SLA, LWC area, LDMC, Chl area, Chl mass) were sampled more extensively: $\mathrm{n}$ (field). For a subset, $\mathrm{N}_{\text {mass }}, \mathrm{N}_{\text {area, }}, \delta^{13} \mathrm{C}$ and $\delta^{15} \mathrm{~N}$ were determined in a laboratory: $\mathrm{n}$ (lab). Species names follow The Plant List (http://www.theplantlist.org). Photosynthetic pathway was derived from carbon isotope ratios $\delta^{13} \mathrm{C}$, with values $>-20 \%$ indicating CAM metabolism. All ferns are marked with (F). Mean CV: mean coefficient of variation over extensively sampled traits. Mean TR: mean trait range over extensively sampled traits. At the end of the table, mean trait values over all species, as well as reported global trait means of non-epiphytic taxa (TRY - a global database of plant traits; Kattge et al. 2011) are given.

\begin{tabular}{|c|c|c|c|c|c|c|c|c|c|c|c|c|c|c|c|c|}
\hline Species & Family & \begin{tabular}{|l|} 
Phot. \\
Pathway
\end{tabular} & $\begin{array}{l}\mathrm{n} \\
\text { (field) }\end{array}$ & $\begin{array}{l}\text { SLA } \\
\left(\mathrm{mm}^{2} \mathrm{mg}^{-1}\right)\end{array}$ & $\begin{array}{l}\text { LDMC } \\
\left(\mathrm{g} \mathrm{g}^{-1}\right)\end{array}$ & $\begin{array}{l}\text { Thickness } \\
(\mathrm{mm})\end{array}$ & $\begin{array}{l}\begin{array}{l}\mathbf{L W C} \text { area } \\
\left(\mathrm{g} \mathrm{H}_{2} \mathrm{O} \mathbf{~ m}^{-2}\right)\end{array} \\
\end{array}$ & $\begin{array}{l}\begin{array}{l}\text { Chl mass } \\
\left(\mathrm{mg} \mathrm{g}^{-1}\right)\end{array} \\
\end{array}$ & $\begin{array}{l}\begin{array}{l}\text { Chl } \\
\left(\mu \mathrm{a} \mathrm{cm}^{-2}\right)\end{array} \\
\end{array}$ & $\begin{array}{l}\text { Mean CV } \\
(-)\end{array}$ & $\begin{array}{l}\begin{array}{l}\text { Mean TR } \\
(\%)\end{array} \\
\end{array}$ & $\begin{array}{l}\mathbf{n} \\
\text { (lab) }\end{array}$ & $\begin{array}{l}\delta^{13} \mathrm{C} \\
(\%)\end{array}$ & $\begin{array}{l}\delta^{15} \mathrm{~N} \\
(\%)\end{array}$ & $\begin{array}{l}\mathbf{N}_{\text {mass }} \\
\left(\mathbf{m g ~ g}^{-1}\right)\end{array}$ & $\begin{array}{l}N_{\text {area }} \\
\left(\mathrm{g} \mathrm{m}^{-2}\right)\end{array}$ \\
\hline Acianthera verecunda & Orchidaceae & CAM & 7 & $15.7 \pm 5.9$ & $0.07 \pm 0.02$ & $1.04 \pm 0.28$ & $993 \pm 354$ & $4.8 \pm 2.7$ & $29.6 \pm 8.5$ & 0.44 & 51.7 & 3 & $-13.2 \pm 1.2$ & $-1.5 \pm 1.1$ & $11.2 \pm 4.6$ & $0.77 \pm 0.21$ \\
\hline Ananthacorus angustifolius & Pteridaceae (F) & C3 & 17 & $12.0 \pm 3.0$ & $0.30 \pm 0.11$ & $0.39 \pm 0.08$ & $233 \pm 113$ & $7.0 \pm 2.4$ & $58.9 \pm 19.0$ & 0.43 & 61.5 & 3 & $-31.9 \pm 0.5$ & $-2.2 \pm 0.6$ & $11.8 \pm 2.7$ & $1.25 \pm 0.18$ \\
\hline Anetium citrifolium & Pteridaceae (F) & $\mathrm{C} 3$ & 13 & $26.3 \pm 8.4$ & $0.14 \pm 0.03$ & $0.35 \pm 0.08$ & $250 \pm 68$ & $12.7 \pm 3.1$ & $51.0 \pm 15.1$ & 0.37 & 52.2 & 2 & $-32.2 \pm 0.4$ & $-0.9 \pm 0.2$ & $19.1 \pm 4.3$ & $0.77 \pm 0.09$ \\
\hline Anthurium acutangulum & Araceae & $\mathrm{C} 3$ & 13 & $16.2 \pm 3.6$ & $0.17 \pm 0.09$ & $0.41 \pm 0.05$ & $362 \pm 112$ & $7.7 \pm 1.9$ & $50.2 \pm 16.6$ & 0.39 & 50.2 & 2 & $-33.3 \pm 1.2$ & $-2.1 \pm 0.7$ & $10.9 \pm 1.1$ & $0.66 \pm 0.31$ \\
\hline Anthurium brownii & Araceae & $\mathrm{C} 3$ & 10 & $13.3 \pm 4.8$ & $0.29 \pm 0.04$ & $0.30 \pm 0.06$ & $141 \pm 98$ & $7.5 \pm 3.7$ & $56.7 \pm 20.7$ & 0.46 & 58.9 & 3 & $-27.3 \pm 1.2$ & $-4.4 \pm 1.9$ & $10.0 \pm 0.6$ & $1.08 \pm 0.22$ \\
\hline Anthurium clavigerum & Araceae & $\mathrm{C} 3$ & 11 & $35.5 \pm 15.0$ & $0.12 \pm 0.03$ & $0.20 \pm 0.05$ & $231 \pm 36$ & $12.5 \pm 4.6$ & $38.1 \pm 13.8$ & 0.41 & 55.2 & 3 & $-32.6 \pm 2.0$ & $-0.8 \pm 1.1$ & $25.3 \pm 14.6$ & $0.93 \pm 0.43$ \\
\hline Anthurium durandii & Araceae & $\mathrm{C} 3$ & 10 & $8.0 \pm 2.7$ & $0.22 \pm 0.03$ & $0.62 \pm 0.14$ & $490 \pm 97$ & $6.3 \pm 2.9$ & $90.9 \pm 38.5$ & 0.40 & 48.8 & 2 & $-25.8 \pm 0.9$ & $-3.7 \pm 0.8$ & $7.7 \pm 0.4$ & $1.40 \pm 0.67$ \\
\hline Anthurium friedrichssthalii & Araceae & $\mathrm{C} 3$ & 20 & $13.6 \pm 4.8$ & $0.17 \pm 0.04$ & $0.51 \pm 0.12$ & $402 \pm 96$ & $7.5 \pm 2.1$ & $59.7 \pm 21.2$ & 0.39 & 57.6 & 10 & $-30.4 \pm 2.5$ & $-2.8 \pm 2.4$ & $9.7 \pm 4.2$ & $0.84 \pm 0.32$ \\
\hline Anthurium hacumense & Araceae & $\mathrm{C} 3$ & 12 & $16.1 \pm 5.2$ & $0.12 \pm 0.03$ & $0.51 \pm 0.05$ & $366 \pm 237$ & $9.8 \pm 2.2$ & $63.1 \pm 12.8$ & 0.40 & 49.3 & 6 & $-30.4 \pm 2.4$ & $-2.0 \pm 1.3$ & $12.4 \pm 3.3$ & $0.77 \pm 0.28$ \\
\hline Anthurium scandens & Araceae & $\mathrm{C} 3$ & 12 & $9.7 \pm 1.7$ & $0.21 \pm 0.02$ & $0.48 \pm 0.07$ & $394 \pm 41$ & $7.1 \pm 1.6$ & $75.3 \pm 15.1$ & 0.28 & 35.6 & 3 & $-26.7 \pm 1.2$ & $-3.6 \pm 0.9$ & $8.5 \pm 1.3$ & $1.03 \pm 0.05$ \\
\hline Anthurium sp. & Araceae & $\mathrm{C} 3$ & 2 & $44.1 \pm 20.9$ & $0.10 \pm 0.02$ & $0.28 \pm 0.03$ & $235 \pm 72$ & $12.4 \pm 8.6$ & $26.5 \pm 7.0$ & 0.43 & 31.4 & 0 & - & - & & - \\
\hline Antrophyum lanceolatum & Pteridaceae (F) & $\mathrm{C} 3$ & 13 & $19.6 \pm 7.9$ & $0.51 \pm 0.21$ & $0.35 \pm 0.12$ & $97 \pm 97$ & $7.3 \pm 1.9$ & $40.5 \pm 14.2$ & 0.54 & 63.1 & 1 & $-32.1 \pm 0.0$ & $-0.4 \pm 0.0$ & $13.4 \pm 0.0$ & $0.61 \pm 0.00$ \\
\hline Aspasia principissa & Orchidaceae & $\mathrm{C} 3$ & 13 & $17.8 \pm 11.3$ & $0.18 \pm 0.03$ & $0.37 \pm 0.19$ & $386 \pm 253$ & $7.3 \pm 4.8$ & $42.4 \pm 7.6$ & 0.54 & 62.3 & 3 & $-31.0 \pm 2.9$ & $-2.1 \pm 0.4$ & $9.1 \pm 2.3$ & $0.61 \pm 0.27$ \\
\hline Asplenium juglandifolium & Aspleniaceae (F) & $\mathrm{C} 3$ & 10 & $22.2 \pm 6.2$ & $0.45 \pm 0.24$ & $0.24 \pm 0.11$ & $100 \pm 80$ & $6.9 \pm 1.0$ & $33.0 \pm 9.2$ & 0.50 & 59.0 & 2 & $-31.6 \pm 0.9$ & $-1.0 \pm 1.6$ & $13.8 \pm 1.6$ & $0.66 \pm 0.27$ \\
\hline Asplenium serratum & Aspleniaceae (F) & $\mathrm{C} 3$ & 10 & $25.9 \pm 12.1$ & $0.28 \pm 0.15$ & $0.19 \pm 0.08$ & $163 \pm 120$ & $9.7 \pm 4.9$ & $40.8 \pm 18.1$ & 0.58 & 68.6 & 2 & $-31.8 \pm 0.7$ & $-1.7 \pm 0.1$ & $15.0 \pm 8.5$ & $1.12 \pm 0.81$ \\
\hline Campylocentrum micranthum & Orchidaceae & CAM & 3 & $14.7 \pm 2.0$ & $0.12 \pm 0.01$ & $0.59 \pm 0.02$ & $485 \pm 4$ & $5.9 \pm 1.2$ & $40.3 \pm 7.8$ & 0.24 & 16.2 & 3 & $-14.0 \pm 1.1$ & $-1.1 \pm 1.9$ & $9.0 \pm 1.3$ & $0.61 \pm 0.04$ \\
\hline Campyloneurum aphanophlebium & Polypodiaceae (F) & C3 & 43 & $13.9 \pm 7.4$ & $0.49 \pm 0.16$ & $0.21 \pm 0.07$ & $108 \pm 68$ & $10.6 \pm 2.8$ & $85.9 \pm 28.7$ & 0.49 & 67.8 & 3 & $-29.3 \pm 2.2$ & $-1.4 \pm 2.2$ & $13.7 \pm 4.8$ & $1.39 \pm 0.36$ \\
\hline Campyloneurum phyllitidis & Polypodiaceae (F) & $\mathrm{C} 3$ & 13 & $13.7 \pm 4.0$ & $0.41 \pm 0.10$ & $0.20 \pm 0.07$ & $122 \pm 57$ & $9.0 \pm 3.4$ & $68.4 \pm 22.5$ & 0.43 & 56.1 & 3 & $-30.2 \pm 2.4$ & $-2.3 \pm 1.3$ & $13.8 \pm 9.3$ & $0.86 \pm 0.26$ \\
\hline Catasetum viridiflavum & Orchidaceae & $\mathrm{C} 3$ & 9 & $28.7 \pm 4.9$ & $0.16 \pm 0.02$ & $0.25 \pm 0.05$ & $185 \pm 38$ & $12.2 \pm 1.3$ & $43.5 \pm 6.9$ & 0.28 & 35.0 & 3 & $-29.7 \pm 1.7$ & $-0.5 \pm 1.5$ & $33.2 \pm 6.5$ & $1.27 \pm 0.28$ \\
\hline Catopsis sessiliflora & Bromeliaceae & $\mathrm{C} 3$ & 10 & $25.1 \pm 6.7$ & $0.14 \pm 0.03$ & $0.29 \pm 0.04$ & $275 \pm 62$ & $9.7 \pm 2.6$ & $40.5 \pm 13.1$ & 0.35 & 48.0 & 3 & $-29.0 \pm 2.5$ & $-3.2 \pm 0.8$ & $10.3 \pm 1.5$ & $0.40 \pm 0.03$ \\
\hline Caularthron bilamellatum & Orchidaceae & $\mathrm{C} 3$ & 1 & $8.9 \pm 0.0$ & $0.15 \pm 0.00$ & $0.94 \pm 0.00$ & $626 \pm 0$ & $3.3 \pm 0.0$ & $36.9 \pm 0.0$ & - & - & 0 & - & - & - & - \\
\hline Christensonella uncata & Orchidaceae & $\mathrm{C} 3$ & 96 & $7.7 \pm 1.5$ & $0.13 \pm 0.03$ & $1.34 \pm 0.29$ & $933 \pm 189$ & $2.8 \pm 0.8$ & $37.5 \pm 11.9$ & 0.35 & 59.9 & 4 & $-30.7 \pm 2.0$ & $-1.8 \pm 1.1$ & $9.3 \pm 0.8$ & $1.12 \pm 0.31$ \\
\hline Clusia cf. uvitana & Clusiaceae & $\mathrm{C} 3$ & 45 & $13.9 \pm 5.6$ & $0.20 \pm 0.06$ & $0.44 \pm 0.15$ & $346 \pm 103$ & $4.3 \pm 1.7$ & $33.5 \pm 14.4$ & 0.45 & 66.1 & 0 & - & - & - & - \\
\hline Codonanthe macradenia & Gesneriaceae & С3 & 26 & $15.6 \pm 7.7$ & $0.06 \pm 0.02$ & $1.75 \pm 0.59$ & $1080 \pm 286$ & $5.3 \pm 3.3$ & $33.2 \pm 14.7$ & 0.48 & 67.6 & 8 & $-27.2 \pm 4.6$ & $-3.3 \pm 1.7$ & $7.4 \pm 2.8$ & $0.58 \pm 0.22$ \\
\hline Columnea billbergiana & Gesneriaceae & $\mathrm{C} 3$ & 3 & $28.1 \pm 7.4$ & $0.05 \pm 0.01$ & $1.12 \pm 0.38$ & $691 \pm 205$ & $8.5 \pm 4.0$ & $29.5 \pm 7.7$ & 0.39 & 35.1 & 2 & $-30.8 \pm 1.2$ & $-2.7 \pm 0.6$ & $12.0 \pm 4.0$ & $0.48 \pm 0.05$ \\
\hline Cosmibuena grandiffora & Rubiaceae & $\mathrm{C} 3$ & 11 & $16.6 \pm 6.2$ & $0.10 \pm 0.02$ & $0.83 \pm 0.32$ & $682 \pm 254$ & $6.6 \pm 1.9$ & $43.3 \pm 14.2$ & 0.42 & 55.6 & 3 & $-31.2 \pm 0.8$ & $-4.5 \pm 2.3$ & $9.0 \pm 3.5$ & $0.82 \pm 0.17$ \\
\hline Dichaea panamensis & Orchidaceae & $\mathrm{C} 3$ & 19 & $25.2 \pm 12.3$ & $0.29 \pm 0.13$ & $0.18 \pm 0.07$ & $135 \pm 79$ & $8.8 \pm 5.2$ & $34.5 \pm 14.8$ & 0.56 & 71.4 & 0 & - & - & - & - \\
\hline Dicranoglossum panamense & Polypodiaceae (F) & $\mathrm{C} 3$ & 14 & $16.3 \pm 5.8$ & $0.45 \pm 0.19$ & $0.18 \pm 0.06$ & $101 \pm 64$ & $5.4 \pm 2.0$ & $32.8 \pm 5.2$ & 0.47 & 60.6 & 7 & $-30.0 \pm 1.5$ & $-3.0 \pm 0.3$ & $8.9 \pm 1.9$ & $0.66 \pm 0.20$ \\
\hline Dimerandra emarginata & Orchidaceae & $\mathrm{C} 3$ & 2 & $9.3 \pm 0.6$ & $0.25 \pm 0.02$ & $0.41 \pm 0.04$ & $319 \pm 52$ & $4.2 \pm 0.8$ & $44.6 \pm 5.9$ & 0.25 & 13.5 & 0 & - & - & - & - \\
\hline Elaphoglossum doanense & Dryopteridaceae (F) & $\mathrm{C} 3$ & 11 & $11.5 \pm 2.5$ & $0.29 \pm 0.10$ & $0.30 \pm 0.06$ & $251 \pm 75$ & $5.3 \pm 1.7$ & $52.1 \pm 10.0$ & 0.37 & 51.7 & 3 & $-31.5 \pm 2.3$ & $-2.8 \pm 0.5$ & $9.2 \pm 1.7$ & $0.88 \pm 0.09$ \\
\hline Elaphoglossum herminieri & Dryopteridaceae (F) & $\mathrm{C} 3$ & 79 & $7.6 \pm 2.6$ & $0.27 \pm 0.05$ & $0.45 \pm 0.10$ & $412 \pm 227$ & $4.6 \pm 1.6$ & $62.7 \pm 19.1$ & 0.42 & 66.5 & 9 & $-31.3 \pm 1.7$ & $-2.1 \pm 1.2$ & $9.0 \pm 2.0$ & $1.89 \pm 1.51$ \\
\hline Elaphoglossum sporadolepis & Dryopteridaceae (F) & $\mathrm{C} 3$ & 53 & $8.0 \pm 3.0$ & $0.30 \pm 0.07$ & $0.34 \pm 0.07$ & $344 \pm 153$ & $4.2 \pm 1.8$ & $51.5 \pm 15.7$ & 0.43 & 68.6 & 9 & $-30.2 \pm 1.4$ & $-3.6 \pm 1.4$ & $6.8 \pm 1.9$ & $0.87 \pm 0.15$ \\
\hline Elleanthus longibracteatus & Orchidaceae & $\mathrm{C} 3$ & 5 & $9.1 \pm 1.4$ & $0.38 \pm 0.03$ & $0.23 \pm 0.02$ & $184 \pm 17$ & $4.9 \pm 1.1$ & $53.4 \pm 7.3$ & 0.26 & 22.8 & 3 & $-29.9 \pm 1.3$ & $-3.1 \pm 1.0$ & $8.4 \pm 0.6$ & $0.96 \pm 0.14$ \\
\hline Epidendrum difforme & Orchidaceae & CAM & 12 & $9.1 \pm 2.3$ & $0.07 \pm 0.01$ & $2.17 \pm 0.58$ & $1496 \pm 323$ & $2.5 \pm 0.9$ & $27.7 \pm 8.7$ & 0.36 & 47.4 & 3 & $-15.5 \pm 1.0$ & $-3.4 \pm 1.4$ & $7.1 \pm 1.5$ & $0.81 \pm 0.09$ \\
\hline Epidendrum nocturnum & Orchidaceae & $\mathrm{C} 3$ & 11 & $12.7 \pm 5.2$ & $0.14 \pm 0.03$ & $0.77 \pm 0.25$ & $532 \pm 144$ & $5.4 \pm 1.6$ & $48.0 \pm 19.3$ & 0.41 & 53.2 & 3 & $-23.9 \pm 2.0$ & $-3.0 \pm 0.4$ & $8.8 \pm 0.7$ & $0.89 \pm 0.50$ \\
\hline Epidendrum sp. & Orchidaceae & $\mathrm{C} 3$ & 3 & $17.0 \pm 12.4$ & $0.20 \pm 0.13$ & $0.69 \pm 0.49$ & $381 \pm 272$ & $5.5 \pm 0.9$ & $41.0 \pm 18.2$ & 0.63 & 54.2 & 0 & - & - & & - \\
\hline Gongora quinquenervis & Orchidaceae & $\mathrm{C} 3$ & 3 & $25.9 \pm 10.1$ & $0.14 \pm 0.05$ & $0.26 \pm 0.02$ & $268 \pm 38$ & $7.9 \pm 3.1$ & $30.4 \pm 0.6$ & 0.34 & 28.8 & 2 & $-32.5 \pm 3.1$ & $-4.0 \pm 1.3$ & $10.6 \pm 2.3$ & $0.52 \pm 0.06$ \\
\hline Guzmania subcorymbosa & Bromeliaceae & $\mathrm{C} 3$ & 13 & $21.1 \pm 4.2$ & $0.24 \pm 0.03$ & $0.18 \pm 0.02$ & $155 \pm 17$ & $3.6 \pm 0.8$ & $17.5 \pm 5.1$ & 0.30 & 37.7 & 2 & $-30.6 \pm 0.5$ & $-2.0 \pm 1.7$ & $4.8 \pm 0.1$ & $0.24 \pm 0.06$ \\
\hline Heterotaxis discolor & Orchidaceae & $\mathrm{C}_{3}$ & 1 & $4.4 \pm 0.0$ & $0.17 \pm 0.00$ & $1.44 \pm 0.00$ & $1130 \pm 0$ & $1.6 \pm 0.0$ & $35.9 \pm 0.0$ & & & & & & & \\
\hline
\end{tabular}




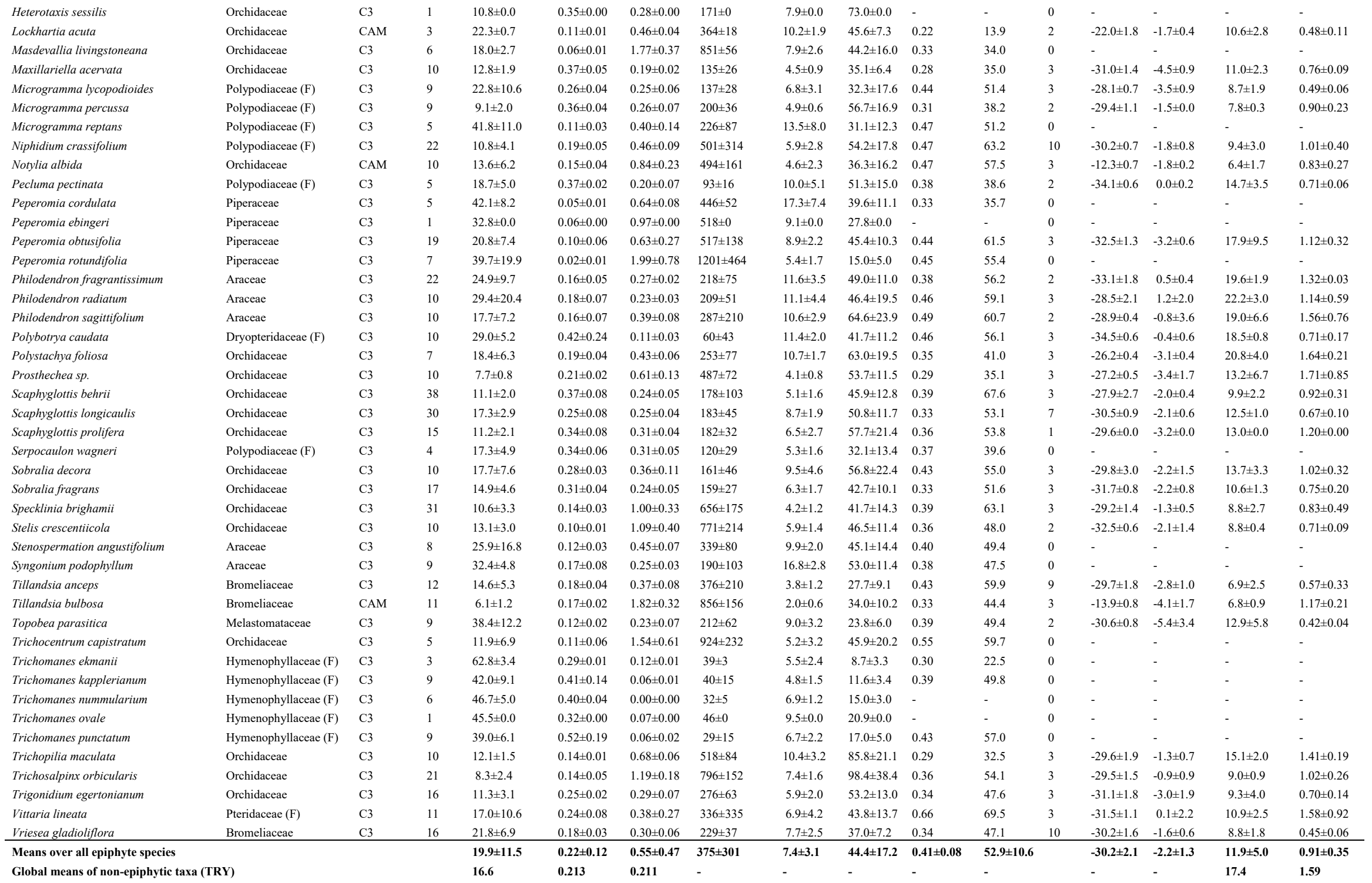


Table A.2. AIC-based comparisons of generalized linear mixed models (GLMMs) analyzing the relationship between leaf traits (dependent variable; SLA: specific leaf area, LDMC: leaf dry matter content, Thickness: leaf thickness, $\mathrm{LWC}_{\text {area: }}$ leaf water content per leaf area, $\mathrm{Chl}_{\text {mass }}$ : mass-based chlorophyll concentration, Chl area: area-based chlorophyll concentration, $\mathrm{N}_{\text {mass }}$ : mass-based nitrogen concentration, $\mathrm{N}_{\text {area }}$ : area-based nitrogen concentration, $\delta^{13} \mathrm{C}$ : carbon isotope ratio, $\delta^{15} \mathrm{~N}$ : nitrogen isotope ratio) and different fixed effects (height, height ${ }^{2}$, taxonomic group). Only epiphytes belonging to the four major taxonomic groups (aroids, bromeliads, orchids, ferns) were considered. At first, GLMMs with full fixed effects (mode19) and all possible combinations of random effects (no random effect, random intercept, random intercept and variance, random intercept and slope, random intercept and slope and variance by species) were fitted to trait data (n: number of sampled individuals) to obtain the minimal adequate random structure using the REML estimation method (Zuur et al. 2009). The minimal adequate random structure for each trait is given as Random effect (I: random intercept; S: random slope; V: random variance). In a second step, using the minimal adequate random structure for each trait, we compared GLMMs with all meaningful fixed-effect combinations (see annotation below table) using the ML estimation method to obtain the minimal adequate model (MAM) based on AIC values $(\triangle \mathrm{AIC}=\mathrm{AIC}$ focal model $-\mathrm{AIC}$ MAM $)$. Simpler models were preferred to more complex models when $\triangle \mathrm{AIC} \leq 10$ (Burnham \& Anderson 2004). Note that the nine models are sorted by model complexity, with model9 being the most complex. The MAM is marked by grey color.

\begin{tabular}{|c|c|c|c|c|c|c|c|c|c|c|c|}
\hline Leaf trait & $\mathbf{n}$ & $\begin{array}{c}\text { Random } \\
\text { effect }\end{array}$ & $\begin{array}{l}\triangle \mathrm{AIC} \\
\text { mod1 }^{*}\end{array}$ & $\begin{array}{l}\triangle \mathrm{AIC} \\
\operatorname{mod2}{ }^{*}\end{array}$ & $\begin{array}{l}\triangle \mathrm{AIC} \\
\operatorname{mod3}^{*}\end{array}$ & $\begin{array}{l}\triangle \mathrm{AIC} \\
\operatorname{mod4}^{*}\end{array}$ & $\begin{array}{l}\triangle \mathrm{AIC} \\
\operatorname{mod5}^{*}\end{array}$ & $\begin{array}{l}\triangle \mathrm{AIC} \\
\operatorname{mod6}^{*}\end{array}$ & $\begin{array}{l}\triangle \mathrm{AIC} \\
\bmod 7^{*}\end{array}$ & $\begin{array}{l}\triangle \mathrm{AIC} \\
\operatorname{mod8}^{*}\end{array}$ & $\begin{array}{l}\triangle \mathrm{AIC} \\
\bmod 9^{*}\end{array}$ \\
\hline SLA & 1022 & $\mathrm{I}+\mathrm{S}+\mathrm{V}$ & 30.6 & 77.6 & 36.6 & 33.3 & 0.0 & 4.9 & 7.9 & 7.7 & 6.7 \\
\hline LDMC & 1021 & $\mathrm{I}+\mathrm{S}+\mathrm{V}$ & 34.7 & 17.6 & 0.0 & 4.9 & 36.2 & 0.8 & 5.8 & 5.9 & 11.5 \\
\hline $\mathbf{L W C}_{\text {area }}$ & 1016 & $\mathrm{I}+\mathrm{S}+\mathrm{V}$ & 14.5 & 3.8 & 0.0 & 4.0 & 15.5 & 1.7 & 5.6 & 6.7 & 9.9 \\
\hline Chl mass & 1023 & $\mathrm{I}+\mathrm{S}+\mathrm{V}$ & 10.9 & 31.2 & 3.6 & 5.1 & 6.0 & 0.0 & 2.2 & 3.1 & 7.9 \\
\hline $\mathrm{Chl}_{\text {area }}$ & 1025 & $\mathrm{I}+\mathrm{S}+\mathrm{V}$ & 16.4 & 27.4 & 12.5 & 81.1 & 11.6 & 5.9 & 4.3 & 6.0 & 0.0 \\
\hline$\delta^{13} \mathrm{C}$ & 184 & I & 3.7 & 67.8 & 4.0 & 6.5 & 1.0 & 0.0 & 4.6 & 5.1 & 9.1 \\
\hline$\delta^{15} \mathbf{N}$ & 202 & $\mathrm{I}+\mathrm{V}$ & 0.0 & 7.9 & 3.5 & 8.5 & 0.9 & 4.4 & 9.5 & 9.9 & 8.2 \\
\hline
\end{tabular}

*Fixed effect structures:

model1: Trait $\sim$ Height

model2: Trait $\sim$ TaxGroup

model3: Trait $\sim$ Height + TaxGroup

model4: Trait $\sim$ Height $x$ TaxGroup

model5: Trait $\sim$ Height + Height $^{2}$

model6: Trait $\sim$ Height + Height $^{2}+$ TaxGroup

model7: Trait $\sim$ Height $x$ TaxGroup + Height $^{2}$

model8: Trait $\sim$ Height + Height $^{2} \mathrm{x}$ TaxGroup

mode19: Trait $\sim$ Height $\mathrm{x}$ TaxGroup + Height $^{2} \mathrm{x}$ TaxGroup 
Table A.3. Summary statistics of linear models (LMs) testing trait-height relationships of vascular epiphytes for 10 leaf traits (SLA: specific leaf area, LDMC: leaf dry matter content, Thickness: leaf thickness, LWC $_{\text {area: }}$ leaf water content per leaf area, $\mathrm{Ch}_{\text {mass }}$ : mass-based chlorophyll concentration, $\mathrm{Ch}_{\text {area }}$ : area-based chlorophyll concentration, $\mathrm{N}_{\text {mass }}$ : mass-based nitrogen concentration, $\mathrm{N}_{\text {area }}$ : area-based nitrogen concentration, $\delta^{13} \mathrm{C}$ : carbon isotope ratio, $\delta^{15} \mathrm{~N}$ : nitrogen isotope ratio). Trait-height relationships were analyzed by comparing simple LMs (trait $\sim$ height) and LMs including a quadratic term (trait $\sim$ height \pm height ${ }^{2}$ ) based on AIC values. We selected the non-linear LM as minimum adequate model (MAM) if it was superior by $\triangle \mathrm{AIC}>10$ (Burnham \& Anderson 2004). For each trait, LMs were applied to the entire dataset consisting of all sampled individuals, as well as to the community mean trait values calculated for all 1-m height intervals. Int, a, b: model coefficients of the MAM (trait $\sim \operatorname{Int} \pm \mathrm{a}^{*}$ height $\pm \mathrm{b}^{*}$ height ${ }^{2}$ ). $P$ values are given separately for the linear term and the quadratic term.

\begin{tabular}{|c|c|c|c|c|c|c|c|c|c|}
\hline Leaf trait & Data set & МАM & df & $\mathbf{R}^{2}$ & Int & $\mathbf{a}$ & $\mathbf{b}$ & $P$ (linear) & $P$ (quadratic) \\
\hline SLA & Community means & Non-linear & 30 & 0.89 & 35.19 & -2.11 & 0.05 & $<0.001$ & $<0.001$ \\
\hline SLA & All individuals & Non-linear & 1145 & 0.30 & 34.06 & -2.02 & 0.04 & $<0.001$ & $<0.001$ \\
\hline LDMC & Community means & Linear & 31 & 0.30 & 0.28 & 0.00 & - & 0.001 & - \\
\hline LDMC & All individuals & Linear & 1144 & 0.01 & 0.26 & 0.00 & - & $<0.001$ & - \\
\hline Thickness & Community means & Linear & 31 & 0.72 & 0.24 & 0.02 & - & $<0.001$ & - \\
\hline Thickness & All individuals & Linear & 1142 & 0.11 & 0.22 & 0.02 & - & $<0.001$ & - \\
\hline $\mathbf{L W C}_{\text {area }}$ & Community means & Linear & 31 & 0.64 & 189.33 & 11.76 & - & $<0.001$ & - \\
\hline $\mathbf{L W C}_{\text {area }}$ & All individuals & Linear & 1139 & 0.11 & 185.38 & 13.76 & - & $<0.001$ & - \\
\hline $\mathrm{Chl}_{\text {mass }}$ & Community means & Non-linear & 30 & 0.76 & 11.98 & -0.55 & 0.01 & $<0.001$ & $<0.001$ \\
\hline $\mathrm{Chl}_{\text {mass }}$ & All individuals & Non-linear & 1146 & 0.16 & 11.00 & -0.44 & 0.01 & $<0.001$ & $<0.001$ \\
\hline Chl $_{\text {area }}$ & Community means & Linear & 31 & 0.26 & 42.16 & 0.32 & - & 0.002 & - \\
\hline $\mathrm{Chl}_{\text {area }}$ & All individuals & Non-linear & 1148 & 0.02 & 36.36 & 1.45 & -0.04 & $<0.001$ & $<0.001$ \\
\hline $\mathbf{N}_{\text {mass }}$ & Community means & Non-linear & 30 & 0.39 & 19.09 & -0.95 & 0.02 & $<0.001$ & 0.001 \\
\hline $\mathbf{N}_{\text {mass }}$ & All individuals & Non-linear & 217 & 0.09 & 16.79 & -0.70 & 0.02 & $<0.001$ & $<0.001$ \\
\hline $\mathbf{N}_{\text {area }}$ & Community means & Linear & 31 & 0.13 & 0.79 & 0.01 & - & 0.042 & - \\
\hline $\mathbf{N}_{\text {area }}$ & All individuals & Linear & 216 & 0.04 & 0.71 & 0.01 & - & 0.005 & - \\
\hline$\delta^{13} \mathrm{C}$ & Community means & Linear & 31 & 0.66 & -33.46 & 0.19 & - & $<0.001$ & - \\
\hline$\delta^{13} \mathrm{C}$ & All individuals & Linear & 200 & 0.35 & -32.93 & 0.18 & - & $<0.001$ & - \\
\hline$\delta^{15} \mathbf{N}$ & Community means & Linear & 31 & 0.23 & -1.42 & -0.04 & - & 0.004 & - \\
\hline$\delta^{15} \mathbf{N}$ & All individuals & Linear & 218 & 0.05 & -1.59 & -0.05 & - & $<0.001$ & - \\
\hline
\end{tabular}


Table A.4. Summary statistics of linear models (LMs) testing trait-height relationships of vascular epiphytes for 6 leaf traits (SLA: specific leaf area, LDMC: leaf dry matter content, Thickness: leaf thickness, LWC $_{\text {area: }}$ leaf water content per leaf area, Chl mass: mass-based chlorophyll concentration, $\mathrm{Chl}_{\text {area }}$ : area-based chlorophyll concentration). Traitheight relationships were analyzed by comparing simple LMs (trait $\sim$ height) and LMs including a quadratic term (trait $\sim$ height \pm height ${ }^{2}$ ) based on AIC values. We selected the non-linear LM as minimum adequate model (MAM) if it was superior by $\triangle \mathrm{AIC}>10$ (Burnham \& Anderson 2004). In contrast to Table A.3, the LMs were applied to the dataset consisting of the predicted trait values for all individuals recorded in the comprehensive census (see Fig. S2 for details), as well as to the community trait means based on these. The large numbers of degrees of freedom (df) result from the repetition of the prediction procedure for 100 times (Fig. A.2). Int, a, b: model coefficients of the MAM (trait $\sim$ Int \pm $\mathrm{a}^{*}$ height $\pm \mathrm{b}^{*}$ height $\left.{ }^{2}\right) . P$ values are given separately for the linear term and the quadratic term.

\begin{tabular}{|c|c|c|c|c|c|c|c|c|c|}
\hline Leaf trait & Data set & MAM & df & $\mathbf{R}^{2}$ & Int & a & b & $P$ (linear) & $P$ (quadratic) \\
\hline SLA & Community means & Non-linear & 3297 & 0.96 & 31.26 & -1.54 & 0.03 & $<0.001$ & $<0.001$ \\
\hline SLA & All individuals & Non-linear & 2034797 & 0.28 & 31.58 & -1.70 & 0.04 & $<0.001$ & $<0.001$ \\
\hline LDMC & Community means & Non-linear & 3297 & 0.38 & 0.22 & 0.01 & 0.00 & $<0.001$ & $<0.001$ \\
\hline LDMC & All individuals & Non-linear & 2034797 & 0.01 & 0.22 & 0.01 & 0.00 & $<0.001$ & $<0.001$ \\
\hline Thickness & Community means & Non-linear & 3297 & 0.76 & 0.35 & 0.01 & 0.00 & $<0.001$ & $<0.001$ \\
\hline Thickness & All individuals & Non-linear & 2028497 & 0.04 & 0.32 & 0.01 & 0.00 & $<0.001$ & $<0.001$ \\
\hline $\mathbf{L W C}_{\text {area }}$ & Community means & Non-linear & 3297 & 0.76 & 286.07 & 0.79 & 0.29 & 0.074 & $<0.001$ \\
\hline $\mathbf{L W C}_{\text {area }}$ & All individuals & Non-linear & 2034797 & 0.04 & 260.90 & 6.09 & 0.08 & $<0.001$ & $<0.001$ \\
\hline $\mathrm{Chl}_{\text {mass }}$ & Community means & Non-linear & 3297 & 0.92 & 11.23 & -0.40 & 0.01 & $<0.001$ & $<0.001$ \\
\hline $\mathrm{Chl}_{\text {mass }}$ & All individuals & Non-linear & 2034797 & 0.13 & 11.15 & -0.41 & 0.01 & $<0.001$ & $<0.001$ \\
\hline $\mathrm{Chl}_{\text {area }}$ & Community means & Non-linear & 3297 & 0.72 & 38.33 & 1.25 & -0.03 & $<0.001$ & $<0.001$ \\
\hline Chl $_{\text {area }}$ & All individuals & Non-linear & 2034797 & 0.04 & 37.42 & 1.57 & -0.04 & $<0.001$ & $<0.001$ \\
\hline
\end{tabular}

Table A.5. Pairwise correlations between leaf traits of vascular epiphytes. Pearson's correlation coefficient is reported for all significant correlations $(P<0.05)$. Bold numbers indicate strong correlations $(r>0.5)$.

\begin{tabular}{|c|c|c|c|c|c|c|c|c|c|c|}
\hline & SLA & Chl ${ }_{\text {area }}$ & Chl $_{\text {mass }}$ & $\mathbf{L W C}_{\text {area }}$ & Thickness & LDMC & $\delta^{15} \mathrm{~N}$ & $\delta^{13} \mathrm{C}$ & $\mathbf{N}_{\text {area }}$ & $\mathbf{N}_{\text {mass }}$ \\
\hline SLA & & -0.42 & 0.64 & -0.51 & -0.48 & -0.16 & 0.22 & -0.24 & -0.62 & 0.46 \\
\hline $\mathrm{Chl}_{\text {area }}$ & -0.42 & & 0.42 & 0.09 & - & 0.21 & 0.10 & -0.13 & 0.56 & 0.36 \\
\hline $\mathrm{Chl}_{\text {mass }}$ & 0.64 & 0.42 & & -0.44 & -0.41 & - & 0.30 & -0.31 & -0.12 & 0.67 \\
\hline $\mathbf{L W C}_{\text {area }}$ & -0.51 & 0.09 & -0.44 & & 0.84 & -0.72 & -0.21 & 0.37 & 0.19 & -0.35 \\
\hline Thickness & -0.48 & - & -0.41 & 0.84 & & -0.66 & -0.15 & 0.48 & 0.13 & -0.33 \\
\hline LDMC & -0.16 & 0.21 & - & -0.72 & -0.66 & & - & -0.28 & 0.17 & 0.41 \\
\hline$\delta^{15} \mathbf{N}$ & 0.22 & 0.10 & 0.30 & -0.21 & -0.15 & - & & -0.15 & 0.13 & 0.41 \\
\hline$\delta^{13} \mathrm{C}$ & -0.24 & -0.13 & -0.31 & 0.37 & 0.48 & -0.28 & -0.15 & & - & -0.26 \\
\hline $\mathbf{N}_{\text {area }}$ & -0.62 & 0.56 & -0.12 & 0.19 & 0.13 & 0.17 & 0.13 & - & & 0.14 \\
\hline $\mathbf{N}_{\text {mass }}$ & 0.46 & 0.36 & 0.67 & -0.35 & -0.33 & 0.41 & 0.41 & -0.26 & 0.14 & \\
\hline
\end{tabular}


Table A.6. AIC-based comparisons of linear models (LMs) analyzing the relationship between leaf traits (dependent variable; SLA: specific leaf area, LDMC: leaf dry matter content, Thickness: leaf thickness, $\mathrm{LWC}_{\text {area }}$ : leaf water content per leaf area, Chl mass: mass-based chlorophyll concentration, Chlarea: area-based chlorophyll concentration) and different fixed effects (height, species). The LMs with all meaningful fixed effect combinations are given as annotation below the table; note that these models are sorted by their complexity. To obtain the minimal adequate model (MAM), we compared the different LMs based on their AIC values $\left(\triangle \mathrm{AIC}=\mathrm{AIC}_{\text {focal }}\right.$ model $-\mathrm{AIC}$ MAM $)$. Simpler LMs were preferred to more complex models when $\triangle \mathrm{AIC} \leq 10$ (Burnham \& Anderson 2004). The MAM is marked by grey color. Because we used species identity as fixed effect, we only considered species with $\geq 10$ records per trait.

\begin{tabular}{|c|c|c|c|c|c|c|}
\hline Leaf trait & $\mathbf{n}$ & $\begin{array}{c}\triangle \mathrm{AIC} \\
\text { model1 }^{*}\end{array}$ & $\begin{array}{c}\triangle \mathrm{AIC} \\
\text { model2* }\end{array}$ & $\begin{array}{c}\triangle \mathrm{AIC} \\
\text { model3 }^{*}\end{array}$ & $\begin{array}{c}\Delta \mathrm{AIC} \\
\text { model4 }^{*}\end{array}$ & $\begin{array}{c}\triangle \mathrm{AIC} \\
\text { model5 }^{*}\end{array}$ \\
\hline SLA & 988 & 988.0 & 737.7 & 327.6 & 184.3 & 0.0 \\
\hline LDMC & 986 & 1081.8 & 1077.6 & 87.7 & 78.5 & 0.0 \\
\hline Thickness & 991 & 1871.3 & 1720.2 & 67.1 & 40.8 & 0.0 \\
\hline $\mathbf{L W C}_{\text {area }}$ & 981 & 1422.5 & 1293.6 & 8.3 & 0.0 & 7.6 \\
\hline Chl mass & 989 & 850.6 & 649.9 & 113.2 & 46.5 & 0.0 \\
\hline Chl $_{\text {area }}$ & 991 & 593.8 & 594.1 & 18.8 & 0.0 & 37.7 \\
\hline
\end{tabular}

Table A.7. Proportion of species with significant trait-height relationships for six leaf traits (SLA: specific leaf area, LDMC: leaf dry matter content, Thickness: leaf thickness, $\mathrm{LWC}_{\text {area }}$ : leaf water content per leaf area, Chl mass: massbased chlorophyll concentration, $\mathrm{Chl}_{\text {area }}$ : area-based chlorophyll concentration). For each trait, the number of species with significant trait-height relationships $(P<0.05)$ was divided by the total number of species $(\mathrm{n}=51)$. Furthermore, significant trait-height relationships were subdivided into positive and negative relationships. See Figs. A.4-A.9 for more details on intraspecific trait-height relationships.

\begin{tabular}{lccc}
\hline Leaf trait & $\begin{array}{c}\text { \% significant } \\
\text { slopes }\end{array}$ & $\begin{array}{c}\text { \% significant slopes } \\
\text { (positive) }\end{array}$ & $\begin{array}{c}\text { \% significant slopes } \\
\text { (negative) }\end{array}$ \\
\hline SLA & 45.1 & 0.0 & 45.1 \\
LDMC & 33.3 & 27.5 & 5.9 \\
Thickness & 25.5 & 21.6 & 3.9 \\
LWC $_{\text {area }}$ & 15.7 & 11.8 & 3.9 \\
Chl $_{\text {mass }}$ & 19.6 & 0.0 & 19.6 \\
Chl $_{\text {area }}$ & 17.6 & 17.6 & 0.0 \\
\hline
\end{tabular}




\section{B Supplementary information to chapter 3}

Table B.1. List of vascular holoepiphytes found on the forest floor per study site. We did not surveyed ferns and aroids at the Brazilian sites. Species names follow the The Plant List (http://www.theplantlist.org/). Species found attached to branches are in bold. Vouchers of Brazilian species were deposited in the herbarium of the Federal University of Pernambuco and in the herbarium of the Federal University of Paraiba. Vouchers of the Panamanian species were deposited in the herbarium of the Smithsonian Tropical Research Institute, Panama.

\begin{tabular}{|c|c|c|c|c|c|}
\hline Brazil core & & Brazil edge & & Panama & \\
\hline Family & Species & Family & Species & Family & Species \\
\hline Bromeliaceae & Aechmea cf. stelligera & Bromeliaceae & $\begin{array}{l}\text { Aechmea cf. } \\
\text { stelligera }\end{array}$ & Araceae & Anthurium acutangulum \\
\hline Bromeliaceae & Tillandsia bulbosa & Bromeliaceae & Tillandsia bulbosa & Araceae & $\begin{array}{l}\text { Anthurium } \\
\text { friedrichsthallii }\end{array}$ \\
\hline Bromeliaceae & Tillandsia juncea & Bromeliaceae & Tillandsia juncea & Araceae & Anthurium hacumense \\
\hline Bromeliaceae & Tillandsia tenuifolia & Bromeliaceae & Tillandsia tenuifolia & Araceae & Anthurium scandens \\
\hline Bromeliaceae & Tillandsia usneoides & Bromeliaceae & Tillandsia usneoides & Araceae & $\begin{array}{l}\text { Stenospermation } \\
\text { angustifolium }\end{array}$ \\
\hline Cactaceae & Rhypsalis baccifera & Orchidaceae & $\begin{array}{l}\text { Campylocentrum } \\
\text { crassyrhyzum }\end{array}$ & Aspleniaceae & Asplenium serratum \\
\hline Orchidaceae & Anathallis sclerophylla & Orchidaceae & Cattleya labiata & Bromeliaceae & Catopsis sessiliflora \\
\hline Orchidaceae & $\begin{array}{l}\text { Campylocentrum } \\
\text { crassyrhyzum }\end{array}$ & Orchidaceae & $\begin{array}{l}\text { Dichaea } \\
\text { panamensis }\end{array}$ & Bromeliaceae & Guzmania subcorymbosa \\
\hline Orchidaceae & Cattleya labiata & Orchidaceae & $\begin{array}{l}\text { Dimerandra } \\
\text { emarginata }\end{array}$ & Bromeliaceae & Tillandsia anceps \\
\hline Orchidaceae & Dichaea panamensis & Orchidaceae & $\begin{array}{l}\text { Epidendrum } \\
\text { difforme }\end{array}$ & Bromeliaceae & Tillandsia bulbosa \\
\hline Orchidaceae & $\begin{array}{l}\text { Dimerandra } \\
\text { emarginata }\end{array}$ & Orchidaceae & $\begin{array}{l}\text { Epidendrum } \\
\text { nocturnum }\end{array}$ & Cactaceae & Epiphyllum phyllanthus \\
\hline Orchidaceae & Epidendrum difforme & Orchidaceae & Gomesa barbata & Gesneriaceae & Codonanthe macradenia \\
\hline Orchidaceae & Epidendrum riggidum & Orchidaceae & $\begin{array}{l}\text { Polystachya } \\
\text { concreta }\end{array}$ & Orchidaceae & $\begin{array}{l}\text { Campylocentrum } \\
\text { micranthum }\end{array}$ \\
\hline Orchidaceae & Gomesa barbata & Orchidaceae & $\begin{array}{l}\text { Rodrighezia } \\
\text { bahiensis }\end{array}$ & Orchidaceae & Catasetum viridiflavum \\
\hline Orchidaceae & Maxillaria ochroleuca & Orchidaceae & $\begin{array}{l}\text { Scaphyglottis } \\
\text { fusiformis }\end{array}$ & Orchidaceae & Christensonella uncata \\
\hline Orchidaceae & Notylia lyrata & Orchidaceae & Scaphyglottis sickii & Orchidaceae & Dichaea panamensis \\
\hline Orchidaceae & Polystachya concreta & & & Orchidaceae & Epidendrum difforme \\
\hline Orchidaceae & $\begin{array}{l}\text { Prosthechea } \\
\text { alagoensis }\end{array}$ & & & Orchidaceae & Epidendrum nocturnum \\
\hline Orchidaceae & Prosthechea fragrans & & & Orchidaceae & Heterotaxis sessilis \\
\hline Orchidaceae & Rodrighezia bahiensis & & & Orchidaceae & $\begin{array}{l}\text { Masdevallia } \\
\text { livingstoneana }\end{array}$ \\
\hline Orchidaceae & $\begin{array}{l}\text { Scaphyglottis } \\
\text { fusiformis }\end{array}$ & & & Orchidaceae & Mormodes powellii \\
\hline Orchidaceae & Scaphyglottis sickii & & & Orchidaceae & Polystachya foliosa \\
\hline \multirow[t]{16}{*}{ Orchidaceae } & $\begin{array}{l}\text { Trigonidium } \\
\text { acuminatum }\end{array}$ & & & Orchidaceae & Prosthechea aemula \\
\hline & & & & Orchidaceae & Scaphyglottis behrii \\
\hline & & & & Orchidaceae & Scaphyglottis longicaulis \\
\hline & & & & Orchidaceae & Sobralia fenzliana \\
\hline & & & & Orchidaceae & Sobralia fragans \\
\hline & & & & Orchidaceae & $\begin{array}{l}\text { Trichocentrum } \\
\text { capistratum }\end{array}$ \\
\hline & & & & Orchidaceae & Trichopilia maculata \\
\hline & & & & Orchidaceae & Trichosalpinx orbicularis \\
\hline & & & & Orchidaceae & $\begin{array}{l}\text { Trigonidium } \\
\text { egertonianum }\end{array}$ \\
\hline & & & & Piperaceae & $\begin{array}{l}\text { Peperomia cordulata } \\
\text { Peperomia rotundifolia }\end{array}$ \\
\hline & & & & Polypodiaceae & $\begin{array}{l}\text { Dicranoglossum } \\
\text { panamense }\end{array}$ \\
\hline & & & & Polypodiaceae & $\begin{array}{l}\text { Microgramma } \\
\text { lycopodioides }\end{array}$ \\
\hline & & & & Polypodiaceae & Microgramma percussa \\
\hline & & & & Polypodiaceae & Niphidium crassifolium \\
\hline & & & & Vittariaceae & $\begin{array}{l}\text { Ananthacorus } \\
\text { angustifolius }\end{array}$ \\
\hline & & & & Vittariaceae & Vittaria lineata \\
\hline Total & 23 & & 16 & & 27 \\
\hline
\end{tabular}




\section{Appendix}

Table B.2. List of vascular holoepiphytes found in the canopy per forest. Species names follow the Plant List (http://www.theplantlist.org/). Species found in the canopy above Brazilian core and Panamanian transects are indicated in bold, whereas species found in the Brazilian edge transects are indicated with asterisks. For Panamanian transect canopies, species occurring on substrate $<10 \mathrm{~cm}$ in diameter are indicated with $\mathrm{a}^{\dagger}$ symbol. Transect canopy information was based on own observations in the Brazilian sites (see main text) and on inventoried data in the Panamanian site (Glenda Mendieta-Leiva \& Gerhard Zotz, unpublished data). Vouchers of Brazilian species were deposited in the herbarium of the Federal University of Pernambuco and in the herbarium of the Federal University of Paraiba. Vouchers of the Panamanian species were deposited in the herbarium of the Smithsonian Tropical Research Institute, Panama.

\begin{tabular}{|c|c|c|c|}
\hline \multicolumn{2}{|l|}{ Brazil } & \multicolumn{2}{|l|}{ Panama } \\
\hline Family & Species & Family & Species \\
\hline Bromeliaceae & Aechmea stelligera* & Araceae & Anthurium acutangulum ${ }^{\dagger}$ \\
\hline Bromeliaceae & Aechmea fulgens & Araceae & Anthurium bakeri \\
\hline Bromeliaceae & Bilbergia morelii & Araceae & Anthurium brownii ${ }^{\dagger}$ \\
\hline Bromeliaceae & Canistrum alagoanum & Araceae & Anthurium clavigerum ${ }^{\dagger}$ \\
\hline Bromeliaceae & Guzmania lingulata & Araceae & Anthurium durandii ${ }^{\dagger}$ \\
\hline Bromeliaceae & Lymania smithii & Araceae & Anthurium friedrichsthalii ${ }^{\dagger}$ \\
\hline Bromeliaceae & Tillandsia bulbosa & Araceae & Anthurium hacumense $e^{\dagger}$ \\
\hline Bromeliaceae & Tillandsia juncea* & Araceae & Anthurium scandens ${ }^{\dagger}$ \\
\hline Bromeliaceae & Tillandsia stricta & Araceae & Philodendron radiatum ${ }^{\dagger}$ \\
\hline Bromeliaceae & Tillandsia tenuifolia * & Araceae & Philodendron sagittifolium ${ }^{\dagger}$ \\
\hline Bromeliaceae & Tillandsia usneoides* & Araceae & Stenospermation angustifolium \\
\hline Cactaceae & Rhypsalis baccifera & Aspleniaceae & Asplenium juglandifolium \\
\hline Cactaceae & Epiphyllum phyllanthus & Aspleniaceae & Asplenium serratum ${ }^{\dagger}$ \\
\hline Orchidaceae & Acianthera pernambucensis & Bromeliaceae & Aechmea tillandsioides ${ }^{\dagger}$ \\
\hline Orchidaceae & Anathallis brevipes & Bromeliaceae & Catopsis sessiliflora $^{\dagger}$ \\
\hline Orchidaceae & Anathallis sclerophylla & Bromeliaceae & Guzmania musaica \\
\hline Orchidaceae & Campylocentrum amazonicum & Bromeliaceae & Guzmania subcorymbosa ${ }^{\dagger}$ \\
\hline Orchidaceae & Campylocentrum crassyrhyzum* & Bromeliaceae & Tillandsia anceps ${ }^{\dagger}$ \\
\hline Orchidaceae & Catasetum macrocarpum & Bromeliaceae & Tillandsia bulbosa $a^{\dagger}$ \\
\hline Orchidaceae & Cattleya granulosa & Bromeliaceae & Vriesea gladioliflora $^{\dagger}$ \\
\hline Orchidaceae & Cattleya labiata ${ }^{*}$ & Bromeliaceae & Vriesea sanguinolenta \\
\hline Orchidaceae & Dichaea panamensis* & Cactaceae & Epiphyllum phyllanthus ${ }^{\dagger}$ \\
\hline Orchidaceae & Dimerandra emarginata* & Cactaceae & Hylocereus monacanthus ${ }^{\dagger}$ \\
\hline Orchidaceae & Encyclia longifolia & Cactaceae & Weberocereus tunilla $^{\dagger}$ \\
\hline Orchidaceae & Epidendrum difforme* & Gesneriaceae & Codonanthe macradenia ${ }^{\dagger}$ \\
\hline Orchidaceae & Epidendrum nocturnum* & Gesneriaceae & Columnea billbergiana \\
\hline Orchidaceae & Epidendrum ramosum* ${ }^{*}$ & Gesneriaceae & Drymonia serrulata \\
\hline Orchidaceae & Epidendrum riggidum & Hymenophyllaceae & Hymenophyllum brevifrons \\
\hline Orchidaceae & Heterotaxis discolor & Hymenophyllaceae & Trichomanes anadromum $^{\dagger}$ \\
\hline Orchidaceae & Jacquiniella globosa & Hymenophyllaceae & ${\text { Trichomanes angustifrons }{ }^{\dagger}}^{\dagger}$ \\
\hline Orchidaceae & Maxillaria ochroleuca & Hymenophyllaceae & Trichomanes godmanii ${ }^{\dagger}$ \\
\hline Orchidaceae & Notylia lyrata & Hymenophyllaceae & Trichomanes nummularium ${ }^{\dagger}$ \\
\hline Orchidaceae & Gomesa barbata* & Hymenophyllaceae & Trichomanes ovale $^{\dagger}$ \\
\hline Orchidaceae & Polystachya concreta* & Hymenophyllaceae & Trichomanes punctatum ${ }^{\dagger}$ \\
\hline Orchidaceae & Prosthechea alagoensis & Lomariopsidaceae & Elaphoglossum herminieri \\
\hline Orchidaceae & Prosthechea fragrans & Lomariopsidaceae & Elaphoglossum sporadolepis $^{\dagger}$ \\
\hline Orchidaceae & Rodrighezia bahiensis* & Orchidaceae & Acianthera verecunda $a^{\dagger}$ \\
\hline Orchidaceae & Scaphyglottis emarginata & Orchidaceae & Aspasia principissa \\
\hline Orchidaceae & Scaphyglottis fusiformis* & Orchidaceae & Camaridium sp. \\
\hline Orchidaceae & Scaphyglottis sickii* & Orchidaceae & Campylocentrum micranthum ${ }^{\dagger}$ \\
\hline Orchidaceae & Stellis clorantha & Orchidaceae & Catasetum viridiflavum $^{\dagger}$ \\
\hline Orchidaceae & Stellis filiformis & Orchidaceae & Caularthron bilamellatum ${ }^{\dagger}$ \\
\hline Orchidaceae & Trichocentrum fuscum* & Orchidaceae & Christensonella uncata ${ }^{\dagger}$ \\
\hline Orchidaceae & Trigonidium acuminatum & Orchidaceae & Cochleanthes lipscombiae \\
\hline Piperaceae & Peperomia aff. circinata & Orchidaceae & Cryptarrhena guatemalensis \\
\hline Piperaceae & Peperomia macrostachya & Orchidaceae & ${\text { Dichaea panamensis }{ }^{\dagger}}^{\top}$ \\
\hline Piperaceae & Peperomia pellucida & Orchidaceae & Dimerandra emarginata ${ }^{\dagger}$ \\
\hline Piperaceae & Peperomia sp. & Orchidaceae & Elleanthus longibracteatus \\
\hline & & Orchidaceae & Epidendrum coronatum \\
\hline & & Orchidaceae & Epidendrum difforme ${ }^{\dagger}$ \\
\hline & & Orchidaceae & Epidendrum imatophyllum \\
\hline & & Orchidaceae & Epidendrum nocturnum \\
\hline & & Orchidaceae & Epidendrum rousseauae \\
\hline & & Orchidaceae & Epidendrum schlechterianum ${ }^{\dagger}$ \\
\hline & & Orchidaceae & Gongora quinquenervis ${ }^{\dagger}$ \\
\hline & & Orchidaceae & Heterotaxis discolor \\
\hline & & Orchidaceae & Heterotaxis sessilis \\
\hline & & Orchidaceae & Jacquiniella pedunculata ${ }^{\dagger}$ \\
\hline & & Orchidaceae & Jacquiniella sp. \\
\hline & & Orchidaceae & Kefersteinia sp. \\
\hline & & Orchidaceae & Lockhartia acuta ${ }^{\dagger}$ \\
\hline & & Orchidaceae & Lockhartia pittieri \\
\hline & & Orchidaceae & Macradenia brassavolae \\
\hline & & Orchidaceae & Masdevallia livingstoneana \\
\hline & & Orchidaceae & Maxillariella acervata \\
\hline & & Orchidaceae & Mormodes powellii \\
\hline & & Orchidaceae & Notylia albida ${ }^{\dagger}$ \\
\hline & & Orchidaceae & Oncidium lineoligerum \\
\hline & & Orchidaceae & Ornithocephalus sp. \\
\hline & & Orchidaceae & Polystachya foliosa ${ }^{\dagger}$ \\
\hline & & Orchidaceae & Specklinia brighamii ${ }^{\dagger}$ \\
\hline & & Orchidaceae & Specklinia grobyi ${ }^{\dagger}$ \\
\hline & & Orchidaceae & Prosthechea aemula \\
\hline & & Orchidaceae & Prosthechea chacaoensis \\
\hline & & Orchidaceae & Prosthechea chimborazoensis \\
\hline & & Orchidaceae & Rossioglossum ampliatum ${ }^{\dagger}$ \\
\hline & & Orchidaceae & Scaphyglottis behrii ${ }^{\dagger}$ \\
\hline & & Orchidaceae & Scaphyglottis longicaulis ${ }^{\dagger}$ \\
\hline & & Orchidaceae & Scaphyglottis prolifera $^{\dagger}$ \\
\hline & & Orchidaceae & Sobralia fenzliana \\
\hline & & Orchidaceae & Sobralia fragans ${ }^{\dagger}$ \\
\hline & & Orchidaceae & Stelis crescentiicola ${ }^{\dagger}$ \\
\hline & & Orchidaceae & Trichocentrum capistratum \\
\hline & & Orchidaceae & Trichopilia maculata $^{\dagger}$ \\
\hline
\end{tabular}




\begin{tabular}{|c|c|}
\hline Orchidaceae & Trichosalpinx orbicularis \\
\hline Orchidaceae & Trigonidium egertonianum \\
\hline Piperaceae & Peperomia cordulata ${ }^{\dagger}$ \\
\hline Piperaceae & Peperomia ebingeri \\
\hline Piperaceae & Peperomia macrostachya \\
\hline Piperaceae & Peperomia obtusifolia \\
\hline Piperaceae & Peperomia rotundifolia ${ }^{\dagger}$ \\
\hline Polypodiaceae & Campyloneurum aphanophlebium ${ }^{\dagger}$ \\
\hline Polypodiaceae & Campyloneurum phylitidis ${ }^{\dagger}$ \\
\hline Polypodiaceae & Dicranoglossum panamense ${ }^{\dagger}$ \\
\hline Polypodiaceae & 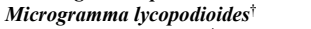 \\
\hline Polypodiaceae & Microgramma percussa ${ }^{\dagger}$ \\
\hline Polypodiaceae & Microgramma reptans \\
\hline Polypodiaceae & Niphidium crassifolium ${ }^{\dagger}$ \\
\hline Polypodiaceae & Pecluma pectinata \\
\hline Polypodiaceae & Serpocaulon triseriale \\
\hline Polypodiaceae & Serpocaulon wagneri \\
\hline Selaginellaceae & Huperzia dichotoma \\
\hline Vittariaceae & Ananthacorus angustifolius ${ }^{\dagger}$ \\
\hline Vittariaceae & Anetium citrifolium $^{\dagger}$ \\
\hline Vittariaceae & Antrophyum lanceolatum ${ }^{\dagger}$ \\
\hline Vittariaceae & Hecistopteris pumila \\
\hline 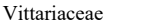 & \\
\hline
\end{tabular}




\section{Appendix}

Table B.3. Full transect data and comparisons between study sites. Several measures characterizing forest structure, branch abundance, as well as epiphyte abundance and richness were standardized per transect and their means were compared with simultaneous max- $t$ tests using Tukey contrasts that are robust under non-normality, heteroscedasticity and variable sample size.. The standardized measures \pm SD are given below. Significantly different means are followed by different letters representing pairwise differences. Additionally, total numbers per study site are provided. We show values with both transect and $\mathrm{m}^{2}$ as unit area. Note that for epiphytes, only mean values for Panamanian transects without ferns and aroids were used in the comparisons with Brazilian study sites. The symbol $\uparrow$ indicates $n=35$ (excluding one transect without trees).

\begin{tabular}{|c|c|c|c|c|c|}
\hline \multirow{2}{*}{$\begin{array}{l}\text { Variable } \\
\end{array}$} & & \multirow{2}{*}{$\begin{array}{c}\text { Brazilian core } \\
\text { transects } \\
(n=30)\end{array}$} & \multirow{2}{*}{$\begin{array}{c}\text { Brazilian } \\
\text { edge } \\
\text { transects } \\
(n=30)\end{array}$} & \multicolumn{2}{|c|}{ Panamanian transects $(n=36)$} \\
\hline & & & & No ferns and aroids & All species \\
\hline \multirow[t]{5}{*}{ Trees } & Total & 184 & 154 & 171 & \\
\hline & Mean number per transect & $5.1 \pm 2.1 \mathrm{ab}$ & $6.4 \pm 3.2 \mathrm{a}$ & $4.8 \pm 2.1 \mathrm{~b}$ & \\
\hline & Mean DBH (m) & $0.2 \pm 0.1 \mathrm{a}$ & $0.18 \pm 0.06 \mathrm{~b}$ & $0.16 \pm 0.07 \mathrm{~b} \dagger$ & \\
\hline & Mean height at first branching (m) & $8.2 \pm 2.0 \mathrm{a}$ & $4.7 \pm 2.0 \mathrm{~b}$ & $7.8 \pm 2.4 \mathrm{a} \dagger$ & \\
\hline & Mean height (m) & $15.4 \pm 3.4 \mathrm{a}$ & $10.9 \pm 2.7 b$ & $12.7 \pm 3.3 \mathrm{c} \dagger$ & \\
\hline \multirow[t]{3}{*}{ Branch abundance } & Total & 9759 & 6721 & 7939 & \\
\hline & Mean per transect & $325 \pm 284$ & $224 \pm 102$ & $220 \pm 169$ & \\
\hline & Mean per $\mathbf{m}^{2}$ & $130 \pm 114$ & $90 \pm 41$ & $88 \pm 68$ & \\
\hline \multirow[t]{6}{*}{ Epiphyte abundance } & Total & 546 & 349 & 164 & 232 \\
\hline & Mean per transect & $18.2 \pm 20.5 \mathrm{a}$ & $11.6 \pm 17.8 \mathrm{ab}$ & $4.6 \pm 7.1 \mathrm{~b}$ & $6.4 \pm 9.8$ \\
\hline & Mean per $\mathbf{m}^{2}$ & $0.36 \pm 0.41 \mathrm{a}$ & $0.23 \pm 0.36 \mathrm{ab}$ & $0.11 \pm 0.15 \mathrm{~b}$ & $0.13 \pm 0.20$ \\
\hline & Total adults & 211 & 153 & 86 & 101 \\
\hline & Mean adults per transect & $7.0 \pm 8.8 \mathrm{a}$ & $5.1 \pm 11.8 \mathrm{ab}$ & $2.4 \pm 4.1 \mathrm{~b}$ & $2.8 \pm 4.7$ \\
\hline & Mean adults per $\mathbf{m}^{2}$ & $0.14 \pm 0.18 \mathrm{a}$ & $0.10 \pm 0.24 \mathrm{ab}$ & $0.05 \pm 0.08 \mathrm{~b}$ & $0.06 \pm 0.09$ \\
\hline \multirow{6}{*}{$\begin{array}{l}\text { Epiphyte abundance } \\
\text { (detached from branches) }\end{array}$} & Total & 179 & 89 & 52 & 68 \\
\hline & Mean per transect & $6.0 \pm 4.9 \mathrm{a}$ & $3.0 \pm 7.2 \mathrm{ab}$ & $1.4 \pm 4.3 \mathrm{~b}$ & $1.9 \pm 6.0$ \\
\hline & Mean per $\mathbf{m}^{2}$ & $0.12 \pm 0.10 \mathrm{a}$ & $0.06 \pm 0.14 \mathrm{ab}$ & $0.03 \pm 0.09 \mathrm{~b}$ & $0.04 \pm 0.12$ \\
\hline & Total adults & 100 & 57 & 35 & 42 \\
\hline & Mean adults per transect & $3.3 \pm 3.2 \mathrm{a}$ & $1.9 \pm 6.2 \mathrm{ab}$ & $1.0 \pm 3.1 \mathrm{~b}$ & $1.2 \pm 3.8$ \\
\hline & Mean adults per $\mathbf{m}^{2}$ & $0.07 \pm 0.06 \mathrm{a}$ & $0.04 \pm 0.12 \mathrm{ab}$ & $0.02 \pm 0.06 \mathrm{~b}$ & $0.02 \pm 0.08$ \\
\hline \multirow{8}{*}{$\begin{array}{l}\text { Epiphyte abundance } \\
\text { (attached to branches) }\end{array}$} & Total & 367 & 260 & 112 & 164 \\
\hline & Mean per transect & $12.2 \pm 17.0 \mathrm{a}$ & $8.7 \pm 16.8 \mathrm{ab}$ & $3.1 \pm 5.1 \mathrm{~b}$ & $4.6 \pm 6.4$ \\
\hline & Mean per $\mathrm{m}^{2}$ & $0.24 \pm 0.34 \mathrm{a}$ & $0.17 \pm 0.34 \mathrm{ab}$ & $0.06 \pm 0.10 \mathrm{~b}$ & $0.09 \pm 0.13$ \\
\hline & Mean per transect per branch & $2.810^{-3} \pm 3.510^{-3}$ & $2.210^{-3} \pm 4.510^{-3}$ & $1.110^{-3} \pm 2.010^{-3}$ & $1.810^{-3} \pm 3.110^{-3}$ \\
\hline & Total adults & 1111 & 96 & 51 & 59 \\
\hline & Mean adults per transect & $3.7 \pm 6.3$ & $3.20 \pm 10.3$ & $1.4 \pm 2.5$ & $1.6 \pm 2.5$ \\
\hline & Mean adults per $\mathbf{m}^{2}$ & $0.07 \pm 0.13$ & $0.06 \pm 0.20$ & $0.03 \pm 0.05$ & $0.03 \pm 0.05$ \\
\hline & Mean adults per transect per branch & $8.510^{-4} \pm 1.510^{-3}$ & $8.810^{-4} \pm 2.410^{-3}$ & $6.710^{-4} \pm 1.310^{-3}$ & $7.510^{-4} \pm 1.310^{-3}$ \\
\hline \multirow[t]{6}{*}{ Epiphyte richness (total) } & Total & 23 & 16 & 27 & 39 \\
\hline & Mean per transect & $5.1 \pm 3.2 \mathrm{a}$ & $2.3 \pm 1.9 \mathrm{~b}$ & $1.9 \pm 2.3 \mathrm{~b}$ & $2.8 \pm 3.6$ \\
\hline & Mean per $\mathbf{m}^{2}$ & $0.10 \pm 0.06 \mathrm{a}$ & $0.05 \pm 0.04 \mathrm{~b}$ & $0.04 \pm 0.05 \mathrm{~b}$ & $0.06 \pm 0.07$ \\
\hline & Total adults & 21 & 14 & 17 & 24 \\
\hline & Mean adults per transect & $3.3 \pm 2.7 \mathrm{a}$ & $1.1 \pm 1.3 \mathrm{~b}$ & $1.1 \pm 1.6 \mathrm{~b}$ & $1.4 \pm 2.0$ \\
\hline & Mean adults per $\mathbf{m}^{2}$ & $0.07 \pm 0.05 \mathrm{a}$ & $0.02 \pm 0.02 \mathrm{~b}$ & $0.02 \pm 0.03 \mathrm{~b}$ & $0.03 \pm 0.04$ \\
\hline \multirow{6}{*}{$\begin{array}{l}\text { Epiphyte richness } \\
\text { (detached from branches) }\end{array}$} & Total & 20 & 10 & 16 & 23 \\
\hline & Mean per transect & $3.0 \pm 2.4 \mathrm{a}$ & $0.7 \pm 1.1 \mathrm{~b}$ & $0.6 \pm 1.4 \mathrm{~b}$ & $0.9 \pm 2.4$ \\
\hline & Mean per $\mathbf{m}^{2}$ & $0.06 \pm 0.05 \mathrm{a}$ & $0.015 \pm 0.02 \mathrm{~b}$ & $0.01 \pm 0.03 b$ & $0.02 \pm 0.05$ \\
\hline & Total adults & 18 & 9 & 10 & 13 \\
\hline & Mean adults per transect & $2.3 \pm 1.9 \mathrm{a}$ & $0.4 \pm 0.7 \mathrm{~b}$ & $0.4 \pm 1.0 \mathrm{~b}$ & $0.6 \pm 1.5$ \\
\hline & Mean adults per $\mathbf{m}^{2}$ & $0.05 \pm 0.04 \mathrm{a}$ & $0.007 \pm 0.014 \mathrm{~b}$ & $0.008 \pm 0.02 b$ & $0.01 \pm 0.03$ \\
\hline \multirow{8}{*}{$\begin{array}{l}\text { Epiphyte richness } \\
\text { (attached to branches) }\end{array}$} & Total & 17 & 13 & 18 & 29 \\
\hline & Mean per transect & $3.3 \pm 2.5 \mathrm{a}$ & $1.8 \pm 1.8 \mathrm{~b}$ & $1.4 \pm 1.6 \mathrm{~b}$ & $2.2 \pm 2.3$ \\
\hline & Mean per $\mathrm{m}^{2}$ & $0.06 \pm 0.05 \mathrm{a}$ & $0.38 \pm 0.35 b$ & $0.03 \pm 0.03 \mathrm{~b}$ & $0.04 \pm 0.05$ \\
\hline & Mean per transect per branch & $7.710^{-4} \pm 7.110^{-4}$ & $5.210^{-4} \pm 5.510^{-4}$ & $5.310^{-4} \pm 7.510^{-4}$ & $7.310^{-4} \pm 7.910^{-4}$ \\
\hline & Total adults & 16 & 11 & 12 & 18 \\
\hline & Mean adults per transect & $1.57 \pm 2.0$ & $0.80 \pm 1.14$ & $0.72 \pm 1.08$ & $0.94 \pm 1.24$ \\
\hline & Mean adults per $\mathbf{m}^{2}$ & $0.03 \pm 0.04$ & $0.015 \pm 0.02$ & $0.014 \pm 0.02$ & $0.019 \pm 0.02$ \\
\hline & Mean adults per transect per branch & $3.310^{-4} \pm 4.610^{-4}$ & $1.910^{-4} \pm 3.010^{-4}$ & $2.810^{-4} \pm 4.510^{-4}$ & $3.210^{-4} \pm 4.210^{-4}$ \\
\hline
\end{tabular}


Table B.4. Generalized additive mixed-effects models (GAMMs) investigating the influence of branch diameter on different variables. The table provides the effective degrees of freedom ( $\left.\mathrm{df}_{\text {eff }}\right)$ for both fixed (diameter class) and random (transect) effects as well as the trend of the relationship over branch diameter. The $\mathrm{df}_{\text {eff }}$ reflect the ruggedness of the smoothing parameter. For absolute branch abundance (branches per transect), epiphyte abundance (individuals per transect), richness (species per transect) and proportion of adults, all transects were considered ( $\mathrm{n}=30,30$ and 36 for Brazilian core, Brazilian edge and Panamanian transects, respectively), whereas for epiphyte abundance and richness per branch, only transects with epiphytes were considered $(n=26,21$ and 25 for Brazilian core, Brazilian edge and Panamanian transects, respectively). Significant P-values $(\alpha=5 \%)$ are indicated with asterisks.

\begin{tabular}{|c|c|c|c|c|c|c|c|c|}
\hline Study site & Effect & & $\begin{array}{r}\text { Branch } \\
\text { abundanc } \\
\mathrm{e} \\
\end{array}$ & $\begin{array}{r}\text { Abs. epiphyte } \\
\text { abundance }\end{array}$ & $\begin{array}{r}\text { Abs. } \\
\text { epiphyte } \\
\text { richness }\end{array}$ & $\begin{array}{r}\text { Epiphyte } \\
\text { abundance/ } \\
\text { branch }\end{array}$ & $\begin{array}{r}\text { Epiphyte } \\
\text { richness/ } \\
\text { branch }\end{array}$ & $\begin{array}{r}\text { Proportion } \\
\text { of adults }\end{array}$ \\
\hline \multirow[t]{3}{*}{ Brazil core } & \multirow{2}{*}{$\begin{array}{l}\text { Diameter } \\
\text { class }\end{array}$} & $\mathbf{d f}_{\text {eff }}$ & 1.00 & 1.98 & 1.96 & 1.00 & 1.00 & 1.00 \\
\hline & & Trend & negative $e^{* * *}$ & unimodal $* * *$ & unimodal $* * *$ & positive $\mathrm{e}^{* * *}$ & positive $e^{* * *}$ & positive $* * *$ \\
\hline & Transect & $\mathbf{d} \mathbf{f}_{\text {eff }}$ & 0 & $25^{* * *}$ & $23^{* * *}$ & $9 *$ & 8 & $10^{* * *}$ \\
\hline \multirow[t]{3}{*}{ Brazil edge } & \multirow{2}{*}{$\begin{array}{l}\text { Diameter } \\
\text { class }\end{array}$} & $\mathbf{d f}_{\text {eff }}$ & 1.89 & 1.00 & 1.94 & 1.00 & 1.00 & 1.00 \\
\hline & & Trend & negative ${ }^{* * *}$ & none & unimodal $* * *$ & positive $\mathrm{e}^{* * *}$ & positive $* * *$ & positive * \\
\hline & Transect & $\mathbf{d f}_{\text {eff }}$ & 0 & $26 * * *$ & $19^{* * *}$ & 5 & $6^{*}$ & $13^{* * *}$ \\
\hline \multirow[t]{3}{*}{ Panama } & \multirow{2}{*}{$\begin{array}{l}\text { Diameter } \\
\text { class }\end{array}$} & $\mathbf{d f}_{\text {eff }}$ & 1.00 & 1.87 & 1.91 & 1.54 & 1.64 & 1.60 \\
\hline & & Trend & negative*** & positive*** & positive*** & positive*** & positive*** & positive $* * *$ \\
\hline & Transect & $\mathbf{d f}_{\text {eff }}$ & 0.84* & $28 * * *$ & $22 * * *$ & $8 *$ & $8^{*}$ & $4 *$ \\
\hline
\end{tabular}

Table B.5. Indicator species for the compositional difference between ground and canopy at Panamanian transects. We performed a Dufrene-Legendre indicator species analysis to assess the species that contributed to the significant difference in species composition between the epiphytes found on the forest floor and in the canopy. All listed species were indicatory of canopy composition in the analysis considering all epiphytes (including epiphytes found on the forest floor detached from branches and in the entire canopy). Indicator species resulting from the analysis considering only epiphytes on substrate $<10 \mathrm{~cm}$ in diameter are indicated in bold.

\begin{tabular}{ll}
\hline Family & Species \\
\hline Araceae & Anthurium acutangulum \\
& Anthurium clavigerum \\
& Anthurium friedrichsthalii \\
& Anthurium hacumense \\
Bromeliaceae & Vriesea gladioliflora \\
Hymenophyllaceae & Trichomanes angustifrons \\
& Trichomanes nummularium \\
& Trichomanes ovale \\
& Trichomanes punctatum \\
Lomariopsidaceae & Elaphoglossum sporadolepis \\
Orchidaceae & Dichaea panamensis \\
& Polystachya foliosa \\
Polypodiaceae & Scaphyglottis longicaulis \\
& Campyloneurum aphanophlebium \\
& Campyloneurum phylitidis \\
Vittariaceae & Dicranoglossum panamense \\
& Niphidium crassifolium \\
& Ananthacorus angustifolius \\
& Anetium citrifolium \\
\hline
\end{tabular}




\section{Supplementary information to chapter 4}

(a)

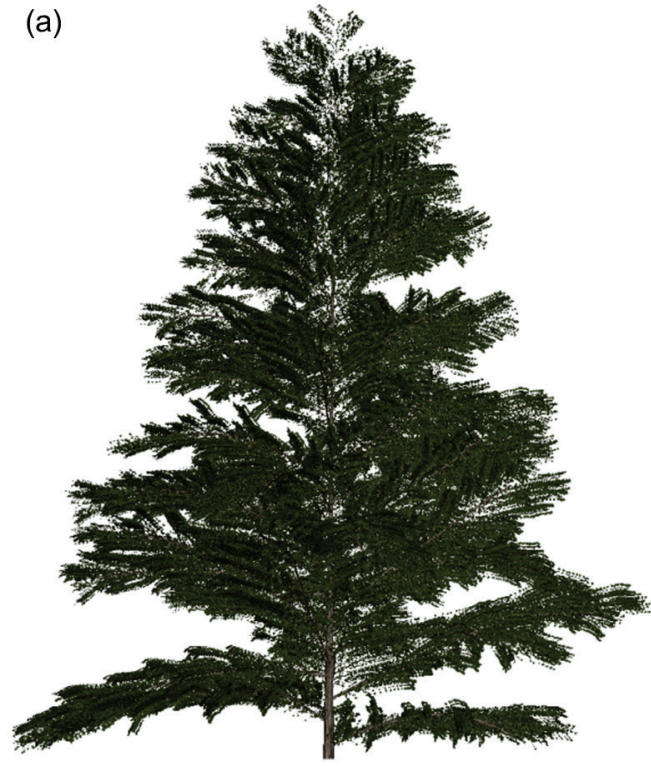

(b)

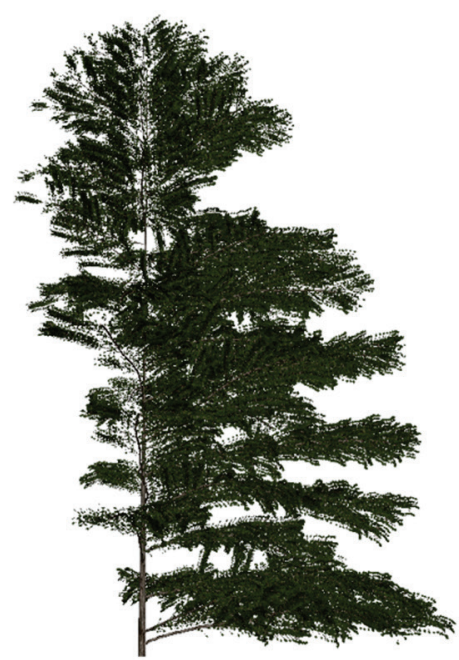

(c)

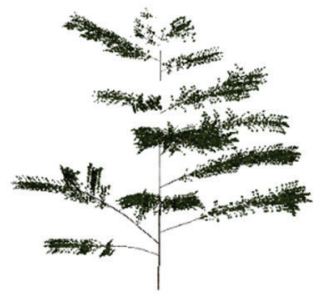

Figure C.1. Effects of competition on tree growth. Three trees with identical functional and structural traits were simulated (a) without competition, (b) with one-sided competition and (c) with competition from 8 surrounding trees. 
Table C.1. List of abbreviations used in this study.

\begin{tabular}{|c|c|c|}
\hline Symbol & Explanation & Unit \\
\hline$A_{B}$ & Cross-sectional area of branch & $\mathrm{cm}^{2}$ \\
\hline$A_{\mathrm{L}}$ & Leaf area in leaf compartment & $\mathrm{cm}^{2}$ \\
\hline$A_{\text {LMax }}$ & Maximum leaf area in voxel & $\mathrm{cm}^{2}$ \\
\hline$A_{\text {LProd }}$ & Leaf area produced in leaf compartment in one year & $\mathrm{cm}^{2}$ \\
\hline$A_{\text {LProdMax }}$ & Absolute maximum leaf area production per leaf compartment & $\mathrm{cm}^{2}$ \\
\hline ALProdMaxMethod & $\begin{array}{l}\text { Parameter specifying whether an invariable (ALProdMaxMethod }=0 \text { ) or a variable } \\
\left.A_{\text {LProdMax }} \text { is used (ALProdMaxMethod }=1\right) .\end{array}$ & - \\
\hline$A_{\text {LProdTheo }}$ & Theoretical maximum annual leaf area production per leaf compartment & $\mathrm{cm}^{2}$ \\
\hline$A_{\text {LProdTot }}$ & Total leaf area production in leaf compartment & $\mathrm{cm}^{2}$ \\
\hline$A_{\text {LSum }}$ & Total leaf area associated with growing branch & $\mathrm{cm}^{2}$ \\
\hline$A_{\text {LTot }}$ & Total leaf area in voxel $\left(\right.$ per $\left.\mathrm{m}^{2}\right)$ & $\mathrm{cm}^{2} \mathrm{~m}^{-2}$ \\
\hline$A_{S}$ & Cross-sectional area of branch segment & $\mathrm{cm}^{2}$ \\
\hline$A_{\text {Sec }}$ & Cross-sectional area of branch section & $\mathrm{cm}^{2}$ \\
\hline$A_{T}$ & Cross-sectional area of trunk & $\mathrm{cm}^{2}$ \\
\hline$B_{\mathrm{L}}$ & Leaf dry mass (in leaf compartment) & $\mathrm{g}$ \\
\hline$B_{\text {LInit }}$ & Initial leaf biomass of new leaf compartment & g \\
\hline$B_{\text {LMin }}$ & Minimum leaf biomass below which leaf compartment is removed & g \\
\hline$B_{\text {LProd }}$ & Effective leaf biomass production in leaf compartment & $\mathrm{g}$ \\
\hline$B_{\text {LProdPot }}$ & Potential leaf biomass production when using the effective growth rate $G R$ & $\mathrm{~g}$ \\
\hline$B_{\text {LProdTheo }}$ & Theoretical maximum annual leaf dry mass production per leaf compartment & g \\
\hline BrCollide & $\begin{array}{l}\text { Parameter specifying whether branches stop to grow in length if the collide with } \\
\text { surrounding trees }(\text { BrCollide }=1) \text { or not }(\text { BrCollide }=0)\end{array}$ & - \\
\hline BrMortMethod & $\begin{array}{l}\text { Parameter specifying whether branches are removed only if the lost all leaf } \\
\text { compartments }(\text { BrMortMethod }=0 \text { ), or if they are additionally removed randomly } \\
\text { (BrMortMethod }=1 \text { ) or based on their biomass (BrMortMethod }=2 \text { ) }\end{array}$ & - \\
\hline$C$ & Relative contribution of voxel in average light intensity calculations & - \\
\hline$C_{0}$ & Carbon overhead costs & - \\
\hline$C_{B}$ & Amount of leaf dry mass that can be produced per unit of assimilated carbon & $\mathrm{g} \mathrm{gC}^{-1}$ \\
\hline$C B L_{\text {ratio }}$ & C-mass to biomass ratio of leaves & $\mathrm{gC} \mathrm{g}^{-1}$ \\
\hline$C B W_{\text {ratio }}$ & C-mass to biomass ratio of wood & $\mathrm{gC} \mathrm{g}^{-1}$ \\
\hline$C_{\text {gross }}$ & Gross carbon assimilation rate per unit of leaf dry mass & $\mathrm{gC} \mathrm{g}^{-1} \mathrm{~d}^{-1}$ \\
\hline$C_{n e t}$ & Net carbon assimilation rate per unit of leaf dry mass & $\mathrm{gC} \mathrm{g}^{-1} \mathrm{~d}^{-1}$ \\
\hline$C R_{r}$ & Crown radius of tree & $\mathrm{cm}$ \\
\hline$D_{\mathrm{B}}$ & Diameter of branch & $\mathrm{cm}$ \\
\hline$D_{\text {BLat }}$ & Diameter of lateral branch & $\mathrm{cm}$ \\
\hline$D_{\text {ini }}$ & Initial diameter of seedling (fixed value) & $\mathrm{cm}$ \\
\hline$D I_{\mathrm{S}}$ & Distance of branch segment to its branch base & $\mathrm{cm}$ \\
\hline$D_{\mathrm{NMin}}$ & Trees with a diameter $>D_{\mathrm{NMin}}$ can create gaps (if TrMortNeight $=1$ ) & $\mathrm{cm}$ \\
\hline$D_{\mathrm{S}}$ & Diameter of branch segment & $\mathrm{cm}$ \\
\hline$D_{\mathrm{Sec}}$ & Diameter of branch section & $\mathrm{cm}$ \\
\hline$D_{\mathrm{T}}$ & Diameter of trunk & $\mathrm{cm}$ \\
\hline EdgeC & $\begin{array}{l}\text { Parameter specifying whether a forest fragment with a real edge }(E d g e C=1) \text { or a forest } \\
\text { patch within a forest matrix }(E d g e C=0) \text { is simulated }\end{array}$ & - \\
\hline$F_{\text {Dist }}$ & Frequency of disturbances (average number of years between two events) & a \\
\hline$G_{\max }$ & Maximum gross photosynthetic rate (g C per g dry mass per day) & $\mathrm{gC} \mathrm{g}^{-1} \mathrm{~d}^{-1}$ \\
\hline GR & Effective growth rate of leaf compartment & $\mathrm{d}^{-1}$ \\
\hline$G R_{\max }$ & Maximum growth rate of leaf compartment (considering $A_{\mathrm{LMax}}$ ) & $d^{-1}$ \\
\hline$G R_{\text {pot }}$ & Potential growth rate of leaf compartment & $\mathrm{d}^{-1}$ \\
\hline$h_{\text {sun }}$ & Assumed number of sun hours per day & $\mathrm{h}$ \\
\hline I & Effective light intensity in voxel & $\mu \mathrm{mol} \mathrm{m} \mathrm{m}^{-2} \mathrm{~s}^{-1}$ \\
\hline$I_{\mathrm{M}}$ & Light intensity at apical meristem & $\mu \mathrm{mol} \mathrm{m} \mathrm{m}^{-2}$ \\
\hline$I_{\max }$ & Light intensity above canopy & $\mu \mathrm{mol} \mathrm{m} \mathrm{m}^{-2} \mathrm{~s}^{-1}$ \\
\hline$I_{S C}$ & Single column light intensity & $\mu \mathrm{mol} \mathrm{m} \mathrm{m}^{-2} \mathrm{~s}^{-1}$ \\
\hline$I_{\mathrm{T}}$ & Light intensity threshold regulating apical dominance of trunk apical meristem & $\mu \mathrm{mol} \mathrm{m} \mathrm{s}^{-2}$ \\
\hline$k$ & Light intensity at which the gross photosynthetic rate is half of its maximum & $\mu \mathrm{mol} \mathrm{m} \mathrm{m}^{-2} \mathrm{~s}^{-1}$ \\
\hline
\end{tabular}


$k_{\text {int }}$

$k_{\mathrm{L}}$

LAI

$L_{\mathrm{B}}$

$L_{\text {Cor }}$

$L_{C r}$

$L D_{\mathrm{B}}$

$L D_{C}$

$L D_{T}$

$L_{I B}$

$L_{\text {IBMax }}$

$L_{\text {IBMin }}$

LightC

$L_{\text {inc }}$

$L_{I T}$

$L_{\text {ITMax }}$

$L_{\text {ITMin }}$

$L L$

$L_{\mathrm{P}}$

$L_{\text {PMax }}$

$L_{\text {PMaxAbs }}$

$L P_{\text {ratio }}$

$L_{\mathrm{PS}}$

$L R$

$L_{\mathrm{S}}$

$L_{\text {SFirst }}$

$L_{\text {SLast }}$

$L_{T}$

$L_{T C r}$

$L_{\text {TMax }}$

$L_{\text {TRg }}$

$L_{\mathrm{V}}$

$\operatorname{Max} X$

$\operatorname{Max} Y$

MaxZ

$m_{\text {B }}$

$m_{B B}$

$m_{\mathrm{BR}}$

$M_{B S}$

$m_{\text {Dist }}$

$m_{\text {Neigh }}$

$m_{\mathrm{T}}$

$m_{T B}$

$M_{\mathrm{TS}}$

$n_{\text {BLat }}$

$n_{B S e g}$

$N_{\text {mass }}$

$n_{\text {Seed }}$

$n_{\text {Spec }}$

$n_{\mathrm{V}}$

$O_{\text {B }}$

$P_{\text {BEnd }}$ XYZ

$P_{\mathrm{BStart}} \mathrm{XYZ}$

$P h_{\mathrm{FO}}$

$P_{\mathrm{LC}}{ }^{\mathrm{XYZ}}$

Factor controlling the relationship between internode length and total annual length growth

Light extinction coefficient (Lambert-Beer equation)

Leaf area index

Length of branch

Width of corridor around core model area

Critical length for buckling

Allometric parameter of length-diameter relationship of branches

Allometric parameter of length-diameter relationship (critical shape parameter)

Allometric parameter of length-diameter relationship of trunks

Branch internode length

Species-specific maximum branch internode length

Species-specific minimum branch internode length

Parameter specifying method to calculate average light intensity; LightC $=[1,2,3]$

Relative intensification in height growth (apical dominance)

Trunk internode length

Species-specific maximum trunk internode length

Species-specific minimum trunk internode length

Leaf lifespan

Length of pipe connected with leaf compartment (corrected after apical control)

Maximum pipe length (under given $S I$ )

Maximum pipe length (theoretical maximum when $S I=1$ )

Ratio between leaf area an pipe cross-sectional area

Length of pipe connected with leaf compartment

Maximal distance of surrounding voxels to be considered in light calculation

Length of branch segment

Length of the first branch segment in each year

Length of the last branch segment in each year

Length of trunk

Critical trunk length

Maximum trunk length under consideration of $S T$

Trunk length following unrestricted regular growth mechanism

Side length of voxels

Spatial extent of core model area (in $\mathrm{X}$ direction)

Spatial extent of core model area (in Y direction)

Spatial extent of core model area (in $\mathrm{Z}$ direction)

Biomass-based branch mortality rate (if BrMortMethod=2)

Parameter of biomass-based branch mortality rate (if BrMortMethod=2)

Random branch mortality rate (if BrMortMethod=1)

Scaling exponent in biomass-based branch mortality rate (if BrMortMethod=2)

Average relative mortality rate in a disturbance event (if TrMortDist $=1$ )

Trees affected by falling trees die with a probability of $m_{\text {Neigh }}$

Biomass-based tree mortality rate

Parameter of biomass-based tree mortality rate

Scaling exponent in biomass-based tree mortality rate

Number of new lateral branches of a single branch in one time step

Number of new branch segments of a single branch in one time step

Nitrogen concentration

Number of seedlings dispersed at each time step

Number of species in species list

Number of new voxel a branch is intersecting with

Branch order

End position of branch (in $\mathrm{X}, \mathrm{Y}$ and $\mathrm{Z}$ direction)

Start position of branch (in $\mathrm{X}, \mathrm{Y}$ and $\mathrm{Z}$ direction)

Number of first order branches arranged in a $360^{\circ}$ circle

Position of leaf compartment (in $\mathrm{X}, \mathrm{Y}$ and $\mathrm{Z}$ direction)

$\mathrm{m}$

$\mathrm{cm}$

$-$

$-$

-

$\mathrm{cm}$

$\mathrm{cm}$

-

$\mathrm{cm}$ 


\begin{tabular}{|c|c|c|}
\hline$P_{\mathrm{M}}^{\mathrm{XYZ}}$ & Position of apical meristem (in $\mathrm{X}, \mathrm{Y}$ and $\mathrm{Z}$ direction) & $\mathrm{cm}$ \\
\hline$P_{R U}$ & Pipe-reuse factor & - \\
\hline$P_{\text {SEnd }} \mathrm{XYZ}$ & End position of branch segment (in $\mathrm{X}, \mathrm{Y}$ and $\mathrm{Z}$ direction) & $\mathrm{cm}$ \\
\hline$P_{\text {SStart }}{ }^{\mathrm{XYZ}}$ & Start position of branch segment (in $\mathrm{X}, \mathrm{Y}$ and $\mathrm{Z}$ direction) & $\mathrm{cm}$ \\
\hline$P_{\mathrm{T}}^{\mathrm{XY}}$ & Position of trunk (in $\mathrm{X}$ and $\mathrm{Y}$ direction) & $\mathrm{cm}$ \\
\hline$R_{\mathrm{L}}$ & Respiration rate per gram of leaf dry mass & $\mathrm{gC} \mathrm{g}^{-1} \mathrm{~d}^{-1}$ \\
\hline$R_{\mathrm{w}}$ & Respiration rate per gram of sapwood & $\mathrm{gC} \mathrm{g}^{-1} \mathrm{~d}^{-1}$ \\
\hline$R_{\mathrm{WTot}}$ & Respiration rate of pipes per gram of leaf dry-mass & $\mathrm{gC} \mathrm{g}^{-1} \mathrm{~d}^{-1}$ \\
\hline$S_{\mathrm{F}}$ & Factor regulating the shortening of branches with their order & - \\
\hline$S I$ & Site index describing the relative quality of the forest patch & - \\
\hline$S L A$ & Specific leaf area & $\mathrm{cm}^{2} \mathrm{~g}^{-1}$ \\
\hline$S T$ & Safety factor for trunk growth. & - \\
\hline Stochasticity & $\begin{array}{l}\text { Parameter specifying whether stochastic variations of structural traits are simulated } \\
(\text { Stochasticity }=1) \text { or not (Stochasticity }=0)\end{array}$ & - \\
\hline$S_{\text {Trop }}$ & Strength of tropism (negative values: phototropism; positive: gravitropism) & - \\
\hline $\mathrm{St}_{\text {Trop }}$ & Maximum deviation from $S_{\text {Trop }}$ (if Stochasticity $=1$ ) & - \\
\hline $\mathrm{St}_{\mathrm{Tw}}$ & Maximal rotation along the main growth axis (if Stochasticity $=1$ ) & $\circ$ \\
\hline $\mathrm{St}_{\mathrm{aSFO}}$ & Maximum deviation from $\alpha_{\mathrm{SFO}}$ (if Stochasticity $=1$ ) & $\circ$ \\
\hline $\mathrm{St}_{\alpha \mathrm{TFO}}$ & Maximum deviation from $\alpha_{\mathrm{TFO}}$ (if Stochasticity $=1$ ) & $\circ$ \\
\hline $\mathrm{St}_{\alpha \mathrm{TSO}}$ & Maximum deviation from $\alpha_{\mathrm{TSO}}$ (if Stochasticity $=1$ ) & $\circ$ \\
\hline$t_{\max }$ & Number of simulated annual time steps & a \\
\hline$t_{p}$ & Productive time period of leaf compartment during one year & $\mathrm{d}$ \\
\hline TrMortDist & Parameter specifying if tree mortality due to disturbances is simulated (TrMortDist $=1$ ) & - \\
\hline TrMortNeigh & $\begin{array}{l}\text { Parameter specifying if tree mortality due to falling neighboring trees is simulated } \\
(\text { TrMortNeight=1) }\end{array}$ & - \\
\hline$t_{\text {year }}$ & Number of days per year suitable for photosynthesis & $\mathrm{d}$ \\
\hline$\alpha_{\mathrm{SFO}}$ & Angle of first order branches from side view & $\circ$ \\
\hline$\alpha_{\text {TFO }}$ & Angle between first order branches from top view & $\circ$ \\
\hline$\alpha_{\text {TSO }}$ & Angle between second and first order branch from top view & $\circ$ \\
\hline$\beta_{\mathrm{D}}$ & Maximum relative increase in height growth when $I_{\mathrm{M}}<I_{\mathrm{T}}$ & - \\
\hline$\beta_{\mathrm{S}}$ & Shape parameter regulating apical dominance of trunk apical meristem & - \\
\hline$\triangle L_{B P o t}$ & Potential length increase of branch & $\mathrm{cm} \mathrm{a}^{-1}$ \\
\hline$\Delta L_{T P O t}$ & Effective potential length increase of trunk & $\mathrm{cm} \mathrm{a}^{-1}$ \\
\hline$\Delta L_{T P o t C r}$ & Potential length increase of trunk up to the critical buckling length & $\mathrm{cm} \mathrm{a}^{-1}$ \\
\hline$\Delta L_{\text {TPotMax }}$ & Potential length increase of trunk up to the maximum tree height & $\mathrm{cm} \mathrm{a}^{-1}$ \\
\hline$\Delta L_{\text {TPotRg }}$ & Potential length increase of trunk when considering apical dominance & $\mathrm{cm} \mathrm{a}^{-1}$ \\
\hline$\rho_{\mathrm{W}}$ & Wood density & $\mathrm{g} \mathrm{cm}^{-3}$ \\
\hline
\end{tabular}


Table C.2. Parameters of the global file. The global file is a text file located in the main model folder and contains a set of parameters defining the basic set up of the model, such as the spatial extent of the number of time steps to be simulated.

\begin{tabular}{|c|c|c|c|}
\hline Parameter & Explanation & Unit & Symbol \\
\hline Timesteps & Number of simulated annual time steps & a & $t_{\max }$ \\
\hline Replicates & Number of replicates to be simulated & - & - \\
\hline $\operatorname{Max} X$ & Spatial extent of core model area (in X direction) & $\mathrm{m}$ & $\operatorname{Max} X$ \\
\hline $\operatorname{Max} Y$ & Spatial extent of core model area (in Y direction) & $\mathrm{m}$ & $\operatorname{Max} Y$ \\
\hline $\operatorname{Max} Z$ & Spatial extent of core model area (in $\mathrm{Z}$ direction) & $\mathrm{m}$ & $\operatorname{Max} Z$ \\
\hline WidthCorridor & $\begin{array}{l}\text { Width of corridor around core model area (only integer } \\
\text { values allowed) }\end{array}$ & $\mathrm{m}$ & $L_{C o r}$ \\
\hline VoxelSize & Side length of voxels (only integer values allowed) & $\mathrm{m}$ & $L_{\mathrm{V}}$ \\
\hline ReportForest & Time interval in which forest variables are saved & a & - \\
\hline ReportLight & Time interval in which light variables are saved & a & - \\
\hline ReportMortality & Time interval in which mortality variables are saved & a & - \\
\hline ReportShoots & Time interval in which shoot variables are saved & a & - \\
\hline ReportTrees & Time interval in which trees variables are saved & a & - \\
\hline ReportVoxel & Time interval in which voxel variables are saved & a & - \\
\hline SimulateForest & $\begin{array}{l}\text { Parameter specifying whether a forest (SimulateForest }=1 \text { ) } \\
\text { or an individual tree (SimulateForest }=0) \text { is simulated }\end{array}$ & $\mathrm{a}$ & - \\
\hline ThreadCount & Number of threads that are used in parallel in light model & - & - \\
\hline VisualizationShader & $\begin{array}{l}\text { Parameter specifying whether rendered trees are shown } \\
(\text { VisualizationShader }=1) \text { or not }(\text { VisualizationShader }=0)\end{array}$ & - & - \\
\hline VisualizationMethod & Parameter specifying visualization method & - & - \\
\hline
\end{tabular}


Table C.3. Export parameters of the model. This table contains all parameters that are saved in each of the six different types of result files (Forest, Mortality, Shoots, Species, Trees and Voxels). The time interval at which each of this result files shall be saved to the hard disk can be defined by the user in the global file (Table C.2).

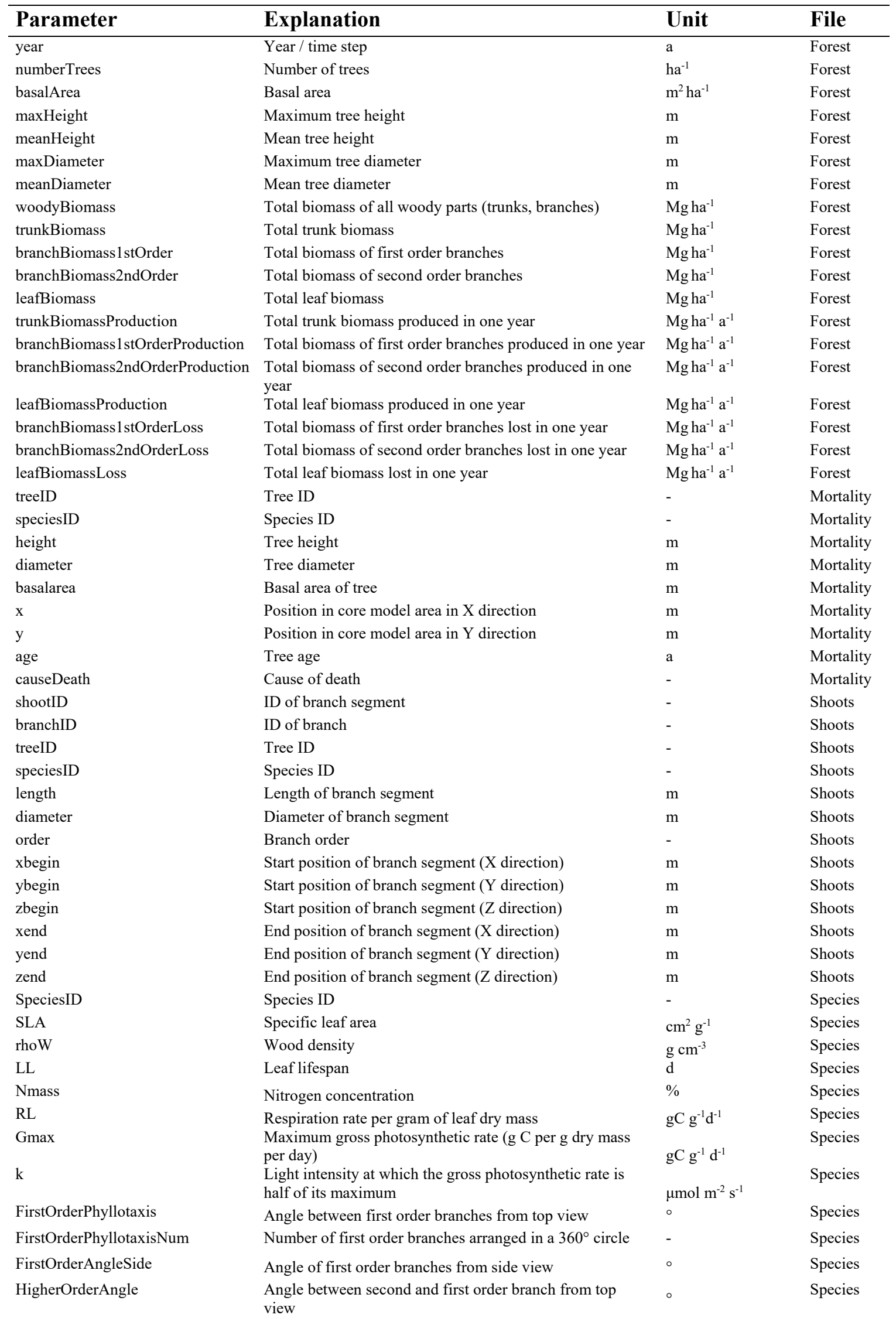




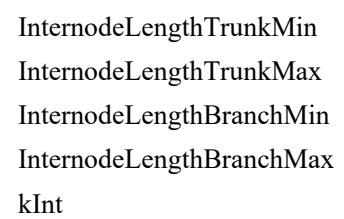

Species-specific minimum trunk internode length

Species-specific maximum trunk internode length

Species-specific minimum branch internode length

Species-specific maximum branch internode length

Strength of tropism (negative values: phototropism;

Height increase in one time step

Relative height increase in one time step

Diameter increase in one time step

Relative diameter increase in one time step

Basal area increase in one time step

Relative Basal area increase in one time step

Biomass of all woody tree parts (trunk and branches)

Biomass of trunk

Biomass of first order branches

Biomass of second order branches

Total leaf biomass of tree

Total leaf area of tree

Total trunk biomass produced in one year

Total biomass of first order branches produced in one year year

Total leaf biomass produced in one year

Total biomass of first order branches lost in one year

Total biomass of second order branches lost in one year

Total leaf biomass lost in one year

$\operatorname{Mgha}^{-1} \mathrm{a}^{-1} \quad$ Trees

Mg ha $^{-1} \mathrm{a}^{-1} \quad$ Trees

$\mathrm{Mgha}^{-1} \mathrm{a}^{-1} \quad$ Trees

Mgha $^{-1} \mathrm{a}^{-1} \quad$ Trees

Light conditions at apical stem meristem

Crown area of tree

Crown width of tree

Crown depth of tree

Crown width relative to tree height

Crown depth relative to tree height

Height of first branching

Position of Voxel (in X direction)

Position of Voxel (in Y direction)

Position of Voxel (in Z direction) Leaf area in Voxel
Species

Species

Species

Species

Species

Species

Species

Species

Species

Species

Species

Species

Species

Species

Species

Species

Species

Species

Trees

Trees

Trees

Trees

Trees

Trees

Trees

Trees

Trees

Trees

Trees

Trees

Trees

Trees

Trees

Trees

Trees

Trees

Trees

Trees

Trees

Trees

Trees

Trees

Trees

Trees

Trees

Trees

Trees

Trees

Trees

Voxels

Voxels

Voxels Voxels 
Table C.4. Parameters of the pass file. The pass file is a text file located in the main model folder and contains a set of parameters for each replicate. Each pass file includes global parameters, ranges of functional and structural traits, but also parameters to select a specific optional model mechanism. The parameter values shown in this table are the values of the model shown in the main manuscript.

\begin{tabular}{|c|c|c|c|c|}
\hline Parameter & Explanation & Unit & Symbol & Value \\
\hline LightC & Parameter specifying method to calculate average light intensity; Light $C=[1,2,3]$ & - & LightC & 1 \\
\hline ALMax & Maximum leaf area in voxel & $\mathrm{cm}^{2}$ & $A_{\text {LMax }}$ & 15000 \\
\hline ALProdMax (Min,Max) & Absolute maximum leaf area production per leaf compartment & $\mathrm{cm}^{2}$ & $A_{\text {LProdMax }}$ & 65000 \\
\hline LDTreeDev & Allometric parameter of length-diameter relationship of branches, LDT=LDB+LDRatioDev & - & $L D_{\mathrm{T}}$ & $-0.8 / 0.8$ \\
\hline $\operatorname{BetaD}(\operatorname{Min} / \operatorname{Max})$ & Maximum relative increase in height growth when $I_{\mathrm{M}}<I_{\mathrm{T}}$ & - & $\beta_{\mathrm{D}}$ & $0.1 / 0.3$ \\
\hline LightThreshApical & Light intensity threshold regulating apical dominance of trunk apical meristem & $\mu \mathrm{mol} \mathrm{m} \mathrm{m}^{-2}$ & $I_{\mathrm{T}}$ & $30 / 100$ \\
\hline BetaS & Shape parameter regulating apical dominance of trunk apical meristem & - & $\beta_{\mathrm{S}}$ & 3 \\
\hline CarbonOverheadCosts & Carbon overhead costs & - & $C_{0}$ & 1.45 \\
\hline CBLratio & $\mathrm{C}$-mass to biomass ratio of leaves & $\mathrm{gC} \mathrm{g}^{-1}$ & $C B L_{\text {ratio }}$ & 0.5 \\
\hline CBWratio & C-mass to biomass ratio of wood & $\mathrm{gC} \mathrm{g}^{-1}$ & $C B W_{\text {ratio }}$ & 0.5 \\
\hline MortalityDisturbanceRate & Average relative mortality rate in a disturbance event (if $\operatorname{TrMortDist}=1$ ) & $a^{-1}$ & $m_{\text {Dist }}$ & 0 \\
\hline MortalityDisturbanceFrequency & Frequency of disturbances (average number of years between two events) & a & $F_{\text {Dist }}$ & 0 \\
\hline AngleFirstOrderSideView (Min/Max) & Angle of first order branches from side view & $\circ$ & $\alpha_{\mathrm{SFO}}$ & $0 / 40$ \\
\hline PhyllotaxisFirstOrder (Min/Max) & Number of first order branches arranged in a $360^{\circ}$ circle & - & $P h_{\mathrm{FO}}$ & $3 / 5$ \\
\hline AngleSecondOrderTopView (Min/Max) & Angle between second and first order branch from top view & $\circ$ & $\alpha_{\mathrm{TSO}}$ & $20 / 60$ \\
\hline $\operatorname{Imax}$ & Light intensity above canopy & $\mu \mathrm{mol} \mathrm{m} \mathrm{m}^{-2} \mathrm{~s}^{-1}$ & $I_{\max }$ & 900 \\
\hline InitialDiamter & Initial diameter of seedling (fixed value) & $\mathrm{m}$ & $D_{\text {ini }}$ & 0.0005 \\
\hline InternodeLengthBranchMin (Min/Max) & Species-specific minimum branch internode length & $\mathrm{m}$ & $L_{\text {IBMin }}$ & $0.3 / 0.4$ \\
\hline InternodeLengthTrunkMin (Min/Max) & Species-specific minimum trunk internode length & $\mathrm{m}$ & $L_{\text {ITMin }}$ & $0.3 / 0.5$ \\
\hline InternodeLengthTrunkMax (Min/Max) & Species-specific maximum trunk internode length & $\mathrm{m}$ & $L_{\text {ITMax }}$ & $0.5 / 0.7$ \\
\hline KInt (Min/Max) & Factor controlling the relationship between internode length and total annual length growth & - & $k_{\text {int }}$ & $0.01 / 0.02$ \\
\hline LightExtinctionCoeff & Light extinction coefficient (Lambert-Beer equation) & - & $k_{\mathrm{L}}$ & 0.6 \\
\hline LDBranch & Allometric parameter of length-diameter relationship of branches & - & $L D_{\mathrm{B}}$ & 3 \\
\hline LPratio & Ratio between leaf area an pipe cross-sectional area & $\mathrm{cm}^{2} \mathrm{~cm}^{-2}$ & $L P_{\text {ratio }}$ & 40000 \\
\hline DistanceVoxelLightCal & Maximal distance of surrounding voxels to be considered in light calculation & $\mathrm{m}$ & $L R$ & 4 \\
\hline MinLeafBiomass & Minimum leaf biomass below which leaf compartment is removed & g & $B_{\mathrm{LMin}}$ & 30 \\
\hline MortalityBiomassRate & Parameter of biomass-based tree mortality rate & $\mathrm{g}^{-1} \mathrm{a}^{-1}$ & $m_{T B}$ & 0.032 \\
\hline MortalityBiomassScalingExponent & Scaling exponent in biomass-based tree mortality rate & - & $M_{T S}$ & 0.13 \\
\hline MortalityNeighMinDiameter & Trees with a diameter $>D_{\mathrm{NMin}}$ can create gaps (if TrMortNeight $=1$ ) & $\mathrm{cm}$ & $D_{\mathrm{NMin}}$ & 0.15 \\
\hline MortalityNeighRate & Trees affected by falling trees die with a probability of $m_{\text {Neigh }}$ & $\mathrm{a}^{-1}$ & $m_{\text {Neigh }}$ & 0.05 \\
\hline RespirationRateWood & Respiration rate per gram of sapwood & $\mathrm{gC} \mathrm{g}^{-1} \mathrm{~d}^{-1}$ & $R_{\mathrm{w}}$ & 0.0005 \\
\hline NumberSeedlingPerHa (Min/Max) & Number of seedlings dispersed at each time step (per hectare) & $h a^{-1} a^{-1}$ & $n_{\text {Seed }}$ & $500 / 500$ \\
\hline
\end{tabular}


StochasticityTwisting (Min/Max)

Parameter specifying whether stochastic variations of structural traits are simulated

(Stochasticity $=1$ ) or not (Stochasticity $=0$ )

Maximal rotation along the main growth axis (if Stochasticity $=1$ )

StochasticityAngleSecondOrderTopView (Min/Max) Maximum deviation from $\alpha_{\mathrm{TSO}}$ (if Stochasticity $=1$ )

StochasticityTropismStrength (Min/Max)

Maximum deviation from $S_{\text {Top }}$ (if Stochasticity $=1$ )

StochasticityAngleFirstOrderSideView (Min/Max)

StochasticityAngleFirstOrderTopView (Min/Max)

StopCriterionBasalArea

TropismStrength (Min/Max)

WoodDensity (Min/Max)

PipeReuseFactor (Min/Max)

Tyear

Hsun

TreeCompetionNum

TreeCompetionDist

BranchMortMethod

BranchMortRandomRate

BranchMortMassRate

BranchMortMassScalingExponent

TreeMortNeigh

Maximum deviation from $\alpha_{\mathrm{SFO}}$ (if Stochasticity $=1$ )

Maximum deviation from $\alpha_{\text {TFO }}$ (if Stochasticity $=1$ )

Model stops and continues with next replicate if the total basal area exceeds $B A_{\text {stop }}$

Strength of tropism (negative values: phototropism; positive: gravitropism)

Wood density

Pipe-reuse factor

Number of days per year suitable for photosynthesis

Assumed number of sun hours per day

Number of additional trees competing with tree (only if SimualteForest $=0$ )

Distance of additional competing trees from tree (only if SimualteForest $=0$ )

Parameter specifying whether branches are removed only if the lost all leaf compartment

(BrMortMethod $=0$ ), or if they are additionally removed randomly (BrMortMethod $=1$ ) or base on their biomass (BrMortMethod $=2$ )

Random branch mortality rate (if BrMortMethod=1)

Parameter of biomass-based branch mortality rate (if BrMortMethod $=2$ )

Scaling exponent in biomass-based branch mortality rate (if BrMortMethod=2)

Parameter specifying if tree mortality due to falling neighboring trees is simulated

$($ TrMortNeight $=1)$ or not $($ TrMortNeight $=0)$

TreeMortCarbon

TreeMortDist

PipeLengthMethod

FormFactorWood

SafetyFactorTrunk

EdgeC

BrCollide

Parameter specifying if tree mortality due to carbon starvation is simulated (TrMortDist=1) or not $($ TrMortDist $=0)$

Parameter specifying if tree mortality due to disturbances is simulated (TrMortDist=1) or not (TrMortDist $=0$ )

Parameter specifying whether pipe length is calculated based on within-tree position

(PipeLengthMethod $=1$ ) or based on height only (PipeLengthMethod $=0$ )

Form factor used to calculate trunk biomass

Safety factor for trunk growth.

Parameter specifying whether a forest fragment with a real edge $(E d g e C=1)$ or a forest patch within a forest matrix $(\mathrm{EdgeC}=0)$ is simulated

Parameter specifying whether branches stop to grow in length if the collide with surrounding

TrMortCarbon

TrMortDist 
Table C.5. Forest attributes in Neotropical forests based on a literature review. We concentrated on studies covering multiple forest plots or larger forest areas. When available, the number of 1 ha plots or the total study area is given in column 'Extent'. If forest attributes were not estimated based on inventory data, this is mentioned in 'Annotation'. Where available, means \pm sd are shown in column 'Values'. Otherwise, means were estimated, for instance based on published maps. Values in brackets represent the range (min/max) of the forest attributes in the specific study, whereby extreme outliers were removed.

\begin{tabular}{|c|c|c|c|c|c|}
\hline Forest attribute & Unit & Value & Extent & Reference & Annotations \\
\hline Above-ground biomass & $\mathrm{Mg} \mathrm{ha}^{-1}$ & $\sim 280$ & - & (Mitchard et al. 2014) & Amazonia, Remote sensing \\
\hline Above-ground biomass & $\mathrm{Mg} \mathrm{ha}^{-1}$ & $\sim 330$ & - & Mitchard et al. 2014 & Guiana Shield, Remote sensing \\
\hline Above-ground biomass & $\mathrm{Mg} \mathrm{ha}^{-1}$ & $\sim 270$ & - & Mitchard et al. 2014 & SW Amazonia, Remote sensing \\
\hline Above-ground biomass & $\mathrm{Mg} \mathrm{ha}^{-1}$ & $\sim 280(200 / 400)$ & $\mathrm{n}=82$ & Malhi et al. 2015 & Amazonia \\
\hline Above-ground biomass & $\mathrm{Mg} \mathrm{ha}^{-1}$ & 253 & $\mathrm{n}=28$ & Banin et al. 2014 & Amazonia \\
\hline Above-ground biomass & $\mathrm{Mg} \mathrm{ha}^{-1}$ & $195(108 / 308)$ & $\mathrm{n}=35$ & Feldpausch et al. 2012 & Brazilian Shield \\
\hline Above-ground biomass & $\mathrm{Mg} \mathrm{ha}^{-1}$ & $344(237 / 510)$ & $\mathrm{n}=44$ & Feldpausch et al. 2012 & Eastern-Central Amazonia \\
\hline Above-ground biomass & $\mathrm{Mg} \mathrm{ha}^{-1}$ & $434(291 / 728)$ & $\mathrm{n}=45$ & Feldpausch et al. 2012 & Guyana Shield \\
\hline Above-ground biomass & $\mathrm{Mg} \mathrm{ha}^{-1}$ & $252(142 / 392)$ & $\mathrm{n}=101$ & Feldpausch et al. 2012 & Western Amazonia \\
\hline Above-ground biomass & $\mathrm{Mg} \mathrm{ha}^{-1}$ & $\sim 276$ & $\mathrm{n}=20$ & Baker et al. 2004 & NW Amazonia \\
\hline Above-ground biomass & $\mathrm{Mg} \mathrm{ha}^{-1}$ & $\sim 340$ & $\mathrm{n}=17$ & Baker et al. 2004 & Eastern-Central Amazonia \\
\hline Above-ground biomass & $\mathrm{Mg} \mathrm{ha}^{-1}$ & $\sim 246$ & $\mathrm{n}=19$ & Baker et al. 2004 & SW Amazonia \\
\hline Above-ground biomass & $\mathrm{Mg} \mathrm{ha}^{-1}$ & $\sim 300(250 / 350)$ & $\mathrm{n}=227$ & Malhi et al. 2006 & Amazon-wide interpolation \\
\hline Above-ground biomass & $\mathrm{Mg} \mathrm{ha}^{-1}$ & $287.8 \pm 105.0$ & $\mathrm{n}=33$ & Slik et al. 2013 & Amazonia \\
\hline AGB residence time & a & $\sim 40(20 / 100)$ & $\mathrm{n}=82$ & Malhi et al. 2015 & Amazonia \\
\hline AGB residence time & a & $\sim 50$ & $\mathrm{n}=127$ & Galbraith et al. 2013 & Neotropics \\
\hline AGB residence time & a & $\sim 50$ & - & Malhi et al. 2011 & Amazonia \\
\hline AGB residence time & $\mathrm{a}$ & $\sim 52$ & - & Malhi et al. 2013 & W Amazonia \\
\hline AGB residence time & $\mathrm{a}$ & $\sim 80$ & - & Malhi et al. 2013 & E Amazonia \\
\hline Basal area & $\mathrm{m}^{2} \mathrm{ha}^{-1}$ & $22.2 \pm 5.3(7.1 / 32.4)$ & $\mathrm{n}=35$ & Feldpausch et al. 2011 & Brazilian Shield \\
\hline Basal area & $\mathrm{m}^{2} \mathrm{ha}^{-1}$ & $23.5 \pm 10.2(1.7 / 47.7)$ & $\mathrm{n}=44$ & Feldpausch et al. 2011 & Eastern-Central Amazonia \\
\hline Basal area & $\mathrm{m}^{2} \mathrm{ha}^{-1}$ & $27.6 \pm 5.4(16 / 37)$ & $\mathrm{n}=45$ & Feldpausch et al. 2011 & Guyana Shield \\
\hline Basal area & $\mathrm{m}^{2} \mathrm{ha}^{-1}$ & $27.8 \pm 2.9(15.6 / 39)$ & $\mathrm{n}=101$ & Feldpausch et al. 2011 & Western Amazonia \\
\hline Basal area & $\mathrm{m}^{2} \mathrm{ha}^{-1}$ & $28.2(21.7 / 36.8)$ & $\mathrm{n}=50$ & Lewis et al. 2004a & Amazonia \\
\hline Basal area & $\mathrm{m}^{2} \mathrm{ha}^{-1}$ & $\sim 26(20 / 32)$ & - & Mitchard et al. 2014 & Amazonia, Remote sensing \\
\hline Basal area & $\mathrm{m}^{2} \mathrm{ha}^{-1}$ & 28.1 & $\mathrm{n}=28$ & Banin et al. 2014 & Amazonia \\
\hline Basal area & $\mathrm{m}^{2} \mathrm{ha}^{-1}$ & $28.1 \pm 1.6$ & $\mathrm{n}=15$ & Chao et al. 2008 & North-Western Amazonia \\
\hline
\end{tabular}




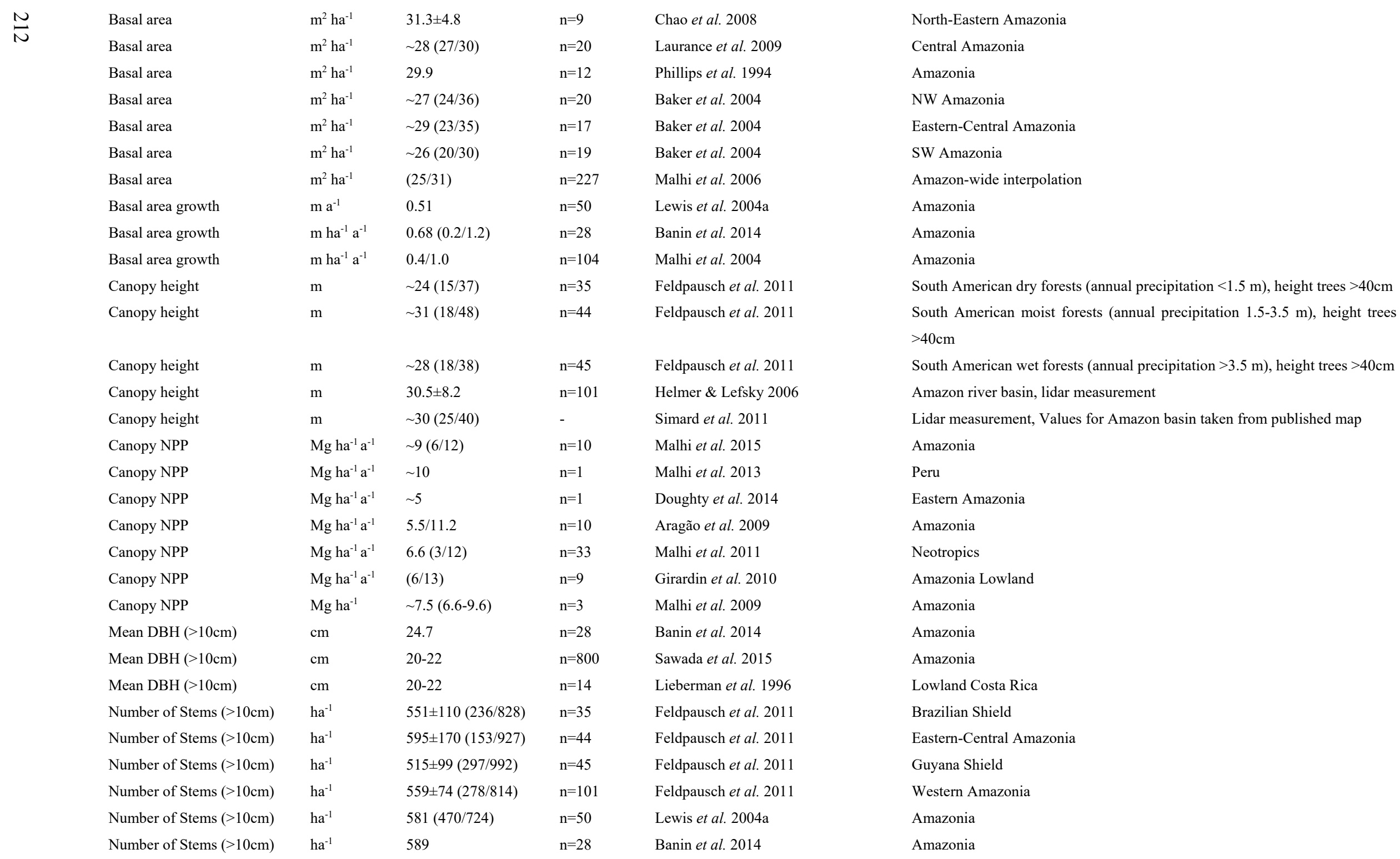




\begin{tabular}{|c|c|c|c|c|c|}
\hline Number of Stems $(>10 \mathrm{~cm})$ & $\mathrm{ha}^{-1}$ & $595 \pm 23$ & $\mathrm{n}=15$ & Chao et al. 2008 & North-Western Amazonia \\
\hline Number of Stems $(>10 \mathrm{~cm})$ & $\mathrm{ha}^{-1}$ & $560 \pm 35$ & $\mathrm{n}=9$ & Chao et al. 2008 & North-Eastern Amazonia \\
\hline Number of Stems $(>10 \mathrm{~cm})$ & $\mathrm{ha}^{-1}$ & $645(500 / 750)$ & $\mathrm{n}=12$ & Phillips et al. 1994 & Amazonia \\
\hline Number of Stems $(>1 \mathrm{~cm})$ & $\mathrm{ha}^{-1}$ & $2600 / 4700$ & 50 ha & Chave et al. 2003 & Barro Colorado Island \\
\hline Number of Stems $(>1 \mathrm{~cm})$ & $\mathrm{ha}^{-1}$ & $2000 / 2300$ & $\mathrm{n}=8$ & DeWalt \& Chave 2004 & Neotropical lowland \\
\hline Number of Stems $(>1 \mathrm{~cm})$ & $\mathrm{ha}^{-1}$ & $\sim 4000$ & $\mathrm{n}=3$ & Muller-Landau et al. 2006b & Neotropical lowland \\
\hline Stem turnover $(>10 \mathrm{~cm})$ & $a^{-1}$ & $\sim 2(0.5 / 4)$ & $\mathrm{n}=97$ & Phillips et al. 2004b & Pan-Amazon study \\
\hline Stem turnover $(>10 \mathrm{~cm})$ & $a^{-1}$ & $1.5 \pm(0.5 / 3.5)$ & $\mathrm{n}=67$ & Phillips 1996 & Amazonia, Mean tree turnover increased from $\sim 1 \%$ to $2 \%$ from $1956-1991$ \\
\hline Stem turnover $(>10 \mathrm{~cm})$ & $a^{-1}$ & $\sim 1.8(1.2 / 3.1)$ & $\mathrm{n}=50$ & Lewis et al. 2004a & Amazonia \\
\hline Stem turnover $(>10 \mathrm{~cm})$ & $a^{-1}$ & $2.34 \pm 0.31$ & $\mathrm{n}=15$ & Chao et al. 2008 & North-Western Amazonia, mortality rate \\
\hline Stem turnover $(>10 \mathrm{~cm})$ & $a^{-1}$ & $1.21 \pm 0.53$ & $\mathrm{n}=9$ & Chao et al. 2008 & North-Eastern Amazonia, mortality rate \\
\hline Stem turnover $(>10 \mathrm{~cm})$ & $a^{-1}$ & $(1 / 1.7)$ & $\mathrm{n}=20$ & Laurance et al. 2009 & Central Amazonia \\
\hline Stem turnover $(>10 \mathrm{~cm})$ & $a^{-1}$ & $\sim 1.85(1.5 / 2.5)$ & $\mathrm{n}=12$ & Phillips et al. 1994 & Amazonia \\
\hline Stem turnover $(>10 \mathrm{~cm})$ & $a^{-1}$ & $(1 / 2)$ & $n=14$ & Lewis et al. 2011 & Amazonia \\
\hline Stem turnover $(>10 \mathrm{~cm})$ & $a^{-1}$ & $(1.5 / 2.5) 95 \%,(1-5)$ & $\mathrm{n}=50$ & Phillips et al. 2009 & Amazonia \\
\hline Total ANPP & $\mathrm{Mg} \mathrm{ha}^{-1} \mathrm{a}^{-1}$ & $\sim 16$ & 2 ha & Doughty et al. 2014 & Terra preta \\
\hline Total ANPP & $\mathrm{Mg} \mathrm{ha}^{-1} \mathrm{a}^{-1}$ & $\sim 14.5$ & $n=3$ & Malhi et al. 2009 & Amazonia \\
\hline Total ANPP & $\mathrm{Mg} \mathrm{ha}^{-1} \mathrm{a}^{-1}$ & $10 / 22$ & $\mathrm{n}=10$ & Aragão et al. 2009 & Amazonia \\
\hline Total ANPP & $\operatorname{Mg~ha}^{-1} \mathrm{a}^{-1}$ & $10 / 20$ & $\mathrm{n}=9$ & Girardin et al. 2010 & Amazonia Lowland \\
\hline Total ANPP & $\mathrm{Mg} \mathrm{ha}^{-1} \mathrm{a}^{-1}$ & $\sim 12.9$ & 21 ha & Chambers et al. 2001 & Amazonia Lowland \\
\hline Leaf area index & $\mathrm{m} \mathrm{m}^{-2}$ & $\sim 4.7$ & - & Asner et al. 2004 & Amazonia (mean) \\
\hline Leaf area index & $\mathrm{m} \mathrm{m}^{-2}$ & $\sim 4.8$ & - & Caldararu et al. 2012 & Central and Southern Amazonia; Satellite Observations \\
\hline Leaf area index & $\mathrm{m} \mathrm{m}^{-2}$ & $\sim 4.0$ & - & Caldararu et al. 2012 & Eastern Amazonia; Satellite Observations \\
\hline Leaf area index & $\mathrm{m} \mathrm{m}^{-2}$ & $\sim 4.7$ & - & Myneni et al. 2007 & Amazonia (mean) \\
\hline
\end{tabular}


Table C.6. Value ranges of model parameters. Many parameters used in the present model have natural ranges, which were estimated based on literature values.

\begin{tabular}{|c|c|c|c|c|}
\hline Symbol & Unit & Explanation & Value (range) & References \\
\hline$A_{\text {LMax }}$ & $\mathrm{cm}^{2}$ & Maximum leaf area in voxel & $10000-20000$ & Model specific parameter, own estimates \\
\hline$A_{\text {LProdMax }}$ & $\mathrm{cm}^{2}$ & Absolute maximum leaf area production per leaf compartment & $20000-40000$ & Model specific parameter, own estimates \\
\hline$L D_{\mathrm{T}}$ & - & $\begin{array}{l}\text { Allometric parameter of length-diameter relationship of branches, } \\
\text { LDT=LDB+LDRatioDev }\end{array}$ & $-1-1$ & $\begin{array}{l}\text { McMahon 1971; Bertram 1989; Niklas 1995; West, Brown \& Enquist 1999; van } \\
\text { Gelder, Poorter \& Sterck 2006; Banin et al. } 2012\end{array}$ \\
\hline$C_{0}$ & - & Carbon overhead costs (construction costs) & $1.4-1.5$ & $\begin{array}{l}\text { Poorter \& De Jong 1999; Cannell \& Thornley 2000; Sterck et al. 2005; Pons \& } \\
\text { Poorter } 2014\end{array}$ \\
\hline$C B L_{\text {ratio }}$ & $\mathrm{gC} \mathrm{g}^{-1}$ & C-mass to biomass ratio of leaves (carbon content) & $0.45-0-5$ & Houghton et al. 2001; Elias \& Potvin 2003; Martin \& Thomas 2011 \\
\hline$C B W_{\text {ratio }}$ & $\mathrm{gC} \mathrm{g}^{-1}$ & C-mass to biomass ratio of wood (carbon content) & $0.45-0.5$ & Houghton et al. 2001; Elias \& Potvin 2003; Martin \& Thomas 2011 \\
\hline$I_{\max }$ & $\mu \mathrm{mol} \mathrm{m} \mathrm{m}^{-2} \mathrm{~s}^{-1}$ & Light intensity above canopy & $500-1200$ & $\begin{array}{l}\text { Chazdon \& Fetcher 1984; Berry, Varney \& Flanagan 1997; Valladares, Allen \& } \\
\text { Pearcy 1997; Sterck et al. 2011; Seyoum et al. } 2014\end{array}$ \\
\hline$k_{\mathrm{L}}$ & - & Light extinction coefficient (Lambert-Beer equation) & $0.5-0.8$ & Huth \& Ditzer 2000; Kitajima, Mulkey \& Wright 2005b; Malhi et al. 2013 \\
\hline$L D_{\mathrm{B}}$ & - & $\begin{array}{l}\text { Allometric parameter of length-diameter relationship of branches. } \\
\text { Important: } 1.2-3 \text { for trunks }\end{array}$ & $1.2-3$ & $\begin{array}{l}\text { McMahon 1971; Bertram 1989; Niklas 1995; West, Brown \& Enquist 1999; van } \\
\text { Gelder, Poorter \& Sterck 2006; Banin et al. } 2012\end{array}$ \\
\hline$L P_{\text {ratio }}$ & $\mathrm{cm}^{2} \mathrm{~cm}^{-2}$ & $\begin{array}{l}\text { Ratio between leaf area and pipe cross-sectional area. Important: } \\
\text { PipeReuseFactor is important here }\end{array}$ & $3000-20000$ & Wright et al. 2006; Calvo-Alvarado, McDowell \& Waring 2008; Patiño et al. 2012 \\
\hline$m_{T B}$ & $g^{-1} a^{-1}$ & Parameter of biomass-based tree mortality rate & $0.01-0.05$ & Model specific parameter, own estimates \\
\hline$M_{T S}$ & - & $\begin{array}{l}\text { Scaling exponent in biomass-based tree mortality rate, The metabolic } \\
\text { theory predicts a scaling exponent of } 1 / 4 \text {, but significant deviations } \\
\text { have been observed. }\end{array}$ & $0.1-0.4$ & Brown et al. 2004; Muller-Landau et al. 2006a; b \\
\hline$D_{\mathrm{NMin}}$ & $\mathrm{cm}$ & Trees with a diameter $>D_{\mathrm{NMin}}$ can create gaps (if TrMortNeight $=1$ ) & $0.1-0.3$ & Model specific parameter, own estimates \\
\hline$m_{\text {Neigh }}$ & $a^{-1}$ & Trees affected by falling trees die with a probability of $m_{\text {Neigh }}$ & $0.05-0.3$ & Model specific parameter, own estimates \\
\hline$R_{\mathrm{w}}$ & $\mathrm{gC} \mathrm{g}^{-1} \mathrm{~d}^{-1}$ & Respiration rate per gram of sapwood & $10^{-4}-10^{-6}$ & $\begin{array}{l}\text { Penning De Vries 1975; Ryan et al. 1994, 1995; Vose \& Ryan 2002; Sterck et al. } \\
2005\end{array}$ \\
\hline$n_{\text {Seed }}$ & $h a^{-1} a^{-1}$ & Number of seedlings dispersed at each time step (per hectare) & $100-500$ & Model specific parameter, own estimates \\
\hline$S_{\mathrm{F}}$ & - & Factor regulating the shortening of branches with their order & $0.7-0.9$ & Bertram 1989; Perttunen et al. 1996; Perttunen, Sieva \& Nikinmaa 1998 \\
\hline$S I$ & - & Site index describing the relative quality of the forest patch & $0.5-1$ & Model specific parameter, own estimates \\
\hline SLA & $\mathrm{cm}^{2} \mathrm{~g}^{-1}$ & Specific leaf area, mean value for Amazonia $\sim 90-110$ & $50-200$ & $\begin{array}{l}\text { Poorter 1999; Rijkers et al. 2000; Kitajima, Mulkey \& Wright 2005a; Wright et al. } \\
\text { 2005, 2010; Rozendaal et al. 2006; Markesteijn et al. 2007; Domingues, Martinelli } \\
\text { \& Ehleringer 2007; Poorter et al. 2009; Malhi et al. 2009; Patiño et al. 2012; } \\
\text { Niinemets, Keenan \& Hallik } 2015\end{array}$ \\
\hline$\rho_{\mathrm{W}}$ & $\mathrm{g} \mathrm{cm}^{-3}$ & Wood density, mean value 0.63 for Amazonia & $0.4-0.8$ & Baker et al. 2004; Patiño et al. 2009; Quesada et al. 2012; Iida et al. 2014 \\
\hline$P_{R U}$ & - & Pipe-reuse factor & $0.5-0.9$ & Mäkelä 1986, 2002; Perttunen et al. 1996; Sterck \& Schieving 2007 \\
\hline$t_{\text {year }}$ & d & Number of days per year suitable for photosynthesis & $180-360$ & Model specific parameter, own estimates \\
\hline$h_{\text {sun }}$ & $\mathrm{h}$ & Assumed number of sun hours per day & 6-10 & Model specific parameter, own estimates \\
\hline$S T$ & - & Safety factor for trunk growth. & $0.5-0.9$ & Sterck \& Bongers 1998; van Gelder et al. 2006; Osunkoya et al. 2007 \\
\hline
\end{tabular}


Table C.7. Results of sensitivity analysis, in which the effect of changes in important model parameters on the main forest attributes were recorded. The parameters of the best model (Table C.4) form the basis of this analysis, and single parameters were varied over the range shown in the column 'Value range'. The arrow indicate whether increasing parameters values had a strong positive effect $(\uparrow)$, a slight positive effect $(\nearrow)$, no or an indifferent effect $(\rightarrow)$, strong negative effect $(\downarrow)$ or a slight negative effect $(\searrow)$ on the forest attributes.

\begin{tabular}{|c|c|c|c|c|c|c|c|c|c|c|c|c|c|c|}
\hline Symbol & Unit & Value range & $\begin{array}{c}\text { Stem } \\
\text { density } \\
(>10 \mathrm{~cm})\end{array}$ & $\begin{array}{c}\text { Stem } \\
\text { density }\end{array}$ & $\begin{array}{l}\text { Basal } \\
\text { area }\end{array}$ & AGB & $\begin{array}{c}\text { Canopy } \\
\text { height }\end{array}$ & $\begin{array}{c}\text { Mean } \\
\text { DBH } \\
(>10 \mathrm{~cm})\end{array}$ & LAI & $\begin{array}{l}\text { Total } \\
\text { ANPP }\end{array}$ & $\begin{array}{c}\text { Canopy } \\
\text { NPP }\end{array}$ & $\begin{array}{c}\text { Basal } \\
\text { area } \\
\text { growth }\end{array}$ & $\begin{array}{c}\text { Residence } \\
\text { time } \\
\text { AGB }\end{array}$ & $\begin{array}{c}\text { Stem } \\
\text { Turnover } \\
(>10 \mathrm{~cm})\end{array}$ \\
\hline$A_{\text {LMax }}$ & $\mathrm{cm}^{2}$ & $10000-20000$ & $\rightarrow$ & $\rightarrow$ & $\rightarrow$ & $\rightarrow$ & $\rightarrow$ & $\rightarrow$ & $\rightarrow$ & $\rightarrow$ & $\rightarrow$ & $\rightarrow$ & $\rightarrow$ & $\rightarrow$ \\
\hline$A_{\text {LProdMax }}$ & $\mathrm{cm}^{2}$ & $20000-80000$ & $\searrow$ & $\downarrow$ & $\nearrow$ & $\uparrow$ & $\searrow$ & $\uparrow$ & $\lambda$ & $\lambda$ & $\lambda$ & $\lambda$ & $\lambda$ & $\rightarrow$ \\
\hline$L D_{\mathrm{T}}$ & - & $-0.5-0.5$ & $\searrow$ & $\rightarrow$ & $\searrow$ & $\rightarrow$ & $\lambda$ & $\rightarrow$ & $\rightarrow$ & $\rightarrow$ & $\rightarrow$ & $\rightarrow$ & $\rightarrow$ & $\rightarrow$ \\
\hline$C_{0}$ & - & $1.4-1.6$ & $\rightarrow$ & $\rightarrow$ & $\rightarrow$ & $\searrow$ & $\searrow$ & $\rightarrow$ & $\rightarrow$ & $\searrow$ & $\rightarrow$ & $\rightarrow$ & $\rightarrow$ & $\rightarrow$ \\
\hline$C B L_{\text {ratio }}$ & $\mathrm{gC} \mathrm{g}^{-1}$ & $0.4-0.5$ & $\rightarrow$ & $\rightarrow$ & $\rightarrow$ & $\rightarrow$ & $\rightarrow$ & $\rightarrow$ & $\rightarrow$ & $\rightarrow$ & $\rightarrow$ & $\rightarrow$ & $\rightarrow$ & $\rightarrow$ \\
\hline$C B W_{\text {ratio }}$ & $\mathrm{gC} \mathrm{g}^{-1}$ & $0.4-0.5$ & $\lambda$ & $\rightarrow$ & $\nu$ & $\searrow$ & $\searrow$ & $\searrow$ & $\rightarrow$ & $\searrow$ & $\rightarrow$ & $\rightarrow$ & $\nu$ & $\rightarrow$ \\
\hline$I_{\max }$ & $\mu \mathrm{mol} \mathrm{m} \mathrm{m}^{-2} \mathrm{~s}^{-1}$ & $300-1200$ & $\rightarrow$ & $\searrow$ & $\uparrow$ & $\uparrow$ & $\uparrow$ & $\uparrow$ & $\uparrow$ & $\uparrow$ & $\uparrow$ & $\uparrow$ & $\rightarrow$ & $\rightarrow$ \\
\hline$k_{\mathrm{L}}$ & - & $0.5-0.9$ & $\downarrow$ & 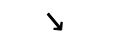 & $\downarrow$ & $\downarrow$ & $\rightarrow$ & $\rightarrow$ & $\downarrow$ & $\downarrow$ & $\downarrow$ & $\downarrow$ & $\rightarrow$ & $\rightarrow$ \\
\hline$L D_{\text {в }}$ & - & $1.5-3.5$ & $\searrow$ & $\searrow$ & $\lambda$ & $\lambda$ & $\lambda$ & $\lambda$ & $\rightarrow$ & $\lambda$ & $\rightarrow$ & $\rightarrow$ & $\rightarrow$ & $\rightarrow$ \\
\hline$L P_{\text {ratio }}$ & $\mathrm{cm}^{2} \mathrm{~cm}^{-2}$ & $10000-60000$ & $\downarrow$ & $\downarrow$ & $\downarrow$ & $\uparrow$ & $\uparrow$ & $\uparrow$ & $\lambda$ & $\rightarrow$ & $\rightarrow$ & $\downarrow$ & $\uparrow$ & $\downarrow$ \\
\hline$m_{T B}$ & $g^{-1} a^{-1}$ & $0.01-0.1$ & $\downarrow$ & $\downarrow$ & $\downarrow$ & $\downarrow$ & $\searrow$ & $\lambda$ & $\rightarrow$ & $\rightarrow$ & $\rightarrow$ & $\rightarrow$ & $\downarrow$ & $\uparrow$ \\
\hline$M_{T S}$ & - & $0.05-0.3$ & $\uparrow$ & $\downarrow$ & $\uparrow$ & $\uparrow$ & $\rightarrow$ & $\rightarrow$ & $\rightarrow$ & $\rightarrow$ & $\rightarrow$ & $\rightarrow$ & $\uparrow$ & $\downarrow$ \\
\hline$D_{\mathrm{NMin}}$ & $\mathrm{cm}$ & $0.1-0.2$ & $\rightarrow$ & $\rightarrow$ & $\rightarrow$ & $\rightarrow$ & $\rightarrow$ & $\rightarrow$ & $\rightarrow$ & $\rightarrow$ & $\rightarrow$ & $\rightarrow$ & $\rightarrow$ & $\rightarrow$ \\
\hline$R_{\mathrm{w}}$ & $\mathrm{gC} \mathrm{g}^{-1} \mathrm{~d}^{-1}$ & $10^{-5}-10^{-4}$ & $\rightarrow$ & $\rightarrow$ & $\rightarrow$ & $\rightarrow$ & $\rightarrow$ & $\rightarrow$ & $\rightarrow$ & $\rightarrow$ & $\rightarrow$ & $\rightarrow$ & $\rightarrow$ & $\rightarrow$ \\
\hline$n_{\text {Seed }}$ & $h^{-1} a^{-1}$ & $100-800$ & $\lambda$ & $\uparrow$ & $\rightarrow$ & $\rightarrow$ & $\rightarrow$ & $\searrow$ & $\rightarrow$ & $\rightarrow$ & $\rightarrow$ & $\lambda$ & $\rightarrow$ & $\rightarrow$ \\
\hline$S_{\mathrm{F}}$ & - & $0.5-0.95$ & $\downarrow$ & $\searrow$ & $\rightarrow$ & $\rightarrow$ & $\searrow$ & $\lambda$ & $\searrow$ & $\rightarrow$ & $\lambda$ & $\rightarrow$ & $\rightarrow$ & $\rightarrow$ \\
\hline$S I$ & - & $0.5-1$ & $\searrow$ & $\checkmark$ & $\uparrow$ & $\uparrow$ & $\uparrow$ & $\uparrow$ & $\uparrow$ & $\uparrow$ & $\uparrow$ & $\uparrow$ & $\lambda$ & $\searrow$ \\
\hline$S L A$ & $\mathrm{~cm}^{2} \mathrm{~g}^{-1}$ & $50-300$ & $\uparrow$ & $\uparrow$ & $\lambda$ & $\searrow$ & $\downarrow$ & $\downarrow$ & $\pi$ & $\uparrow$ & $\uparrow$ & $\uparrow$ & $\downarrow$ & $\uparrow$ \\
\hline$\rho_{\mathrm{w}}$ & $\mathrm{g} \mathrm{cm}^{-3}$ & $0.4-0.8$ & $\uparrow$ & $\uparrow$ & $\downarrow$ & $\downarrow$ & $\downarrow$ & $\downarrow$ & $\rightarrow$ & $\rightarrow$ & $\rightarrow$ & $\searrow$ & $\searrow$ & $\rightarrow$ \\
\hline$P_{R U}$ & - & $0.3-0.9$ & $\uparrow$ & $\uparrow$ & $\lambda$ & $\searrow$ & $\downarrow$ & $\downarrow$ & $\rightarrow$ & $\rightarrow$ & $\rightarrow$ & $\uparrow$ & $\downarrow$ & $\lambda$ \\
\hline$t_{\text {year }}$ & d & $120-360$ & $\uparrow$ & $\searrow$ & $\uparrow$ & $\uparrow$ & $\rightarrow$ & $\rightarrow$ & $\rightarrow$ & $\uparrow$ & $\uparrow$ & $\uparrow$ & $\downarrow$ & $\lambda$ \\
\hline$h_{\text {sun }}$ & h & $6-12$ & $\downarrow$ & $\downarrow$ & $\uparrow$ & $\uparrow$ & $\uparrow$ & $\uparrow$ & $\uparrow$ & $\uparrow$ & $\uparrow$ & $\uparrow$ & $\lambda$ & $\rightarrow$ \\
\hline$S T$ & - & $0.1-0.5$ & $\rightarrow$ & $\rightarrow$ & $\rightarrow$ & $\rightarrow$ & $\rightarrow$ & $\rightarrow$ & $\rightarrow$ & $\rightarrow$ & $\rightarrow$ & $\rightarrow$ & $\rightarrow$ & $\rightarrow$ \\
\hline
\end{tabular}




\section{Supplementary information to chapter 5}
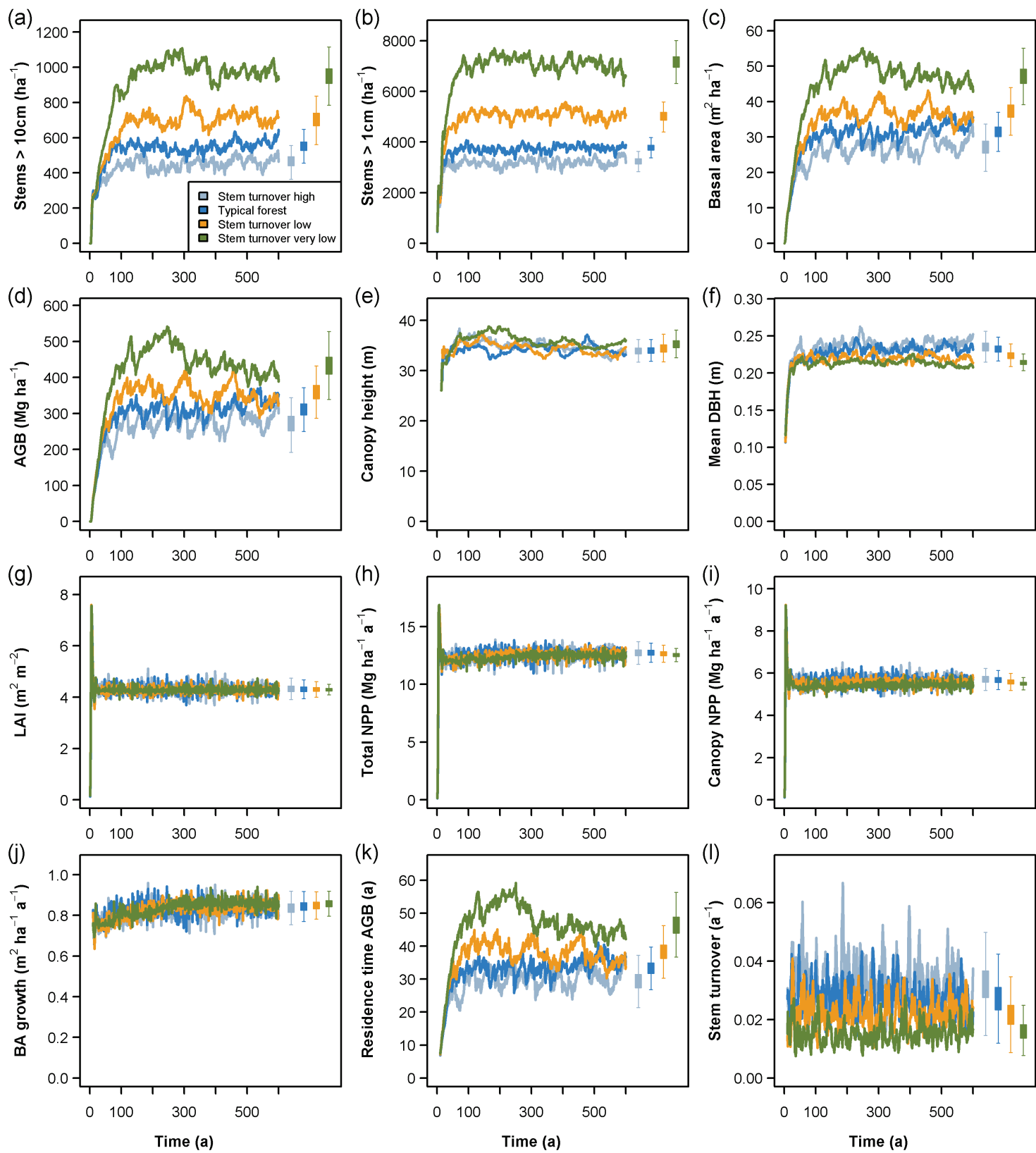

Figure D.1. Long-term dynamics of the simulated forest stands differing in natural forest dynamics used as model scenarios in this study. (a) Stem density of all stems $>10 \mathrm{~cm}$ in DBH, (b) Stem density of all stems $>1 \mathrm{~cm}$ in DBH, (c) Basal area, (d) Above-ground biomass, (e) Canopy height (mean height of all trees $>40 \mathrm{~cm}$ in DBH), (f) Mean diameter of all stems $>10 \mathrm{~cm}$ in DBH, (g) Leaf area index, (h) Total above-ground net primary production, (i) Canopy net primary production (NPP of leaves and $2^{\text {nd }}$ order branches), (j) Basal area growth, (k) Residence time of aboveground biomass, (l) Turnover of all stems $>10 \mathrm{~cm}$ in DBH. 

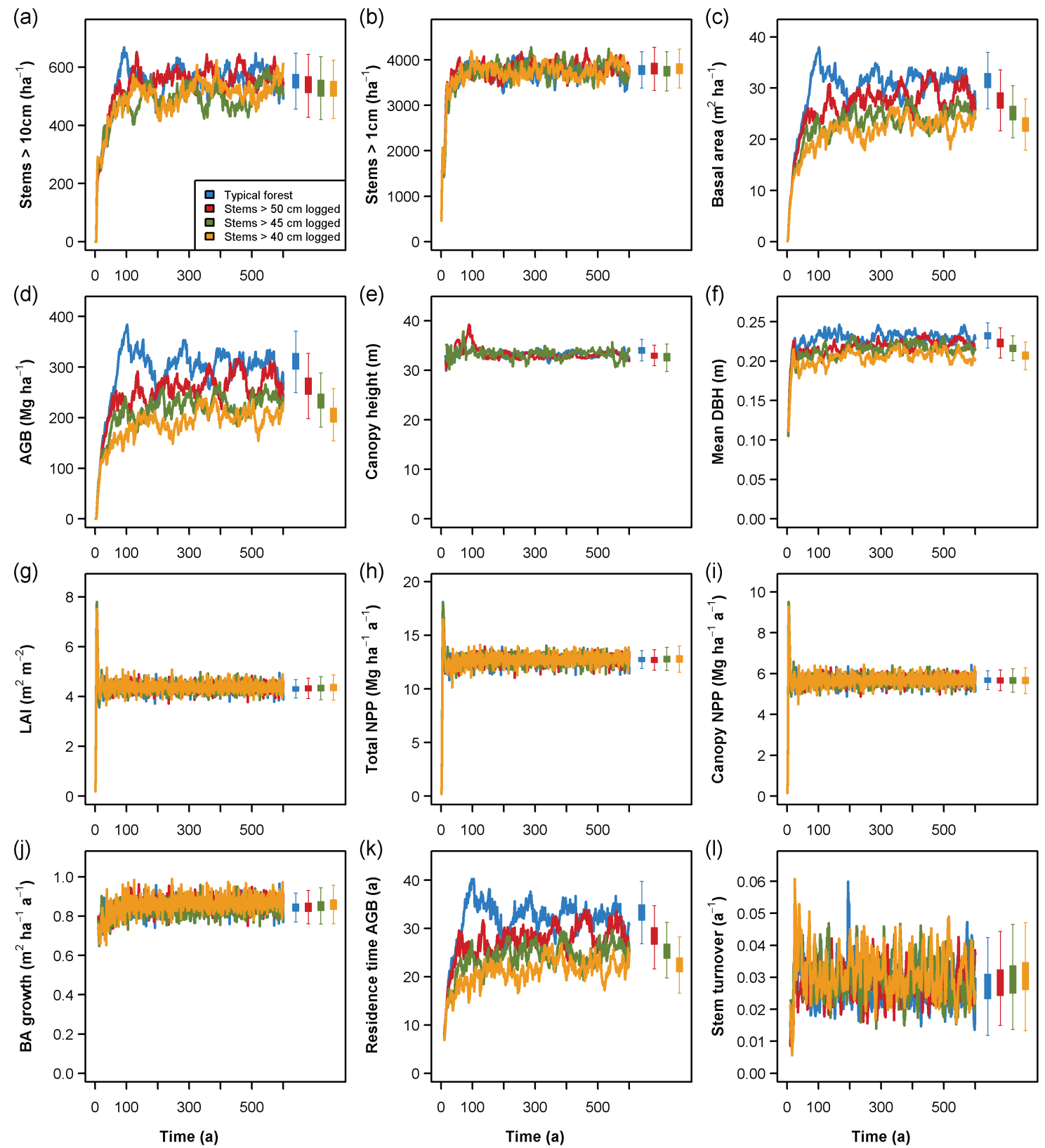

Figure D.2. Long-term dynamics of simulated forest stands differing in logging intensities (a) Stem density of all stems $>10 \mathrm{~cm}$ in DBH, (b) Stem density of all stems $>1 \mathrm{~cm}$ in DBH, (c) Basal area, (d) Above-ground biomass, (e) Canopy height (mean height of all trees $>40 \mathrm{~cm}$ in DBH), (f) Mean diameter of all stems $>10 \mathrm{~cm}$ in DBH, (g) Leaf area index, (h) Total above-ground net primary production, (i) Canopy net primary production (NPP of leaves and $2^{\text {nd }}$ order branches), (j) Basal area growth, (k) Residence time of above-ground biomass, (l) Turnover of all stems $>10 \mathrm{~cm}$ in DBH. 

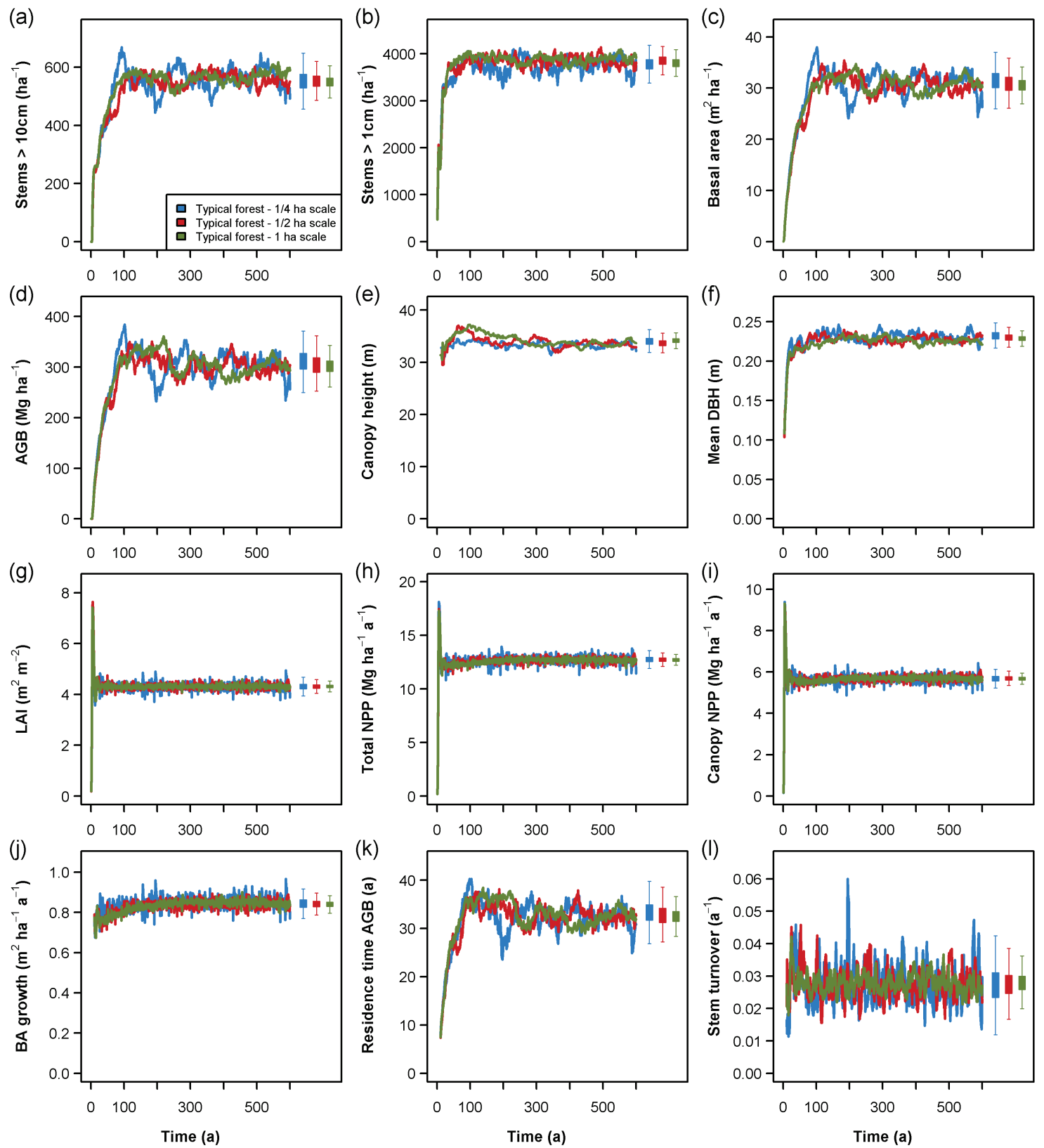

Figure D.3. Long-term dynamics of simulated forest stands differing in fragment size. (a) Stem density of all stems $>$ $10 \mathrm{~cm}$ in DBH, (b) Stem density of all stems $>1 \mathrm{~cm}$ in DBH, (c) Basal area, (d) Above-ground biomass, (e) Canopy height (mean height of all trees $>40 \mathrm{~cm}$ in DBH), (f) Mean diameter of all stems $>10 \mathrm{~cm}$ in DBH, (g) Leaf area index, (h) Total above-ground net primary production, (i) Canopy net primary production (NPP of leaves and $2^{\text {nd }}$ order branches), (j) Basal area growth, (k) Residence time of above-ground biomass, (l) Turnover of all stems $>10 \mathrm{~cm}$ in DBH. 

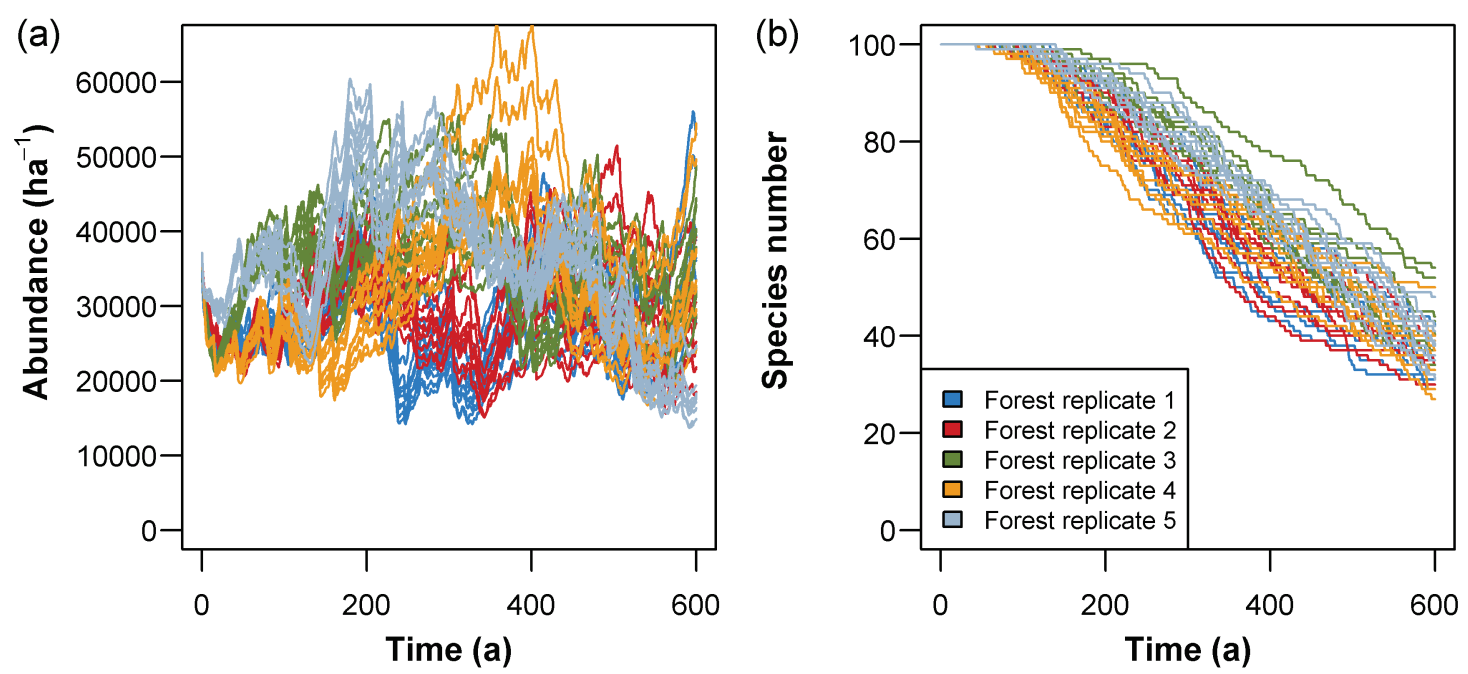

Figure D.4. Simulated long-term dynamics of vascular epiphytes communities. Five replicates of a typical lowland forest stand $(50 \times 50 \mathrm{~m})$ were simulated (see Fig. D. 1 for forest attributes) and used as input data for the epiphyte model. On each of these forest replicates, the development of epiphyte communities which initially consisted of 100 individuals of 100 species was simulated over 600 years and the abundance (a) and species numbers (b) are shown here. Ten different initial species pools were simulated on each forest replicate and their development is shown here.
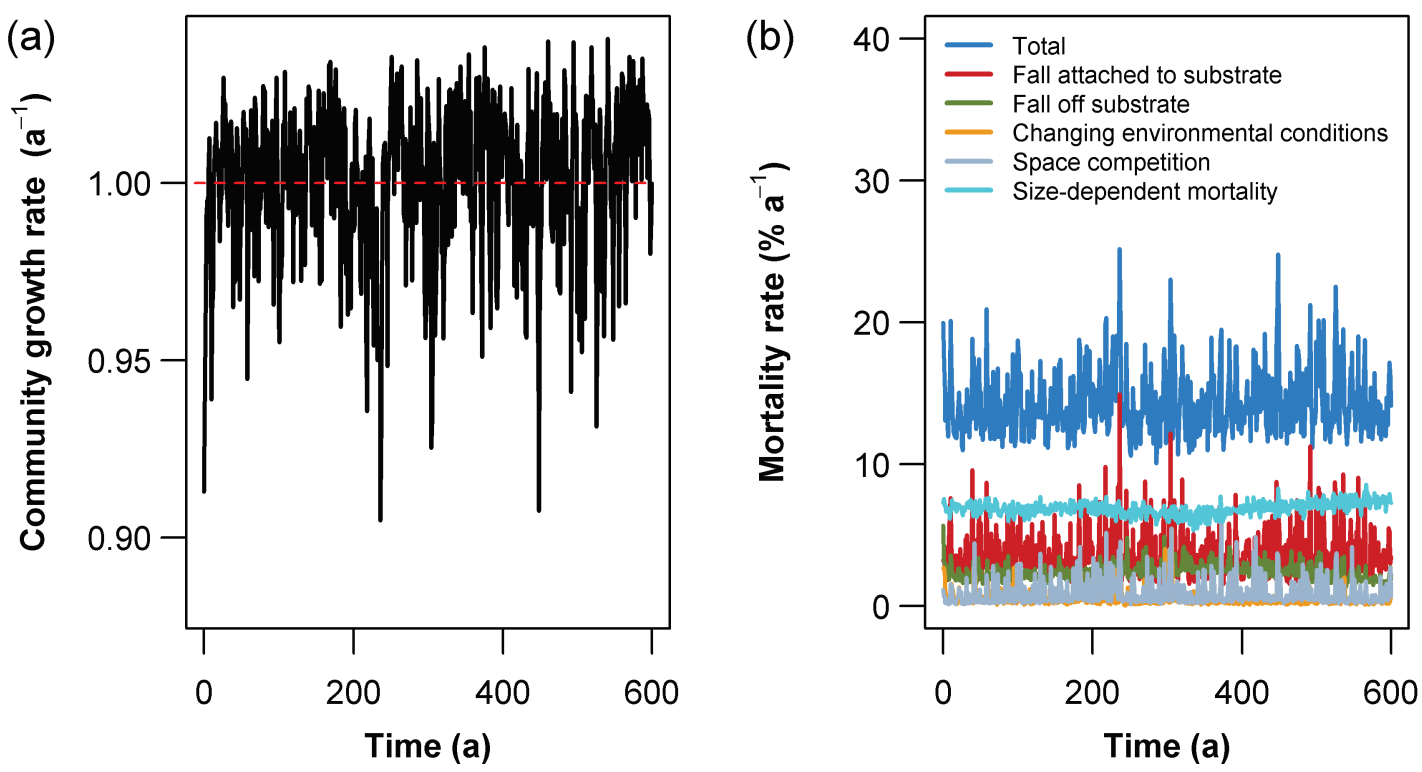

Figure D.5. Community growth rates and partitioning of mortality rates. 


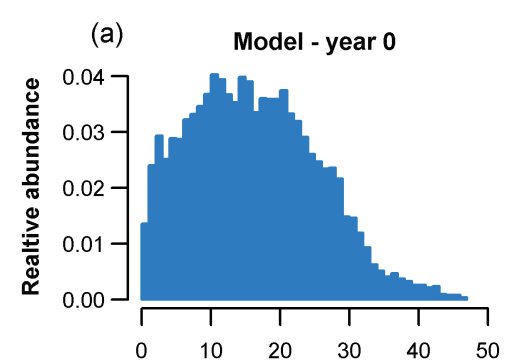

(d)

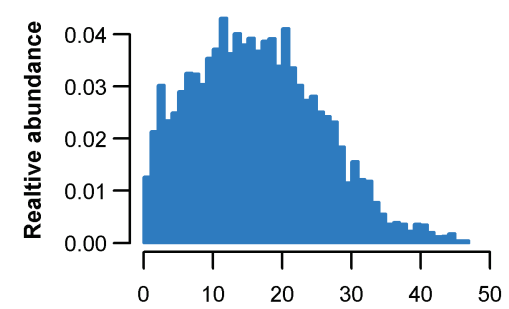

(g)

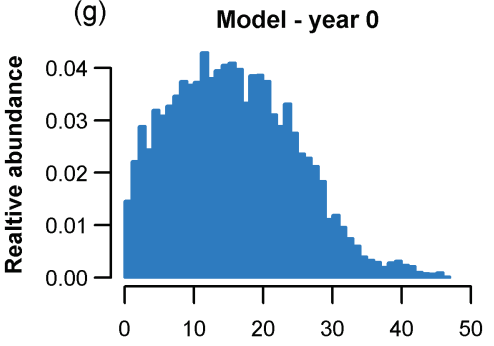

(j) Model - substrate area

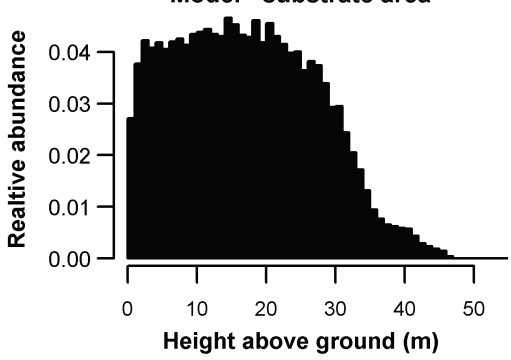

(b)

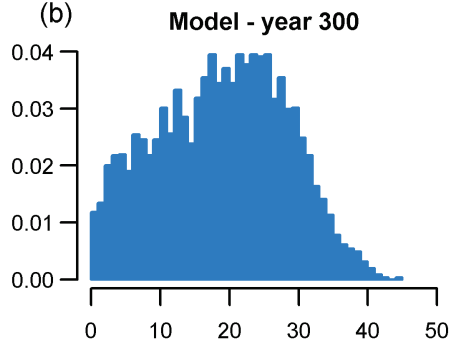

(e)

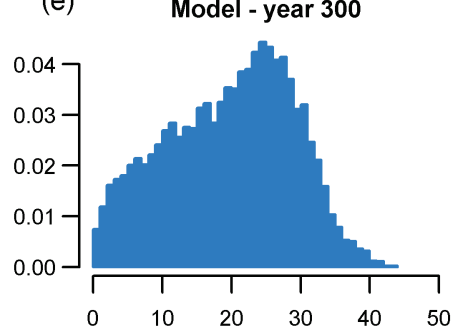

(h)
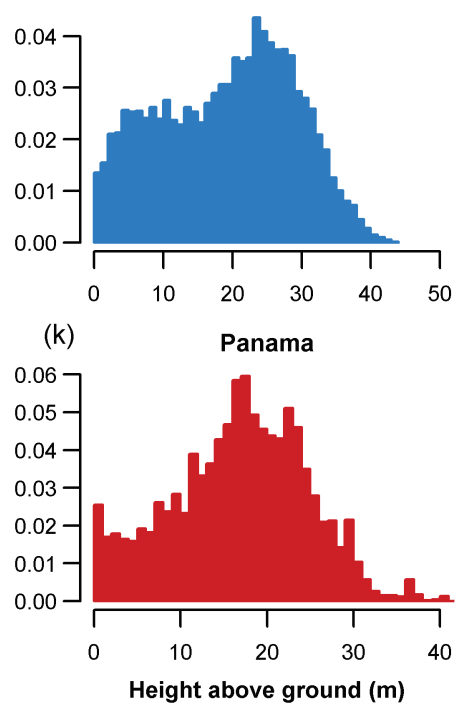

(c) Model - year 600

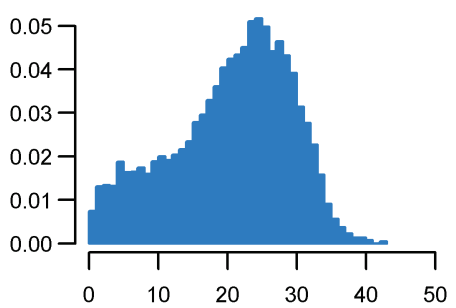

(f) Model - year 600

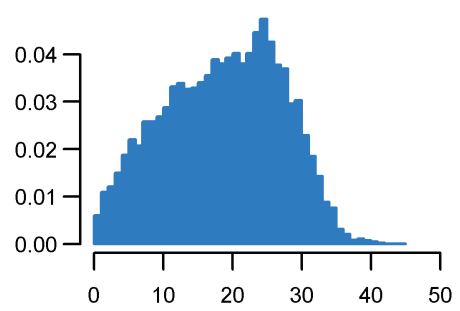

(i) Model - year 600

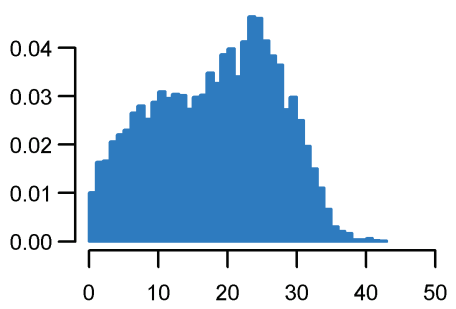

(I)

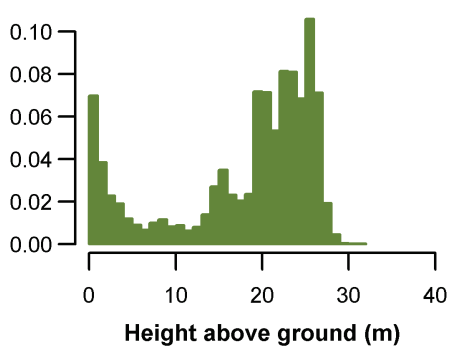

Figure D.6. Vertical distribution of epiphytes. The panels a-i show the vertical distribution of 3 different epiphyte assemblages simulated on forest stands representing typical lowland forest at the beginning of the simulations $(a, d, g)$, after 300 years $(b, e, h)$ and after 600 years $(\mathrm{c}, \mathrm{f}, \mathrm{i})$. The average vertical distribution of substrate area in theses forest stands is depicted in panel j. For comparisons: observed vertical distributions in Panama (k) and Ecuador (l). 
(a) Model - year 0

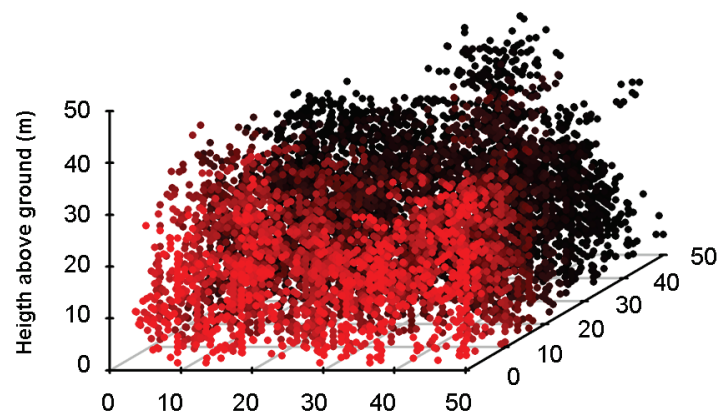

(c)

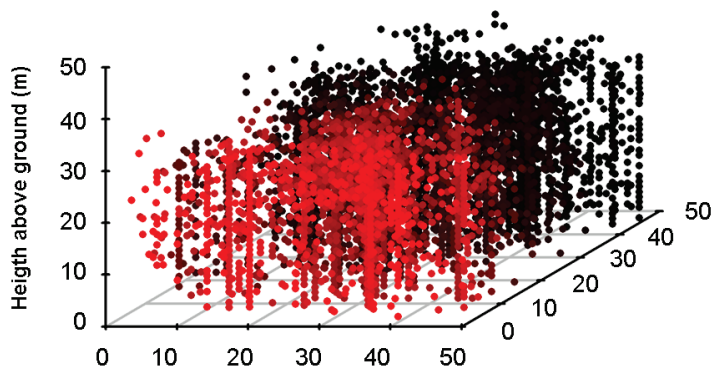

(e)

Panama

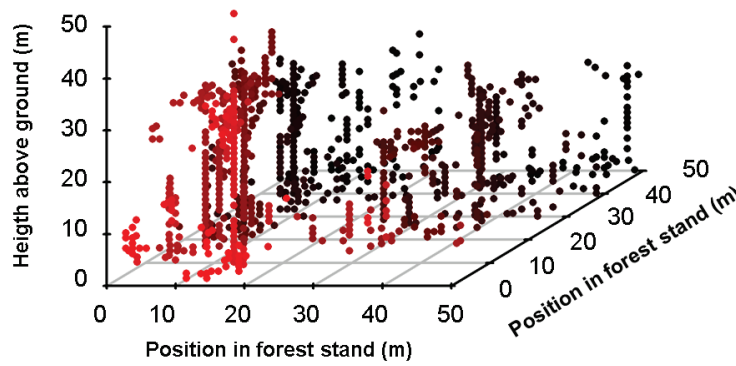

(b) Model - year 200

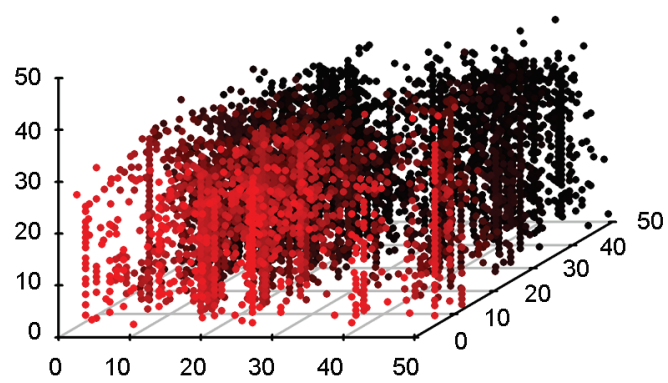

(d) Model - year 600

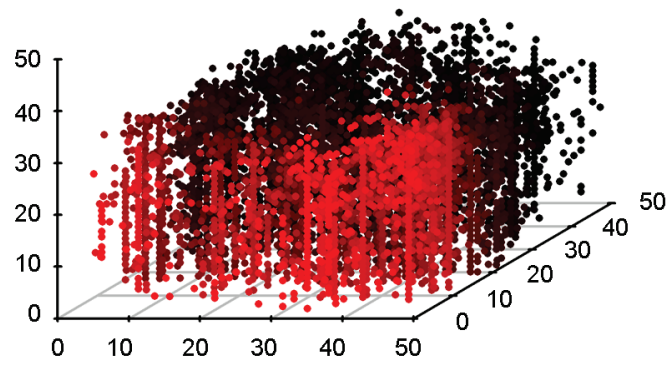

(f) Ecuador

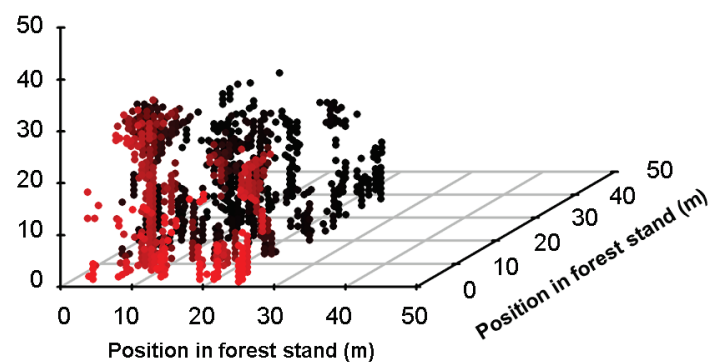

Figure D.7. 3D structure of epiphyte assemblages. Panels a-d show the simulated epiphyte assemblage in comparison to field data from Panama (e) and Ecuador (f). 
Appendix

Table D.1. Simulated mortality rates. The mean, minimum and maximum mortality rates for different causes of mortality of epiphytes on a typical lowland forest are shown here.

\begin{tabular}{lccc}
\hline Cause of mortality & Mean & Min & Max \\
\hline Fall attached to substrate & 3.63 & 1.25 & 14.92 \\
Fall off substrate & 2.44 & 1.34 & 7.22 \\
Changing environmental conditions & 0.45 & 0.00 & 5.57 \\
Space competition & 0.89 & 0.11 & 6.86 \\
Size-dependent mortality (metabolic theory) & 6.89 & 5.34 & 8.55 \\
\hline Total & 14.29 & 10.07 & 25.17 \\
\hline
\end{tabular}




\section{Acknowledgements}

First of all, I want to thank my supervisor, Prof. Dr. Holger Kreft, for his continuing encouragement and support. Thank you for your guidance and availability, for stimulating discussion and for creating such a friendly and motivating work environment. I really enjoyed working with you and learned a lot about how to do science.

I also want to thank my co-supervisor, Prof. Dr. Gerhard Zotz, for sharing his knowledge on epiphytes with me. He was always available and his critical comments greatly improved my thesis. Thank you for sharing your trait data and that you made it possible to use the canopy crane in Panama. That was a great experience.

My special thanks goes to Juliano Sarmento Cabral, who supervised and supported my work from the beginning to the end. He was my office mate for a long time and we had many fruitful discussions on epiphytes and modelling. Thank you for sharing you expert knowledge during field work, without you it would not have been possible.

I am thankful to Katrin Wagner for her substantial contributions to the trait paper. I also want to thank all my additional co-authors, Wolfgang Wanek, Glende Mendieta-Leiva and Eduardo Javier Sánchez Delgado, for their valuable suggestions and tireless feedback.

I thank Prof. Winfried Kurth for introducing me to functional-structural plant models and for developing GroIMP. I also want to thank Yongzhi Ong for helping me programming my forest model.

Many thanks to all my lab mates in the Macroecology, Biodiversity and Conservation Biogeography Group for lively lab-meeting and fruitful discussions. I always enjoyed coming to office.

I thank the DFG (German Research Foundation) for funding my position at the University of Göttingen in the framework of the German Excellence Initiative.

Finally, I am most grateful to my family and friends for their many ways of support. 



\section{Erklärung}

Hiermit versichere ich, dass ich die vorliegende Dissertation selbständig angefertigt und die benutzten Quellen und Hilfsmittel vollständig angegeben habe. Die Dissertation wurde an keiner anderen Hochschule eingereicht und wurde, abgesehen von den unter "Author contributions“ angegebenen Teilpublikationen, noch nicht veröffentlicht. Außerdem erkläre ich, dass ich bisher noch keinen Promotionsversuch unternommen habe.

Göttingen, im April 2016

Gunnar Petter 